\title{
Evaluating the Role of Molecular Heredity on the Optical and Electronic Properties of Cross-Conjugated Benzo[1,2-d:4,5-d']bisoxazoles
}

Authors: David L. Wheeler, ${ }^{a}$ Alex V. Diodati, ${ }^{\text {b,c }}$ Aimée L. Tomlinson, ${ }^{\mathrm{b}}$ and Malika Jeffries-EL*a, d

a) Department of Chemistry, Boston University, 590 Commonwealth Ave, Boston, MA 02215, USA.

b) Department of Chemistry and Biochemistry, University of North Georgia, 82 College Circle, Dahlonega GA, 30041, USA.

c) Department of Chemistry, University of Florida, Leigh Hall, Gainesville, FL 32603, USA.

d) Division of Materials Science and Engineering, Boston University, 15 St. Mary Street, Boston MA02215, USA.

\section{Supplemental Information}




\section{Table of Contents}

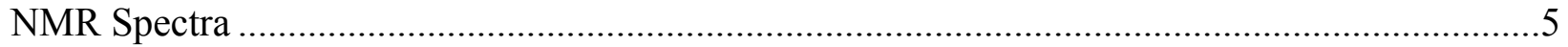

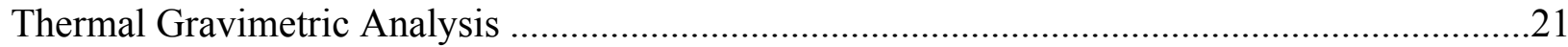

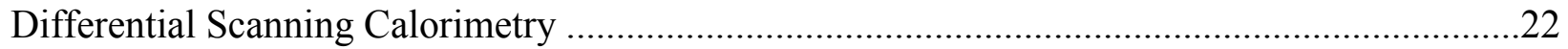

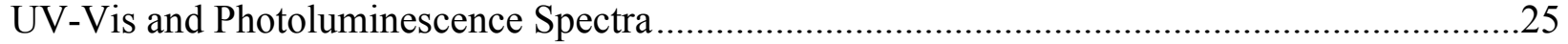

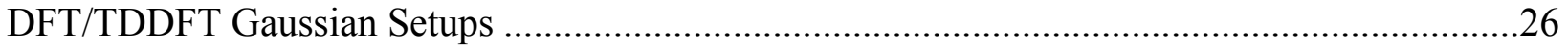

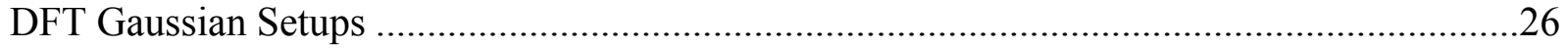

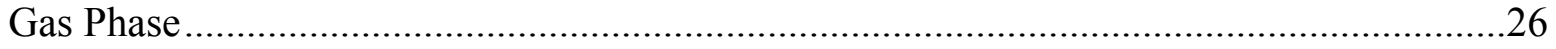

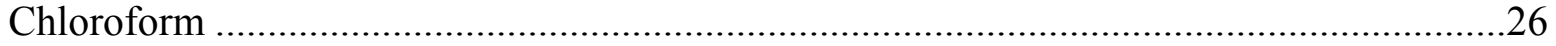

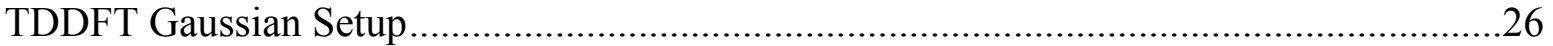

Number of Imaginary Frequencies \& Internal Energies for all in Gas and Chloroform Phases ...27

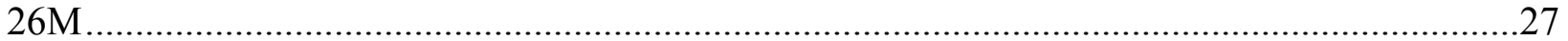

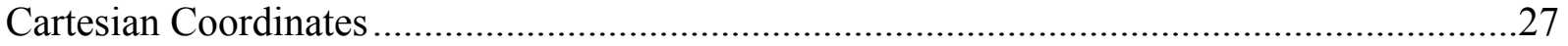

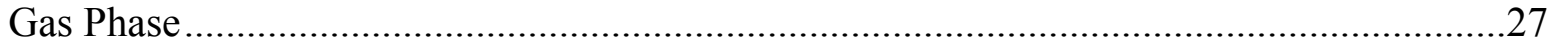

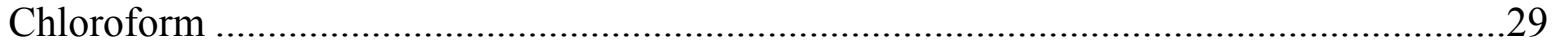

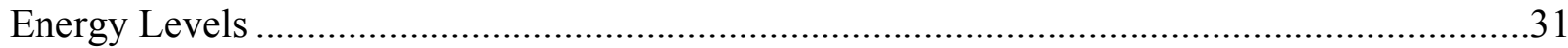

Gas Phase

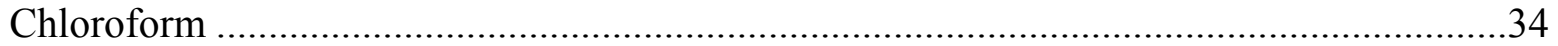

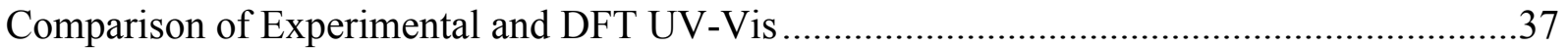

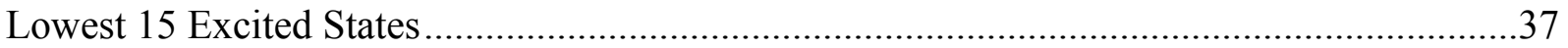

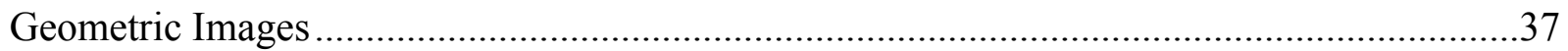

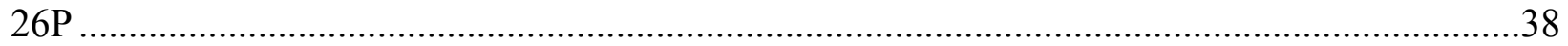

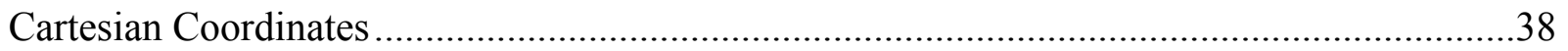

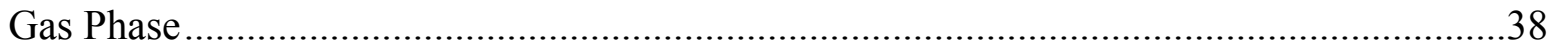

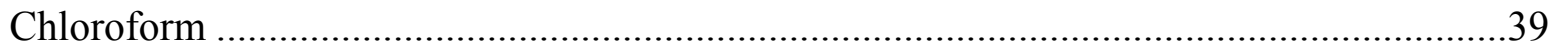

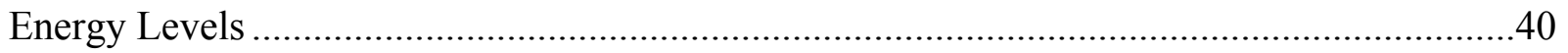

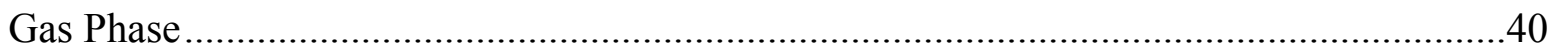

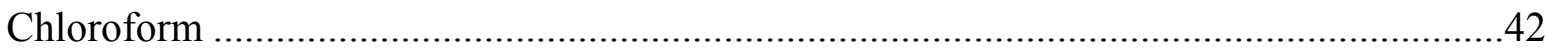

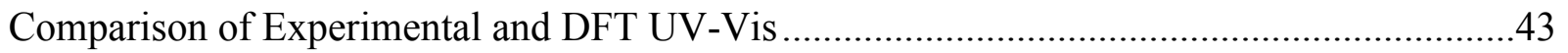

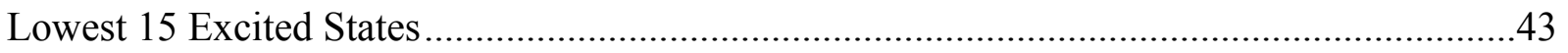

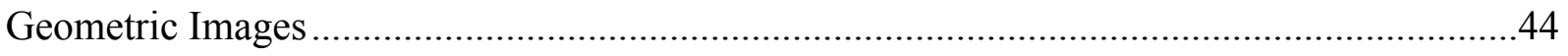

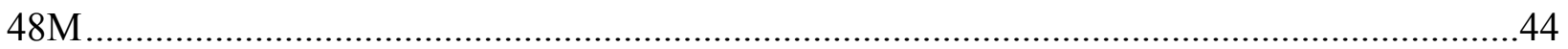

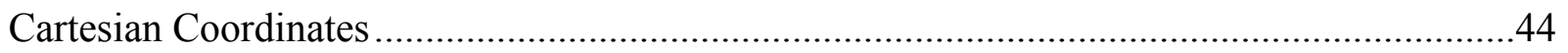

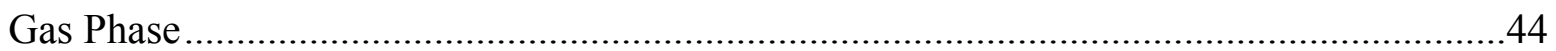




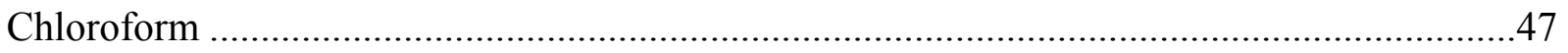

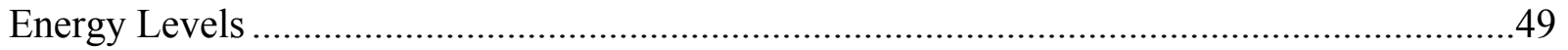

Gas Phase

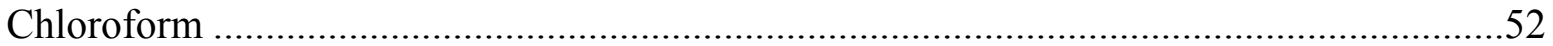

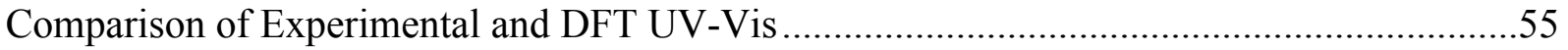

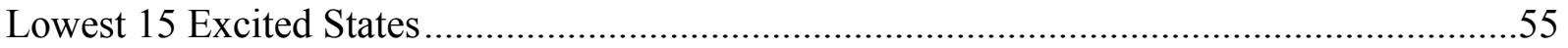

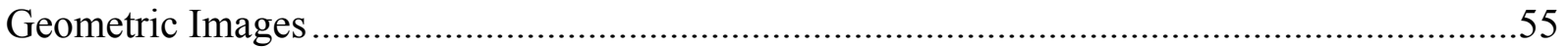

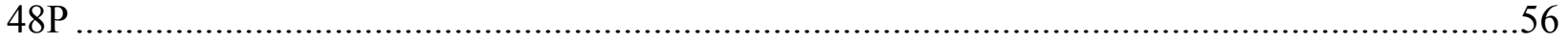

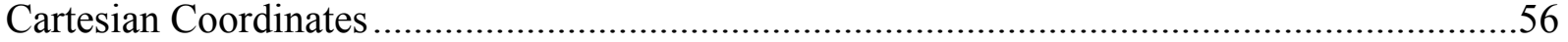

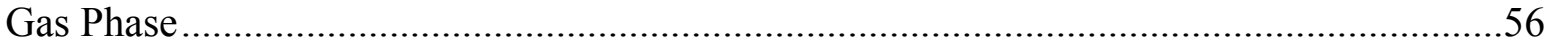

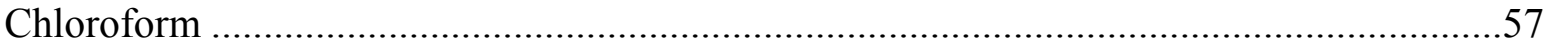

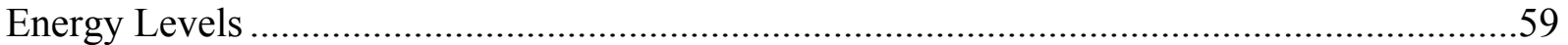

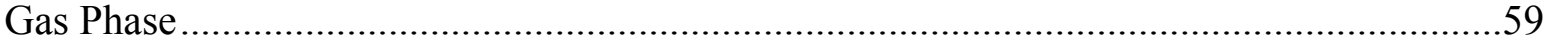

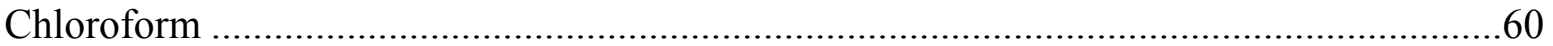

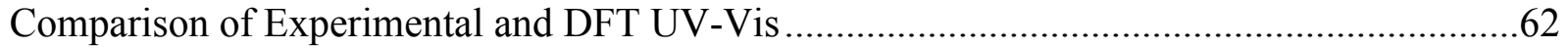

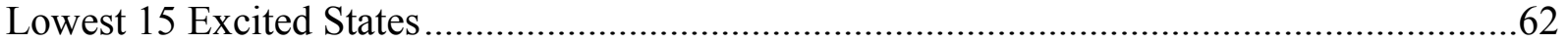

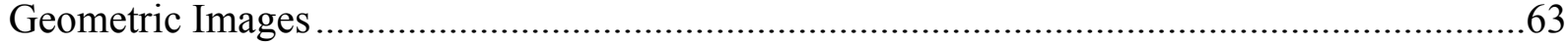

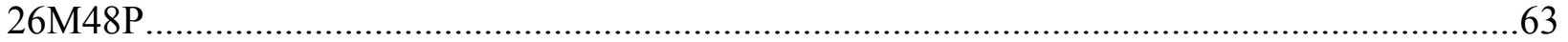

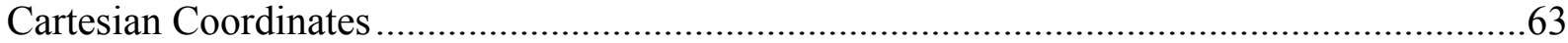

Gas Phase

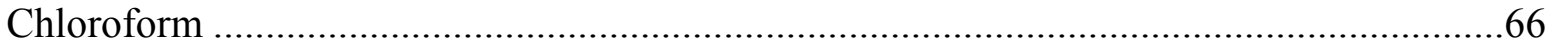

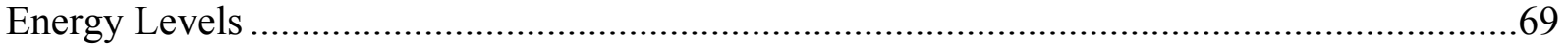

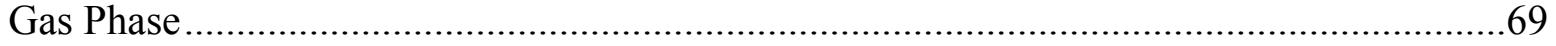

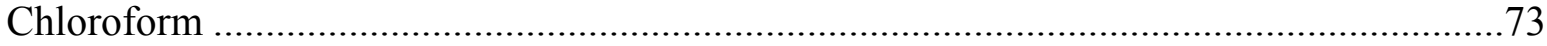

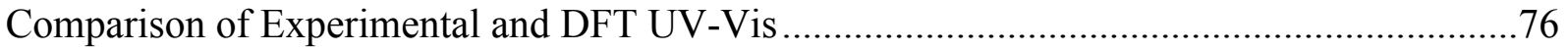

Lowest 15 Excited States...............................................................................................

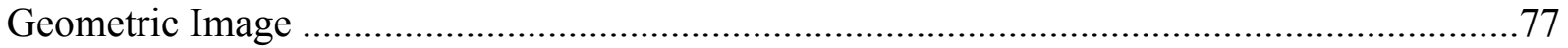

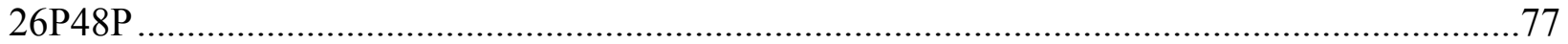

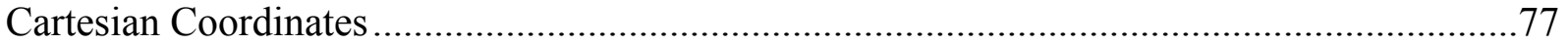

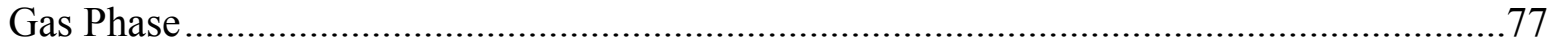

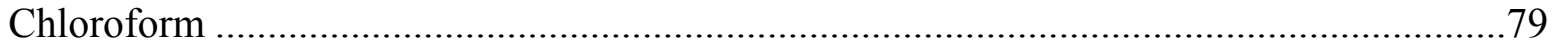

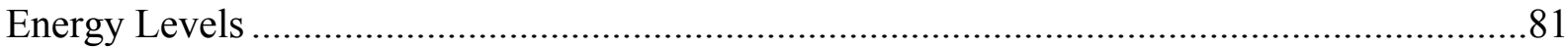

Gas Phase

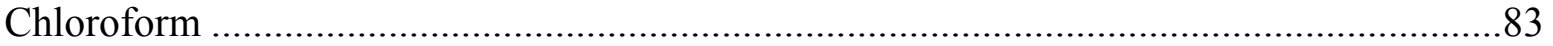




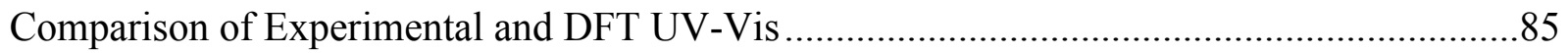

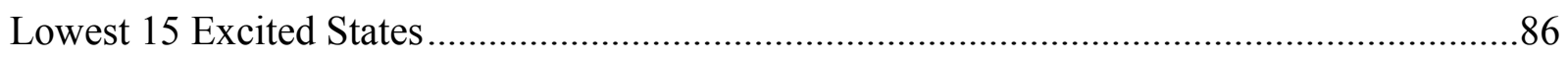

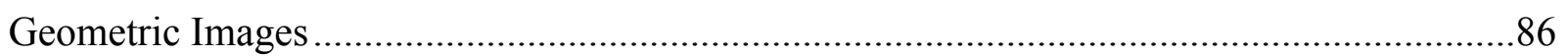

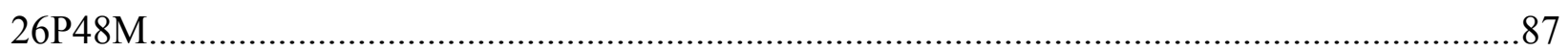

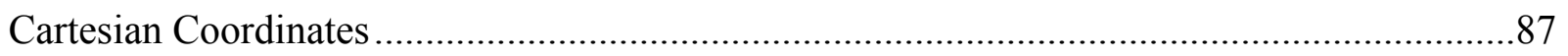

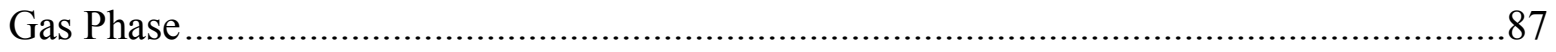

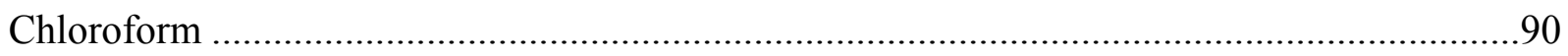

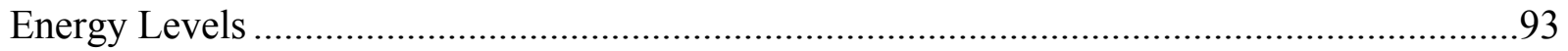

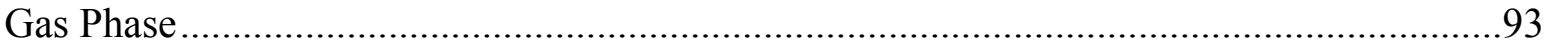

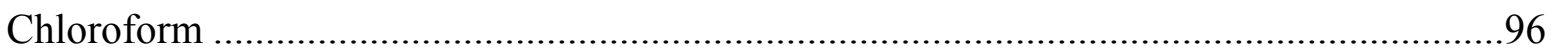

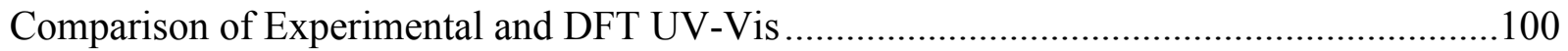

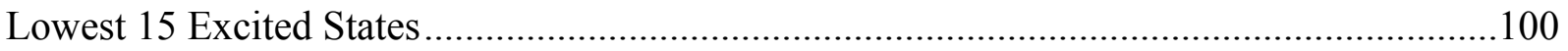

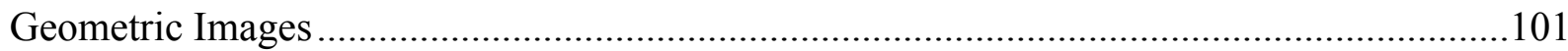

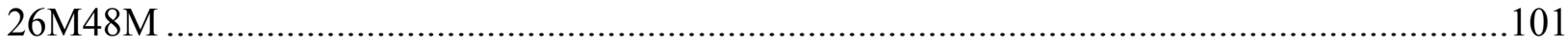

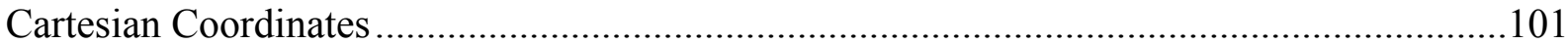

Gas Phase

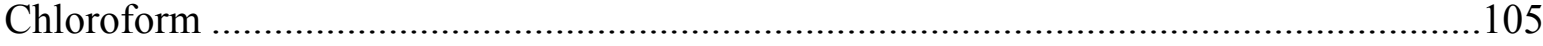

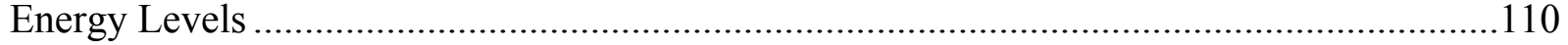

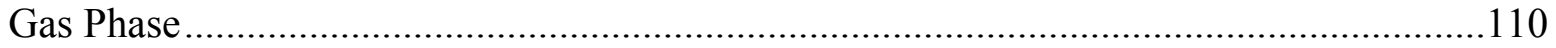

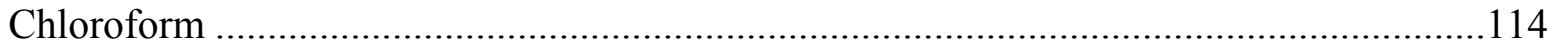

Comparison of Experimental and DFT UV-Vis.............................................................119

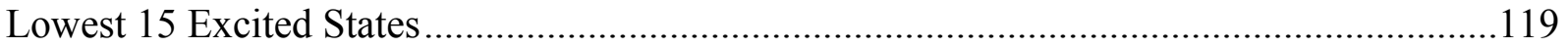

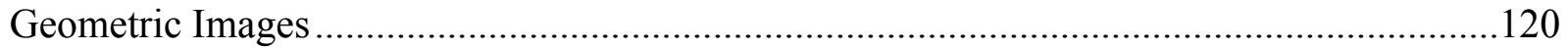

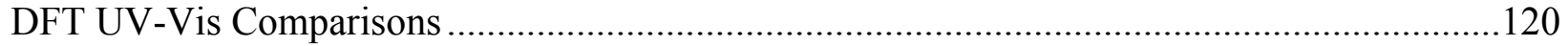

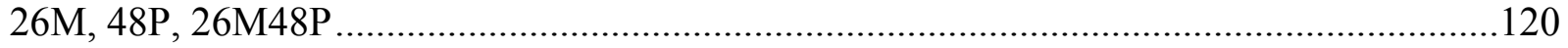

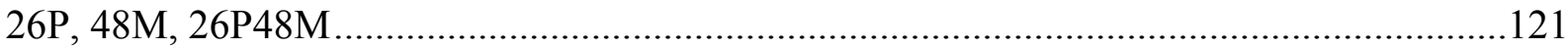

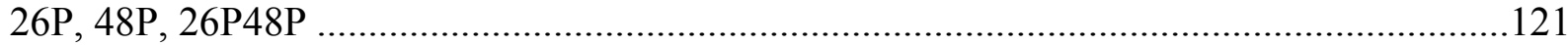

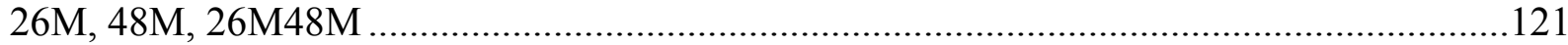

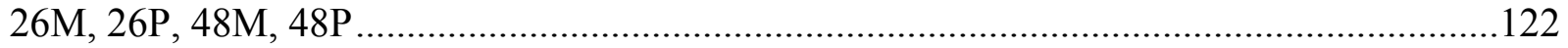

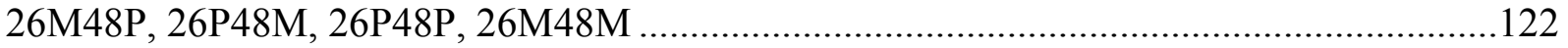

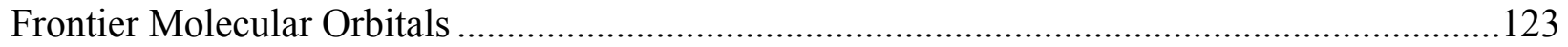




\section{NMR Spectra}
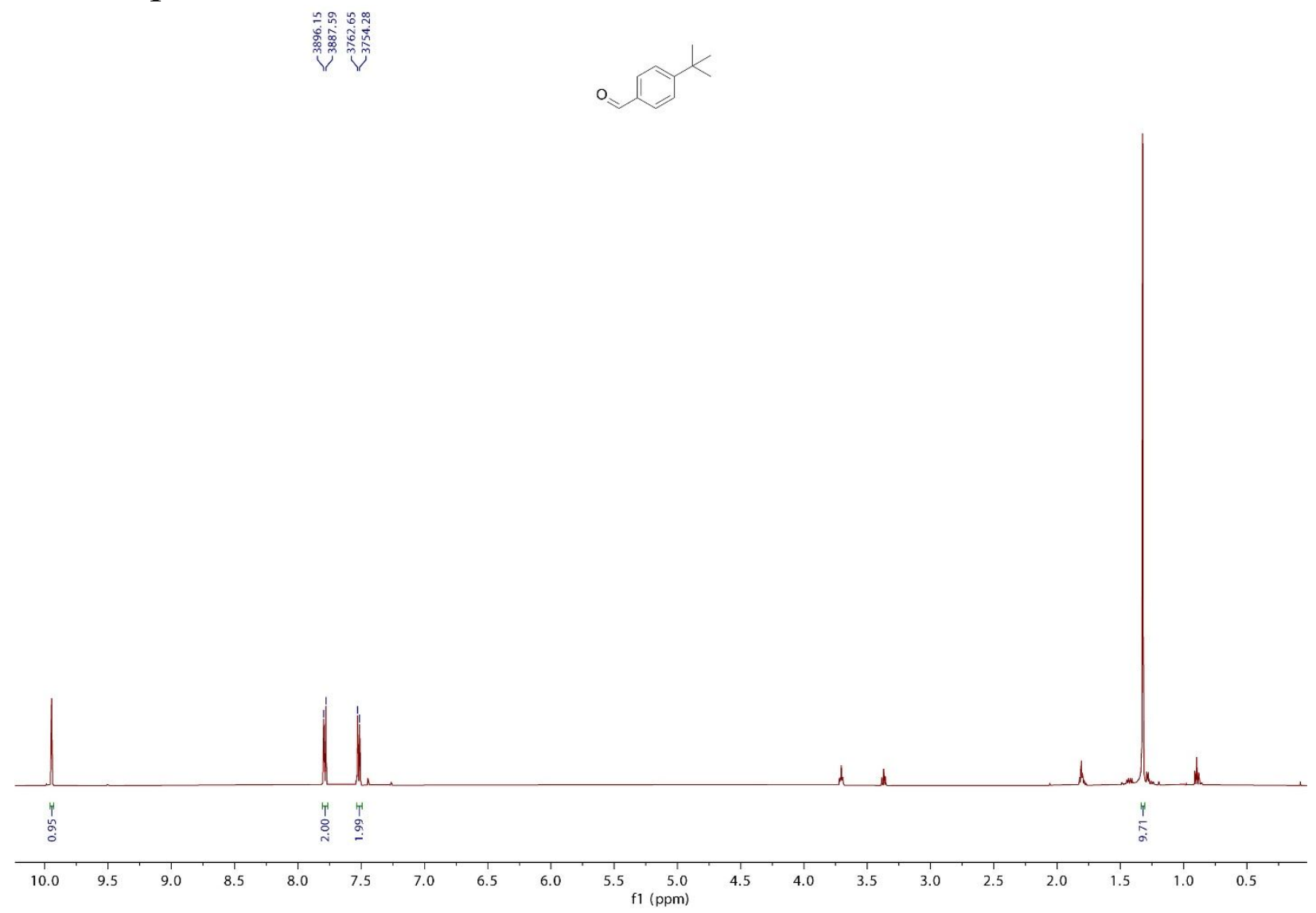

Figure S1. ${ }^{1} \mathrm{H}$ NMR of 1 


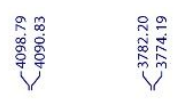
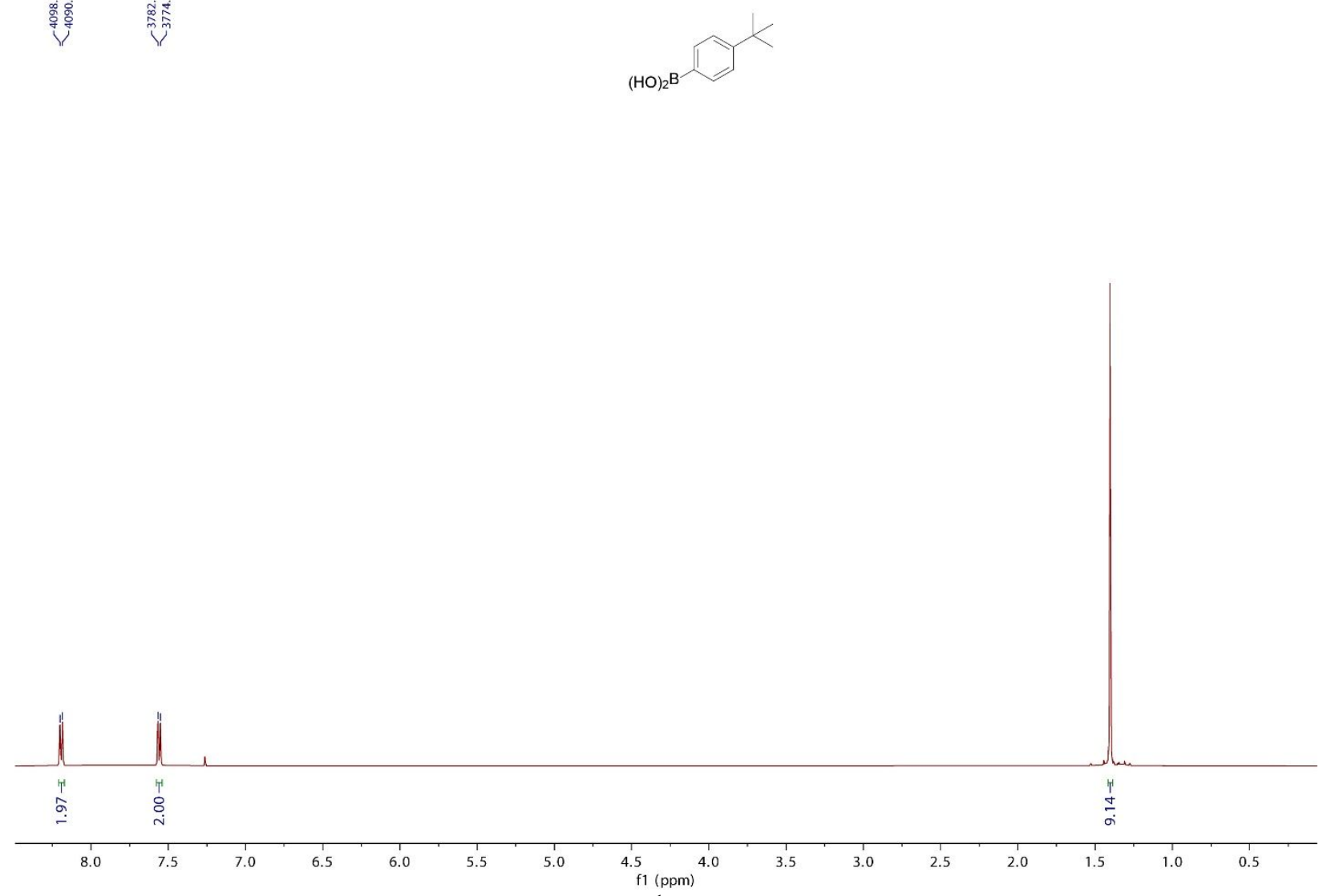

Figure S2. ${ }^{1} \mathrm{H}$ NMR of 2 


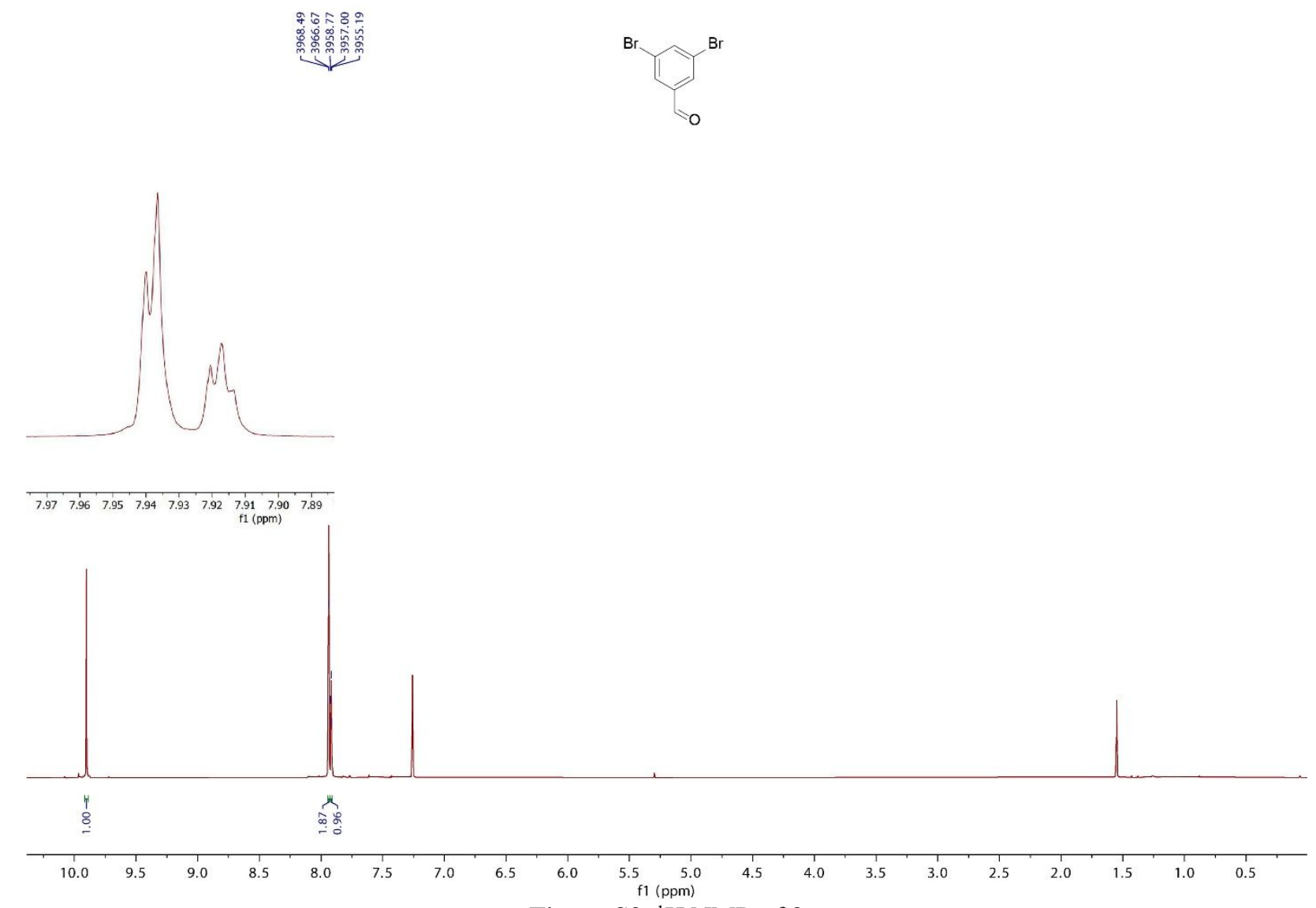

Figure S3. ${ }^{1} \mathrm{H}$ NMR of 3 


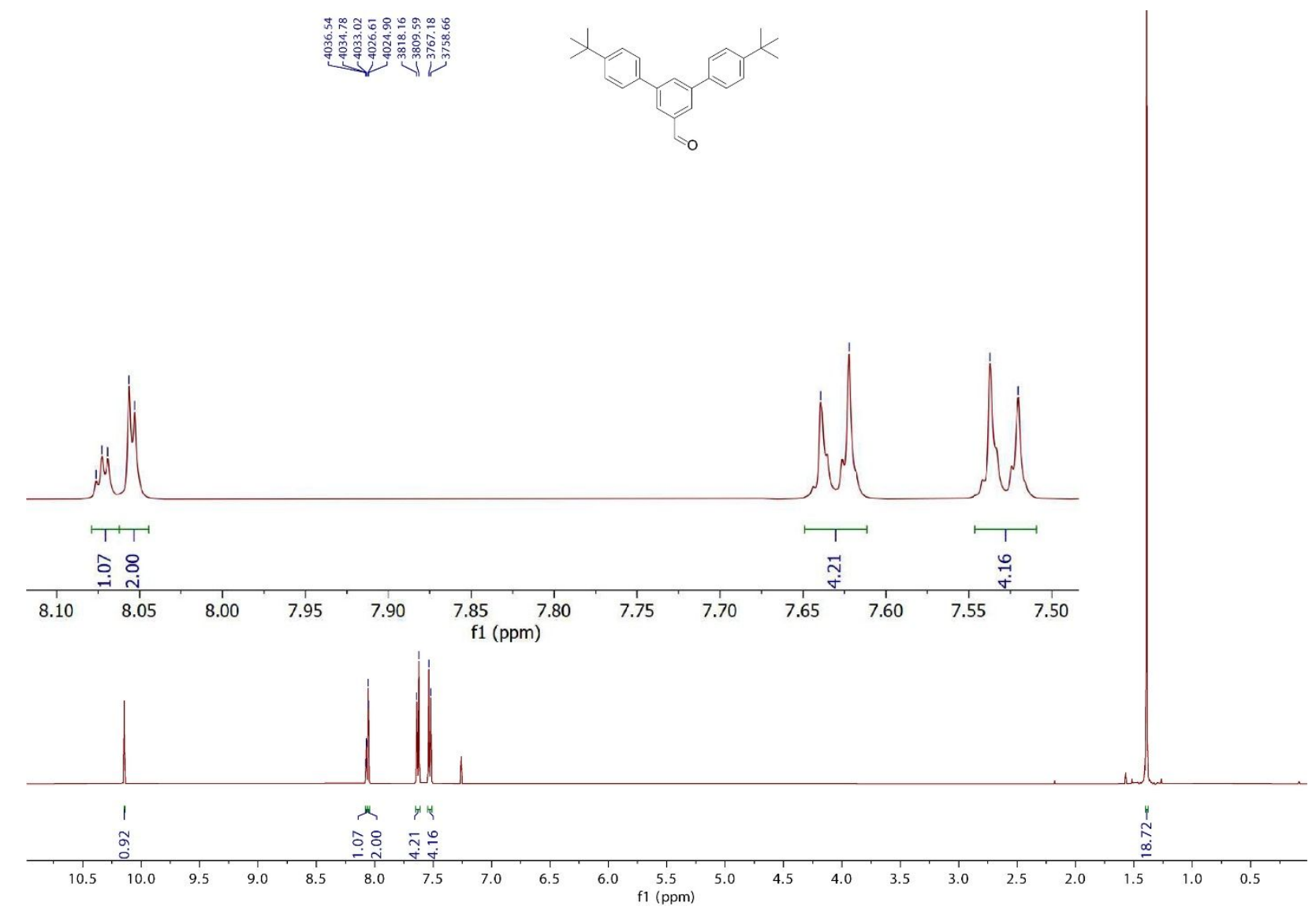

Figure S4. ${ }^{1} \mathrm{H}$ NMR of 4 

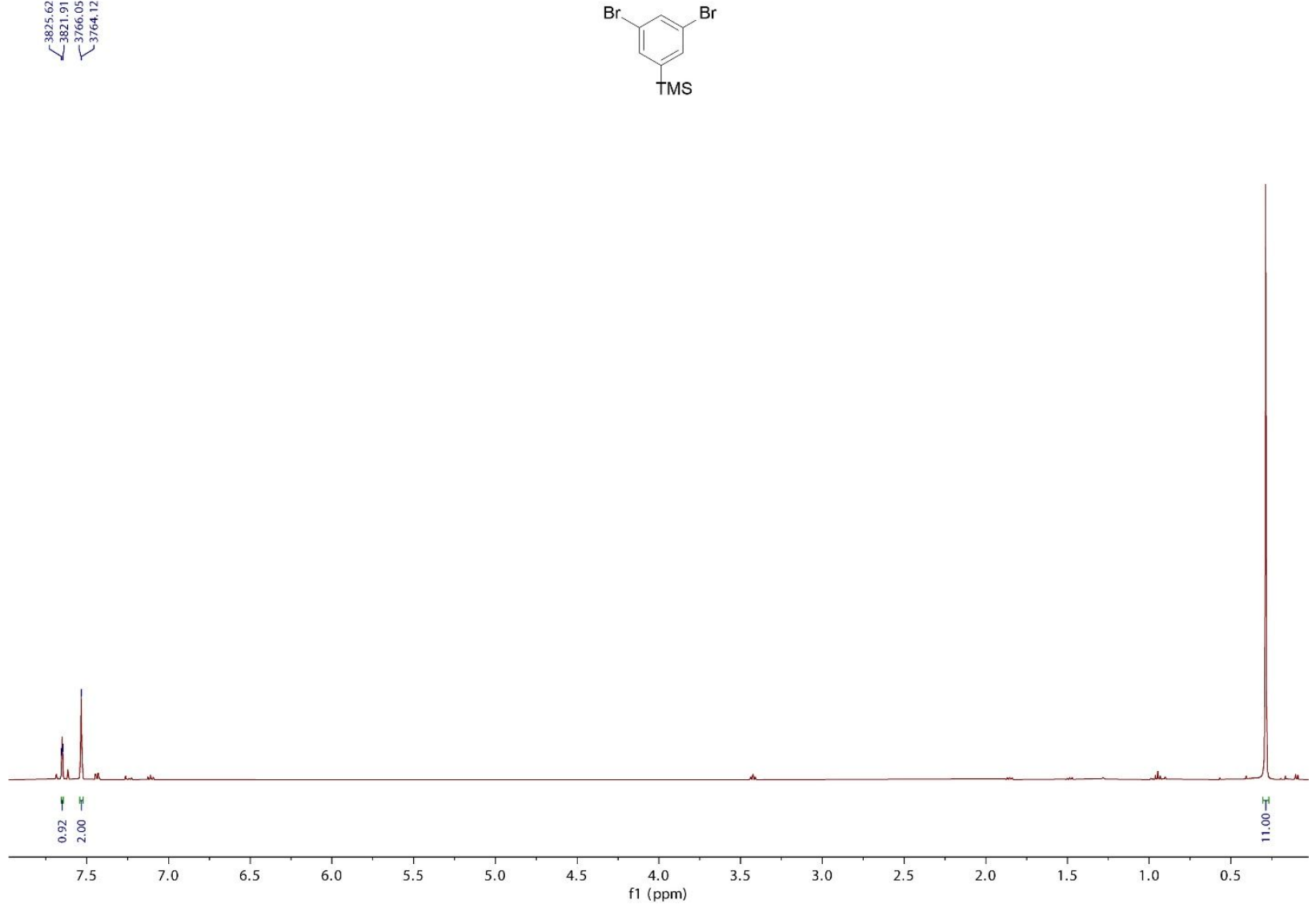

Figure S5. ${ }^{1} \mathrm{H}$ NMR of 5 


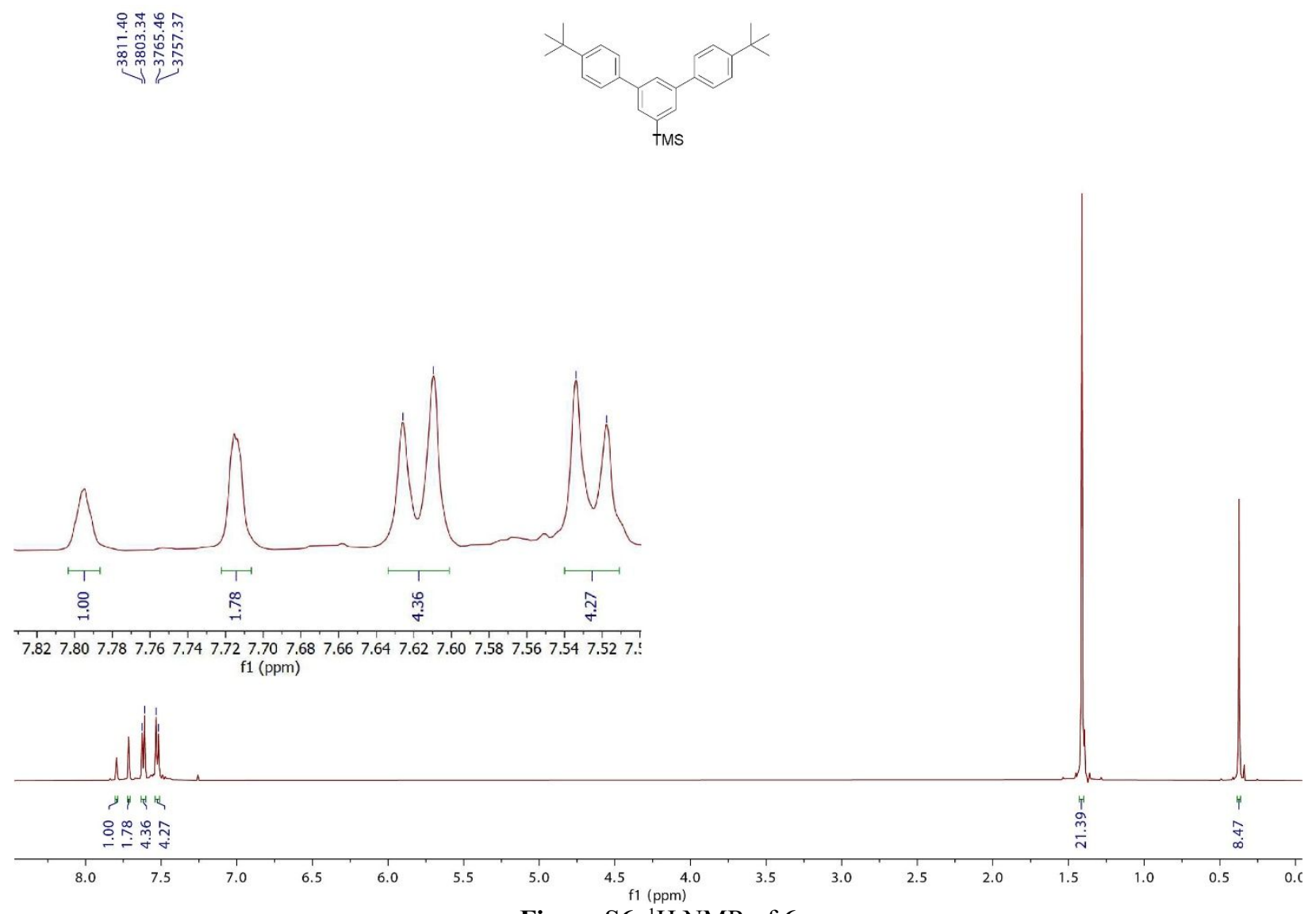

Figure S6. ${ }^{1} \mathrm{H}$ NMR of 6 


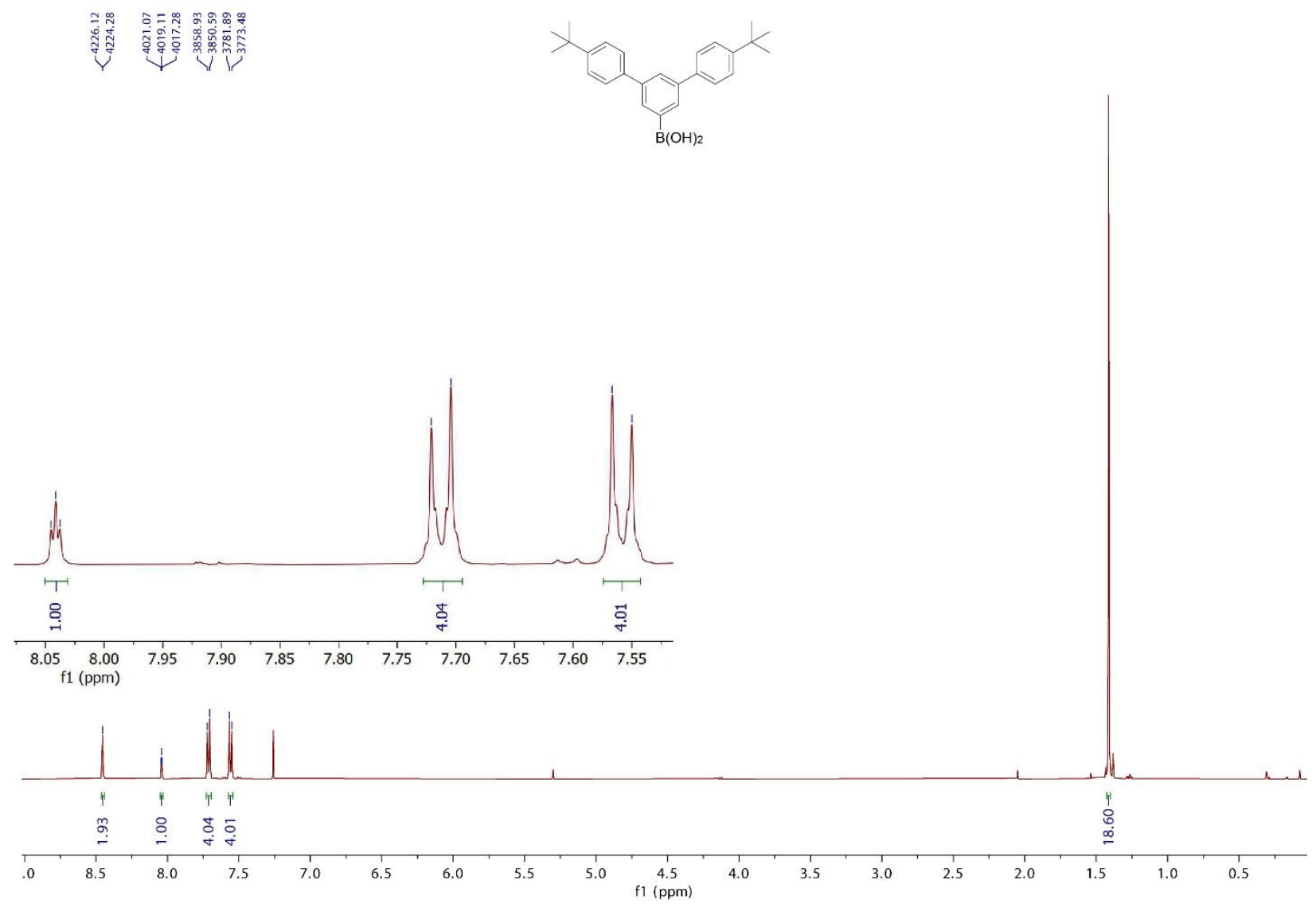

Figure S7. ${ }^{1} \mathrm{H}$ NMR of 7 


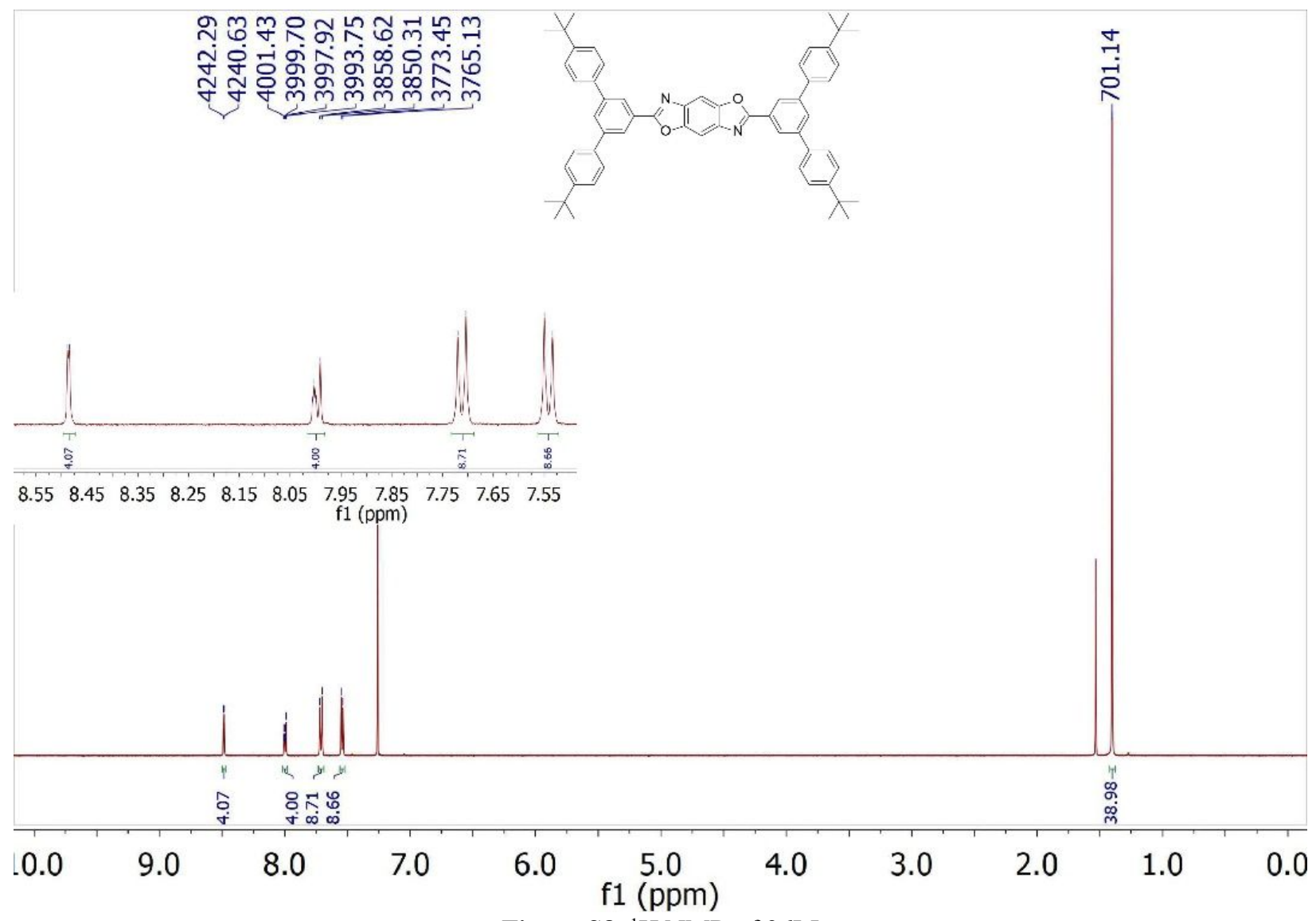

Figure S8. ${ }^{1} \mathrm{H}$ NMR of $\mathbf{2 6 M}$ 


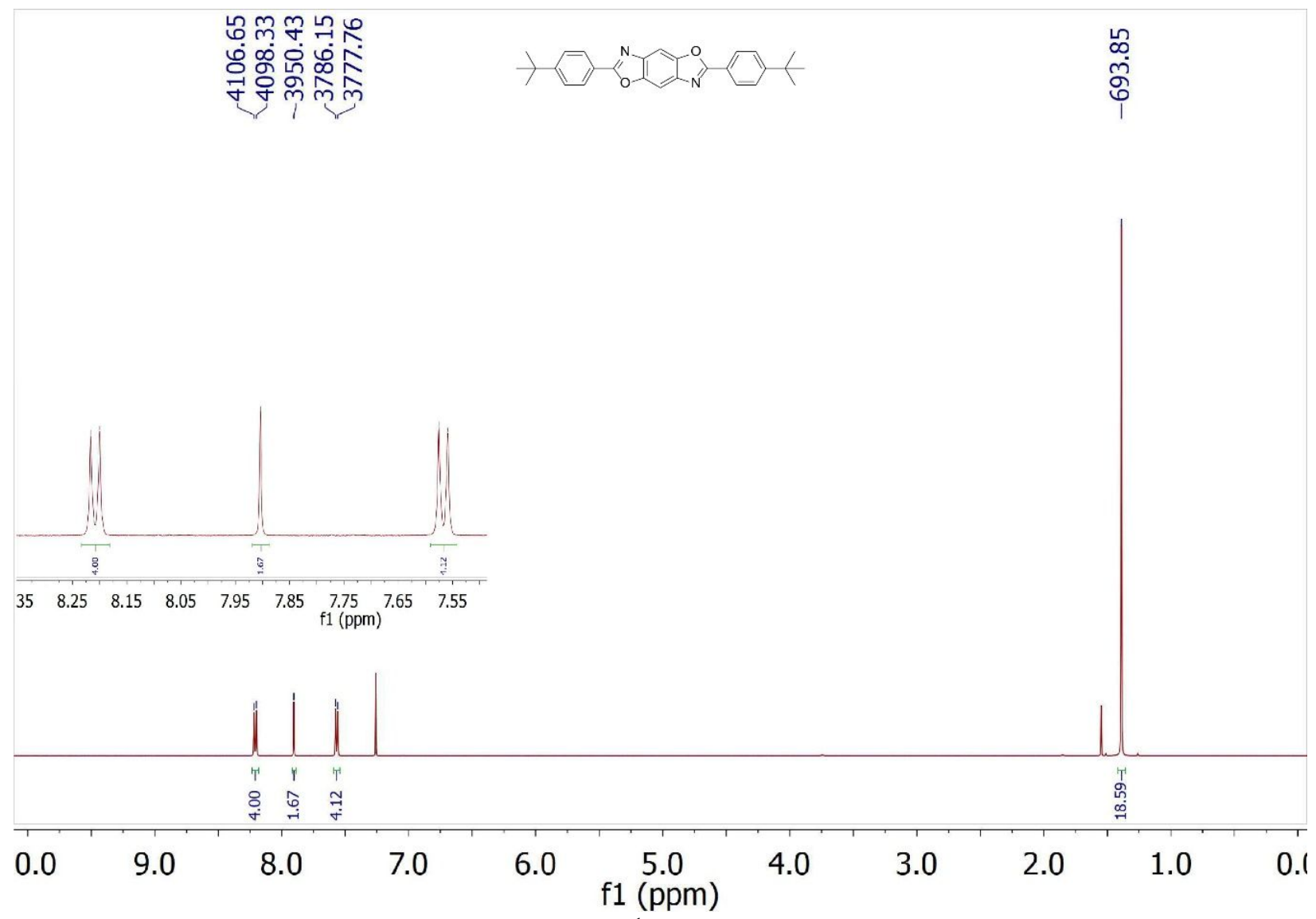

Figure S9. ${ }^{1} \mathrm{H}$ NMR of 26P 


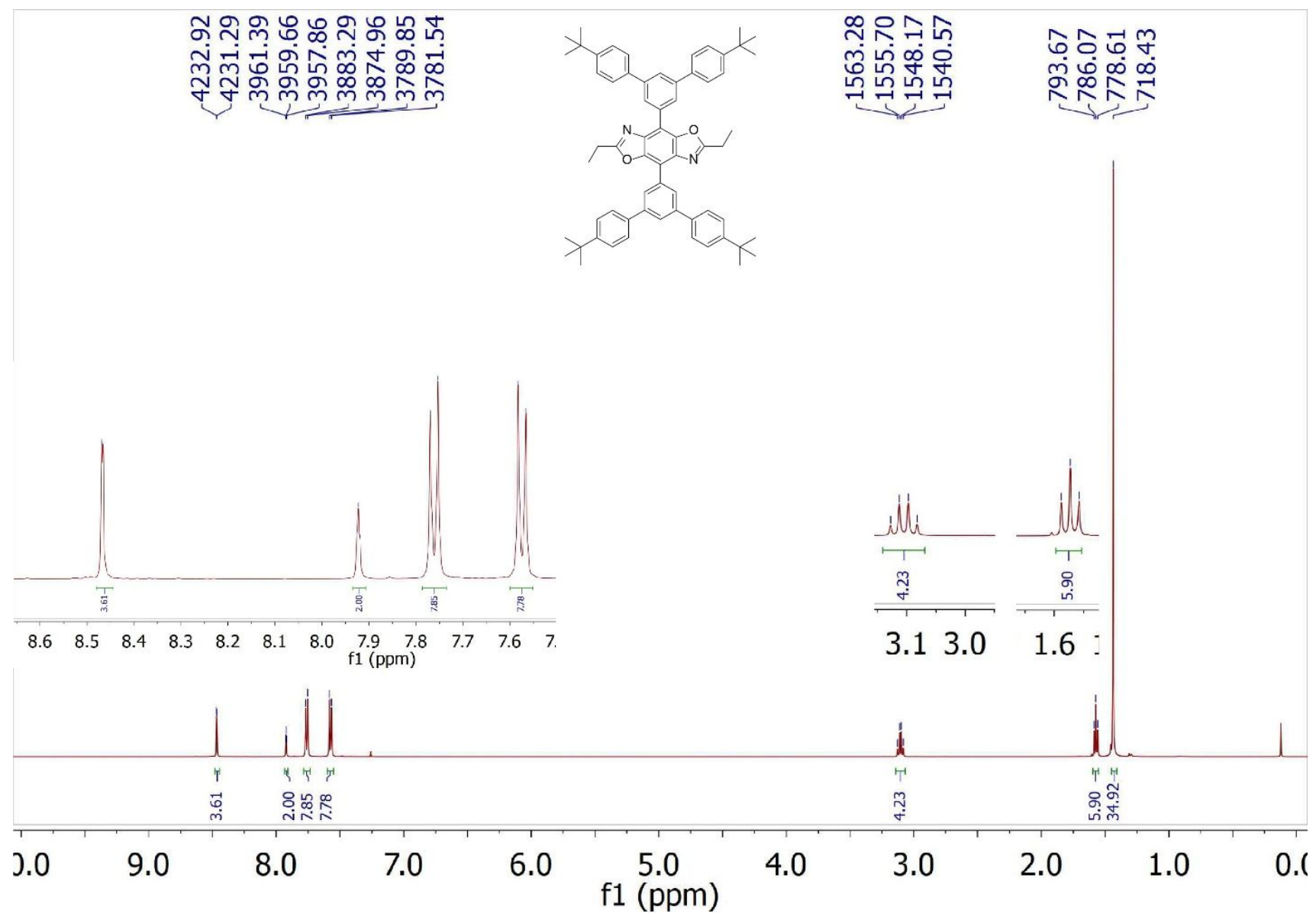

Figure S10. ${ }^{1} \mathrm{H}$ NMR of $\mathbf{4 8 M}$ 


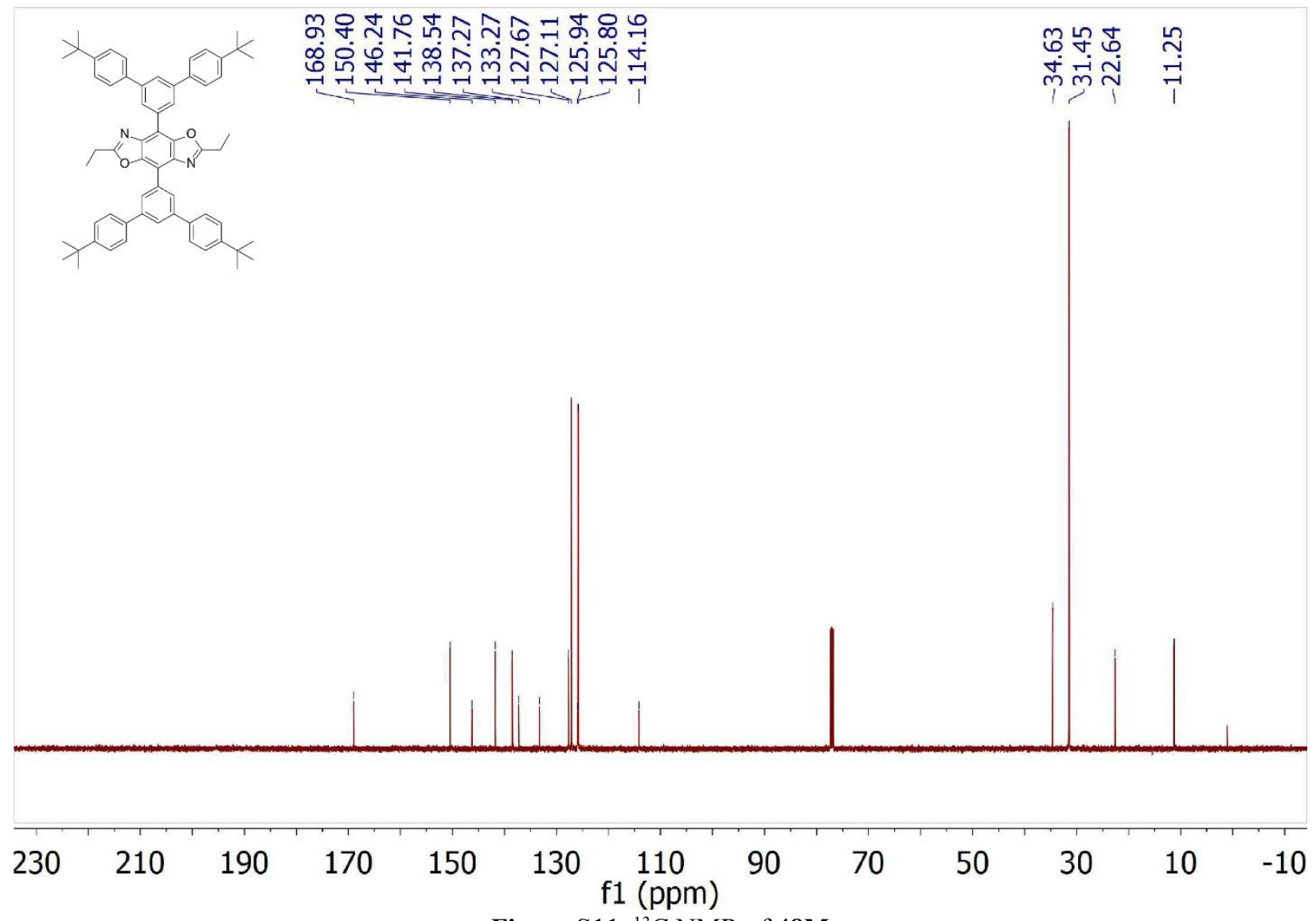

Figure S11. ${ }^{13} \mathrm{C}$ NMR of $48 \mathrm{M}$ 


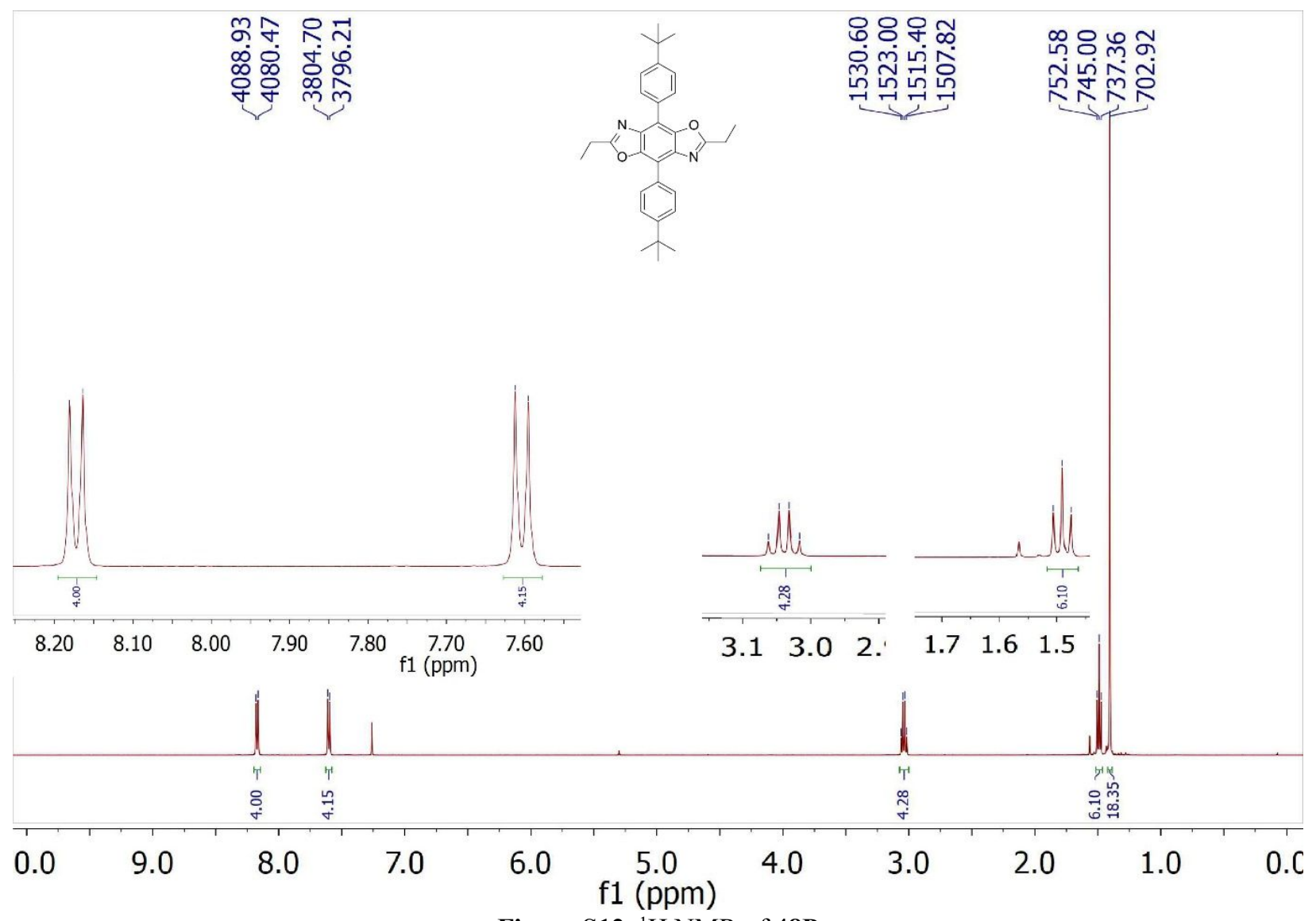

Figure S12. ${ }^{1} \mathrm{H}$ NMR of $48 \mathrm{P}$ 


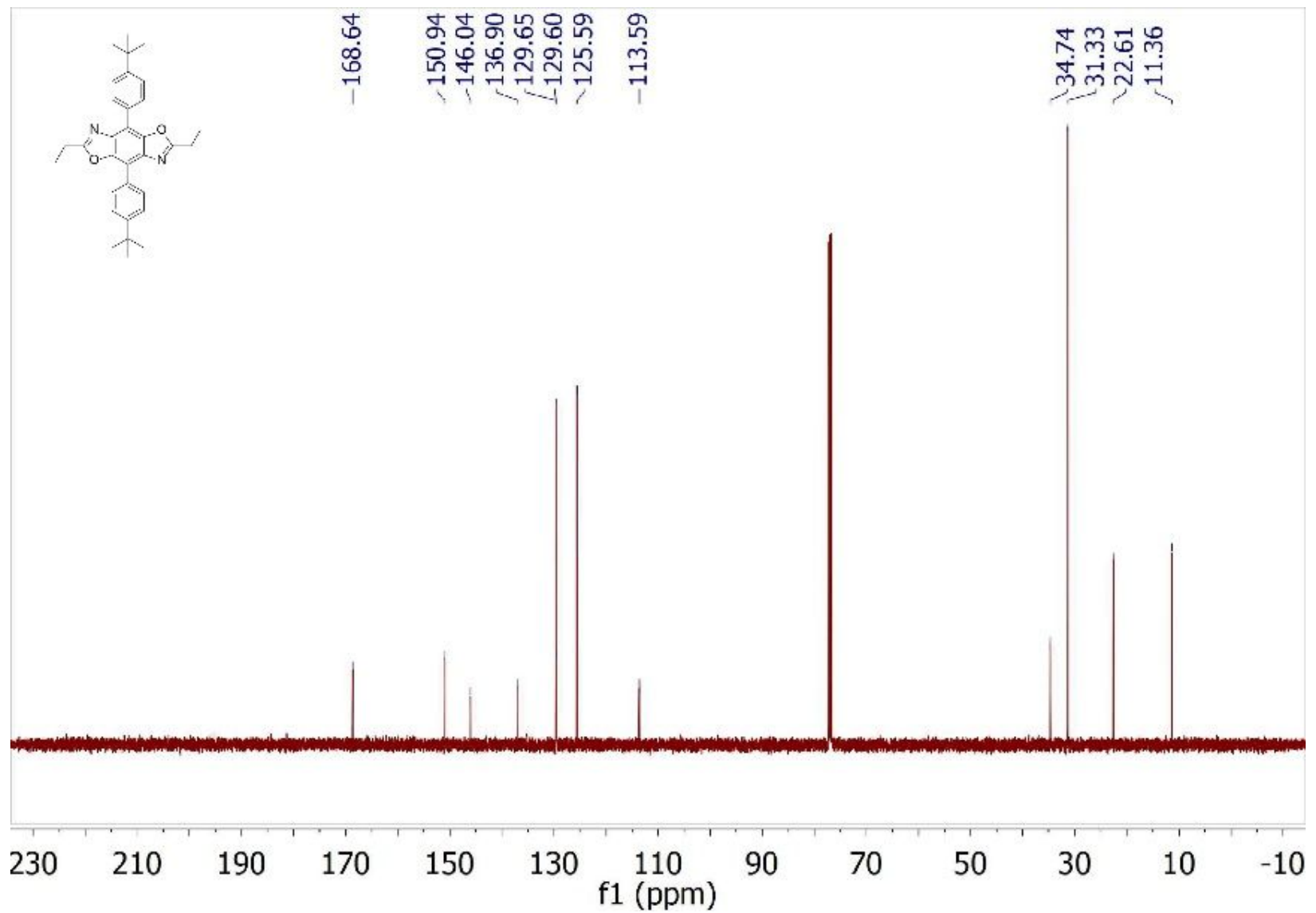

Figure S13. ${ }^{13} \mathrm{C}$ NMR of $48 \mathrm{P}$ 


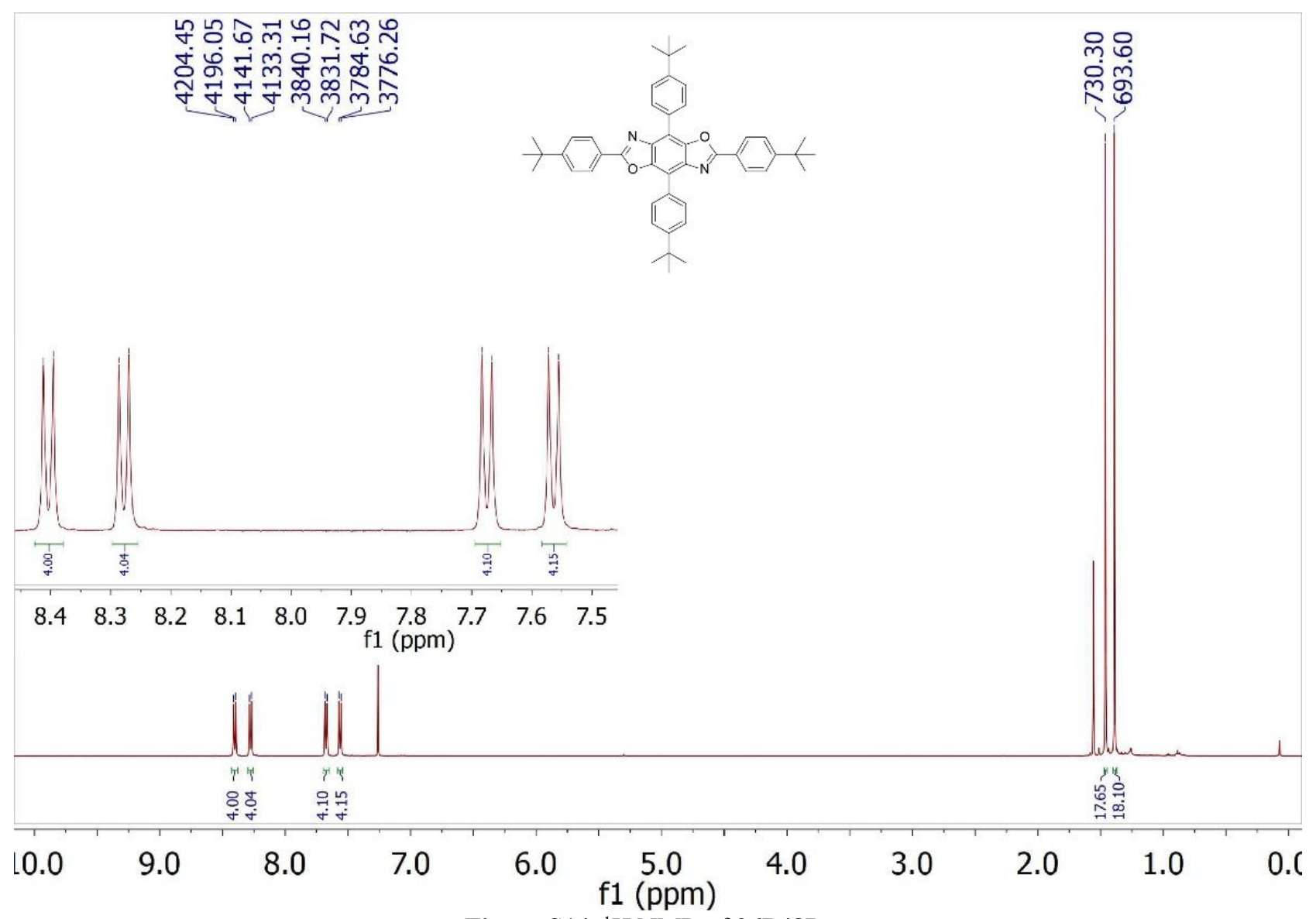

Figure S14. ${ }^{1} \mathrm{H}$ NMR of 26P48P 


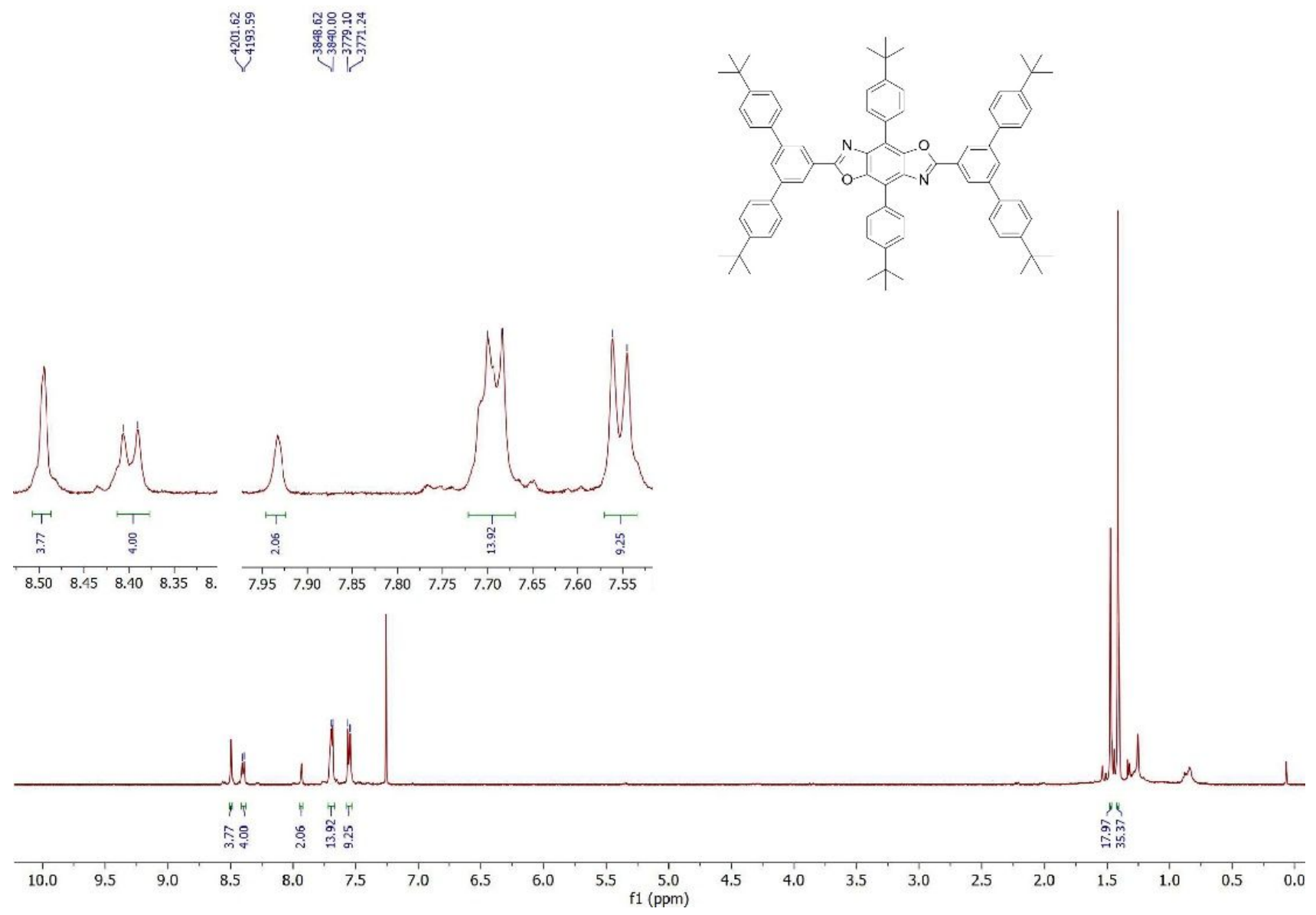

Figure S15. ${ }^{1} \mathrm{H}$ NMR of 26M48P 


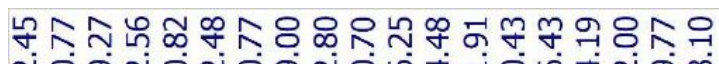

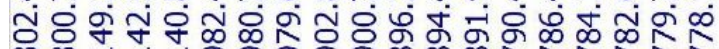
भु户

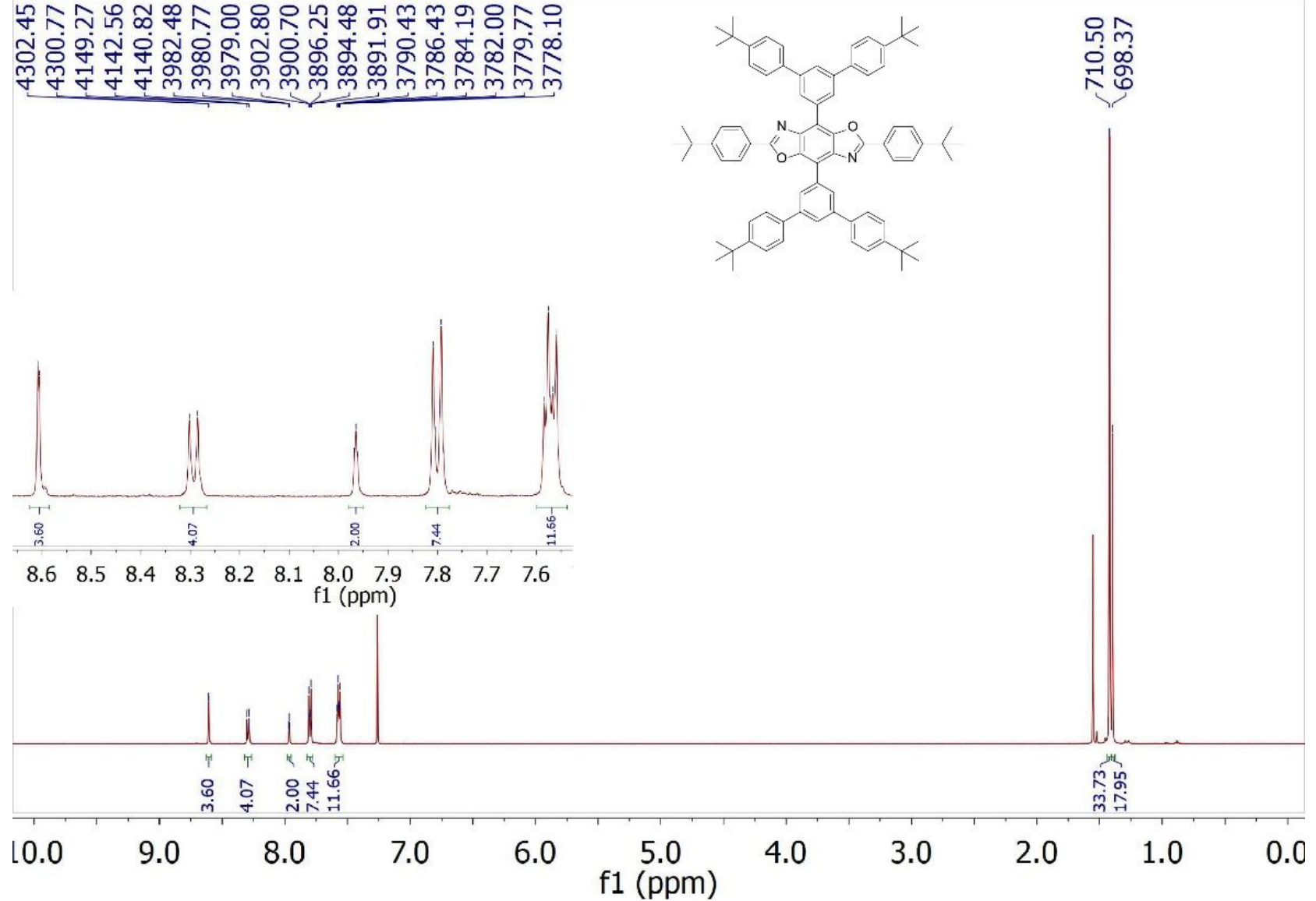

유

Figure S16. ${ }^{1} \mathrm{H}$ NMR of 26P48M 


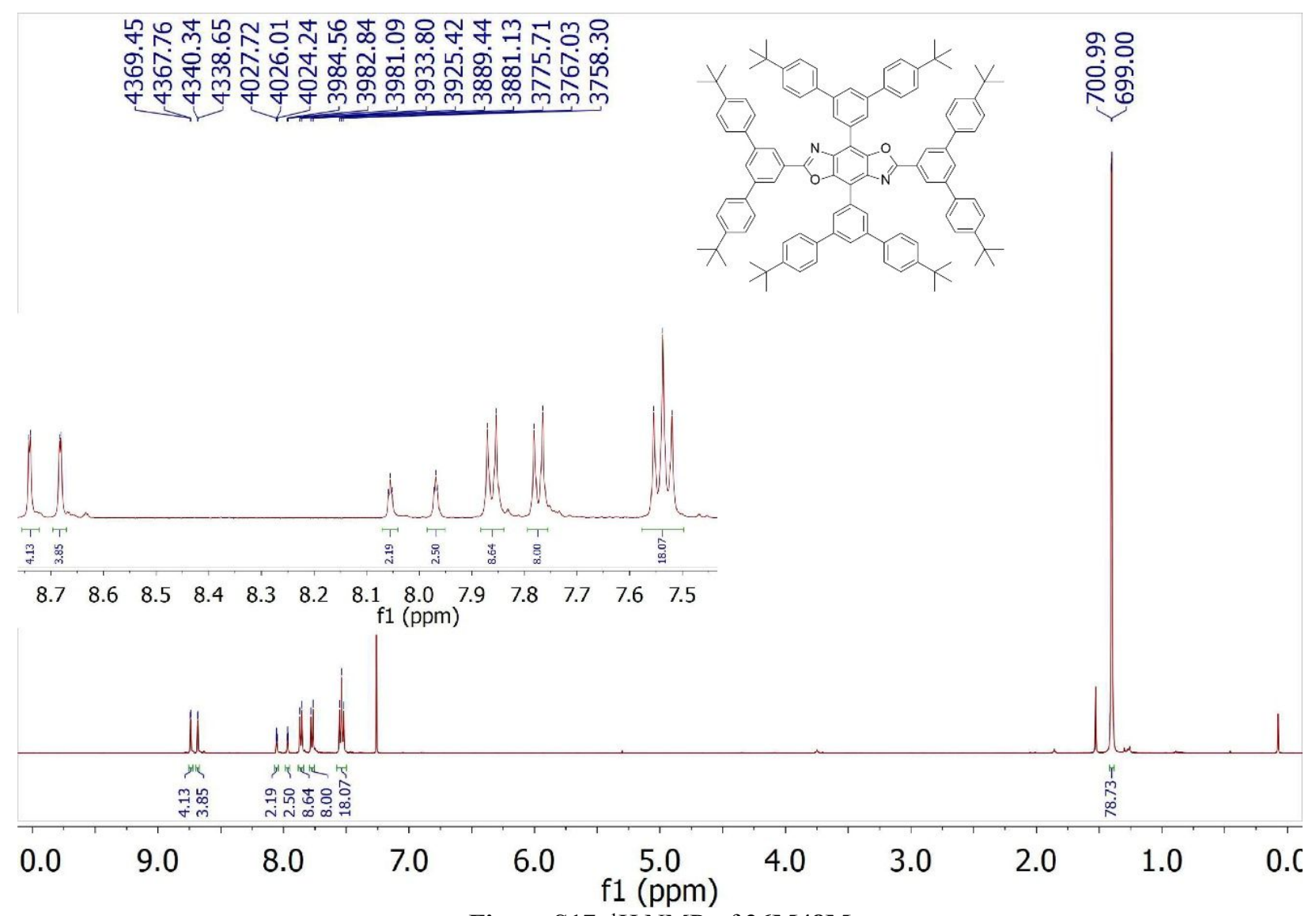

Figure S17. ${ }^{1} \mathrm{H}$ NMR of 26M48M

\section{Thermal Gravimetric Analysis}

All thermal gravimetric analysis (TGA) measurements were performed as follows under a nitrogen atmosphere: To begin, each sample was heated at a rate of $10^{\circ} \mathrm{C} / \mathrm{min}$ to $110^{\circ} \mathrm{C}$ and held at this temperature for 20 minutes. Next, each sample was heated at a rate of $10^{\circ} \mathrm{C} / \mathrm{min}$ to $600{ }^{\circ} \mathrm{C}$ and held at this temperature for a period of $10 \mathrm{~min}$. The data collected after the 20 -minute period is summarized in Figure SI16 and Table SI1.

Table S1. Summary of 5\% decomposition temperature for the BBOs.

\begin{tabular}{|c|c|c|c|c|c|c|c|c|}
\hline Compound & $\mathbf{2 6 M}$ & $\mathbf{2 6 P}$ & $\mathbf{4 8 M}$ & $\mathbf{4 8 P}$ & $\mathbf{2 6 P 4 8 P}$ & $\mathbf{2 6 M 4 8 P}$ & $\mathbf{2 6 P 4 8 M}$ & $\mathbf{2 6 M 4 8 M}$ \\
\hline $\boldsymbol{T g}\left({ }^{\circ} \boldsymbol{C}\right)$ & 468 & 280 & 384 & 272 & 334 & 432 & 432 & 310 \\
\hline
\end{tabular}



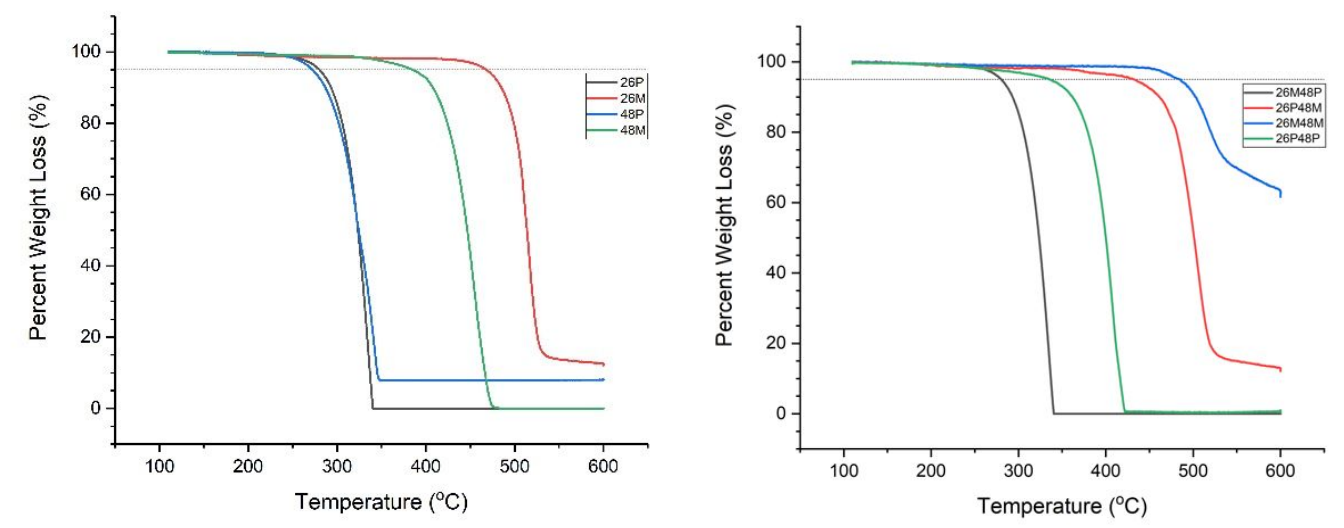

Figure S18. TGA plots of the eight target compound. The horizontal asymptote indicates $5 \%$ weight loss of each sample.

\section{Differential Scanning Calorimetry}

All differential scanning calorimetry (DSC) measurements were performed in the

following manner under a nitrogen atmosphere. All data shown represents the second heating/cooling cycle. The red curve indicates the heating cycle of each compound while the blue corresponds to the cooling curve. 

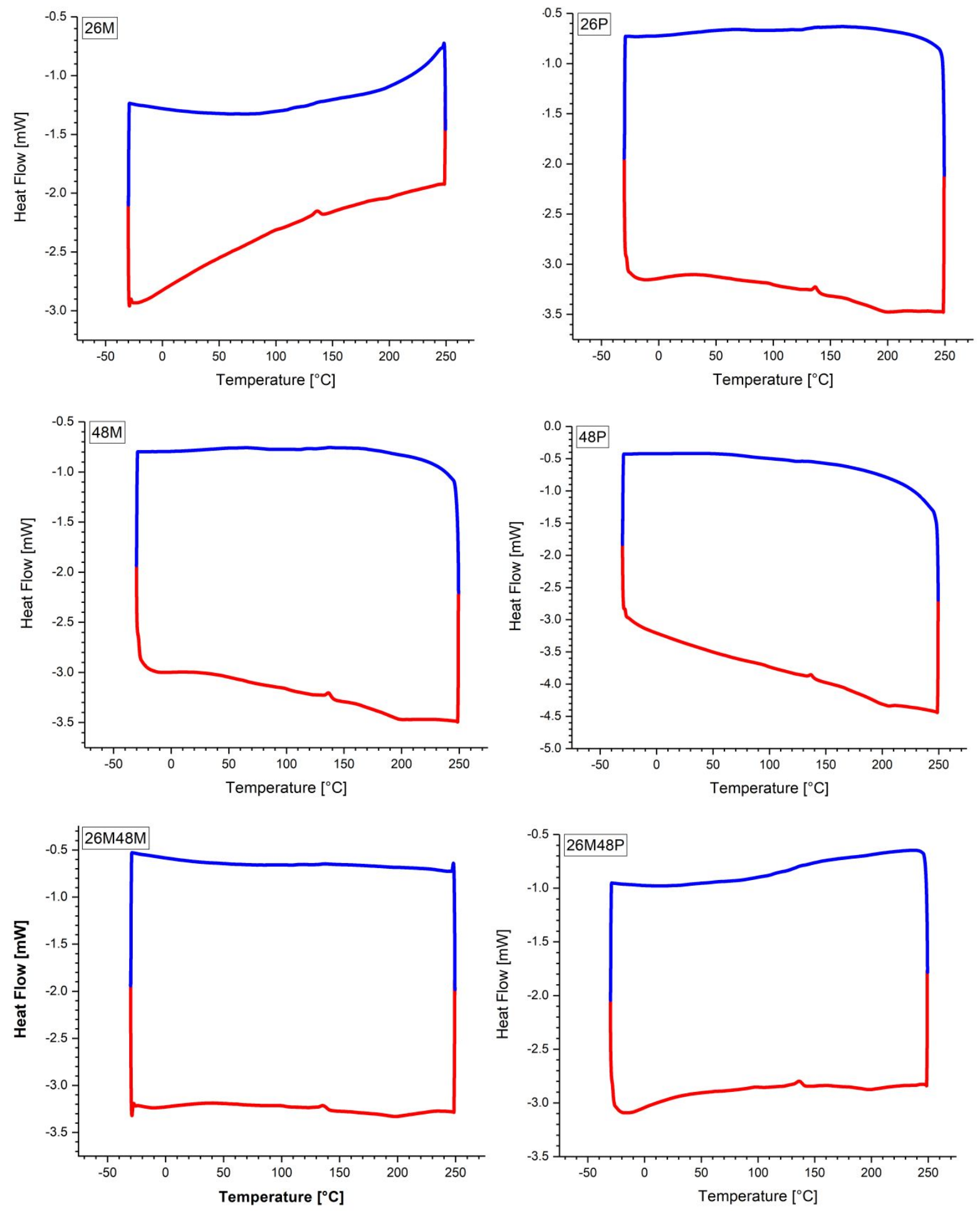

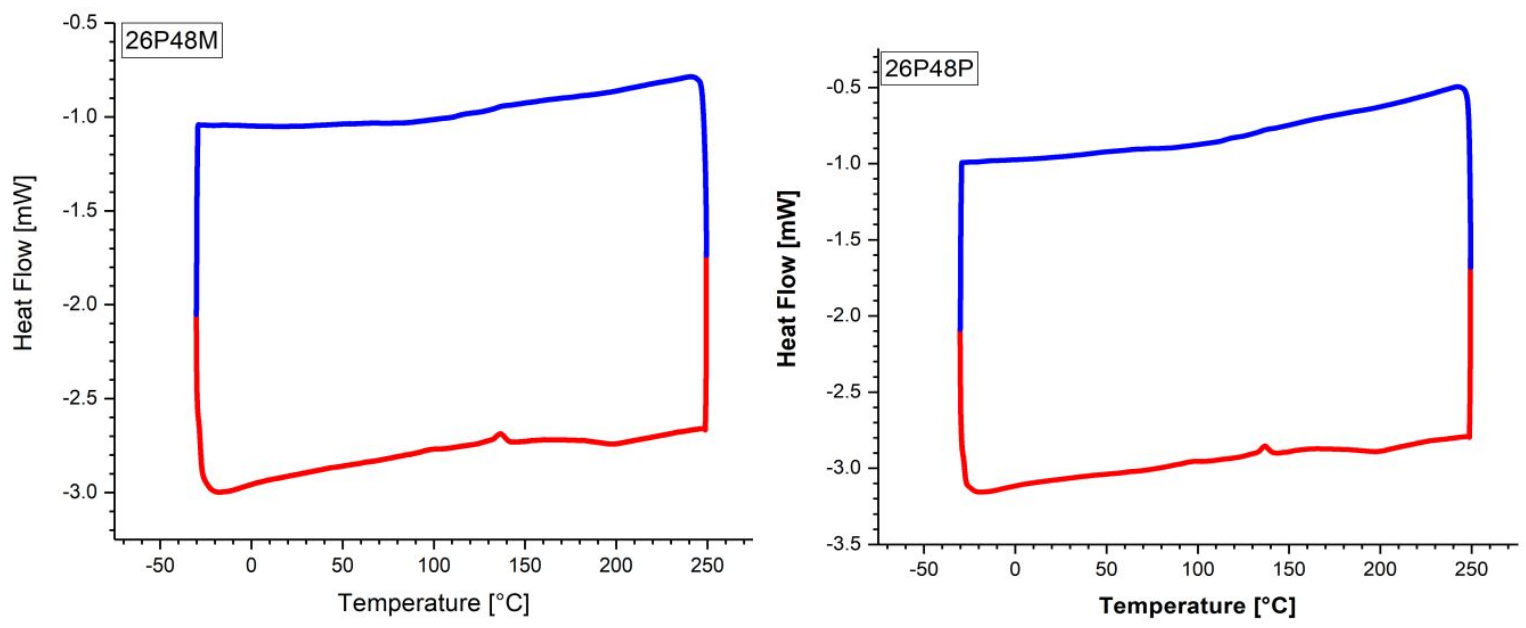

Figure S19. DSC plots (name in top left corner) for all eight compounds 


\section{UV-Vis and Photoluminescence Spectra}

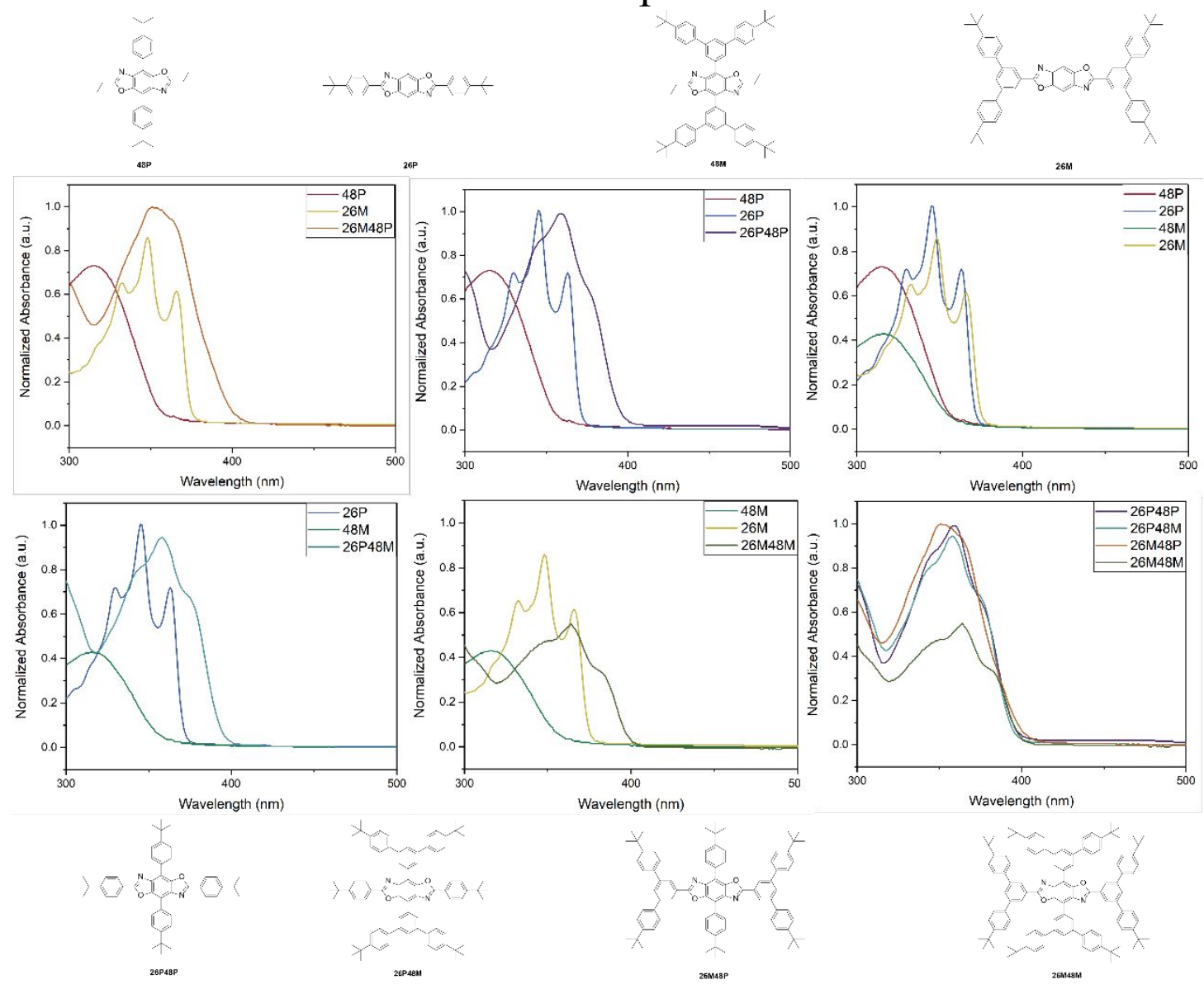

Figure S20. Solution-state UV-Vis overlays of the eight BBO small-molecules. 


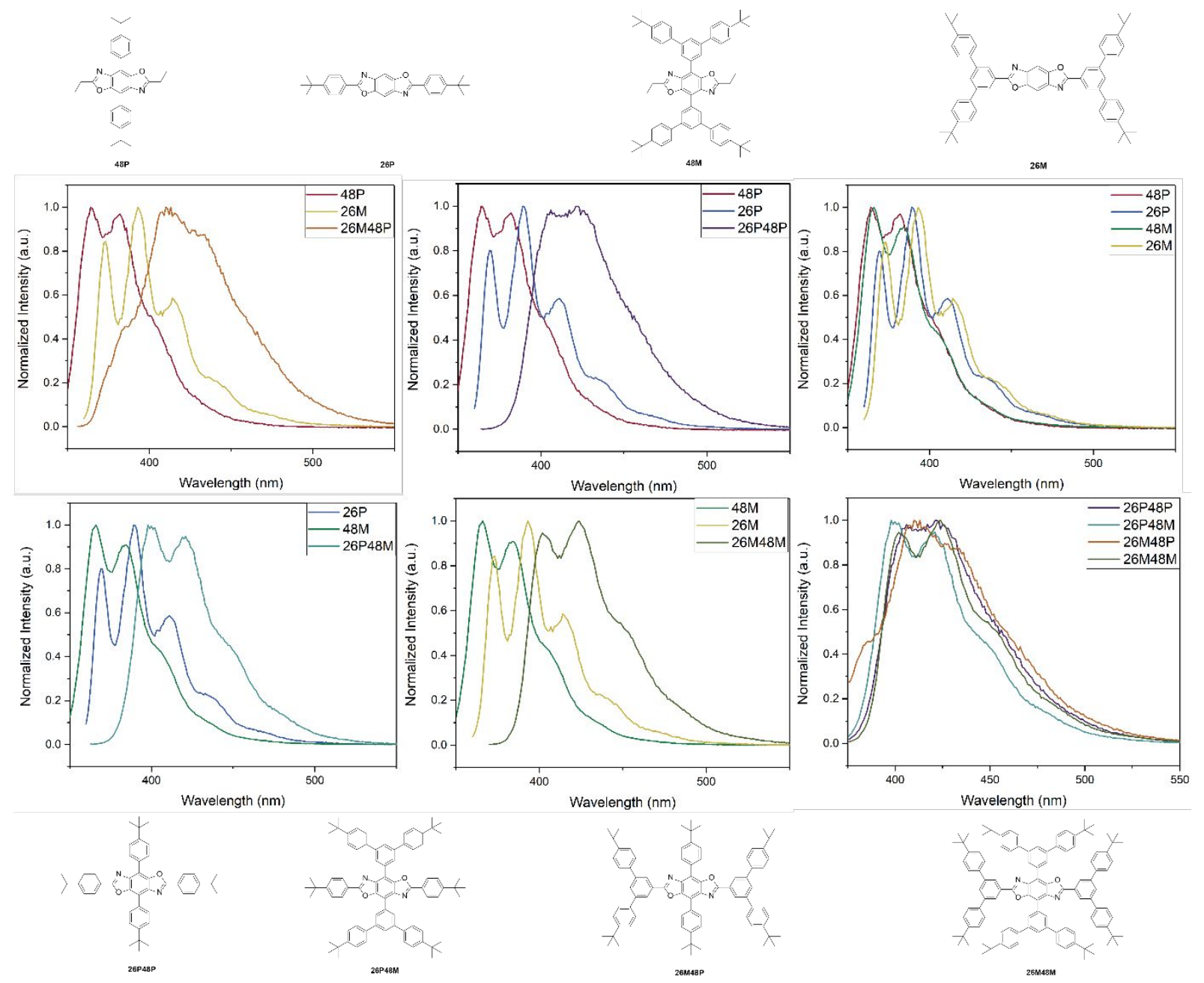

Figure S21. Solution-state PL overlays of the eight BBO small-molecules. Excitation was performed using the peak maxima from the UV-Vis spectra.

\section{DFT/TDDFT Gaussian Setups}

\section{DFT Gaussian Setups}

Gas Phase

opt freq mpw3pbe sv geom=connectivity b972

\section{Chloroform}

opt freq mpw3pbe sv scrf $=(\mathrm{cpcm}$,solvent $=$ chloroform $)$ geom $=$ connectivity

TDDFT Gaussian Setup

$\mathrm{td}=($ nstates $=15) / \mathrm{sv}$ scrf $=(\mathrm{cpcm}$, solvent $=$ chloroform $)$ geom $=$ connectivity mpw3pbe 


\section{Number of Imaginary Frequencies \& Internal Energies for all in Gas and Chloroform Phases}

\begin{tabular}{|lcccc|}
\hline & \multicolumn{2}{c}{ Gas Phase } & \multicolumn{2}{c|}{ Chloroform } \\
26M & Imaginary Freq & $\begin{array}{r}\text { Internal Energy (Hartrees) } \\
\text { Imaginary Freq }\end{array}$ & $\begin{array}{c}\text { Internal Energy (Hartrees) } \\
26 \mathrm{P}\end{array}$ \\
$48 \mathrm{M}$ & 0 & -2578.09 & 0 & -2578.10 \\
$48 \mathrm{P}$ & 0 & -1341.42 & 0 & -1341.43 \\
$26 \mathrm{M} 48 \mathrm{P}$ & 0 & -2735.08 & 0 & -2735.09 \\
$26 \mathrm{P} 48 \mathrm{P}$ & 0 & -1498.41 & 0 & -1498.42 \\
$26 \mathrm{P} 48 \mathrm{M}$ & 0 & -3353.43 & 0 & -3353.44 \\
$26 \mathrm{M} 48 \mathrm{M}$ & 0 & -2116.76 & 0 & -2116.77 \\
\hline
\end{tabular}

Table S2. Imaginary frequency counts and internal energy of all optimized compounds of interest.

\section{$26 \mathrm{M}$}

\section{Cartesian Coordinates}

Gas Phase

C $-0.04509-1.47531-0.02765$

C $-1.1765-0.67152-0.0196$

C $0.036121 .47325-0.01581$

C $1.167590 .66947-0.02322$

C $-1.167050 .75185-0.01377$

C $-3.247880 .13569-0.00688$

O - $2.51273-1.06681-0.01477$

$\mathrm{N}-2.490861 .20715-0.00645$

C $1.15804-0.75391-0.02908$

C $3.23891-0.13805-0.03231$

N $2.48172-1.20937-0.03528$

O $2.503941 .06459-0.02521$

C $4.68826-0.05375-0.0316$

C $5.338441 .1932-0.04531$

C $5.43794-1.24475-0.01457$

C $6.743961 .26289-0.04154$

H $4.7392 .10573-0.08369$

C $6.84261-1.20186-0.00997$

H $4.90232-2.196860 .02109$

C $7.439462 .57583-0.06514$

C $7.475680 .05859-0.02308$

C $7.64038-2.454960 .02018$

C 6.915233 .693250 .61868

C $8.644762 .75529-0.76975$

H $8.568610 .10294-0.01827$

C $8.84667-2.539630 .7469$

C $7.21861-3.60574-0.672$

C 7.571784 .926850 .59567

H 5.992483 .589871 .19818

C $9.299693 .99463-0.79006$

H $9.069721 .92164-1.33775$

C $9.58893-3.723690 .77791$

H $9.19575-1.675711 .32136$ 
C $7.96756-4.79021-0.63973$ H $6.29988-3.57352-1.26606$ C $8.781665 .11146-0.10779$ H 7.13047 5.761771 .14928 H $10.227114 .07852-1.36077$ C $9.1701-4.880680 .08617$ H $10.51181-3.744121 .36636$ H $7.59689-5.6502-1.2016$ C -4.697170 .050460 .00004$ C -5.447891 .240620 .02698$ C $-5.34607-1.197-0.02144$ C -6.852621 .196720 .03149$ $\mathrm{H}-4.912252 .192470 .06825$ C $-6.7515-1.26793-0.01697$ H $-4.7458-2.1086-0.0678$ C -7.652482 .448470 .06579$ C $-7.48417-0.064470 .00929$ C $-7.44578-2.58132-0.04981$ C $-7.219383 .61015-0.60794$ C -8.868962 .524030 .76989$

H -8.57698 -0.110170 .01219$ C $-8.65137-2.75674-0.75492$ C $-6.91978-3.703390 .62499$ C $-7.973854 .78608-0.5765$ H -6.28968 3.5868 -1.18497 C -9.622223 .706120 .79873$ H -9.226191 .65461 .3308$ C $-9.30486-3.99665-0.78443$ H $-9.07774-1.91926-1.31618$ C $-7.57483-4.937590 .59274$ H -5.99675 -3.6034 1.20464 C -9.19568 4.86639 0.12581 H - $7.600265 .65845-1.12185$ $\mathrm{H}-10.554163 .710261 .36825$ C $-8.78492-5.11819-0.11139$ H - $10.23259-4.07718-1.3551$ H -7.13209 -5.77633 1.1394 C -9.99446 .181690 .13022$ C -9.129837 .305530 .75486$ C $-10.362186 .56335-1.32594$ C - 11.299236 .066060 .94332 H -8.85687 7.0575 1.79776 H -8.19386 7.46488 0.18851 H -9.689178 .261130 .76118$ H - $10.983215 .77619-1.79332$ H - 10.93474 7.51094 -1.34117 H $-9.462656 .7027-1.95321$ H - 11.842277 .029310 .91434 H - 11.971465 .290990 .53028 H -11.10059 5.826942 .0047 C $-9.47282-6.49476-0.11813$ C $-10.77924-6.48721-0.93661$ C $-8.51603-7.54385-0.73835$ C $-9.81373-6.905061 .33667$ H - $11.51625-5.77277-0.52478$ H - $10.59703-6.22917-1.99652$ 


\section{Chloroform}

C $1.17232-0.66778-0.03692$

C $-0.043481 .47527-0.03698$

C - $1.172330 .66777-0.0369$

C $-1.16061-0.75503-0.03624$

C $0.04348-1.47528-0.037$

$\mathrm{N}-2.48507-1.21148-0.03325$

C -3.24424 -0.14031 -0.03217

O - $2.50991 .06048-0.03504$

C $3.244240 .1403-0.03218$

C $1.160610 .75502-0.03624$

O 2.5099-1.06049-0.03506

N $2.485071 .21146-0.03325$

H $0.07855-2.56458-0.03669$

H - $0.078552 .56457-0.03667$

C $4.694020 .05485-0.02439$

C $5.3408-1.19428-0.04123$

C $5.447491 .24397-0.01332$

C $6.74654-1.26817-0.04353$ 
H $4.73947-2.10567-0.07328$

C $6.85261 .19779-0.01367$

H $4.916842 .19924-0.02354$

C $-4.69402-0.05486-0.02435$

C -5.34079 1.19428 -0.04119

C -5.44749-1.24398 - 0.01328

C -6.74654 1.26817 -0.04349

H $-4.739472 .10565-0.07324$

C $-6.8526-1.1978-0.01363$

H $-4.91685-2.19925-0.02351$

C -7.48203 $0.06536-0.02685$

$\mathrm{H}-8.573460 .113470 .02261$

C $7.48203-0.06536-0.02689$

H $8.57346-0.113470 .02256$

C $-7.438582 .58348-0.05367$

C $-8.661072 .76286-0.72978$

C -6.895033 .702730 .61354$

C $-9.313464 .00433-0.73937$

H $-9.105081 .92868-1.28208$

C -7.549474 .938390 .6016$

H -5.95874 3.602561 .17135

C $-8.776145 .1231-0.0738$

H $-10.254514 .08722-1.28734$

H -7.09238 5.77374 1.14139

C $-7.65573-2.44810 .01141$

C $-8.89218-2.53844-0.65692$

C $-7.20867-3.591930 .70788$

C $-9.65103-3.7177-0.63009$

H -9.26462 -1.68424 -1.23104

C -7.9691 -4.76509 0.73219

H -6.26406 -3.55894 1.25966

C $-9.21079-4.860030 .06593$

H - $10.5987-3.73283-1.17268$

H -7.58439 -5.622051 .29382$

C $7.43858-2.58348-0.05371$

C $8.66108-2.76286-0.72981$

C $6.89504-3.702730 .61351$

C $9.31347-4.00433-0.73939$

H $9.10508-1.92868-1.28211$

C $7.54948-4.938380 .60158$

H $5.95875-3.602551 .17132$

C $8.77616-5.1231-0.07382$

H $10.25453-4.08722-1.28735$

H 7.0924 -5.77373 1.14138

C 7.655732 .44810 .01137

C $8.892172 .53845-0.65696$

C 7.208663 .591920 .70785

C $9.651013 .7177-0.63013$

H 9.26461 $1.68425-1.23109$

C 7.969094 .765080 .73217

H 6.264063 .558921 .25963

C 9.210774 .860030 .06589

H $10.598693 .73284-1.17271$

H 7.584385 .622041 .29381

C -9.46416 6.49932 -0.05679

C - $10.01628-6.169820 .12441$ 
C $-8.516757 .55557-0.67954$

H -8.99856 8.55223 -0.67034

H -7.56665 7.63417 -0.11981

H -8.27608 7.29825 -1.72838

C -9.782336 .894021 .40769$

H - 10.276717 .884181 .43653

H - 10.461226 .155861 .87509

H -8.86656 6.95514 2.02411

C - $10.782596 .49922-0.85579$

H -10.61667 6.24862 -1.92019

H - $11.514735 .78292-0.43856$

H - $11.239657 .50569-0.8163$

C 10.016266 .169830 .1244

C $9.46418-6.4993-0.05679$

C $-9.17328-7.31886-0.48409$

H -8.22566 -7.46763 0.06573

H $-9.74004-8.26909-0.44564$

H -8.92577 -7.10744 - 1.54156

C - $10.34807-6.501541 .60141$

H - $10.95481-5.696752 .05792$

H - 10.92524 -7.44446 1.65961

H -9.4334 -6.62737 2.20959

C $-11.34077-6.07244-0.65868$

H - $11.99883-5.28118-0.2536$

H -11.16864 -5.868 -1.7319

H - $11.88608-7.03184-0.58427$

C $8.51682-7.55556-0.6796$

H $8.99864-8.55222-0.67037$

H 7.56669 -7.63418 -0.11991

H $8.2762-7.29823-1.72844$

C $9.78228-6.894021 .40771$

H $10.27665-7.884171 .43657$

H $10.46114-6.155861 .87515$

H $8.86647-6.955142 .02408$

C $10.78266-6.49919-0.85571$

H $10.61679-6.24858-1.92011$

H 11.51477 -5.7829 -0.43843

H $11.23972-7.50567-0.81622$

C $11.340756 .07246-0.65868$

H $11.998795 .28117-0.25365$

H $11.168635 .86809-1.73191$

H $11.886087 .03185-0.58421$

C 10.348046 .501521 .6014

H 10.925197 .444451 .65962

H 9.433366 .627322 .20959

H 10.954795 .696732 .0579

C $9.173267 .31887-0.48408$

H $9.740028 .26911-0.44561$

H $8.925767 .10747-1.54157$

H 8.225647 .467630 .06572

\section{Energy Levels}

Gas Phase

Alpha occ. eigenvalues -- -19.20920 -19.20919 -14.33307 -14.33304 -10.28602

Alpha occ. eigenvalues -- -10.28601 -10.25493 -10.25486 -10.22898 -10.22891 
Alpha occ. eigenvalues -- -10.20520 -10.20520 -10.20329 -10.20320 -10.19785 Alpha occ. eigenvalues -- -10.19782 -10.19778 -10.19775 -10.19727 -10.19720 Alpha occ. eigenvalues -- -10.19718 -10.19717 -10.19017-10.19015 -10.18925 Alpha occ. eigenvalues -- -10.18916 -10.18744 -10.18740 -10.18714 -10.18711 Alpha occ. eigenvalues -- -10.18630 -10.18630 -10.18603 -10.18597-10.18562 Alpha occ. eigenvalues -- -10.18557 -10.17912 -10.17909-10.17886 -10.17884 Alpha occ. eigenvalues -- -10.17866 -10.17813 -10.17791 -10.17737 -10.17686 Alpha occ. eigenvalues -- -10.17684 -10.17622 -10.17617-10.17615 -10.17579 Alpha occ. eigenvalues -- -10.17538 -10.17494 -10.16092 -10.16089-10.16076 Alpha occ. eigenvalues -- -10.16073 -10.16069 -10.16066 -10.16043-10.16029 Alpha occ. eigenvalues -- $-10.16019-10.16014-10.16000-10.15992-1.14008$ Alpha occ. eigenvalues -- $-1.13905-0.94926-0.94856-0.89175-0.88774$ Alpha occ. eigenvalues -- $-0.87741-0.87047-0.87045-0.86314-0.86035$ Alpha occ. eigenvalues -- $-0.82715-0.82713-0.82460-0.82123-0.80340$ Alpha occ. eigenvalues -- $-0.78838-0.78335-0.78275-0.77712-0.75228$ Alpha occ. eigenvalues -- $-0.75014-0.74956-0.74920-0.74823-0.73438$ Alpha occ. eigenvalues -- $-0.72660-0.72128-0.72060-0.68992-0.68686$ Alpha occ. eigenvalues -- $-0.68657-0.68615-0.68476-0.68451-0.68448$ Alpha occ. eigenvalues -- -0.68397 -0.68385 -0.65763 -0.64901 -0.64558 Alpha occ. eigenvalues -- -0.63637 -0.63389 -0.62698 -0.62556 -0.60957 Alpha occ. eigenvalues -- $-0.60938-0.60852-0.60422-0.59576-0.58520$ Alpha occ. eigenvalues -- $-0.58405-0.58005-0.56037-0.55661-0.55607$ Alpha occ. eigenvalues -- $-0.55516-0.55503-0.55457-0.54286-0.51469$ Alpha occ. eigenvalues -- $-0.50369-0.50335-0.50064-0.49898-0.49845$ Alpha occ. eigenvalues -- $-0.49766-0.49092-0.48669-0.48472-0.48138$ Alpha occ. eigenvalues -- $-0.47630-0.47263-0.47245-0.47103-0.46743$ Alpha occ. eigenvalues -- $-0.45803-0.45682-0.45609-0.45522-0.45229$ Alpha occ. eigenvalues -- $-0.45194-0.44912-0.44845-0.44811-0.44700$ Alpha occ. eigenvalues -- $-0.44686-0.44634-0.44622-0.44487-0.43287$ Alpha occ. eigenvalues -- $-0.43233-0.43123-0.43061-0.42623-0.42600$ Alpha occ. eigenvalues -- $-0.42473-0.42414-0.41038-0.40458-0.40290$ Alpha occ. eigenvalues -- -0.40160 -0.40090 -0.40076 -0.39998 -0.39954 Alpha occ. eigenvalues -- -0.39804 -0.39675 -0.39488 -0.38308 -0.38294 Alpha occ. eigenvalues -- $-0.38207-0.38189-0.37796-0.37663-0.37595$ Alpha occ. eigenvalues -- $-0.37391-0.37209-0.37180-0.37023-0.36964$ Alpha occ. eigenvalues -- $-0.36907-0.36892-0.36780-0.36438-0.36377$ Alpha occ. eigenvalues -- $-0.36016-0.35878-0.35464-0.35449-0.35396$ Alpha occ. eigenvalues -- -0.35369-0.34175 -0.33942 -0.33932 -0.33767 Alpha occ. eigenvalues -- $-0.33400-0.33220-0.33188-0.33083-0.33048$ Alpha occ. eigenvalues -- $-0.32412-0.32401-0.32338-0.32318-0.30245$ Alpha occ. eigenvalues -- $-0.29631-0.29315-0.29200-0.29176-0.27578$ Alpha occ. eigenvalues -- $-0.26304-0.26302-0.26208-0.26205-0.25862$ Alpha occ. eigenvalues -- $-0.24726-0.24023-0.23183-0.23172-0.22598$ Alpha virt. eigenvalues -- $-0.08357-0.05597-0.04654-0.04629-0.02683$ Alpha virt. eigenvalues -- $-0.01679-0.01409-0.01376-0.01318-0.01246$ Alpha virt. eigenvalues -- -0.010810 .011570 .024800 .025320 .04951$ Alpha virt. eigenvalues -- 0.053930 .054000 .054450 .054830 .05913 Alpha virt. eigenvalues -- 0.080130 .080790 .084010 .084790 .08525 Alpha virt. eigenvalues -- 0.086540 .089780 .091140 .093230 .09777 Alpha virt. eigenvalues -- 0.099700 .101160 .101620 .101730 .10544 Alpha virt. eigenvalues -- 0.106280 .108790 .110640 .114800 .11487 Alpha virt. eigenvalues -- 0.115550 .115660 .117480 .118350 .12152 Alpha virt. eigenvalues -- 0.121870 .123430 .124100 .124390 .12448 Alpha virt. eigenvalues -- 0.125050 .126090 .129320 .129660 .13331 Alpha virt. eigenvalues -- 0.136390 .139590 .144340 .152300 .15373 Alpha virt. eigenvalues -- 0.155850 .158060 .159360 .162730 .16458 
Alpha virt. eigenvalues -- 0.171150 .171540 .171820 .171990 .17279 Alpha virt. eigenvalues -- 0.173750 .175120 .176330 .177120 .17812 Alpha virt. eigenvalues -- 0.180220 .183660 .184940 .188620 .18885 Alpha virt. eigenvalues -- 0.189420 .189930 .191120 .192030 .19241 Alpha virt. eigenvalues -- 0.193010 .194600 .199280 .202690 .20329 Alpha virt. eigenvalues -- 0.205100 .206150 .217660 .218850 .22288 Alpha virt. eigenvalues -- 0.224570 .227360 .238280 .240100 .24039 Alpha virt. eigenvalues -- 0.240830 .241090 .246670 .246920 .24725 Alpha virt. eigenvalues -- 0.247580 .256940 .258020 .267340 .26881 Alpha virt. eigenvalues -- 0.270110 .270850 .271560 .277200 .27862 Alpha virt. eigenvalues -- 0.280840 .284860 .288110 .293880 .29761 Alpha virt. eigenvalues -- 0.299870 .302820 .303660 .306910 .30783 Alpha virt. eigenvalues -- 0.309710 .314030 .318260 .321960 .32816 Alpha virt. eigenvalues -- 0.329460 .330190 .332620 .336460 .33808 Alpha virt. eigenvalues -- 0.340440 .343420 .348630 .349430 .35297 Alpha virt. eigenvalues -- 0.358180 .360110 .366000 .368740 .37107 Alpha virt. eigenvalues -- 0.372810 .374800 .382240 .384910 .39534 Alpha virt. eigenvalues -- 0.396230 .397310 .399180 .404890 .41039 Alpha virt. eigenvalues -- 0.418900 .418950 .419430 .419530 .42334 Alpha virt. eigenvalues -- 0.426580 .433010 .434240 .436590 .43776 Alpha virt. eigenvalues -- 0.438460 .439150 .440530 .442150 .44286 Alpha virt. eigenvalues -- 0.445680 .449780 .451760 .453800 .45484 Alpha virt. eigenvalues -- 0.457040 .461950 .463350 .463970 .46636 Alpha virt. eigenvalues -- 0.466860 .468880 .473240 .473450 .47734 Alpha virt. eigenvalues -- 0.478410 .479430 .481120 .484390 .48630 Alpha virt. eigenvalues -- 0.490560 .491120 .491990 .492380 .49574 Alpha virt. eigenvalues -- 0.498080 .505250 .506070 .508400 .50916 Alpha virt. eigenvalues -- 0.516880 .519680 .523780 .526580 .53222 Alpha virt. eigenvalues -- 0.533260 .535300 .541190 .549750 .55495 Alpha virt. eigenvalues -- 0.555440 .558340 .560070 .561880 .56341 Alpha virt. eigenvalues -- 0.565570 .565860 .572460 .573460 .57613 Alpha virt. eigenvalues -- 0.576890 .579630 .581270 .583250 .58883 Alpha virt. eigenvalues -- 0.590170 .596340 .596790 .603940 .60630 Alpha virt. eigenvalues -- 0.607800 .612680 .613860 .615030 .61695 Alpha virt. eigenvalues -- 0.617920 .618740 .620640 .622440 .62352 Alpha virt. eigenvalues -- 0.624460 .625420 .631890 .635610 .63785 Alpha virt. eigenvalues -- 0.638870 .641270 .644340 .644980 .64763 Alpha virt. eigenvalues -- 0.650120 .659610 .660940 .664500 .66552 Alpha virt. eigenvalues -- 0.666470 .667590 .670380 .671000 .67149 Alpha virt. eigenvalues -- 0.671870 .673660 .674220 .675540 .67646 Alpha virt. eigenvalues -- 0.676960 .678270 .678660 .680310 .68356 Alpha virt. eigenvalues -- 0.685890 .686250 .686630 .687010 .68756 Alpha virt. eigenvalues -- 0.689350 .689700 .691750 .692670 .69293 Alpha virt. eigenvalues -- 0.694200 .696060 .697480 .698340 .69914 Alpha virt. eigenvalues -- 0.699410 .700640 .704400 .705050 .70862 Alpha virt. eigenvalues -- 0.709340 .710920 .711860 .714580 .71868 Alpha virt. eigenvalues -- 0.720040 .720450 .721120 .722960 .72338 Alpha virt. eigenvalues -- 0.724250 .725440 .726000 .726800 .72925 Alpha virt. eigenvalues -- 0.730320 .731170 .732130 .732330 .73367 Alpha virt. eigenvalues -- 0.734790 .736930 .741560 .741810 .74375 Alpha virt. eigenvalues -- 0.744830 .745700 .746530 .747410 .74811 Alpha virt. eigenvalues -- 0.749060 .753690 .754230 .755350 .75711 Alpha virt. eigenvalues -- 0.757380 .759380 .760640 .762410 .76520 Alpha virt. eigenvalues -- 0.766390 .767470 .771050 .773830 .77439 Alpha virt. eigenvalues -- 0.776550 .778840 .781120 .783560 .78709 Alpha virt. eigenvalues -- 0.791850 .794990 .797110 .799540 .80027 
Alpha virt. eigenvalues -- 0.801920 .802990 .810930 .811430 .81448 Alpha virt. eigenvalues -- 0.818740 .821780 .825590 .825960 .82906 Alpha virt. eigenvalues -- 0.837220 .838460 .840990 .842460 .84353 Alpha virt. eigenvalues -- 0.844070 .844580 .845450 .846320 .84929 Alpha virt. eigenvalues -- 0.850870 .852210 .855540 .866850 .86870 Alpha virt. eigenvalues -- 0.872660 .876480 .877660 .881380 .88369 Alpha virt. eigenvalues -- 0.892040 .899040 .909620 .910530 .91219 Alpha virt. eigenvalues -- 0.913320 .914490 .920180 .927760 .93966 Alpha virt. eigenvalues -- 0.941320 .947230 .956760 .984150 .99519 Alpha virt. eigenvalues -- 1.000441 .006611 .010361 .013031 .01577 Alpha virt. eigenvalues -- 1.024911 .027311 .033351 .034621 .04063 Alpha virt. eigenvalues -- 1.046031 .047461 .049561 .059931 .06931 Alpha virt. eigenvalues -- 1.075851 .082121 .090331 .093141 .09396 Alpha virt. eigenvalues -- 1.096851 .099081 .101491 .103141 .10751 Alpha virt. eigenvalues -- 1.110881 .122921 .127371 .130731 .13547 Alpha virt. eigenvalues -- 1.136021 .137501 .139591 .140671 .14202 Alpha virt. eigenvalues -- 1.144011 .147831 .169771 .171151 .18631 Alpha virt. eigenvalues -- 1.187691 .191901 .206461 .228261 .23250 Alpha virt. eigenvalues -- 1.248251 .255081 .259331 .262481 .27460 Alpha virt. eigenvalues -- 1.296351 .299301 .304431 .309291 .31777 Alpha virt. eigenvalues -- 1.322441 .329191 .333501 .342451 .35141 Alpha virt. eigenvalues -- 1.356121 .358121 .362721 .402671 .41335 Alpha virt. eigenvalues -- 1.422311 .432091 .434311 .453081 .51843 Alpha virt. eigenvalues -- 1.545681 .553511 .562851 .584611 .58463 Alpha virt. eigenvalues -- 1.588331 .588681 .708351 .769631 .80397 Alpha virt. eigenvalues -- 1.82759

\section{Chloroform}

Alpha occ. eigenvalues -- -19.21752 -19.21752 -14.34244 -14.34242 -10.29521 Alpha occ. eigenvalues -- -10.29520 -10.26226 -10.26219-10.23673 -10.23668 Alpha occ. eigenvalues -- -10.21291 -10.21291 -10.20963 -10.20953 -10.20422 Alpha occ. eigenvalues -- -10.20422 -10.20402 -10.20402 -10.19707 -10.19707 Alpha occ. eigenvalues -- -10.19689 -10.19689 -10.19475 -10.19475 -10.19442 Alpha occ. eigenvalues -- -10.19441 -10.19403 -10.19403 -10.19299-10.19299 Alpha occ. eigenvalues -- -10.19162 -10.19162 -10.19018 -10.19018 -10.18990 Alpha occ. eigenvalues -- -10.18989 -10.18266 -10.18266 -10.18240 -10.18240 Alpha occ. eigenvalues -- -10.18223 -10.18223 -10.18214 -10.18214 -10.17943 Alpha occ. eigenvalues -- -10.17943 -10.17902 -10.17902 -10.17877 -10.17877 Alpha occ. eigenvalues -- -10.17851 -10.17851 -10.15921 -10.15921 -10.15910 Alpha occ. eigenvalues -- -10.15910 -10.15901 -10.15901-10.15892 -10.15892 Alpha occ. eigenvalues -- -10.15804 -10.15804 -10.15788 -10.15788 -1.14845 Alpha occ. eigenvalues -- $-1.14744-0.95719-0.95648-0.89853-0.89383$ Alpha occ. eigenvalues -- $-0.88366-0.87393-0.87393-0.86697-0.86443$ Alpha occ. eigenvalues -- $-0.82855-0.82855-0.82762-0.82292-0.80814$ Alpha occ. eigenvalues -- $-0.79382-0.78780-0.78663-0.78346-0.75757$ Alpha occ. eigenvalues -- $-0.75316-0.75276-0.75268-0.75206-0.73789$ Alpha occ. eigenvalues -- $-0.73107-0.72567-0.72493-0.69591-0.68511$ Alpha occ. eigenvalues -- $-0.68503-0.68489-0.68426-0.68299-0.68299$ Alpha occ. eigenvalues -- $-0.68281-0.68281-0.66431-0.65496-0.65039$ Alpha occ. eigenvalues -- $-0.64120-0.63946-0.63261-0.62917-0.61270$ Alpha occ. eigenvalues -- -0.61236 -0.61129 -0.60811 -0.60035 -0.59009 Alpha occ. eigenvalues -- $-0.58767-0.58564-0.56767-0.56115-0.55743$ Alpha occ. eigenvalues -- $-0.55629-0.55625-0.55604-0.54698-0.51978$ Alpha occ. eigenvalues -- $-0.50691-0.50542-0.50514-0.50513-0.50274$ Alpha occ. eigenvalues -- $-0.50246-0.49523-0.49308-0.49308-0.48521$ 
Alpha occ. eigenvalues -- -0.48139 -0.47546 -0.47544 -0.47395 -0.47110 Alpha occ. eigenvalues -- $-0.46024-0.45958-0.45826-0.45798-0.45495$ Alpha occ. eigenvalues -- $-0.45342-0.45228-0.45122-0.45080-0.45073$ Alpha occ. eigenvalues -- $-0.44540-0.44540-0.44520-0.44518-0.43442$ Alpha occ. eigenvalues -- $-0.43438-0.43234-0.43225-0.42687-0.42659$ Alpha occ. eigenvalues -- $-0.42527-0.42521-0.41754-0.41070-0.40747$ Alpha occ. eigenvalues -- $-0.40629-0.40376-0.40188-0.40059-0.40030$ Alpha occ. eigenvalues -- $-0.40000-0.39898-0.39745-0.38498-0.38259$ Alpha occ. eigenvalues -- $-0.38246-0.38130-0.38123-0.38076-0.37772$ Alpha occ. eigenvalues -- $-0.37681-0.37548-0.37536-0.37443-0.36854$ Alpha occ. eigenvalues -- $-0.36828-0.36771-0.36768-0.36739-0.36686$ Alpha occ. eigenvalues -- $-0.36575-0.36418-0.35730-0.35714-0.35583$ Alpha occ. eigenvalues -- -0.35535 -0.34707 -0.33860 -0.33859-0.33767 Alpha occ. eigenvalues -- -0.33561 -0.33229 -0.33221 -0.33139-0.33138 Alpha occ. eigenvalues -- $-0.32373-0.32366-0.32339-0.32338-0.30945$ Alpha occ. eigenvalues -- $-0.30741-0.30401-0.29717-0.29710-0.28319$ Alpha occ. eigenvalues -- $-0.26717-0.26717-0.26683-0.26681-0.26588$ Alpha occ. eigenvalues -- $-0.25334-0.24581-0.23647-0.23637-0.23315$ Alpha virt. eigenvalues -- $-0.09122-0.06394-0.05280-0.05258-0.03386$ Alpha virt. eigenvalues -- $-0.02334-0.01808-0.01790-0.01789-0.01715$ Alpha virt. eigenvalues -- -0.017090 .004400 .022070 .022300 .04245$ Alpha virt. eigenvalues -- 0.050830 .057010 .057120 .057700 .05771 Alpha virt. eigenvalues -- 0.074230 .074470 .079210 .084800 .08653 Alpha virt. eigenvalues -- 0.087940 .088010 .093790 .099880 .10076 Alpha virt. eigenvalues -- 0.100850 .102700 .104150 .104210 .10511 Alpha virt. eigenvalues -- 0.105850 .112090 .112320 .114460 .11485 Alpha virt. eigenvalues -- 0.116130 .116570 .117070 .117510 .11894 Alpha virt. eigenvalues -- 0.119030 .122790 .125460 .126960 .12697 Alpha virt. eigenvalues -- 0.128540 .128660 .134100 .134260 .13459 Alpha virt. eigenvalues -- 0.137590 .139520 .139580 .145420 .15594 Alpha virt. eigenvalues -- 0.157770 .158990 .159010 .162180 .16273 Alpha virt. eigenvalues -- 0.168070 .171800 .173290 .174350 .17438 Alpha virt. eigenvalues -- 0.175670 .175910 .175970 .176490 .17672 Alpha virt. eigenvalues -- 0.177870 .185450 .189860 .189880 .19047 Alpha virt. eigenvalues -- 0.191140 .191190 .191440 .191830 .19347 Alpha virt. eigenvalues -- 0.194400 .194460 .195430 .203540 .20379 Alpha virt. eigenvalues -- 0.203830 .204830 .217050 .218210 .21915 Alpha virt. eigenvalues -- 0.223530 .224410 .231920 .238270 .23875 Alpha virt. eigenvalues -- 0.239370 .239730 .246580 .246610 .24719 Alpha virt. eigenvalues -- 0.247530 .251490 .252830 .262050 .26420 Alpha virt. eigenvalues -- 0.264310 .264570 .265120 .272590 .27295 Alpha virt. eigenvalues -- 0.274620 .278660 .284880 .291960 .29325 Alpha virt. eigenvalues -- 0.296010 .296300 .297560 .301040 .30137 Alpha virt. eigenvalues -- 0.302980 .308730 .316810 .318890 .32412 Alpha virt. eigenvalues -- 0.324980 .325010 .326260 .329770 .33213 Alpha virt. eigenvalues -- 0.333790 .339060 .342080 .344640 .34476 Alpha virt. eigenvalues -- 0.353420 .355580 .357600 .358740 .36338 Alpha virt. eigenvalues -- 0.372140 .373750 .374180 .379140 .38991 Alpha virt. eigenvalues -- 0.390710 .393080 .394240 .399750 .40583 Alpha virt. eigenvalues -- 0.417140 .419460 .420590 .420650 .42101 Alpha virt. eigenvalues -- 0.421480 .428930 .429870 .430290 .43111 Alpha virt. eigenvalues -- 0.432720 .436640 .436720 .438110 .44330 Alpha virt. eigenvalues -- 0.444860 .447830 .448750 .450430 .45391 Alpha virt. eigenvalues -- 0.454290 .456530 .456720 .461540 .46174 Alpha virt. eigenvalues -- 0.465430 .465710 .465770 .468700 .47132 Alpha virt. eigenvalues -- 0.472370 .475710 .478750 .481820 .48219 
Alpha virt. eigenvalues -- 0.483710 .484590 .488930 .491170 .49308 Alpha virt. eigenvalues -- 0.494760 .501650 .502930 .505620 .50639 Alpha virt. eigenvalues -- 0.511040 .511850 .519180 .522390 .52644 Alpha virt. eigenvalues -- 0.526760 .529380 .537520 .545960 .54644 Alpha virt. eigenvalues -- 0.548780 .553910 .557400 .557430 .55913 Alpha virt. eigenvalues -- 0.563490 .565400 .565880 .565950 .56770 Alpha virt. eigenvalues -- 0.576570 .577730 .578650 .581360 .58791 Alpha virt. eigenvalues -- 0.588310 .589570 .593110 .599860 .60114 Alpha virt. eigenvalues -- 0.605880 .606320 .608120 .608290 .61418 Alpha virt. eigenvalues -- 0.614450 .616910 .618660 .622020 .62238 Alpha virt. eigenvalues -- 0.623650 .628290 .629890 .630500 .63084 Alpha virt. eigenvalues -- 0.631990 .632600 .642600 .642710 .64864 Alpha virt. eigenvalues -- 0.648720 .657420 .657860 .658100 .65921 Alpha virt. eigenvalues -- 0.664120 .667190 .667310 .667350 .67238 Alpha virt. eigenvalues -- 0.672510 .672940 .673910 .674350 .67454 Alpha virt. eigenvalues -- 0.675030 .675690 .676800 .678030 .68099 Alpha virt. eigenvalues -- 0.681690 .684060 .685860 .686070 .68705 Alpha virt. eigenvalues -- 0.687470 .688840 .689600 .690800 .69101 Alpha virt. eigenvalues -- 0.693570 .695560 .696580 .698430 .69868 Alpha virt. eigenvalues -- 0.701570 .701780 .706420 .706730 .70967 Alpha virt. eigenvalues -- 0.709800 .712620 .712780 .713710 .71495 Alpha virt. eigenvalues -- 0.717530 .717740 .719070 .723260 .72367 Alpha virt. eigenvalues -- 0.725300 .725580 .726610 .727790 .72853 Alpha virt. eigenvalues -- 0.728720 .728970 .731770 .733790 .73423 Alpha virt. eigenvalues -- 0.736430 .736480 .738170 .738640 .73903 Alpha virt. eigenvalues -- 0.739970 .740120 .742810 .745020 .74652 Alpha virt. eigenvalues -- 0.749380 .749660 .752570 .752590 .75474 Alpha virt. eigenvalues -- 0.755820 .756890 .757200 .762670 .76333 Alpha virt. eigenvalues -- 0.763670 .768670 .769630 .772010 .77306 Alpha virt. eigenvalues -- 0.774680 .775250 .777740 .780660 .78088 Alpha virt. eigenvalues -- 0.784490 .791600 .793810 .798980 .79975 Alpha virt. eigenvalues -- 0.799790 .799990 .809810 .810350 .81602 Alpha virt. eigenvalues -- 0.816940 .818460 .819700 .824630 .82689 Alpha virt. eigenvalues -- 0.826900 .828840 .833600 .835810 .83727 Alpha virt. eigenvalues -- 0.841280 .845220 .846280 .846300 .84692 Alpha virt. eigenvalues -- 0.846960 .851940 .852660 .864290 .87113 Alpha virt. eigenvalues -- 0.872610 .873920 .875730 .877750 .88199 Alpha virt. eigenvalues -- 0.887240 .894340 .900270 .902780 .90605 Alpha virt. eigenvalues -- 0.907420 .907530 .911700 .917580 .93366 Alpha virt. eigenvalues -- 0.937100 .942580 .950150 .979430 .98686 Alpha virt. eigenvalues -- 0.999381 .006651 .009061 .009491 .01501 Alpha virt. eigenvalues -- 1.017101 .017701 .023081 .029131 .03457 Alpha virt. eigenvalues -- 1.042591 .043051 .048891 .060581 .07694 Alpha virt. eigenvalues -- 1.077781 .080941 .084521 .092381 .09389 Alpha virt. eigenvalues -- 1.094861 .095671 .102741 .111831 .11223 Alpha virt. eigenvalues -- 1.113721 .125331 .126821 .129291 .13153 Alpha virt. eigenvalues -- 1.132471 .134781 .136481 .136701 .14036 Alpha virt. eigenvalues -- 1.150091 .152871 .166801 .169241 .18010 Alpha virt. eigenvalues -- 1.181301 .191631 .201821 .224711 .23047 Alpha virt. eigenvalues -- 1.240301 .243461 .259561 .267481 .27500 Alpha virt. eigenvalues -- 1.290651 .291981 .299981 .300271 .31350 Alpha virt. eigenvalues -- 1.316221 .327761 .332251 .340211 .35180 Alpha virt. eigenvalues -- 1.355021 .356241 .360301 .397781 .40740 Alpha virt. eigenvalues -- 1.417951 .426921 .427581 .445021 .51051 Alpha virt. eigenvalues -- 1.536631 .542631 .553101 .585601 .58568 Alpha virt. eigenvalues -- 1.586781 .587021 .701581 .762591 .79613 
Alpha virt. eigenvalues -- 1.81971

Comparison of Experimental and DFT UV-Vis

\section{Gas Phase}

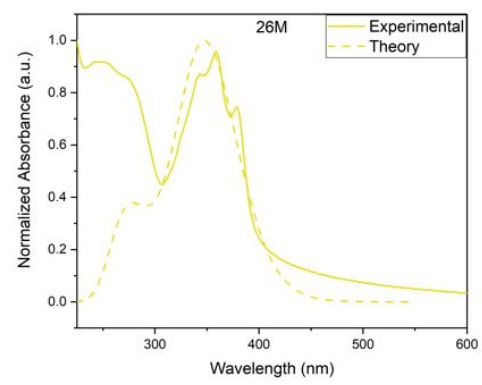

Chloroform

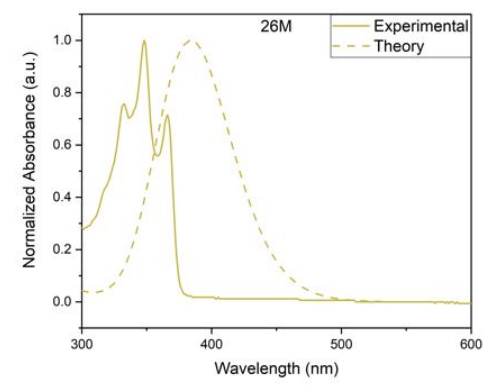

Figure S22. Gas phase and chloroform overlays of computation and experimental data for 26M.

\section{Lowest 15 Excited States}

\begin{tabular}{|c|c|c|c|c|c|c|}
\hline \multicolumn{4}{|c|}{ Gas Phase } & \multicolumn{3}{|c|}{ Chloroform } \\
\hline$\frac{\text { Excited }}{\text { state }}$ & $\frac{\text { Energy }}{(\mathrm{eV})}$ & $\frac{\text { Wavelength }}{(\mathrm{nm})}$ & $f$ & $\frac{\text { Energy }}{(\mathrm{eV})}$ & $\frac{\text { Wavelength }}{(\mathrm{nm})}$ & $f$ \\
\hline 1 & 3.54 & 259.19 & 1.68 & 3.46 & 358.78 & 1.74 \\
\hline 2 & 3.60 & 344.14 & 0.02 & 3.52 & 352.47 & 0.00 \\
\hline 3 & 3.61 & 343.60 & 0.20 & 3.52 & 352.05 & 0.24 \\
\hline 4 & 3.91 & 316.71 & 0.00 & 3.85 & 321.74 & 0.00 \\
\hline 5 & 4.06 & 305.57 & 0.30 & 4.00 & 309.69 & 0.33 \\
\hline 6 & 4.25 & 291.88 & 0.03 & 4.22 & 293.92 & 0.06 \\
\hline 7 & 4.29 & 289.16 & 0.00 & 4.23 & 293.16 & 0.00 \\
\hline 8 & 4.29 & 288.83 & 0.01 & 4.24 & 292.73 & 0.00 \\
\hline 9 & 4.32 & 286.88 & 0.00 & 4.27 & 290.20 & 0.00 \\
\hline 10 & 4.46 & 277.96 & 0.00 & 4.38 & 282.97 & 0.00 \\
\hline 11 & 4.46 & 277.90 & 0.00 & 4.38 & 282.97 & 0.00 \\
\hline 12 & 4.89 & 276.37 & 0.00 & 4.39 & 282.37 & 0.00 \\
\hline 13 & 4.49 & 276.31 & 0.00 & 4.39 & 282.36 & 0.00 \\
\hline 14 & 4.55 & 272.38 & 0.37 & 4.49 & 275.84 & 1.02 \\
\hline 15 & 4.56 & 272.19 & 0.26 & 4.50 & 275.66 & 0.00 \\
\hline
\end{tabular}

Table S3. Gas phase and chloroform excitation energies, wavelengths, and oscillator strengths for first 15 excited state of $26 \mathrm{M}$.

\section{Geometric Images}

Gas Phase 

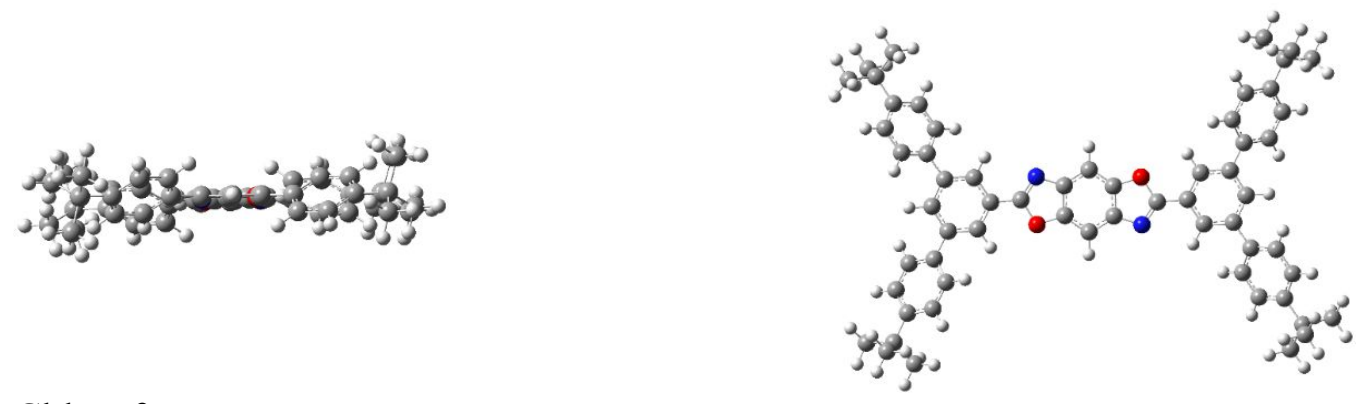

Chloroform
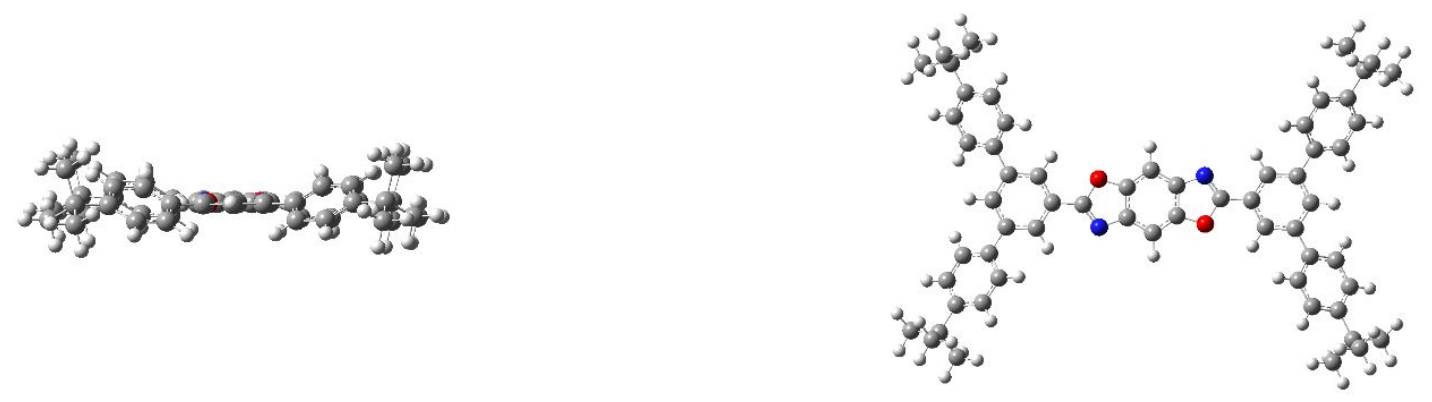

Figure S23. Geometric optimization images in gas phase and chloroform for 26M.

26P

\section{Cartesian Coordinates}

\section{Gas Phase}

C $0.04966-1.47369-0.00001$

C $1.17636-0.66318-0.00001$

C $-0.049661 .47368-0.00001$

C $-1.176360 .66318-0.00001$

C $1.15830 .76014-0.00001$

C $3.24330 .15702-0.00001$

O $2.51492-1.05054-0.00001$

N $2.479031 .22401-0.00001$

C $-1.1583-0.76014-0.00001$

C $-3.2433-0.15702-0.00001$

$\mathrm{N}-2.47903-1.22401-0.00001$

O -2.51492 $1.05054-0.00001$

$\mathrm{H}-0.086222 .56276-0.00001$

H $0.08622-2.56276-0.00001$

C $4.690370 .07981-0.00001$

C $5.36158-1.156010 .00002$

C $5.450761 .26799-0.00003$

C $6.761-1.201080 .00003$

H $4.78184-2.082650 .00004$

C $6.843751 .20912-0.00003$

H $4.927392 .22805-0.00005$

C $7.53565-0.024480$.

H 7.24592 -2.17921 0.00005

H $7.404352 .14876-0.00005$

C -4.69037 -0.079810.

C -5.361581 .156010 .00003$ 
$\begin{array}{llll}\text { C }-5.45076 & -1.26799 & -0.00003 \\ \text { C }-6.761 & 1.20108 & 0.00003 \\ \text { H }-4.78184 & 2.08265 & 0.00005 \\ \text { C }-6.84375 & -1.20912 & -0.00002 \\ \text { H }-4.92739 & -2.22805 & -0.00005 \\ \text { C }-7.53565 & 0.02448 & 0.00001 \\ \text { H }-7.24592 & 2.17921 & 0.00006 \\ \text { H }-7.40435 & -2.14876 & -0.00004 \\ \text { C } 9.07387 & -0.03851 & 0.00001 \\ \text { C } 9.59263 & 0.69062 & -1.26531 \\ \text { C } 9.59261 & 0.69098 & 1.26513 \\ \text { C } 9.64315 & -1.4713 & 0.00021 \\ \text { H } 9.23445 & 0.18853 & -2.1835 \\ \text { H } 9.2557 & 1.74275 & -1.29984 \\ \text { H } 10.69964 & 0.68934 & -1.27977 \\ \text { H } 9.23446 & 0.18912 & 2.18345 \\ \text { H } 10.69962 & 0.68974 & 1.27958 \\ \text { H } 9.25564 & 1.7431 & 1.29938 \\ \text { H } 10.74839 & -1.43064 & 0.00011 \\ \text { H } 9.33006 & -2.03693 & 0.89751 \\ \text { H } 9.32991 & -2.03725 & -0.89683 \\ \text { C }-9.07387 & 0.03851 & 0.00001 \\ \text { C }-9.59263 & -0.69069 & -1.26527 \\ \text { C - } 9.59262 & -0.69091 & 1.26518 \\ \text { C }-9.64315 & 1.4713 & 0.00014 \\ \text { H }-9.23446 & -0.18864 & -2.18348 \\ \text { H - } 9.25568 & -1.74282 & -1.29975 \\ \text { H }-10.69963 & -0.68942 & -1.27972 \\ \text { H }-9.23446 & -0.189 & 2.18347 \\ \text { H - }-10.69962 & -0.68966 & 1.27963 \\ \text { H }-9.25566 & -1.74303 & 1.29948 \\ \text { H }-10.74839 & 1.43064 & 0.0001 \\ \text { H }-9.33002 & 2.037 & 0.89737 \\ \text { H - }-9.32995 & 2.03718 & -0.89696\end{array}$

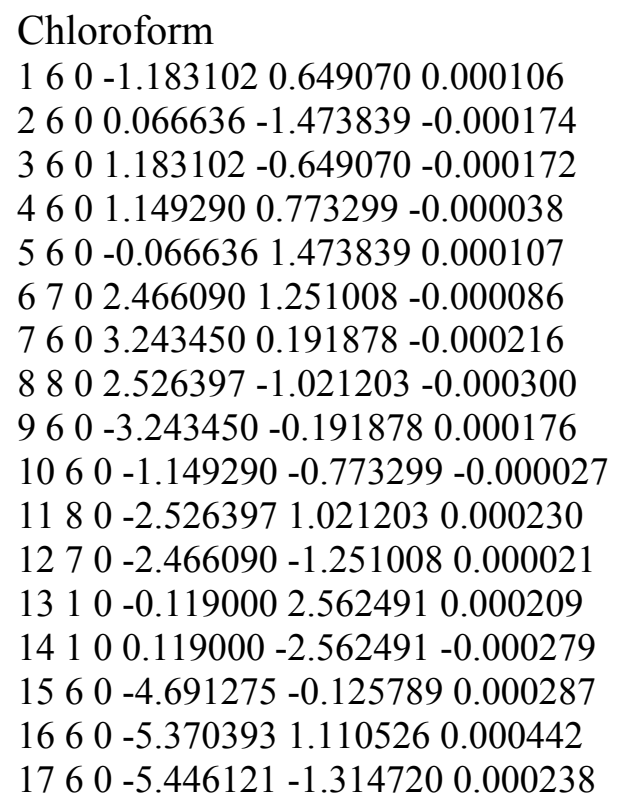


$1860-6.7662581 .1445970 .000548$ $1910-4.7986962 .0420030 .000480$ $2060-6.844025-1.2670270 .000344$ $2110-4.924611-2.2757080 .000117$ $2260-7.538793-0.0392060 .000502$ $2310-7.2618892 .1198240 .000665$ $2410-7.393089-2.2104500 .000302$ $25604.6912750 .125788-0.000305$ $26605.370393-1.110526-0.000476$ $27605.4461211 .314720-0.000219$ $28606.766258-1.144598-0.000555$ $29104.798696-2.042003-0.000546$ $30606.8440251 .267027-0.000297$ $31104.9246112 .275708-0.000086$ $32607.5387930 .039206-0.000466$ $33107.261889-2.119824-0.000687$ $34107.3930892 .210450-0.000221$ $35609.074261-0.048347-0.000554$ $36609.542493-0.8118251 .264444$ $37109.134753-1.8387311 .298798$ $381010.646807-0.8856671 .275689$ $39109.222785-0.2848362 .183116$ $40609.7366201 .343792-0.000371$ 41109.4620511 .9292490 .896892 $421010.8365381 .228877-0.000435$ $43109.4619771 .929520-0.897434$ $44609.542365-0.811429-1.265838$ $45109.134615-1.838321-1.300474$ $46109.222569-0.284148-2.184311$ $471010.646678-0.885274-1.277216$

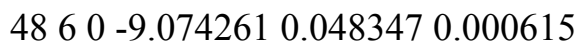
$4960-9.5423470 .8114501 .265893$ $\begin{array}{lllll}50 & 1 & 0 & -9.134611 & 1.8383491 .300499\end{array}$

$\begin{array}{lllllll}51 & 1 & 0 & -10.646661 & 0.885281 & 1.277292\end{array}$ $\begin{array}{llllll}52 & 1 & 0 & -9.222526 & 0.2841932 .184371\end{array}$ $\begin{array}{llllll}53 & 6 & 0 & -9.542510 & 0.811804 & -1.264389\end{array}$

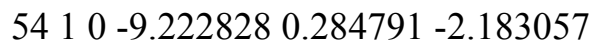
$5510-10.6468240 .885660-1.275613$

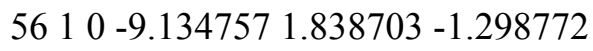
$5760-9.736620-1.3437920 .000465$

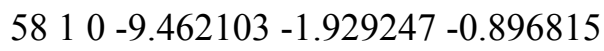
$5910-9.461925-1.9295220 .897511$ $6010-10.836538-1.2288770 .000591$

\section{Energy Levels}

Gas Phase

Alpha occ. eigenvalues -- -19.20690 -19.20690 -14.33070 -14.33069 -10.28387 Alpha occ. eigenvalues -- -10.28387 -10.25293 -10.25286 -10.22697 -10.22691 Alpha occ. eigenvalues -- -10.20258 -10.20258 -10.20161 -10.20161 -10.20137 Alpha occ. eigenvalues -- -10.20128 -10.19653 -10.19653-10.18818 -10.18818 
Alpha occ. eigenvalues -- -10.18657 -10.18657 -10.18276 -10.18276 -10.18239

Alpha occ. eigenvalues -- -10.18239 -10.16465 -10.16465 -10.16463-10.16463

Alpha occ. eigenvalues -- -10.16430 -10.16430 -1.13767 -1.13665 -0.94693

Alpha occ. eigenvalues -- $-0.94621-0.88650-0.87874-0.86957-0.83355$

Alpha occ. eigenvalues -- $-0.82862-0.79044-0.77524-0.76784-0.75750$

Alpha occ. eigenvalues -- $-0.75670-0.73237-0.69144-0.69083-0.68839$

Alpha occ. eigenvalues -- $-0.68839-0.68403-0.65155-0.64349-0.62884$

Alpha occ. eigenvalues -- $-0.62286-0.60837-0.60493-0.58892-0.56172$

Alpha occ. eigenvalues -- $-0.56029-0.56011-0.55528-0.52909-0.49675$

Alpha occ. eigenvalues -- $-0.49673-0.48931-0.48301-0.48259-0.47759$

Alpha occ. eigenvalues -- $-0.47678-0.47376-0.46432-0.45527-0.45432$

Alpha occ. eigenvalues -- $-0.45285-0.45107-0.45102-0.43814-0.43804$

Alpha occ. eigenvalues -- -0.43086 -0.42977 -0.40679 -0.40564 -0.40426

Alpha occ. eigenvalues -- -0.40377 -0.40025 -0.38942 -0.38580 -0.38574

Alpha occ. eigenvalues -- $-0.38209-0.37684-0.37360-0.37343-0.36510$

Alpha occ. eigenvalues -- -0.36341 -0.36257 -0.35967 -0.34593 -0.34328

Alpha occ. eigenvalues -- $-0.33833-0.33821-0.33096-0.32933-0.32922$

Alpha occ. eigenvalues -- $-0.29410-0.29084-0.28995-0.27015-0.26983$

Alpha occ. eigenvalues -- $-0.25852-0.25564-0.22422$

Alpha virt. eigenvalues -- $-0.08083-0.05062-0.01866-0.01784-0.01320$

Alpha virt. eigenvalues -- 0.007440 .043190 .051030 .051130 .05563

Alpha virt. eigenvalues -- 0.080370 .082300 .087560 .089990 .09261

Alpha virt. eigenvalues -- 0.097790 .098670 .103770 .112330 .11238

Alpha virt. eigenvalues -- 0.114170 .115530 .121520 .121630 .12992

Alpha virt. eigenvalues -- 0.131160 .132800 .136160 .143460 .14637

Alpha virt. eigenvalues -- 0.157070 .162790 .163010 .169740 .16987

Alpha virt. eigenvalues -- 0.172840 .173850 .177970 .185360 .18539

Alpha virt. eigenvalues -- 0.185610 .187110 .192780 .196200 .19621

Alpha virt. eigenvalues -- 0.200250 .213220 .219680 .229080 .23821

Alpha virt. eigenvalues -- 0.239900 .242890 .242900 .248550 .27163

Alpha virt. eigenvalues -- 0.276780 .279170 .284830 .284990 .28766

Alpha virt. eigenvalues -- 0.297450 .303430 .313930 .314550 .33159

Alpha virt. eigenvalues -- 0.332660 .337680 .355790 .356680 .36202

Alpha virt. eigenvalues -- 0.369170 .376080 .390190 .398510 .41523

Alpha virt. eigenvalues -- 0.415240 .417970 .420910 .427370 .42937

Alpha virt. eigenvalues -- 0.432590 .436590 .438400 .438550 .45549

Alpha virt. eigenvalues -- 0.455700 .457130 .462760 .470400 .47278

Alpha virt. eigenvalues -- 0.474970 .480480 .482170 .486220 .50083

Alpha virt. eigenvalues -- 0.503630 .507720 .518100 .521190 .53439

Alpha virt. eigenvalues -- 0.535470 .539490 .547420 .549100 .55007

Alpha virt. eigenvalues -- 0.570820 .572970 .575670 .581010 .58257

Alpha virt. eigenvalues -- 0.583770 .584560 .590690 .601830 .61460

Alpha virt. eigenvalues -- 0.615370 .615960 .635910 .635950 .63704

Alpha virt. eigenvalues -- 0.639150 .644630 .645150 .663800 .66579

Alpha virt. eigenvalues -- 0.668910 .669980 .670210 .672340 .67364

Alpha virt. eigenvalues -- 0.676130 .681400 .682600 .686400 .69116

Alpha virt. eigenvalues -- 0.693690 .693830 .694260 .699910 .70337

Alpha virt. eigenvalues -- 0.704320 .706280 .706570 .714200 .71560

Alpha virt. eigenvalues -- 0.716330 .717090 .722730 .729100 .73078

Alpha virt. eigenvalues -- 0.734670 .734810 .734980 .736460 .74010

Alpha virt. eigenvalues -- 0.741920 .742160 .750190 .757240 .75727

Alpha virt. eigenvalues -- 0.757570 .765310 .766890 .773100 .78052

Alpha virt. eigenvalues -- 0.786810 .795550 .795710 .800450 .80389

Alpha virt. eigenvalues -- 0.807870 .821150 .822540 .839460 .84110

Alpha virt. eigenvalues -- 0.841220 .845600 .859910 .869920 .87032

Alpha virt. eigenvalues -- 0.881780 .907400 .909110 .915710 .91724 
Alpha virt. eigenvalues -- 0.924540 .948670 .960290 .968030 .97480 Alpha virt. eigenvalues -- 0.992060 .997460 .999411 .027651 .03013 Alpha virt. eigenvalues -- 1.035591 .049361 .076481 .079121 .08161 Alpha virt. eigenvalues -- 1.100021 .122841 .123461 .124601 .12612 Alpha virt. eigenvalues -- 1.129671 .151421 .188541 .196461 .21550 Alpha virt. eigenvalues -- 1.229891 .263071 .275581 .277311 .28741 Alpha virt. eigenvalues -- 1.288161 .312261 .318351 .351321 .36185 Alpha virt. eigenvalues -- 1.382671 .425121 .448861 .498231 .52077 Alpha virt. eigenvalues -- 1.586131 .589001 .707121 .766111 .80314 Alpha virt. eigenvalues -- 1.82568

\section{Chloroform}

Alpha occ. eigenvalues -- -19.21473 -19.21472 -14.33933 -14.33932 -10.29244 Alpha occ. eigenvalues -- -10.29243 -10.25992 -10.25985 -10.23442 -10.23436 Alpha occ. eigenvalues -- -10.20754 -10.20745 -10.20713 -10.20712 -10.20060 Alpha occ. eigenvalues -- -10.20060 -10.19928 -10.19928 -10.19239 -10.19239 Alpha occ. eigenvalues -- -10.19140 -10.19140 -10.18551-10.18551-10.18459 Alpha occ. eigenvalues -- -10.18459 -10.16155 -10.16155 -10.16153 -10.16153 Alpha occ. eigenvalues -- -10.16005 -10.16005 -1.14536 -1.14434 -0.95419 Alpha occ. eigenvalues -- $-0.95345-0.89228-0.88231-0.87395-0.83441$ Alpha occ. eigenvalues -- $-0.82885-0.79465-0.78154-0.77087-0.76087$ Alpha occ. eigenvalues -- $-0.76011-0.73681-0.68962-0.68798-0.68772$ Alpha occ. eigenvalues -- $-0.68543-0.68543-0.65765-0.64730-0.63499$ Alpha occ. eigenvalues -- -0.62648 -0.61122 -0.60932 -0.59432 -0.56767 Alpha occ. eigenvalues -- -0.56308 -0.56073 -0.55859-0.53222 -0.50431 Alpha occ. eigenvalues -- -0.49827 -0.49481 -0.49029 -0.48730 -0.48044 Alpha occ. eigenvalues -- $-0.47758-0.47750-0.46640-0.45952-0.45705$ Alpha occ. eigenvalues -- -0.45586 -0.44809-0.44803-0.44068 -0.44023 Alpha occ. eigenvalues -- -0.42972 -0.42947 -0.41175 -0.40733-0.40500 Alpha occ. eigenvalues -- -0.40479 -0.40210 -0.39168 -0.38486 -0.38368 Alpha occ. eigenvalues -- -0.38220 -0.38182 -0.37065 -0.37029 -0.36824 Alpha occ. eigenvalues -- -0.36668 -0.36621 -0.36554-0.34706 -0.34135 Alpha occ. eigenvalues -- $-0.33732-0.33727-0.33442-0.32775-0.32768$ Alpha occ. eigenvalues -- $-0.30470-0.30128-0.29479-0.27444-0.27442$ Alpha occ. eigenvalues -- $-0.26281-0.26271-0.23033$

Alpha virt. eigenvalues -- -0.08717 -0.05699 -0.02273-0.02179-0.02008 Alpha virt. eigenvalues -- 0.001570 .037590 .048920 .055660 .05589 Alpha virt. eigenvalues -- 0.078590 .084270 .089340 .090490 .09417 Alpha virt. eigenvalues -- 0.102250 .102660 .103490 .112940 .11521 Alpha virt. eigenvalues -- 0.115800 .115840 .125150 .125710 .12579 Alpha virt. eigenvalues -- 0.126670 .137710 .139610 .139860 .14366 Alpha virt. eigenvalues -- 0.151240 .163410 .163890 .168860 .17256 Alpha virt. eigenvalues -- 0.173720 .173760 .176320 .185290 .18907 Alpha virt. eigenvalues -- 0.189090 .189860 .191520 .193560 .19891 Alpha virt. eigenvalues -- 0.201020 .218570 .219290 .222280 .23576 Alpha virt. eigenvalues -- 0.240080 .244540 .244580 .244590 .26292 Alpha virt. eigenvalues -- 0.272540 .276060 .279100 .280250 .28076 Alpha virt. eigenvalues -- 0.293540 .296800 .308550 .311140 .32695 Alpha virt. eigenvalues -- 0.327420 .337760 .350880 .351850 .36107 Alpha virt. eigenvalues -- 0.363640 .369980 .381780 .395640 .41181 Alpha virt. eigenvalues -- 0.417780 .418450 .418460 .419430 .42861 Alpha virt. eigenvalues -- 0.428690 .431630 .432670 .439450 .44923 Alpha virt. eigenvalues -- 0.453380 .454370 .458170 .466300 .47035 Alpha virt. eigenvalues -- 0.472380 .479600 .479830 .482920 .49538 Alpha virt. eigenvalues -- 0.498250 .504580 .513740 .516900 .52788 
Alpha virt. eigenvalues -- 0.530150 .535080 .542960 .545230 .54825 Alpha virt. eigenvalues -- 0.567260 .569610 .572030 .574510 .57809 Alpha virt. eigenvalues -- 0.580800 .582320 .589340 .599390 .60842 Alpha virt. eigenvalues -- 0.617360 .618690 .632910 .634520 .63457 Alpha virt. eigenvalues -- 0.635580 .645930 .646000 .659850 .66573 Alpha virt. eigenvalues -- 0.669220 .670840 .671040 .671150 .67563 Alpha virt. eigenvalues -- 0.677030 .682720 .682810 .684330 .68812 Alpha virt. eigenvalues -- 0.689990 .695360 .695400 .697800 .70349 Alpha virt. eigenvalues -- 0.705220 .707730 .708060 .713710 .71510 Alpha virt. eigenvalues -- 0.715420 .716010 .717080 .723720 .72854 Alpha virt. eigenvalues -- 0.731480 .733350 .735750 .735920 .73703 Alpha virt. eigenvalues -- 0.737060 .738620 .754890 .757900 .75879 Alpha virt. eigenvalues -- 0.758830 .763210 .768450 .770900 .77362 Alpha virt. eigenvalues -- 0.780090 .793820 .796870 .796930 .80747 Alpha virt. eigenvalues -- 0.811030 .815680 .819030 .833000 .83982 Alpha virt. eigenvalues -- 0.845020 .845110 .855400 .864830 .86509 Alpha virt. eigenvalues -- 0.879560 .900090 .903930 .906170 .90722 Alpha virt. eigenvalues -- 0.909130 .937540 .950540 .967000 .97596 Alpha virt. eigenvalues -- 0.985430 .990821 .013151 .026421 .03342 Alpha virt. eigenvalues -- 1.042391 .044741 .059551 .070111 .07013 Alpha virt. eigenvalues -- 1.099081 .109631 .125001 .125071 .12632 Alpha virt. eigenvalues -- 1.138471 .148071 .179131 .188721 .21274 Alpha virt. eigenvalues -- 1.226751 .264001 .269981 .277011 .29057 Alpha virt. eigenvalues -- 1.291371 .302771 .312851 .330101 .34224 Alpha virt. eigenvalues -- 1.376851 .432251 .444351 .486941 .51767 Alpha virt. eigenvalues -- 1.584671 .587741 .695861 .755831 .79504 Alpha virt. eigenvalues -- 1.81720

\section{Comparison of Experimental and DFT UV-Vis}

Gas Phase

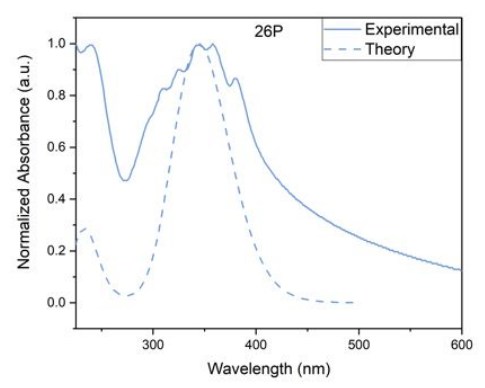

Chloroform

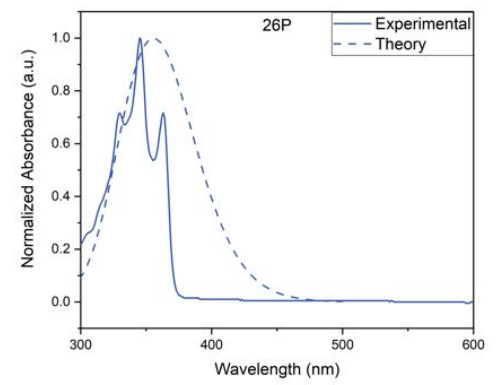

Figure S24. Gas phase and chloroform overlays of computation and experimental data for 26P

\section{Lowest 15 Excited States}

\begin{tabular}{|c|c|c|c|c|c|c|}
\hline \multicolumn{4}{|c|}{ Gas Phase } & \multicolumn{3}{|c|}{ Chloroform } \\
\hline $\begin{array}{l}\text { Excited } \\
\text { state }\end{array}$ & $\frac{\text { Energy }}{(\mathrm{eV})}$ & $\frac{\text { Wavelength }}{(\mathrm{nm})}$ & $f$ & $\frac{\text { Energy }}{(\mathrm{eV})}$ & $\frac{\text { Wavelength }}{(\mathrm{nm})}$ & $f$ \\
\hline 1 & 3.60 & 344.53 & 1.89 & 3.49 & 355.63 & 2.05 \\
\hline 2 & 4.25 & 291.74 & 0.02 & 4.25 & 291.83 & 0.03 \\
\hline 3 & 4.31 & 287.58 & 0.00 & 4.29 & 289.12 & 0.00 \\
\hline 4 & 4.56 & 271.65 & 0.00 & 4.50 & 275.59 & 0.00 \\
\hline 5 & 4.57 & 271.03 & 0.02 & 4.53 & 273.76 & 0.03 \\
\hline
\end{tabular}




\begin{tabular}{|l|lll||lll|}
6 & 4.69 & 264.25 & 0.00 & 4.54 & 273.03 & 0.00 \\
7 & 4.80 & 258.45 & 0.00 & 4.90 & 253.03 & 0.00 \\
8 & 4.88 & 254.31 & 0.00 & 4.98 & 248.91 & 0.00 \\
9 & 5.02 & 246.85 & 0.00 & 5.03 & 346.35 & 0.00 \\
10 & 5.13 & 241.62 & 0.01 & 5.13 & 241.51 & 0.07 \\
11 & 5.15 & 240.52 & 0.00 & 5.16 & 240.29 & 0.00 \\
12 & 5.19 & 238.68 & 0.02 & 5.17 & 239.85 & 0.03 \\
13 & 5.29 & 234.19 & 0.05 & 5.23 & 236.97 & 0.08 \\
14 & 5.31 & 233.41 & 0.45 & 5.25 & 236.37 & 0.38 \\
15 & 5.56 & 222.87 & 0.03 & 5.53 & 224.19 & 0.07 \\
\hline
\end{tabular}

Table S4. Gas phase and chloroform excitation energies, wavelengths, and oscillator strengths for first 15 excited state of $26 \mathrm{P}$.

\section{Geometric Images}

Gas Phase
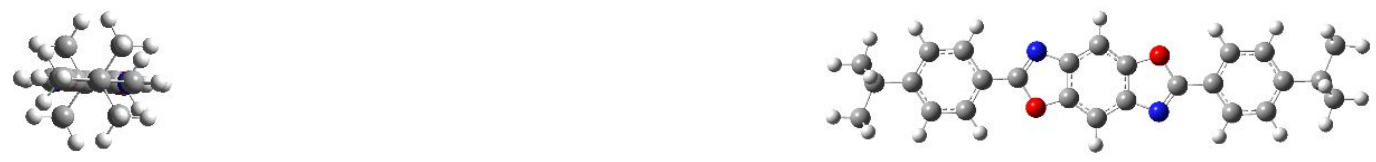

Chloroform
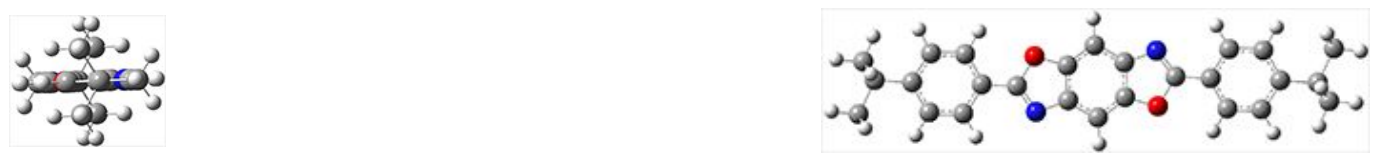

Figure S25. Geometric optimization images in gas phase and chloroform for 26P.

\section{$48 \mathrm{M}$}

\section{Cartesian Coordinates}

\section{Gas Phase}

C - 1.511830 .001450 .00423

C -0.697531 .144060 .00883$

C $1.51183-0.001480 .00391$

C $0.69753-1.144090 .0087$

C $0.721631 .17363-0.00132$

C $0.049763 .24457-0.01093$

O - 1.124212 .476730 .00322

N $1.133092 .52096-0.01229$

C $-0.72163-1.17365-0.00115$

C - $0.04976-3.24459-0.01088$

$\mathrm{N}-1.13309-2.52098-0.01201$

O $1.12421-2.476760 .00301$

C $2.99013-0.024240 .00193$

C $3.726881 .0763-0.48307$

C $3.69469-1.147290 .48497$

C $5.133761 .06714-0.4927$

H $3.190521 .94496-0.86534$

C $5.1011-1.183210 .4877$

H $3.14175-1.994420 .89166$

C $5.88932 .23677-1.01244$

C $5.80705-0.06928-0.00502$ 
C $5.8231-2.3731 .0093$

C $5.420323 .55076-0.82169$

C $7.102372 .07671-1.71476$

H $6.90094-0.08737-0.00731$

C $7.03092-2.24371 .72701$

C $5.32767-3.675220 .80643$

C $6.132984 .65595-1.30795$

H $4.492563 .71597-0.26437$

C $7.808323 .18175-2.19923$

H $7.48691 .07044-1.90876$

C $7.70525-3.366682 .21513$

H $7.43535-1.246921 .92949$

C $6.00844-4.798781 .29634$

H $4.40509-3.816820 .23434$

C $7.344184 .50098-2.00845$

H $5.72735 .65319-1.12318$

H $8.739693 .00516-2.7469$

C $7.21383-4.674312 .01257$

H $8.63332-3.213852 .77543$

H $5.58338-5.785931 .10112$

C -2.990130 .024210 .00258$

C $-3.72699-1.07633-0.48228$

C -3.694581 .147270 .48576$

C $-5.13386-1.06716-0.4916$

H -3.19071 -1.94499-0.86467

C -5.100991 .183190 .48879$

H -3.14154 1.99440 .89233

C $-5.88952-2.23678-1.0112$

C $-5.807040 .06927-0.00377$

C -5.822872 .372991 .01053$

C $-5.42051-3.55078-0.82055$

C $-7.10273-2.0767-1.71326$

H $-6.900940 .08736-0.00583$

C -7.030552 .243711 .72847$

C -5.327473 .67520 .80757$

C $-6.13328-4.65596-1.30668$

$\mathrm{H}-4.49264-3.716-0.26342$

C $-7.80879-3.18173-2.1976$

H - $7.48729-1.07042-1.90719$

C -7.704783 .366692 .21672$

H -7.43496 1.24694 1.93103

C -6.008134 .798781 .2976$

H -4.4053 .816790 .23529$

C -7.34462 -4.50096 -2.00693

H $-5.72758-5.6532-1.12199$

H $-8.74027-3.00512-2.74508$

C -7.213394.67433 2.01405

H -8.63275 3.21388 2.77719

H -5.5831 5.785921 .10229

C $7.98904-5.88162 .56984$

C $8.07923-5.76484 .11243$

C $7.30604-7.219982 .22511$

C $9.41797-5.897921 .97116$

H $8.59641-4.838194 .4223$

H 7.07021 -5.75704 4.5658

H $8.64061-6.62294 .53058$ 
H $7.23302-7.372271 .13206$

H $7.89681-8.057822 .64086$

H $6.28905-7.28282 .65509$

H $9.98988-6.759892 .36619$

H $9.38077-5.9830 .86886$

H $9.97762-4.978062 .22133$

C $8.153145 .68782-2.56157$

C 8.26349 5.55981-4.10196

C $7.494177 .04274-2.23487$

C $9.573275 .67749-1.94217$

H $8.766414 .62119-4.39893$

H 7.26127 $5.56947-4.57011$

H $8.848056 .40397-4.51677$

H $7.408647 .20339-1.14394$

H $8.108777 .86509-2.64708$

H $6.484937 .1246-2.67968$

H $10.169276 .52438-2.33427$

H 9.52198 5.77065 -0.84109

H $10.116454 .7441-2.17831$

C -7.98849 5.88163 2.57145

C -8.078445 .764824 .11405$

C -7.30552 7.22 2.22661

C -9.417515 .897971 .97298$

H -8.59564 .838234 .42399$

H -7.06935 5.75705 4.56727

H -8.63974 6.622944 .53228

H -7.23266 7.37229 1.13356

H -7.8962 8.05785 2.64246

H -6.28845 7.28279 2.65644

H -9.989356 .759952 .3681$

H -9.38047 5.983050 .87067

H -9.977144 .978112 .22324$

C $-8.1537-5.68779-2.55991$

C $-8.26432-5.55978-4.10028$

C $-7.4947-7.04272-2.23332$

C $-9.57372-5.67744-1.94027$

H -8.76727 -4.62115 -4.39717

H - $7.26217-5.56946-4.5686$

H $-8.84896-6.40393-4.51499$

H - $7.40898-7.20337-1.14241$

H $-8.10938-7.86506-2.64542$

H $-6.48554-7.1246-2.67831$

H - $10.16981-6.52432-2.33227$

H -9.52225 -5.77061 -0.8392

H - $10.11693-4.74405-2.17631$

C - $0.107744 .72081-0.01824$

C $1.22635 .46821-0.0263$

H -0.713895 .009210 .86412$

H - $0.720475 .00021-0.89889$

H $1.054596 .55911-0.02696$

H 1.831415 .210470 .86085

H 1.822785 .20887 -0.91891

C $0.10773-4.72084-0.0182$

C $-1.22631-5.46823-0.02607$

H $0.71401-5.009240 .86408$

H $0.72035-5.00024-0.89893$ 
H - $1.05459-6.55914-0.02674$

$\mathrm{H}-1.83129-5.210490 .86117$

H -1.82291 -5.2089-0.91859

\section{Chloroform}

C 0.714551 .134770 .07238

C $-0.71456-1.134780 .07226$

C $0.70303-1.18640 .08243$

N $1.09429-2.53910 .08837$

C $-0.00204-3.24880 .08932$

O - $1.16295-2.458160 .07789$

C 0.002043 .248780 .08964

C - 0.703031 .186390 .08254

O 1.162952 .458140 .07815

$\mathrm{N}-1.094292 .539090 .08861$

C $-0.17555-4.721530 .08578$

H -0.73974 -5.013690.9928

H $0.8309-5.163210 .17152$

C $-0.89939-5.25704-1.16262$

H $-0.9896-6.35613-1.10251$

H -1.91675 -4.83492 -1.24886

H - $0.3427-5.0084-2.08414$

C 0.175554 .721520 .08625

H 0.739755 .013590 .99329

H -0.83095 .163190 .17205$

C $0.899385 .25716-1.16211$

H $0.989596 .35624-1.10188$

H $1.916744 .83504-1.2484$

H $0.342675 .00861-2.08364$

C -1.506980 .021730 .07713$

C $1.50698-0.021740 .07715$

C -2.98540 .022860 .07067$

C -3.705091 .091260 .64368$

C -3.70311 -1.04243 -0.51238

C -5.112881 .113590 .63443$

H -3.15412 1.907121 .11229

C -5.11086-1.04931-0.53306

H -3.15803 -1.86153-0.98404

C -5.800770 .036220 .04158$

C -5.851312 .251911 .24264$

C -5.85019 -2.17886-1.15607

$\mathrm{H}-6.894470 .041010 .0267$

C -5.3233 .557921 .22159$

C - 7.106232 .070951 .86377

C -5.37335 -3.50123-1.06481

C - 7.05665 -1.97138 -1.85908

C -6.015374 .634091 .7954$

$\mathrm{H}-4.363463 .747030 .72998$

C -7.793643.14768 2.43345

$\mathrm{H}-7.544211 .069861 .92583$

C $-6.07027-4.56998-1.64755$

$\mathrm{H}-4.45382-3.70791-0.50776$

C -7.74717 -3.03993 - 2.44027

$\mathrm{H}-7.45057-0.95682-1.97533$

C -7.26745 4.45809 2.41586 
H -5.56035 5.62551 .74287

H -8.76032 .953232 .90873$

C $-7.27451-4.3677-2.3493$

H $-5.65836-5.57542-1.53692$

H -8.67328 -2.82591 -2.98311

C -8.05875 .615823 .05004$

C $-8.06725-5.5137-3.00274$

C -9.412385 .773092 .31206$

C -7.298566 .954452 .96183$

C -8.322485 .304384 .54478$

C $-8.1538-5.27024-4.53085$

C -7.40504 -6.88583 -2.76676

C -9.49737 -5.55464 -2.40773

H - 10.020074 .851582 .37447

H -9.99896 .598022 .76076$

H -9.251696 .005531 .24235$

H -7.11241 7.25254 1.9132

H -7.89925 7.75391 3.43464

H -6.32704 6.908843 .48871

H -8.90995 4.37674 4.67303

H -7.37045 5.18633 5.09599

H -8.89152 6.13151 5.01144

H -8.72841 -6.08349 -5.01484

H -8.65707 -4.31407 -4.76499

H - 7.14426 -5.2446 -4.98298

H - $7.33521-7.12655-1.68948$

H -8.00944 -7.67708 -3.24866

H -6.38877 -6.93029 -3.20071

H - $10.07993-6.3744-2.87064$

H -9.4637 -5.72899-1.31567

H - 10.04319 -4.60996 -2.58617

C $2.98539-0.022870 .07071$

C $3.70508-1.091310 .64365$

C $3.703111 .04246-0.51225$

C $5.11287-1.113640 .63442$

H $3.1541-1.90721 .11219$

C $5.110861 .04934-0.53292$

H $3.158041 .8616-0.98386$

C $5.80077-0.036230 .04166$

C $5.85129-2.2521 .24256$

C $5.85022 .17894-1.15583$

H $6.89446-0.041010 .0268$

C $5.32299-3.558011 .22141$

C $7.1062-2.071081 .86373$

C $5.373363 .5013-1.06449$

C $7.056681 .97151-1.85884$

C $6.01535-4.634221 .79516$

H $4.36346-3.747090 .72978$

C $7.7936-3.147852 .43334$

H $7.54418-1.069991 .92585$

C $6.07034 .57009-1.64714$

H $4.453833 .70794-0.50743$

C $7.74723 .0401-2.43994$

H $7.450590 .95696-1.97516$

C $7.26741-4.458262 .41565$

H 5.56033 -5.62563 1.74257 
H $8.76026-2.953432 .90865$

C $7.274544 .36786-2.34888$

H $5.658395 .57553-1.53644$

H $8.673322 .82612-2.98278$

C $8.05866-5.616033 .04977$

C $8.06735 .51391-3.00222$

C $9.41235-5.773252 .3118$

C $7.29852-6.954652 .96147$

C $8.32242-5.304694 .54453$

C $8.153885 .27056-4.53035$

C $7.405086 .88602-2.76616$

C $9.497415 .55481-2.40718$

H $10.02004-4.851742 .37427$

H $9.99886-6.598212 .76046$

H $9.25167-6.005621 .24207$

H 7.11239-7.25268 1.91282

H $7.8992-7.754143 .43424$

H $6.32699-6.909073 .48833$

H $8.90989-4.377054 .67285$

H 7.37039 -5.18667 5.09574

H $8.89145-6.131845 .01115$

H $8.728516 .08384-5.01426$

H $8.657164 .3144-4.76454$

H 7.14436 5.24495 -4.98251

H $7.335237 .12667-1.68886$

H $8.009497 .67731-3.248$

H $6.388826 .93051-3.20014$

H $10.079976 .3746-2.87001$

H 9.46371 $5.72908-1.3151$

H $10.043234 .61014-2.58566$

Energy Levels

Gas Phase

Alpha occ. eigenvalues -- -19.21027 -19.21026 -14.33540 -14.33540 -10.28688

Alpha occ. eigenvalues -- -10.28687 -10.25399 -10.25388 -10.22893 -10.22883

Alpha occ. eigenvalues -- -10.21510 -10.21499 -10.20126 -10.20126 -10.19530

Alpha occ. eigenvalues -- -10.19530 -10.19436 -10.19423 -10.19409 -10.19409

Alpha occ. eigenvalues -- -10.18552 -10.18552 -10.18461 -10.18461 -10.18385

Alpha occ. eigenvalues -- - $10.18385-10.18266-10.18266-10.18200-10.18200$

Alpha occ. eigenvalues -- -10.18107 -10.18107 -10.17382 -10.17382 -10.17377

Alpha occ. eigenvalues -- -10.17377 -10.17308 -10.17308 -10.17244 -10.17244

Alpha occ. eigenvalues -- -10.17231 -10.17231 -10.17226 -10.17226 -10.17200

Alpha occ. eigenvalues -- -10.17200 -10.17192 -10.17192 -10.17090 -10.17090

Alpha occ. eigenvalues -- -10.17057 -10.17057 -10.16988 -10.16988 -10.16880

Alpha occ. eigenvalues -- -10.16880 -10.15898 -10.15898 -10.15867-10.15866

Alpha occ. eigenvalues -- -10.15865 -10.15865 -10.15778 -10.15778 -10.15766

Alpha occ. eigenvalues -- -10.15766 -10.15761 -10.15761 -1.14210 -1.14104

Alpha occ. eigenvalues -- -0.95035 -0.95016 -0.89103 -0.87718 -0.87297

Alpha occ. eigenvalues -- $-0.86496-0.86492-0.85619-0.85230-0.82266$

Alpha occ. eigenvalues -- $-0.82250-0.82129-0.82047-0.81558-0.80167$

Alpha occ. eigenvalues -- $-0.80126-0.77452-0.77447-0.76885-0.74898$

Alpha occ. eigenvalues -- $-0.74542-0.74507-0.74345-0.74327-0.74101$

Alpha occ. eigenvalues -- $-0.72553-0.71616-0.71223-0.71135-0.69424$

Alpha occ. eigenvalues -- $-0.68470-0.68462-0.68344-0.68340-0.68232$

Alpha occ. eigenvalues -- $-0.68232-0.68127-0.68127-0.65598-0.65569$

Alpha occ. eigenvalues -- -0.64802 -0.63648 -0.63157 -0.62817 -0.62425 
Alpha occ. eigenvalues -- -0.60947 -0.60864 -0.60399 -0.60378 -0.59972 Alpha occ. eigenvalues -- $-0.59387-0.58697-0.57673-0.57587-0.57129$ Alpha occ. eigenvalues -- $-0.56288-0.55203-0.55202-0.55039-0.55038$ Alpha occ. eigenvalues -- $-0.53618-0.53327-0.52865-0.51026-0.50236$ Alpha occ. eigenvalues -- -0.49896 -0.49739 -0.49690 -0.49072 -0.49040 Alpha occ. eigenvalues -- $-0.48550-0.48433-0.47522-0.47343-0.46770$ Alpha occ. eigenvalues -- $-0.46770-0.46237-0.46139-0.45807-0.45227$ Alpha occ. eigenvalues -- $-0.45193-0.45087-0.45084-0.44709-0.44678$ Alpha occ. eigenvalues -- -0.44668 -0.44487 -0.44486 -0.44373 -0.44373 Alpha occ. eigenvalues -- $-0.44239-0.44225-0.44130-0.43967-0.42860$ Alpha occ. eigenvalues -- $-0.42811-0.42646-0.42616-0.42207-0.42190$ Alpha occ. eigenvalues -- $-0.42024-0.42022-0.41219-0.40123-0.40054$ Alpha occ. eigenvalues -- -0.39899 -0.39898 -0.39830 -0.39769-0.39734 Alpha occ. eigenvalues -- $-0.39388-0.39353-0.39187-0.39171-0.38828$ Alpha occ. eigenvalues -- $-0.38786-0.38121-0.38034-0.38023-0.37919$ Alpha occ. eigenvalues -- $-0.37875-0.37832-0.37480-0.37039-0.36983$ Alpha occ. eigenvalues -- $-0.36959-0.36774-0.36760-0.36658-0.36639$ Alpha occ. eigenvalues -- $-0.36525-0.36365-0.36169-0.36121-0.35764$ Alpha occ. eigenvalues -- -0.35732 -0.35214 -0.35175 -0.34728 -0.34716 Alpha occ. eigenvalues -- -0.34481 -0.34308 -0.33648 -0.33630 -0.33441 Alpha occ. eigenvalues -- $-0.33394-0.32897-0.32894-0.32695-0.32549$ Alpha occ. eigenvalues -- -0.32511 -0.32104 -0.32086 -0.31940 -0.31936 Alpha occ. eigenvalues -- $-0.29696-0.29351-0.29172-0.28307-0.28258$ Alpha occ. eigenvalues -- $-0.27054-0.25828-0.25821-0.25643-0.25636$ Alpha occ. eigenvalues -- $-0.25295-0.24258-0.23412-0.22252-0.22231$ Alpha occ. eigenvalues -- -0.21569

Alpha virt. eigenvalues -- $-0.07479-0.03809-0.03725-0.03036-0.02805$ Alpha virt. eigenvalues -- $-0.01197-0.00953-0.00930-0.00804-0.00719$ Alpha virt. eigenvalues -- -0.005400 .014980 .025010 .032640 .03335$ Alpha virt. eigenvalues -- 0.049940 .052000 .054310 .055550 .05654 Alpha virt. eigenvalues -- 0.057160 .057760 .071830 .072400 .08513 Alpha virt. eigenvalues -- 0.085680 .088800 .089380 .092410 .09413 Alpha virt. eigenvalues -- 0.098830 .101040 .102190 .102680 .10369 Alpha virt. eigenvalues -- 0.104610 .108610 .109430 .110680 .11125 Alpha virt. eigenvalues -- 0.114340 .114870 .115810 .116720 .11767 Alpha virt. eigenvalues -- 0.117810 .118990 .124370 .124800 .12598 Alpha virt. eigenvalues -- 0.126270 .127630 .129380 .130040 .13185 Alpha virt. eigenvalues -- 0.133890 .134160 .135660 .140660 .14172 Alpha virt. eigenvalues -- 0.143130 .145830 .148070 .150060 .15722 Alpha virt. eigenvalues -- 0.157890 .160380 .162130 .162940 .16685 Alpha virt. eigenvalues -- 0.166920 .170030 .173370 .173490 .17438 Alpha virt. eigenvalues -- 0.174680 .175660 .176160 .179220 .17944 Alpha virt. eigenvalues -- 0.180720 .182340 .183060 .185410 .18858 Alpha virt. eigenvalues -- 0.190580 .190900 .191450 .191630 .19203 Alpha virt. eigenvalues -- 0.192500 .195850 .195880 .198540 .20251 Alpha virt. eigenvalues -- 0.203860 .204260 .205600 .209850 .21046 Alpha virt. eigenvalues -- 0.215120 .220780 .222920 .224550 .22667 Alpha virt. eigenvalues -- 0.229320 .235410 .241150 .243260 .24344 Alpha virt. eigenvalues -- 0.245130 .246620 .249310 .249460 .25042 Alpha virt. eigenvalues -- 0.251040 .251210 .255390 .266860 .26719 Alpha virt. eigenvalues -- 0.274240 .276020 .276530 .278060 .28052 Alpha virt. eigenvalues -- 0.281990 .293640 .294050 .298840 .29917 Alpha virt. eigenvalues -- 0.302570 .302750 .304790 .311240 .31175 Alpha virt. eigenvalues -- 0.315920 .316920 .319320 .319650 .32393 Alpha virt. eigenvalues -- 0.327250 .329500 .329570 .337480 .33881 Alpha virt. eigenvalues -- 0.341240 .341580 .343950 .346820 .36164 
Alpha virt. eigenvalues -- 0.361780 .364760 .365500 .375500 .37755 Alpha virt. eigenvalues -- 0.377800 .381130 .382960 .386150 .39278 Alpha virt. eigenvalues -- 0.396760 .398380 .401560 .407300 .40950 Alpha virt. eigenvalues -- 0.412250 .420800 .420970 .421160 .42201 Alpha virt. eigenvalues -- 0.422050 .432320 .434020 .435960 .43645 Alpha virt. eigenvalues -- 0.439340 .441170 .441940 .442350 .44251 Alpha virt. eigenvalues -- 0.445070 .446240 .446810 .447400 .45279 Alpha virt. eigenvalues -- 0.456950 .459040 .461600 .462330 .46573 Alpha virt. eigenvalues -- 0.467320 .468400 .470320 .470590 .47333 Alpha virt. eigenvalues -- 0.473590 .479430 .480400 .480830 .48150 Alpha virt. eigenvalues -- 0.481890 .484170 .487080 .488810 .48968 Alpha virt. eigenvalues -- 0.493210 .496530 .498700 .499460 .50507 Alpha virt. eigenvalues -- 0.508890 .509400 .510480 .511810 .51182 Alpha virt. eigenvalues -- 0.514570 .515930 .522010 .524280 .52446 Alpha virt. eigenvalues -- 0.531900 .535090 .538460 .546550 .55049 Alpha virt. eigenvalues -- 0.553160 .559500 .561620 .563150 .56475 Alpha virt. eigenvalues -- 0.568920 .569310 .571690 .575960 .57619 Alpha virt. eigenvalues -- 0.576930 .579330 .580680 .585230 .58544 Alpha virt. eigenvalues -- 0.597430 .597760 .599270 .599950 .60245 Alpha virt. eigenvalues -- 0.602710 .606450 .610260 .614600 .61813 Alpha virt. eigenvalues -- 0.621400 .621660 .623300 .623620 .62385 Alpha virt. eigenvalues -- 0.626580 .626670 .629070 .629130 .63283 Alpha virt. eigenvalues -- 0.635720 .636020 .637070 .640430 .64606 Alpha virt. eigenvalues -- 0.647610 .651880 .651900 .653550 .65418 Alpha virt. eigenvalues -- 0.660750 .662720 .665540 .666140 .66910 Alpha virt. eigenvalues -- 0.670890 .671560 .672120 .672690 .67366 Alpha virt. eigenvalues -- 0.675610 .675750 .676040 .676210 .67726 Alpha virt. eigenvalues -- 0.678080 .680640 .682190 .683050 .68412 Alpha virt. eigenvalues -- 0.686280 .686350 .688550 .689360 .69036 Alpha virt. eigenvalues -- 0.691020 .692850 .693290 .694210 .69458 Alpha virt. eigenvalues -- 0.695810 .696540 .698270 .699500 .70003 Alpha virt. eigenvalues -- 0.700680 .701920 .703400 .703560 .70533 Alpha virt. eigenvalues -- 0.706680 .706680 .709710 .710320 .71420 Alpha virt. eigenvalues -- 0.714930 .715980 .718520 .720540 .72137 Alpha virt. eigenvalues -- 0.721680 .722990 .724890 .725250 .72624 Alpha virt. eigenvalues -- 0.727100 .728000 .729060 .729480 .73141 Alpha virt. eigenvalues -- 0.731470 .733300 .735830 .735920 .73726 Alpha virt. eigenvalues -- 0.741300 .742120 .743040 .744240 .74623 Alpha virt. eigenvalues -- 0.749070 .750820 .751340 .751520 .75424 Alpha virt. eigenvalues -- 0.755530 .756110 .757510 .759040 .76180 Alpha virt. eigenvalues -- 0.763630 .764260 .765760 .767130 .76851 Alpha virt. eigenvalues -- 0.771270 .771510 .774050 .775400 .77810 Alpha virt. eigenvalues -- 0.778710 .784370 .784880 .786940 .78797 Alpha virt. eigenvalues -- 0.790380 .794740 .794850 .799710 .79990 Alpha virt. eigenvalues -- 0.802460 .804130 .804360 .807090 .81196 Alpha virt. eigenvalues -- 0.816890 .817770 .818030 .820420 .82164 Alpha virt. eigenvalues -- 0.826740 .827250 .833810 .836310 .84074 Alpha virt. eigenvalues -- 0.842650 .844950 .845530 .846330 .84731 Alpha virt. eigenvalues -- 0.850690 .850810 .853810 .855170 .85675 Alpha virt. eigenvalues -- 0.857750 .865840 .867700 .869180 .87171 Alpha virt. eigenvalues -- 0.876930 .882550 .882870 .887190 .89079 Alpha virt. eigenvalues -- 0.895550 .900470 .906010 .912020 .91426 Alpha virt. eigenvalues -- 0.917150 .918080 .930130 .941330 .94702 Alpha virt. eigenvalues -- 0.947940 .968920 .989080 .990610 .99063 Alpha virt. eigenvalues -- 1.014231 .018201 .021231 .025401 .02896 Alpha virt. eigenvalues -- 1.030751 .035271 .036871 .045931 .04720 
Alpha virt. eigenvalues -- 1.050031 .055151 .059051 .065241 .06899 Alpha virt. eigenvalues -- 1.082801 .083161 .088851 .093651 .09458 Alpha virt. eigenvalues -- 1.096371 .102501 .105971 .110941 .11417 Alpha virt. eigenvalues -- 1.118071 .123351 .123551 .125671 .13326 Alpha virt. eigenvalues -- 1.136731 .137771 .138491 .140301 .14467 Alpha virt. eigenvalues -- 1.150151 .153271 .168121 .169091 .16991 Alpha virt. eigenvalues -- 1.179961 .183581 .185651 .200161 .20813 Alpha virt. eigenvalues -- 1.211491 .229231 .237421 .240061 .24676 Alpha virt. eigenvalues -- 1.251551 .264331 .271491 .275541 .28678 Alpha virt. eigenvalues -- 1.293731 .294961 .319051 .319531 .32331 Alpha virt. eigenvalues -- 1.326751 .337621 .350191 .356231 .36437 Alpha virt. eigenvalues -- 1.371531 .377281 .389731 .408321 .40920 Alpha virt. eigenvalues -- 1.433491 .440281 .445161 .462841 .47578 Alpha virt. eigenvalues -- 1.485421 .519311 .561591 .591601 .59475 Alpha virt. eigenvalues -- 1.598221 .598661 .611641 .728451 .77292 Alpha virt. eigenvalues -- 1.774371 .85038

\section{Chloroform}

Alpha occ. eigenvalues -- -19.21527 -19.21526 -14.34056 -14.34056 -10.29091 Alpha occ. eigenvalues -- -10.29090 - 10.25985 -10.25974 -10.23469 -10.23460 Alpha occ. eigenvalues -- -10.22170 -10.22159-10.20422 -10.20409-10.19620 Alpha occ. eigenvalues -- -10.19620 -10.19593 -10.19593-10.19578 -10.19578 Alpha occ. eigenvalues -- -10.19566 -10.19566 -10.19534 -10.19534-10.19150 Alpha occ. eigenvalues -- -10.19150 -10.19070 -10.19070 -10.18809-10.18809 Alpha occ. eigenvalues -- -10.18733 -10.18733 -10.18290 -10.18290 -10.18224 Alpha occ. eigenvalues -- -10.18223 -10.18010 -10.18010 - 10.17992 -10.17991 Alpha occ. eigenvalues -- -10.17970 -10.17970 -10.17939-10.17939 -10.17820 Alpha occ. eigenvalues -- $-10.17820-10.17759-10.17759-10.17697-10.17697$ Alpha occ. eigenvalues -- -10.17685 -10.17685 -10.17586 -10.17586 -10.17245 Alpha occ. eigenvalues -- -10.17245 -10.15857 -10.15857 -10.15849-10.15849 Alpha occ. eigenvalues -- -10.15815 -10.15815 -10.15805-10.15805 -10.15754 Alpha occ. eigenvalues -- -10.15754 -10.15701 -10.15701 -1.14672 -1.14564 Alpha occ. eigenvalues -- -0.95437 -0.95427 -0.89797 -0.88598 -0.88042 Alpha occ. eigenvalues -- $-0.87110-0.87109-0.86281-0.85892-0.82608$ Alpha occ. eigenvalues -- $-0.82599-0.82579-0.82137-0.81900-0.80719$ Alpha occ. eigenvalues -- $-0.80085-0.78127-0.78124-0.77627-0.75331$ Alpha occ. eigenvalues -- $-0.75065-0.75034-0.74978-0.74909-0.74647$ Alpha occ. eigenvalues -- $-0.73180-0.72362-0.71942-0.71861-0.69074$ Alpha occ. eigenvalues -- -0.68437 -0.68431 -0.68380 -0.68377 -0.68234 Alpha occ. eigenvalues -- -0.68234 -0.68189 -0.68189-0.66402 -0.65729 Alpha occ. eigenvalues -- $-0.65253-0.64339-0.63875-0.63754-0.63018$ Alpha occ. eigenvalues -- $-0.61597-0.61013-0.60943-0.60876-0.60503$ Alpha occ. eigenvalues -- $-0.60415-0.59237-0.58548-0.58291-0.57734$ Alpha occ. eigenvalues -- $-0.56596-0.55531-0.55515-0.55437-0.55433$ Alpha occ. eigenvalues -- -0.54242 -0.53463 - $0.53450-0.51107-0.50229$ Alpha occ. eigenvalues -- $-0.50125-0.50011-0.49911-0.49784-0.49323$ Alpha occ. eigenvalues -- $-0.49020-0.48867-0.47914-0.47723-0.47216$ Alpha occ. eigenvalues -- $-0.47205-0.47196-0.46054-0.45810-0.45574$ Alpha occ. eigenvalues -- $-0.45556-0.45470-0.45415-0.45331-0.45181$ Alpha occ. eigenvalues -- $-0.45158-0.44768-0.44734-0.44640-0.44507$ Alpha occ. eigenvalues -- -0.44463 -0.44456 -0.44396 -0.44370 -0.43184 Alpha occ. eigenvalues -- -0.43137-0.43070 -0.43064 -0.42501 -0.42495 Alpha occ. eigenvalues -- $-0.42420-0.42405-0.41913-0.41240-0.40554$ Alpha occ. eigenvalues -- $-0.40504-0.40068-0.40067-0.39979-0.39971$ Alpha occ. eigenvalues -- $-0.39850-0.39849-0.39588-0.39536-0.39463$ 
Alpha occ. eigenvalues -- -0.39225 -0.38039 -0.38033 -0.37950 -0.37935 Alpha occ. eigenvalues -- $-0.37898-0.37717-0.37574-0.37528-0.37429$ Alpha occ. eigenvalues -- $-0.37202-0.37076-0.36771-0.36751-0.36735$ Alpha occ. eigenvalues -- $-0.36670-0.36649-0.36397-0.36374-0.36306$ Alpha occ. eigenvalues -- $-0.36223-0.35766-0.35757-0.35208-0.35201$ Alpha occ. eigenvalues -- $-0.35073-0.34893-0.33764-0.33760-0.33619$ Alpha occ. eigenvalues -- $-0.33568-0.33115-0.33115-0.33088-0.32896$ Alpha occ. eigenvalues -- $-0.32856-0.32264-0.32254-0.32176-0.32173$ Alpha occ. eigenvalues -- -0.30446 -0.30037 -0.29939-0.29225 -0.29139 Alpha occ. eigenvalues -- $-0.27928-0.26511-0.26504-0.26418-0.26410$ Alpha occ. eigenvalues -- $-0.25858-0.24952-0.24171-0.23099-0.23074$ Alpha occ. eigenvalues -- -0.22480

Alpha virt. eigenvalues -- $-0.08113-0.04782-0.04715-0.03776-0.03725$ Alpha virt. eigenvalues -- $-0.01945-0.01637-0.01597-0.01527-0.01461$ Alpha virt. eigenvalues -- -0.011350 .006770 .017060 .024970 .02561$ Alpha virt. eigenvalues -- 0.044940 .051150 .052370 .057660 .05789 Alpha virt. eigenvalues -- 0.058790 .059620 .074450 .075720 .08463 Alpha virt. eigenvalues -- 0.085520 .089680 .090610 .094500 .09894 Alpha virt. eigenvalues -- 0.101750 .102540 .103420 .103890 .10502 Alpha virt. eigenvalues -- 0.105160 .108750 .108960 .110200 .11066 Alpha virt. eigenvalues -- 0.113450 .113830 .115370 .116100 .11768 Alpha virt. eigenvalues -- 0.117800 .118560 .120370 .122880 .12377 Alpha virt. eigenvalues -- 0.127050 .127140 .128690 .129190 .13141 Alpha virt. eigenvalues -- 0.131800 .134980 .136870 .137800 .13837 Alpha virt. eigenvalues -- 0.139320 .143230 .143750 .147830 .15490 Alpha virt. eigenvalues -- 0.154940 .159920 .162050 .163250 .16436 Alpha virt. eigenvalues -- 0.164690 .168290 .171660 .174890 .17522 Alpha virt. eigenvalues -- 0.175450 .176200 .176650 .177630 .17936 Alpha virt. eigenvalues -- 0.179890 .182360 .184060 .187600 .18877 Alpha virt. eigenvalues -- 0.189050 .191660 .191930 .192040 .19244 Alpha virt. eigenvalues -- 0.193590 .194150 .196500 .196950 .20015 Alpha virt. eigenvalues -- 0.203610 .205290 .206500 .209360 .21091 Alpha virt. eigenvalues -- 0.215250 .219540 .222340 .223520 .22507 Alpha virt. eigenvalues -- 0.230620 .231670 .238510 .239970 .24055 Alpha virt. eigenvalues -- 0.242370 .243910 .248030 .248170 .24854 Alpha virt. eigenvalues -- 0.249470 .255860 .258450 .262770 .26299 Alpha virt. eigenvalues -- 0.268150 .268750 .270160 .270430 .27133 Alpha virt. eigenvalues -- 0.273130 .283740 .286510 .290920 .29395 Alpha virt. eigenvalues -- 0.295170 .298140 .298390 .304790 .30523 Alpha virt. eigenvalues -- 0.308770 .310410 .311610 .313480 .31458 Alpha virt. eigenvalues -- 0.321320 .324100 .325380 .330130 .33122 Alpha virt. eigenvalues -- 0.333260 .333610 .334330 .338080 .35516 Alpha virt. eigenvalues -- 0.356020 .357420 .357820 .365290 .36588 Alpha virt. eigenvalues -- 0.369130 .373190 .376170 .383590 .38836 Alpha virt. eigenvalues -- 0.389860 .391530 .392880 .398010 .40382 Alpha virt. eigenvalues -- 0.404730 .408170 .418080 .421100 .42110 Alpha virt. eigenvalues -- 0.421520 .421520 .424370 .431310 .43302 Alpha virt. eigenvalues -- 0.435870 .436050 .436850 .437900 .44134 Alpha virt. eigenvalues -- 0.442060 .442180 .443400 .447310 .45160 Alpha virt. eigenvalues -- 0.452180 .455540 .457410 .458200 .45934 Alpha virt. eigenvalues -- 0.459450 .463030 .463630 .465870 .46845 Alpha virt. eigenvalues -- 0.468520 .472640 .474180 .474580 .47565 Alpha virt. eigenvalues -- 0.480680 .481390 .482020 .482810 .48555 Alpha virt. eigenvalues -- 0.487180 .488350 .492140 .492710 .49344 Alpha virt. eigenvalues -- 0.498820 .501100 .503290 .503880 .50536 Alpha virt. eigenvalues -- 0.507930 .511390 .514810 .515390 .51644 
Alpha virt. eigenvalues -- 0.520720 .523520 .532260 .541030 .54175 Alpha virt. eigenvalues -- 0.545300 .550140 .555440 .556410 .56076 Alpha virt. eigenvalues -- 0.561190 .565560 .566500 .570060 .57237 Alpha virt. eigenvalues -- 0.574260 .577550 .579500 .581060 .58119 Alpha virt. eigenvalues -- 0.587800 .588340 .592110 .593590 .59881 Alpha virt. eigenvalues -- 0.599350 .603210 .604260 .606600 .60964 Alpha virt. eigenvalues -- 0.615730 .617930 .620160 .620240 .62055 Alpha virt. eigenvalues -- 0.620890 .621790 .622830 .624620 .63004 Alpha virt. eigenvalues -- 0.631310 .632540 .634240 .634690 .64090 Alpha virt. eigenvalues -- 0.648500 .649550 .649570 .650570 .65123 Alpha virt. eigenvalues -- 0.655390 .656600 .661620 .666500 .66803 Alpha virt. eigenvalues -- 0.670830 .671240 .671750 .672770 .67349 Alpha virt. eigenvalues -- 0.673950 .674200 .674600 .675260 .67554 Alpha virt. eigenvalues -- 0.677120 .677270 .678900 .681130 .68147 Alpha virt. eigenvalues -- 0.683080 .683480 .685740 .687020 .68820 Alpha virt. eigenvalues -- 0.689500 .690550 .691410 .691920 .69237 Alpha virt. eigenvalues -- 0.693800 .695110 .696380 .698100 .69842 Alpha virt. eigenvalues -- 0.700530 .700820 .702290 .703870 .70434 Alpha virt. eigenvalues -- 0.704930 .706640 .709600 .710590 .71297 Alpha virt. eigenvalues -- 0.713870 .714870 .715710 .717640 .72085 Alpha virt. eigenvalues -- 0.723040 .723290 .724520 .724650 .72552 Alpha virt. eigenvalues -- 0.726940 .727200 .727720 .728870 .73095 Alpha virt. eigenvalues -- 0.732210 .732800 .733430 .734720 .73578 Alpha virt. eigenvalues -- 0.738500 .738850 .739850 .742570 .74408 Alpha virt. eigenvalues -- 0.747160 .747790 .749630 .750710 .75392 Alpha virt. eigenvalues -- 0.754260 .756340 .756510 .758580 .76075 Alpha virt. eigenvalues -- 0.761640 .762080 .763340 .765330 .76564 Alpha virt. eigenvalues -- 0.766480 .771740 .775380 .775880 .77648 Alpha virt. eigenvalues -- 0.777270 .781140 .782810 .784630 .78942 Alpha virt. eigenvalues -- 0.789580 .794420 .798100 .798340 .79935 Alpha virt. eigenvalues -- 0.800690 .805850 .807590 .811410 .81251 Alpha virt. eigenvalues -- 0.818200 .818970 .818980 .820840 .82364 Alpha virt. eigenvalues -- 0.831570 .835000 .836630 .838300 .83923 Alpha virt. eigenvalues -- 0.843100 .843960 .846250 .846780 .84704 Alpha virt. eigenvalues -- 0.847270 .847750 .848690 .851630 .85433 Alpha virt. eigenvalues -- 0.856970 .859030 .863890 .865940 .86862 Alpha virt. eigenvalues -- 0.874040 .875280 .880490 .880570 .88391 Alpha virt. eigenvalues -- 0.890960 .893960 .899220 .901610 .90522 Alpha virt. eigenvalues -- 0.907070 .914920 .920060 .922360 .94223 Alpha virt. eigenvalues -- 0.942990 .968660 .976570 .985410 .98580 Alpha virt. eigenvalues -- 0.988570 .997901 .012121 .013861 .01945 Alpha virt. eigenvalues -- 1.019701 .028181 .030011 .032851 .03995 Alpha virt. eigenvalues -- 1.043761 .044931 .056921 .057781 .06686 Alpha virt. eigenvalues -- 1.070891 .073971 .080241 .083071 .09006 Alpha virt. eigenvalues -- 1.092791 .095591 .101621 .102691 .10480 Alpha virt. eigenvalues -- 1.108881 .120571 .120611 .127291 .12838 Alpha virt. eigenvalues -- 1.130461 .130881 .134381 .134901 .13518 Alpha virt. eigenvalues -- 1.140631 .147191 .152771 .154961 .16062 Alpha virt. eigenvalues -- 1.165721 .174051 .174651 .189841 .19408 Alpha virt. eigenvalues -- 1.202691 .213791 .219661 .233101 .23349 Alpha virt. eigenvalues -- 1.245541 .248881 .261401 .276201 .27904 Alpha virt. eigenvalues -- 1.282651 .282721 .295001 .314341 .31501 Alpha virt. eigenvalues -- 1.318281 .324071 .342801 .346161 .35231 Alpha virt. eigenvalues -- 1.364761 .368711 .381021 .392711 .40318 Alpha virt. eigenvalues -- 1.413641 .416991 .430541 .441421 .46456 Alpha virt. eigenvalues -- 1.470751 .490191 .560891 .583101 .58981 
Alpha virt. eigenvalues -- 1.591431 .591481 .596741 .717321 .76630

Alpha virt. eigenvalues -- 1.774021 .83421

\section{Comparison of Experimental and DFT UV-Vis}

\section{Gas Phase}

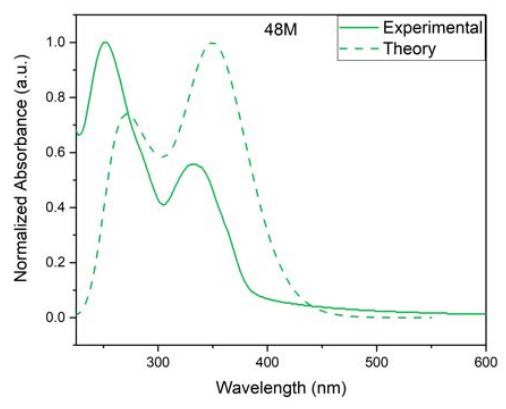

\section{Chloroform}

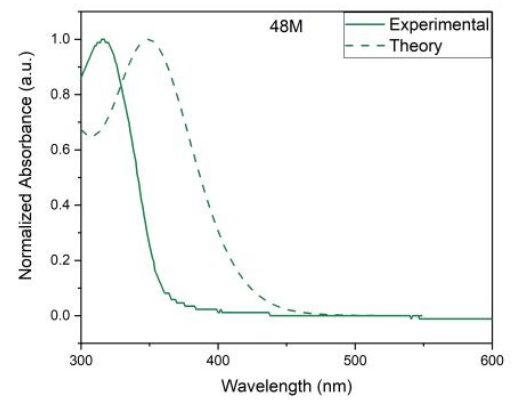

Figure S26. Gas phase and chloroform overlays of computation and experimental data for 48M

\section{Lowest 15 Excited States}

\begin{tabular}{|c|c|c|c|c|c|c|}
\hline \multicolumn{4}{|c|}{ Gas Phase } & \multicolumn{3}{|c|}{ Chloroform } \\
\hline$\frac{\text { Excited }}{\text { state }}$ & $\frac{\text { Energy }}{(\mathrm{eV})}$ & $\frac{\text { Wavelength }}{(\mathrm{nm})}$ & $f$ & $\frac{\text { Energy }}{(\mathrm{eV})}$ & $\frac{\text { Wavelength }}{(\mathrm{nm})}$ & $f$ \\
\hline 1 & 3.52 & 352.18 & 0.79 & 3.53 & 351.56 & 1.19 \\
\hline 2 & 3.53 & 350.80 & 0.27 & 3.58 & 346.12 & 0.03 \\
\hline 3 & 3.54 & 350.42 & 0.01 & 3.59 & 345.30 & 0.01 \\
\hline 4 & 3.94 & 314.80 & 0.00 & 3.97 & 312.66 & 0.00 \\
\hline 5 & 4.16 & 298.25 & 0.32 & 4.14 & 299.20 & 0.31 \\
\hline 6 & 4.20 & 295.32 & 0.07 & 4.20 & 294.85 & 0.13 \\
\hline 7 & 4.32 & 286.83 & 0.04 & 4.29 & 289.02 & 0.90 \\
\hline 8 & 4.33 & 286.52 & 0.02 & 4.30 & 288.39 & 0.05 \\
\hline 9 & 4.53 & 273.71 & 0.00 & 4.56 & 271.61 & 0.05 \\
\hline 10 & 4.53 & 273.60 & 0.00 & 4.56 & 271.60 & 0.00 \\
\hline 11 & 4.58 & 270.62 & 0.00 & 4.59 & 270.05 & 0.08 \\
\hline 12 & 4.58 & 270.53 & 0.01 & 4.59 & 269.98 & 0.00 \\
\hline 13 & 4.67 & 265.39 & 0.06 & 4.62 & 268.57 & 0.52 \\
\hline 14 & 4.67 & 265.39 & 0.06 & 4.63 & 267.87 & 0.03 \\
\hline 15 & 4.68 & 264.98 & 0.61 & 4.66 & 266.18 & 0.09 \\
\hline
\end{tabular}

Table S5. Gas phase and chloroform excitation energies, wavelengths, and oscillator strengths for first 15 excited state of 48M.

\section{Geometric Images}

Gas Phase 


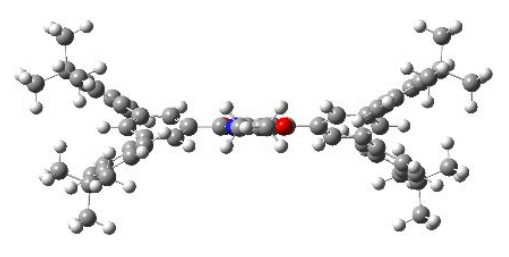

Chloroform

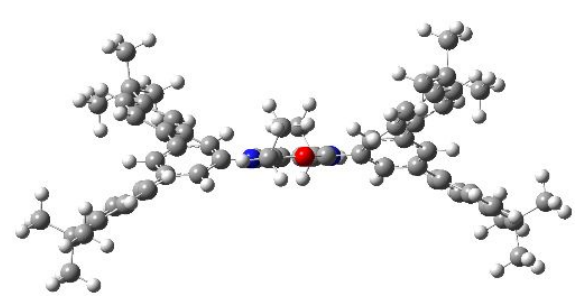

Figure S27. Geometric optimization images in gas phase and chloroform for 48M.
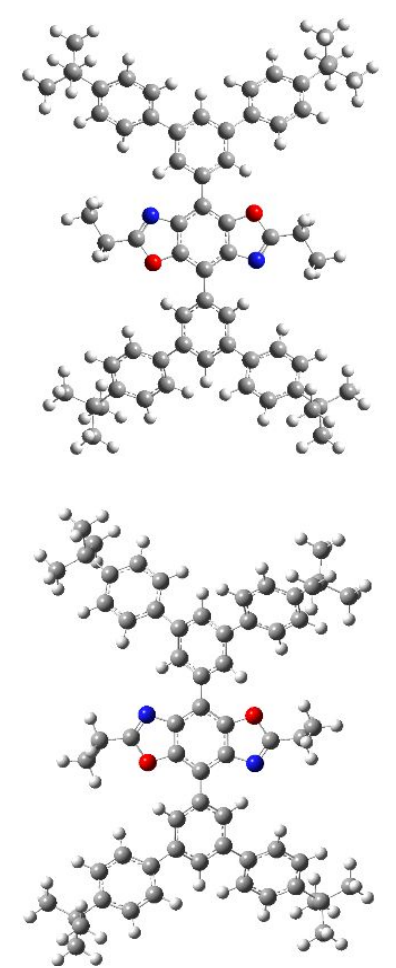

\section{P}

\section{Cartesian Coordinates}

Gas Phase

C - 1.522130 .029460 .00431

C -0.677211 .15350 .00418$

C $1.52213-0.029470 .0043$

C $0.67721-1.153520 .00423$

C 0.743931 .157790 .00823

C 0.107133 .243240 .01444

O - 1.076172 .49580 .00778

N 1.17532 .499520 .01427

C $-0.74393-1.157810 .00828$

C $-0.10713-3.243260 .01458$

$\mathrm{N}-1.1753-2.499530 .01438$

O $1.07617-2.495820 .00789$

C $3.00028-0.078940 .00016$

C $3.70382-1.2984-0.12475$

C 3.778421 .098460 .11941

C $5.10372-1.33714-0.13104$

H $3.15547-2.235-0.22003$

C 5.173381 .04650 .11494

H 3.272972 .060960 .20863

C $5.8785-0.16919-0.01097$

H $5.58671-2.31143-0.23431$

H 5.723071 .988410 .21167

C -3.00028 0.078930 .00017

C -3.70381 $1.2984-0.12473$ 
C $-3.77843-1.098460 .11941$

C -5.10372 $1.33714-0.13102$

H -3.15546 2.23499 -0.21999

C $-5.17338-1.04650 .11494$

H -3.27299 -2.06097 0.20863

C $-5.87850 .1692-0.01095$

H -5.5867 2.31144 - 0.23427

H $-5.72308-1.98840 .21166$

C $7.41643-0.17428-0.01648$

C $7.927150 .67632-1.20693$

C 7.937680 .433461 .3103

C $7.99241-1.59754-0.15593$

H $7.572810 .25892-2.16815$

H $7.576021 .72246-1.14176$

H $9.034370 .68995-1.22211$

H $7.58701-0.157032 .17753$

H 9.045 0.439551 .32003

H 7.591851 .474361 .44701

H $9.09788-1.55431-0.15458$

H $7.68167-2.247970 .68283$

H $7.67727-2.0746-1.10257$

C - $7.416430 .1743-0.01646$

C $-7.92715-0.67632-1.20689$

C -7.93768 -0.43341 1.31034

C -7.9924 1.59756 -0.15594

H $-7.57282-0.25894-2.16812$

H - $7.57603-1.72247-1.1417$

H -9.03437 -0.68995 - 1.22207

H -7.587 0.1571 2.17755

H -9.04501 -0.43948 1.32006

$\mathrm{H}-7.59187-1.474311 .44706$

H - $9.097871 .55434-0.15456$

$\mathrm{H}-7.681632 .248020 .68279$

H -7.67728 2.07459-1.1026

C -0.022524 .722370 .01837$

C 1.325695 .443620 .03355

H -0.635325 .015150 .89452$

H -0.61736 5.02119-0.86825

H 1.175556 .537710 .0317

H 1.911385 .173790 .93009

H $1.931625 .17283-0.84907$

C $0.02252-4.722390 .01858$

C $-1.32569-5.443630 .03376$

H $0.6353-5.015120 .89476$

H $0.61738-5.02124-0.86801$

H - $1.17555-6.537720 .03197$

H - $1.9114-5.173760 .93027$

H - 1.9316 -5.17289 -0.84888

Chloroform

C -0.73589 1.11919-0.11832

C $0.73589-1.11909-0.11829$

C $-0.68088-1.19841-0.10934$

$\mathrm{N}-1.04482-2.55917-0.09715$

C $0.06459-3.24735-0.09786$ 
O $1.20871-2.43473-0.10962$

C - $0.064543 .24745-0.09796$ C $0.680891 .1985-0.10936$

O - $1.208682 .43485-0.10967$

N $1.044852 .55925-0.09723$

C $0.26825-4.71639-0.08471$

H $0.77422-5.01206-1.02491$

H - $0.73215-5.17938-0.09632$

C $1.08969-5.215421 .11673$

H $1.20731-6.312281 .06416$

H $2.09717-4.762571 .12695$

H $0.59094-4.969132 .07157$

C $-0.268174 .7165-0.08488$

H - $0.774125 .01214-1.02509$

H $0.732245 .17947-0.09648$

C - 1.089625 .215591 .11653

H - 1.207216 .312451 .06392

H - 2.097124 .762781 .12675

H -0.5909 4.96932 2.07139

C $1.511210 .05074-0.11421$

C - $1.51122-0.05064-0.11423$

C $-2.988-0.07629-0.10921$

C $-3.70321-1.191920 .38633$

C $-3.744121 .0127-0.59485$

C -5.10025 - 1.204190 .39812

H -3.15303 -2.05944 0.754

C $-5.145230 .98803-0.58412$

H $-3.235631 .89193-0.99391$

C $-5.86143-0.11703-0.08524$

H -5.6051 -2.09001 0.79674

H -5.67597 $1.85727-0.97874$

C $-7.39822-0.17501-0.0516$

C $-7.87205-0.336441 .41509$

C $-7.88449-1.38753-0.88518$

C $-8.040191 .10103-0.63103$

H -8.97739 -0.3846 1.45624

H -7.4755 - 1.261021 .87316

H -7.54094 0.520812 .0313

H -8.99005 - $1.44293-0.86843$

H - $7.55958-1.29575-1.93883$

H -7.49181 -2.3415 -0.48788

H -7.75187 $2.00124-0.05685$

H -7.75826 $1.257-1.68905$

H -9.14204 $1.01456-0.58738$

C $2.987980 .07636-0.10918$

C 3.703221 .191940 .38642

C $3.74405-1.01267-0.59481$

C 5.100271 .204140 .39824

H 3.153082 .059470 .7541

C $5.14516-0.98808-0.58404$

H $3.2355-1.89187-0.99389$

C $5.861390 .11693-0.08511$

H 5.605162 .089920 .79688

H $5.67587-1.85734-0.97865$

C $7.398190 .17486-0.05146$

C 7.872020 .336491 .41521 
C $7.884491 .38722-0.88524$

C $8.04013-1.10131-0.63067$

H 8.977370 .384591 .45633

H 7.475551 .261181 .87314

H 7.54086 - 0.520632 .03156

H $8.990051 .44262-0.86848$

H $7.55961 .29525-1.93887$

H $7.491792 .34126-0.48813$

H $7.7518-2.00141-0.05632$

H $7.75821-1.25746-1.68866$

H $9.14199-1.01485-0.58703$

\section{Energy Levels}

\section{Gas Phase}

Alpha occ. eigenvalues -- -19.20861 -19.20861 -14.33308 -14.33307-10.28504

Alpha occ. eigenvalues -- -10.28503 -10.25167 -10.25155 -10.22662 -10.22651

Alpha occ. eigenvalues -- -10.21276 -10.21265 -10.19996 -10.19996-10.19252

Alpha occ. eigenvalues -- -10.19252 -10.18805 -10.18792-10.17952 -10.17951

Alpha occ. eigenvalues -- -10.17079 -10.17079 -10.16673 -10.16672 -10.16607

Alpha occ. eigenvalues -- $-10.16606-10.16558-10.16558-10.16233-10.16233$

Alpha occ. eigenvalues -- -10.15631 -10.15631 -10.15628 -10.15628 -10.15625

Alpha occ. eigenvalues -- -10.15625 -1.14091 -1.13988 -0.94807 -0.94783

Alpha occ. eigenvalues -- $-0.88606-0.86184-0.85621-0.82347-0.81952$

Alpha occ. eigenvalues -- $-0.81502-0.79813-0.78691-0.74600-0.74324$

Alpha occ. eigenvalues -- $-0.73887-0.73687-0.71540-0.69269-0.68198$

Alpha occ. eigenvalues -- $-0.68179-0.67987-0.67986-0.65359-0.65078$

Alpha occ. eigenvalues -- -0.64162 -0.61339-0.60872 -0.60750 -0.59230

Alpha occ. eigenvalues -- $-0.58511-0.58134-0.56248-0.54802-0.54669$

Alpha occ. eigenvalues -- $-0.52890-0.51675-0.51087-0.50814-0.50023$

Alpha occ. eigenvalues -- $-0.48971-0.47696-0.46721-0.46639-0.46582$

Alpha occ. eigenvalues -- $-0.46026-0.44970-0.44544-0.44531-0.44489$

Alpha occ. eigenvalues -- -0.44219-0.44217 -0.43477-0.42551 -0.42238

Alpha occ. eigenvalues -- $-0.42056-0.41703-0.40786-0.40280-0.39903$

Alpha occ. eigenvalues -- $-0.39729-0.39700-0.39658-0.38851-0.38561$

Alpha occ. eigenvalues -- $-0.38232-0.37984-0.37849-0.37724-0.37649$

Alpha occ. eigenvalues -- $-0.36763-0.36591-0.36575-0.36521-0.36359$

Alpha occ. eigenvalues -- -0.35197 -0.34975 -0.34783 -0.34659-0.33454

Alpha occ. eigenvalues -- $-0.33280-0.32879-0.32802-0.32339-0.31635$

Alpha occ. eigenvalues -- $-0.31626-0.28972-0.28466-0.28429-0.25272$

Alpha occ. eigenvalues -- $-0.25216-0.24944-0.24863-0.20876$

Alpha virt. eigenvalues -- $-0.07328-0.02514-0.01115-0.002640 .00038$

Alpha virt. eigenvalues -- 0.009660 .027040 .054620 .056790 .05838

Alpha virt. eigenvalues -- 0.059470 .060320 .088500 .089730 .09249

Alpha virt. eigenvalues -- 0.096220 .103940 .104670 .106700 .10672

Alpha virt. eigenvalues -- 0.112570 .112970 .113630 .113960 .11871

Alpha virt. eigenvalues -- 0.119540 .125110 .128950 .130070 .13257

Alpha virt. eigenvalues -- 0.137230 .138200 .141590 .155210 .15745

Alpha virt. eigenvalues -- 0.159410 .163330 .163910 .170540 .17469

Alpha virt. eigenvalues -- 0.177640 .179450 .181420 .188560 .19044

Alpha virt. eigenvalues -- 0.190590 .193610 .194530 .197560 .19926

Alpha virt. eigenvalues -- 0.203940 .205060 .210750 .211120 .21123

Alpha virt. eigenvalues -- 0.216550 .233020 .233840 .236920 .23741

Alpha virt. eigenvalues -- 0.249860 .251720 .252290 .253050 .25447

Alpha virt. eigenvalues -- 0.260130 .274160 .282410 .285910 .29435 
Alpha virt. eigenvalues -- 0.301740 .307630 .309510 .322330 .32557 Alpha virt. eigenvalues -- 0.338150 .340590 .351940 .353470 .36248 Alpha virt. eigenvalues -- 0.366830 .388390 .390210 .395250 .39606 Alpha virt. eigenvalues -- 0.417930 .423430 .423430 .429280 .43543 Alpha virt. eigenvalues -- 0.436680 .438880 .439490 .441970 .44462 Alpha virt. eigenvalues -- 0.446790 .452140 .458590 .462710 .46795 Alpha virt. eigenvalues -- 0.473400 .478490 .479200 .480450 .48244 Alpha virt. eigenvalues -- 0.486050 .491190 .492750 .498630 .50263 Alpha virt. eigenvalues -- 0.507650 .508970 .510070 .521430 .53144 Alpha virt. eigenvalues -- 0.536100 .538040 .548460 .553880 .55771 Alpha virt. eigenvalues -- 0.566970 .569350 .571460 .574160 .57879 Alpha virt. eigenvalues -- 0.581510 .586400 .592660 .600920 .60555 Alpha virt. eigenvalues -- 0.611810 .614920 .625210 .628820 .63332 Alpha virt. eigenvalues -- 0.637060 .640370 .648620 .650660 .65499 Alpha virt. eigenvalues -- 0.655150 .657760 .666600 .670180 .67135 Alpha virt. eigenvalues -- 0.675890 .677300 .678370 .679650 .68014 Alpha virt. eigenvalues -- 0.682650 .684110 .686500 .687410 .68846 Alpha virt. eigenvalues -- 0.691000 .693910 .694890 .695400 .69862 Alpha virt. eigenvalues -- 0.702620 .703350 .703700 .708570 .71128 Alpha virt. eigenvalues -- 0.712070 .713310 .717860 .719400 .72141 Alpha virt. eigenvalues -- 0.724410 .725030 .726380 .726920 .72733 Alpha virt. eigenvalues -- 0.734170 .742230 .742910 .745210 .74731 Alpha virt. eigenvalues -- 0.748370 .755980 .759950 .762060 .76225 Alpha virt. eigenvalues -- 0.766350 .767260 .769950 .775270 .77726 Alpha virt. eigenvalues -- 0.783600 .787130 .798310 .803010 .80539 Alpha virt. eigenvalues -- 0.807020 .809960 .812080 .815220 .81731 Alpha virt. eigenvalues -- 0.820270 .823850 .841420 .845260 .84636 Alpha virt. eigenvalues -- 0.855870 .857970 .859790 .863310 .87321 Alpha virt. eigenvalues -- 0.879080 .900390 .917990 .924600 .94598 Alpha virt. eigenvalues -- 0.952460 .963560 .972450 .981931 .00883 Alpha virt. eigenvalues -- 1.008971 .017991 .027411 .033091 .05794 Alpha virt. eigenvalues -- 1.058391 .065641 .077831 .085441 .08976 Alpha virt. eigenvalues -- 1.101691 .118021 .118021 .129561 .13065 Alpha virt. eigenvalues -- 1.140771 .144281 .173991 .176881 .19192 Alpha virt. eigenvalues -- 1.199961 .232211 .259401 .275371 .27897 Alpha virt. eigenvalues -- 1.288871 .293591 .310621 .326401 .33786 Alpha virt. eigenvalues -- 1.362281 .363531 .375081 .397551 .41750 Alpha virt. eigenvalues -- 1.438281 .448541 .464881 .495371 .52235 Alpha virt. eigenvalues -- 1.534851 .582391 .631071 .747151 .77322 Alpha virt. eigenvalues -- 1.777521 .86691

\section{Chloroform}

Alpha occ. eigenvalues -- -19.21316 -19.21315 -14.33810 -14.33809 -10.28879

Alpha occ. eigenvalues -- -10.28878 -10.25716 -10.25705 -10.23204 -10.23195

Alpha occ. eigenvalues -- -10.21883 -10.21873 -10.19769 -10.19756 -10.19569 Alpha occ. eigenvalues -- -10.19569 -10.19493 -10.19493 -10.18817 - 10.18817 Alpha occ. eigenvalues -- -10.17813 -10.17813 -10.17591 -10.17591 -10.17510 Alpha occ. eigenvalues -- -10.17510 - 10.17509 -10.17509 -10.17100 -10.17100 Alpha occ. eigenvalues -- -10.15816 -10.15816 -10.15805 -10.15805 -10.15694 Alpha occ. eigenvalues -- -10.15694 -1.14476 -1.14369 -0.95198 -0.95187 Alpha occ. eigenvalues -- -0.89318 -0.87120 -0.86432 -0.82930 -0.82000 Alpha occ. eigenvalues -- $-0.81991-0.79797-0.79284-0.75092-0.75062$ Alpha occ. eigenvalues -- -0.74893 -0.74348 -0.72302 -0.68820 -0.68405 Alpha occ. eigenvalues -- $-0.68372-0.68192-0.68191-0.65970-0.65079$ Alpha occ. eigenvalues -- -0.65072 -0.62110 -0.61518 -0.61121 -0.59945 
Alpha occ. eigenvalues -- -0.59531 -0.59082 -0.56501 -0.55339 -0.55311 Alpha occ. eigenvalues -- $-0.53162-0.52236-0.51639-0.50797-0.49408$ Alpha occ. eigenvalues -- -0.48943 -0.48202 -0.47185 -0.46980 -0.46972 Alpha occ. eigenvalues -- $-0.46442-0.45283-0.45225-0.44801-0.44528$ Alpha occ. eigenvalues -- -0.44424 -0.44422 -0.44319-0.43000 -0.42933 Alpha occ. eigenvalues -- -0.42817 -0.42367 -0.41648 -0.41278 -0.40887 Alpha occ. eigenvalues -- -0.40136 -0.39890 -0.39876 -0.39450 -0.38482 Alpha occ. eigenvalues -- $-0.38338-0.38119-0.37843-0.37744-0.37556$ Alpha occ. eigenvalues -- $-0.37296-0.36730-0.36703-0.36351-0.36294$ Alpha occ. eigenvalues -- $-0.35767-0.35678-0.35431-0.35426-0.33748$ Alpha occ. eigenvalues -- $-0.33606-0.33206-0.33179-0.32681-0.32169$ Alpha occ. eigenvalues -- -0.32119-0.29984 -0.29479 -0.28837 -0.26310 Alpha occ. eigenvalues -- $-0.26263-0.25769-0.25475-0.21924$ Alpha virt. eigenvalues -- $-0.07785-0.03182-0.01828-0.01267-0.01127$ Alpha virt. eigenvalues -- -0.001120 .016830 .045200 .054800 .05868$ Alpha virt. eigenvalues -- 0.061730 .063830 .091630 .093590 .09699 Alpha virt. eigenvalues -- 0.097980 .104040 .104700 .105700 .10903 Alpha virt. eigenvalues -- 0.110280 .111740 .114310 .115540 .11807 Alpha virt. eigenvalues -- 0.119480 .121570 .128190 .130390 .13278 Alpha virt. eigenvalues -- 0.133790 .138370 .141110 .143240 .14880 Alpha virt. eigenvalues -- 0.150260 .153290 .155060 .166590 .17181 Alpha virt. eigenvalues -- 0.173900 .174360 .176110 .177560 .18568 Alpha virt. eigenvalues -- 0.186850 .187400 .191610 .191960 .19477 Alpha virt. eigenvalues -- 0.195690 .202860 .203280 .205940 .20769 Alpha virt. eigenvalues -- 0.219730 .225590 .231080 .234370 .23890 Alpha virt. eigenvalues -- 0.243910 .247110 .249250 .249450 .25687 Alpha virt. eigenvalues -- 0.263170 .266750 .276130 .283810 .28501 Alpha virt. eigenvalues -- 0.296920 .297160 .303820 .312860 .31779 Alpha virt. eigenvalues -- 0.326040 .329380 .335410 .340740 .35140 Alpha virt. eigenvalues -- 0.353200 .378440 .383360 .386460 .38885 Alpha virt. eigenvalues -- 0.411700 .415960 .421590 .421610 .42605 Alpha virt. eigenvalues -- 0.430470 .433960 .436110 .438970 .44139 Alpha virt. eigenvalues -- 0.447250 .449420 .452400 .456730 .46327 Alpha virt. eigenvalues -- 0.464610 .468100 .470020 .471070 .47934 Alpha virt. eigenvalues -- 0.484950 .486020 .488100 .488920 .49307 Alpha virt. eigenvalues -- 0.498710 .499220 .501830 .511610 .51413 Alpha virt. eigenvalues -- 0.520210 .524580 .541030 .550760 .55773 Alpha virt. eigenvalues -- 0.557930 .565290 .569600 .571160 .57511 Alpha virt. eigenvalues -- 0.578910 .582040 .582430 .595410 .59986 Alpha virt. eigenvalues -- 0.608890 .610320 .612350 .623760 .62666 Alpha virt. eigenvalues -- 0.626730 .632230 .642220 .648450 .64859 Alpha virt. eigenvalues -- 0.652010 .652670 .657700 .664290 .66693 Alpha virt. eigenvalues -- 0.671860 .673120 .674320 .679100 .67949 Alpha virt. eigenvalues -- 0.682890 .684350 .685020 .687680 .68989 Alpha virt. eigenvalues -- 0.690070 .691890 .694650 .696080 .69744 Alpha virt. eigenvalues -- 0.699560 .701580 .703120 .704790 .70755 Alpha virt. eigenvalues -- 0.710820 .714650 .714670 .719770 .72265 Alpha virt. eigenvalues -- 0.723530 .725110 .725770 .726640 .73213 Alpha virt. eigenvalues -- 0.732830 .732930 .733870 .741180 .74174 Alpha virt. eigenvalues -- 0.746030 .746600 .750360 .755800 .76002 Alpha virt. eigenvalues -- 0.761770 .762460 .763230 .767290 .78043 Alpha virt. eigenvalues -- 0.784760 .788770 .793630 .796630 .80022 Alpha virt. eigenvalues -- 0.801360 .810250 .813930 .815840 .81782 Alpha virt. eigenvalues -- 0.824030 .838510 .838570 .843890 .84614 Alpha virt. eigenvalues -- 0.846980 .847180 .859000 .859100 .87327 Alpha virt. eigenvalues -- 0.882340 .890200 .901920 .902040 .90596 
Alpha virt. eigenvalues -- 0.919150 .943630 .946800 .960960 .98095

Alpha virt. eigenvalues -- 0.983691 .005541 .019721 .024851 .03311

Alpha virt. eigenvalues -- 1.038421 .056731 .057971 .067951 .08656

Alpha virt. eigenvalues -- 1.101261 .102101 .117611 .120011 .12281

Alpha virt. eigenvalues -- 1.131751 .132501 .136121 .153891 .15405

Alpha virt. eigenvalues -- 1.163701 .189731 .211901 .216861 .23490

Alpha virt. eigenvalues -- 1.250001 .251851 .262841 .277411 .29448

Alpha virt. eigenvalues -- 1.303321 .316891 .331341 .347921 .37554

Alpha virt. eigenvalues -- 1.382711 .394231 .423341 .424291 .47420

Alpha virt. eigenvalues -- 1.502861 .579131 .599081 .712731 .76347

Alpha virt. eigenvalues -- 1.767231 .83677

\section{Comparison of Experimental and DFT UV-Vis}

Gas Phase

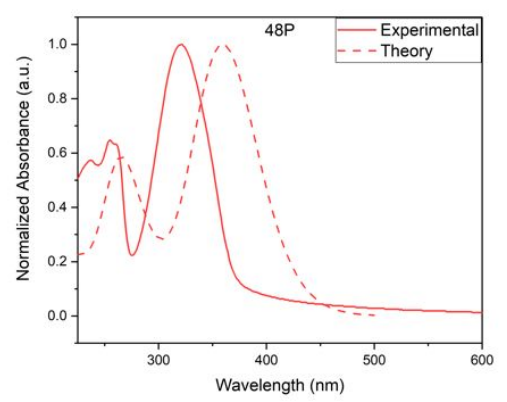

Chloroform

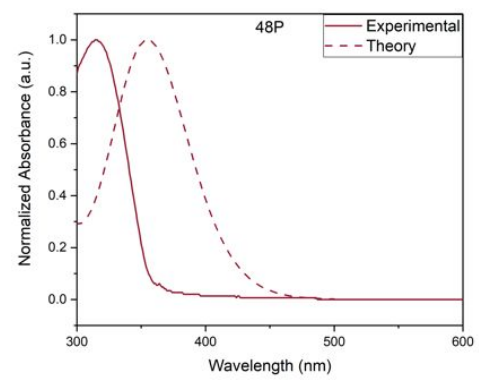

Figure S28. Gas phase and chloroform overlays of computation and experimental data for 48P.

\section{Lowest 15 Excited States}

\begin{tabular}{|c|c|c|c|c|c|c|}
\hline \multicolumn{4}{|c|}{ Gas Phase } & \multicolumn{3}{|c|}{ Chloroform } \\
\hline$\frac{\text { Excited }}{\text { state }}$ & $\begin{array}{l}\text { Energy } \\
(\mathrm{eV})\end{array}$ & $\frac{\text { Wavelength }}{(\mathrm{nm})}$ & $f$ & $\frac{\text { Energy }}{(\mathrm{eV})}$ & $\frac{\text { Wavelength }}{(\mathrm{nm})}$ & $f$ \\
\hline 1 & 3.44 & 359.90 & 1.06 & 3.49 & 355.46 & 1.24 \\
\hline 2 & 4.13 & 300.44 & 0.12 & 4.17 & 297.48 & 0.15 \\
\hline 3 & 4.27 & 290.32 & 0.00 & 4.39 & 282.71 & 0.00 \\
\hline 4 & 4.31 & 287.73 & 0.06 & 4.42 & 280.40 & 0.03 \\
\hline 5 & 4.35 & 285.85 & 0.00 & 4.44 & 279.47 & 0.00 \\
\hline 6 & 4.68 & 264.67 & 0.56 & 4.68 & 265.02 & 0.63 \\
\hline 7 & 4.85 & 255.49 & 0.00 & 4.94 & 251.08 & 0.00 \\
\hline 8 & 4.87 & 254.57 & 0.00 & 5.01 & 247.36 & 0.00 \\
\hline 9 & 5.00 & 247.86 & 0.00 & 5.07 & 244.66 & 0.04 \\
\hline 10 & 5.09 & 243.38 & 0.00 & 5.07 & 244.40 & 0.01 \\
\hline 11 & 5.13 & 241.64 & 0.06 & 5.14 & 241.20 & 0.10 \\
\hline 12 & 5.20 & 238.27 & 0.05 & 5.20 & 238.40 & 0.01 \\
\hline 13 & 5.40 & 229.64 & 0.00 & 5.38 & 230.45 & 0.00 \\
\hline 14 & 5.59 & 221.71 & 0.00 & 5.58 & 222.29 & 0.00 \\
\hline 15 & 5.62 & 220.70 & 0.19 & 5.61 & 221.01 & 0.00 \\
\hline
\end{tabular}

Table S6. Gas phase and chloroform excitation energies, wavelengths, and oscillator strengths for first 15 excited state of $48 \mathrm{P}$. 


\section{Geometric Images}
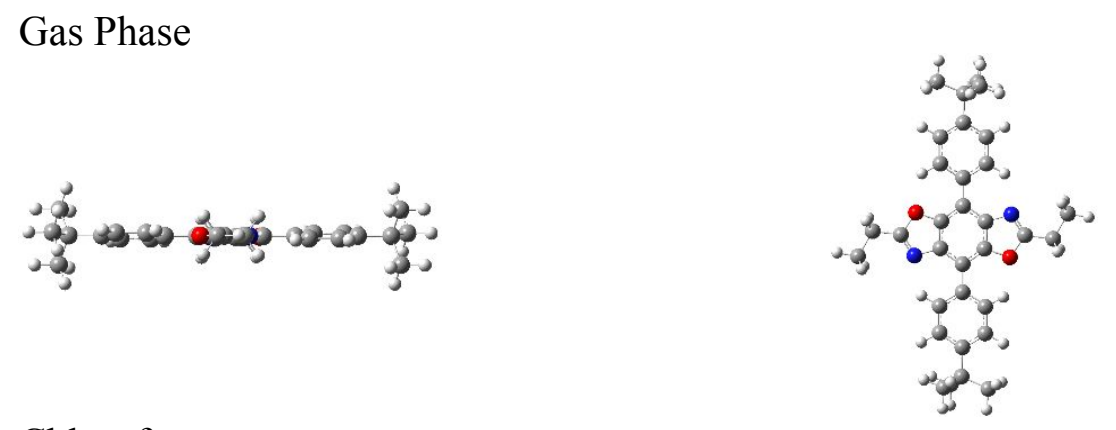

\section{Chloroform}
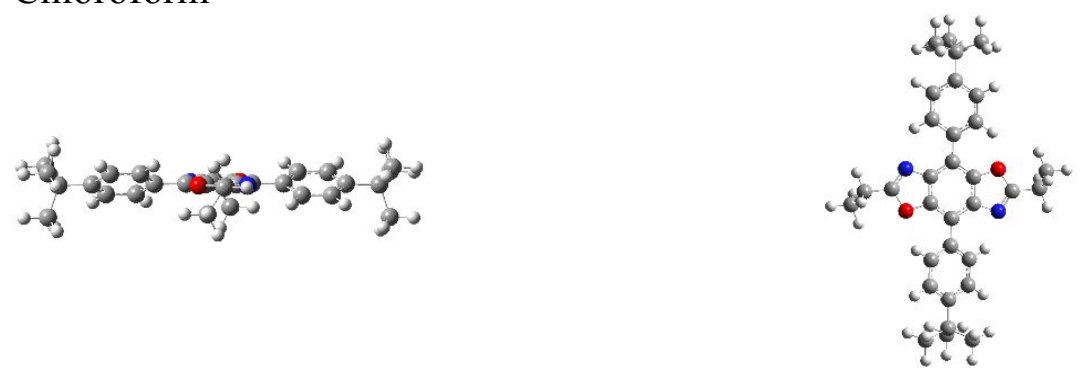

Figure S29. Geometric optimization images in gas phase and chloroform for 48P.

\section{M48P}

\section{Cartesian Coordinates}

\section{Gas Phase}

C -0.077481 .509270 .01184$

C -1.178810 .641890 .01176$

C $0.0688-1.51423-0.00679$

C $1.17017-0.64686-0.00213$

C $-1.14386-0.77984-0.0074$

C $-3.24153-0.19914-0.01097$

O -2.526881 .007140 .00928$

$\mathrm{N}-2.46284-1.25245-0.01944$

C $1.135150 .77502-0.0047$

C $3.232760 .19451-0.026$

N $2.454031 .24781-0.01867$

O $2.51821-1.01194-0.01568$

C $0.16185-2.98717-0.01139$

C $1.29758-3.651640 .49795$

C $-0.88269-3.78858-0.52691$

C $1.38631-5.049360 .49042$

H 2.12186 -3.0721 0.91741

C $-0.78051-5.18132-0.53686$

H - $1.78391-3.30951-0.91266$

C $0.3538-5.85157-0.03$

H $2.28631-5.507890 .90578$

H - $1.61343-5.75707-0.9532$

C -0.170442 .982120 .02885$

C -1.302563 .638940 .55586$

C $0.871193 .79108-0.48067$ 
C -1.390125 .036650 .57203$

H -2.12462 3.053180 .97104

C $0.770095 .18388-0.46696$

H $1.769573 .31773-0.87988$

C -0.360065 .846560 .0587$

H - 2.287 5.488911 .00076

H $1.600725 .7659-0.87905$

C $0.41925-7.38825-0.06174$

C $-0.76191-7.969680 .7554$

C $0.31899-7.87467-1.52953$

C $1.73376-7.926840 .5377$

H - $0.7133-7.634251 .80841$

H - $1.738-7.654110 .34367$

H - $0.72982-9.076550 .74227$

H $1.15462-7.47373-2.13342$

H $0.35979-8.98053-1.57192$

H - $0.62637-7.55166-2.00233$

H $1.73712-9.032280 .49415$

H 2.61665 -7.56733 -0.02319

H $1.85102-7.633151 .59744$

C - 0.423287 .383640 .05498

C 0.762847 .947940 .87698

C $-0.329257 .89695-1.40404$

C -1.733967 .913190 .67075$

H 0.719597 .591721 .92339

H 1.736397 .639760 .45376

H 0.731699 .054880 .88602

H - $1.16867 .5089-2.01118$

H $-0.368119 .00346-1.42554$

H $0.613167 .58118-1.88761$

H -1.73613 9.01924 0.64681

H -2.62031 7.56471 0.10838

H - 1.846097 .600971 .72574

C $-4.69277-0.13739-0.0134$

C -5.42847-1.33678 0.01643

C $-5.359221 .10076-0.04306$

C -6.83377-1.31139 0.01997

$\mathrm{H}-4.88276-2.283740 .01642$

C -6.76536 $1.15351-0.04084$

H $-4.772352 .02233-0.0461$

C $-7.61777-2.573370 .04838$

C $-7.48238-0.05903-0.00865$

C -7.47551 $2.45866-0.06256$

C -7.16652 -3.69788 0.77155

C $-8.83846-2.6949-0.64191$

H $-8.57566-0.02791-0.0052$

C -8.677572 .652280 .64383$

C $-6.968143 .55444-0.79268$

C $-7.90836-4.882010 .80078$

H -6.23326 -3.63779 1.34018

C $-9.5791-3.88476-0.6094$

H $-9.20934-1.85622-1.23957$

C -9.345333 .884750 .62201$

H -9.089611 .836521 .24628$

C $-7.637664 .78115-0.81165$

H -6.04877 3.43874 -1.37527 
C -9.13497 -5.00767 0.11362

H -7.52147 -5.72406 1.38333

H - $10.5154-3.92493-1.17024$

C -8.84417 $4.98003-0.10628$

H - 10.269363 .98091 .19624

H -7.20929 5.59849 -1.40052

C $4.683970 .13288-0.03446$

C $5.41971 .3324-0.00873$

C $5.35055-1.10522-0.06262$

C $6.824991 .30726-0.00795$

H $4.87392 .2793-0.00931$

C $6.75667-1.1577-0.06099$

H $4.76435-2.02721-0.06188$

C 7.608852 .569440 .01491

C $7.473760 .05486-0.03312$

C $7.46645-2.46313-0.07427$

C 7.15893 .696310 .73521

C $8.828052 .68882-0.67838$

H $8.567080 .02387-0.02959$

C $8.66511-2.653330 .64531$

C $6.96641-3.56003-0.80123$

C 7.900544 .880690 .75874

H 6.226893 .637971 .30605

C $9.568483 .87896-0.6516$

H $9.197811 .84815-1.27394$

C $9.32518-3.885330 .63564$

H $9.07273-1.833881 .24575$

C $7.63273-4.79344-0.80883$

H $6.0526-3.44652-1.39305$

C 9.125625 .004270 .06847

H 7.51472 5.724651 .33922

H $10.503573 .91743-1.2146$

C $8.82758-4.98867-0.0908$

H $10.24567-3.986861 .21932$

H 7.20402 -5.60759 -1.39725

C $-9.92051-6.329570 .17774$

C $-9.05137-7.472-0.40554$

C $-10.26872-6.648911 .65345$

C $-11.23535-6.26314-0.62458$

H -8.7914 -7.26814 -1.46127

H -8.10837 -7.59667 0.1578

H $-9.6011-8.43226-0.36351$

H - 10.89003 -5.84609 2.09294

H - $10.83453-7.598581 .71662$

H $-9.36125-6.754822 .27587$

H - $11.76827-7.22926-0.54577$

H -11.91046 -5.47651 -0.23904

H - $11.05121-6.07026-1.69793$

C $-9.549146 .34708-0.1573$

C -8.599137 .43620 .40128$

C $-9.910556 .68326-1.62617$

C -10.846526 .362820 .67528$

$\mathrm{H}-8.326167 .218871 .45101$

H -7.66471 7.50351 -0.18551

H -9.091088 .427660 .36954$

H -10.58759 5.91768 -2.04943 
H -10.42071 $7.66451-1.68029$

H -9.01198 6.73399 -2.26816

H - 11.319117 .36080 .60926

H - 11.579225 .621390 .30562

H - 10.649976 .15661 .74395

C $9.5846-6.32837-0.0684$

C $9.66198-6.848361 .38924$

C $8.88992-7.40232-0.92913$

C $11.0184-6.11909-0.61728$

H $10.192-6.137712 .04963$

H 8.6488 -7.00626 1.80413

H $10.20509-7.812671 .42395$

H $8.82611-7.09812-1.99055$

H $9.46745-8.34481-0.88585$

H $7.86811-7.62022-0.56636$

H $11.57601-7.07555-0.60627$

H $10.99015-5.74703-1.65865$

H $11.58662-5.3898-0.01138$

C 9.910916 .32660 .12635

C $9.04017 .46682-0.45873$

C 10.262356 .650921 .60022

C $11.223936 .25789-0.67875$

H 8.7779 7.25944 -1.51322

H 8.098297 .5930 .10626

H 9.58959 8.4274 -0.42103

H 10.885065 .849832 .0409

H 10.827817 .601081 .65905

H 9.356256 .758412 .22436

H $11.756787 .2244-0.60429$

H $11.900145 .4727-0.2922$

H $11.037386 .06149-1.75103$

\section{Chloroform}

C $1.17615-0.64185-0.05028$

C $-1.176150 .64185-0.05028$

C - $1.13901-0.77915-0.04337$

N -2.45748 - $1.254-0.03409$

O - $2.523921 .00595-0.0381$

C $1.139010 .77915-0.04338$

O $2.52392-1.00595-0.03811$

N $2.457481 .254-0.0341$

C $-0.075581 .50915-0.04758$

C $0.07558-1.50915-0.04758$

C $0.1726-2.98204-0.04726$

C $-0.83804-3.785470 .52952$

C $1.2799-3.6427-0.62069$

C $-0.73346-5.178840 .5322$

H - $1.71395-3.309650 .97395$

C $1.37275-5.04098-0.61781$

H $2.07778-3.0611-1.08702$

C $0.37229-5.84598-0.03998$

H - $1.53921-5.757140 .9953$

H $2.24907-5.49756-1.08278$

C $0.44105-7.38244-0.01619$

C $-0.77421-7.9603-0.78504$ 
C $0.40254-7.875511 .45253$

C $1.72967-7.9165-0.67301$

H - $0.7417-9.06694-0.77386$

H - $1.73213-7.64464-0.33243$

H $-0.76712-7.62538-1.83956$

H $0.44853-8.981191 .48463$

H $1.26275-7.477522 .02333$

H - $0.52404-7.560361 .96654$

H $1.79956-7.62236-1.7368$

H $2.6353-7.55527-0.15079$

H $1.73668-9.02177-0.6301$

C $-0.17262 .98203-0.04726$

C 0.838043 .785470 .52951

C $-1.27993 .6427-0.62069$

C 0.733465 .178840 .53219

H 1.713963 .309650 .97395

C - $1.372755 .04098-0.61781$

H -2.07778 3.06109 -1.08702

C $-0.372295 .84598-0.03998$

H 1.539215 .757140 .99529

H - $2.249075 .49756-1.08278$

C $-0.441057 .38244-0.0162$

C $0.774217 .9603-0.78504$

C -0.402547 .875511 .45252$

C $-1.729677 .9165-0.67301$

H $0.74179 .06693-0.77387$

H $1.732127 .64464-0.33244$

H $0.767127 .62538-1.83957$

H - 0.448538 .981191 .48462

H - 1.262757 .477522 .02333

H 0.524047 .560371 .96653

H - $1.799577 .62236-1.7368$

H -2.6353 7.55527 -0.15079

H - $1.736689 .02177-0.63011$

C $3.238010 .20054-0.02857$

C -3.23801-0.20054-0.02857

C - $4.68939-0.13924-0.01613$

C -5.354881 .098650 .04849$

C $-5.42607-1.33782-0.06605$

C -6.76141 .151110 .06581$

H -4.768322 .018120 .11265$

C $-6.83183-1.31251-0.05152$

$\mathrm{H}-4.88421-2.28384-0.1416$

C -7.47982 -0.060770 .01571$

C -7.470832 .454750 .14674$

C $-7.615-2.57407-0.11834$

H $-8.57297-0.029230 .02885$

C -6.96715 $3.60242-0.49578$

C -8.673762 .590980 .87279$

C - $7.15482-3.753910 .49802$

C $-8.84578-2.63781-0.80676$

C -7.6356 4.833 -0.41802

H -6.04939 3.5352 - 1.08852

C -9.335023 .820760 .94976$

H -9.087141 .730441 .40812$

C $-7.89003-4.946250 .42902$ 
$\mathrm{H}-6.217-3.742631 .06224$

C $-9.57442-3.82954-0.87415$

H $-9.22923-1.75115-1.32127$

C -8.835054 .97440 .30623$

H -7.2047 $5.68778-0.94372$

$\mathrm{H}-10.258593 .878271 .53443$

C $-9.11688-5.01573-0.25912$

$\mathrm{H}-7.48873-5.828520 .93219$

H -10.51782 -3.83054 -1.4291

C -9.594016 .308230 .41988$

C -9.94716 -6.30704 -0.36406

C -11.031 $6.13264-0.13247$

C -8.90534 $7.43751-0.37217$

C -9.664136 .730241 .90959$

C $-10.09335-6.70091-1.85578$

C $-9.28896-7.48240 .38572$

C - $11.35232-6.06740 .24398$

H -11.59519 5.365 0.42824

H - $11.587517 .08634-0.05361$

H -11.00928 5.83238 -1.19713

H $-8.845577 .2032-1.45145$

H -9.48559 8.37298 -0.26411

$\mathrm{H}-7.88247 .63418-0.00058$

$\mathrm{H}-10.184725 .973472 .52484$

$\mathrm{H}-8.648936 .870862 .32637$

H - 10.214287 .685732 .0095

H -10.69332 -7.62673-1.94828

H -10.59851 -5.91014 -2.44039

H $-9.10246-6.88426-2.3125$

H $-9.17889-7.268921 .46534$

$\mathrm{H}-9.91927-8.385780 .28547$

H -8.29122 -7.72361 -0.02595

H $-11.96082-6.989280 .17083$

$\mathrm{H}-11.27665-5.787791 .31183$

H -11.89537 -5.26153 -0.28305

C $4.689390 .13924-0.01614$

C $5.35488-1.098650 .04848$

C $5.426071 .33782-0.06606$

C 6.7614 -1.151110.0658

H $4.76833-2.018120 .11264$

C $6.831831 .31251-0.05154$

H $4.884212 .28384-0.14161$

C 7.479820 .060770 .0157

C $7.47083-2.454750 .14673$

C $7.6152 .57407-0.11836$

H 8.572970 .029230 .02883

C $6.96715-3.60242-0.4958$

C $8.67376-2.590980 .87278$

C 7.154823 .753910 .49801

C $8.845772 .63781-0.80678$

C $7.6356-4.833-0.41803$

H $6.04939-3.5352-1.08853$

C $9.33502-3.820760 .94974$

H $9.08714-1.730441 .40811$

C 7.890034 .946250 .42901

H 6.2173 .742631 .06223 
C $9.574423 .82955-0.87417$

H $9.229231 .75115-1.32129$

C $8.83505-4.97440 .30621$

H $7.2047-5.68778-0.94373$

H $10.2586-3.878271 .53441$

C $9.116885 .01574-0.25914$

H 7.488735 .828520 .93218

H $10.517813 .83054-1.42912$

C $9.59401-6.308230 .41986$

C $9.947166 .30704-0.36408$

C $11.031-6.13264-0.13249$

C $8.90534-7.43751-0.37218$

C $9.66414-6.730241 .90957$

C $10.093356 .70092-1.8558$

C 9.288967 .48240 .3857

C 11.352326 .06740 .24396

H $11.59519-5.3650 .42822$

H $11.58751-7.08634-0.05363$

H $11.00928-5.83238-1.19715$

H $8.84556-7.2032-1.45147$

H $9.48559-8.37298-0.26413$

H $7.8824-7.63418-0.0006$

H $10.18472-5.973472 .52482$

H $8.64893-6.870862 .32635$

H $10.21428-7.685732 .00948$

H $10.693317 .62673-1.9483$

H $10.59855 .91014-2.44041$

H $9.102456 .88426-2.31251$

H 9.178897 .268921 .46532

H 9.919278 .385780 .28546

H $8.291227 .72361-0.02596$

H 11.960826 .989280 .17081

H 11.276665 .787791 .31181

H $11.895375 .26153-0.28307$

\section{Energy Levels}

\section{Gas Phase}

Alpha occ. eigenvalues -- -19.20823 -19.20821 -14.33184 -14.33183 -10.28438

Alpha occ. eigenvalues -- -10.28437 -10.25138 -10.25127 -10.22626 -10.22617

Alpha occ. eigenvalues -- -10.21059 -10.21049 -10.20521 -10.20520 -10.19788

Alpha occ. eigenvalues -- -10.19787 -10.19753 -10.19752 -10.19737-10.19733

Alpha occ. eigenvalues -- -10.19721 -10.19717-10.19308-10.19305 -10.19003

Alpha occ. eigenvalues -- -10.19002 -10.18934 -10.18931 -10.18825-10.18812

Alpha occ. eigenvalues -- -10.18722 -10.18722 -10.18708 -10.18706 -10.18643

Alpha occ. eigenvalues -- -10.18640 -10.18569 -10.18567 -10.18566 -10.18561

Alpha occ. eigenvalues -- -10.18071 -10.18070 -10.17931 -10.17884-10.17876

Alpha occ. eigenvalues -- -10.17834 -10.17817-10.17813-10.17801-10.17798

Alpha occ. eigenvalues -- -10.17696 -10.17671 -10.17608 -10.17593 -10.17590

Alpha occ. eigenvalues -- -10.17589 -10.17547 -10.17543 -10.16997-10.16996

Alpha occ. eigenvalues -- -10.16762 -10.16761 -10.16748 -10.16745 -10.16688

Alpha occ. eigenvalues -- -10.16684 -10.16097 -10.16090 -10.16088 -10.16086

Alpha occ. eigenvalues -- -10.16078 -10.16067 -10.16041 -10.16037-10.16035

Alpha occ. eigenvalues -- -10.16033 -10.16032 -10.16029-10.15674-10.15668

Alpha occ. eigenvalues -- -10.15668 -10.15666 -10.15665 -10.15665 -1.14010

Alpha occ. eigenvalues -- -1.13906 -0.94738 -0.94728 -0.89298 -0.88755 
Alpha occ. eigenvalues -- -0.88084 -0.87046 -0.87043 -0.86362 -0.86302 Alpha occ. eigenvalues -- $-0.86223-0.85585-0.82720-0.82716-0.82473$ Alpha occ. eigenvalues -- $-0.82415-0.82171-0.81459-0.80297-0.78813$ Alpha occ. eigenvalues -- $-0.78752-0.78270-0.78230-0.75266-0.75005$ Alpha occ. eigenvalues -- $-0.74958-0.74930-0.74848-0.74468-0.74106$ Alpha occ. eigenvalues -- $-0.74026-0.73349-0.72635-0.72130-0.72120$ Alpha occ. eigenvalues -- $-0.71535-0.68933-0.68688-0.68664-0.68637$ Alpha occ. eigenvalues -- -0.68464 -0.68456 -0.68443 -0.68408 -0.68404 Alpha occ. eigenvalues -- $-0.68252-0.68227-0.68035-0.68032-0.65917$ Alpha occ. eigenvalues -- $-0.64785-0.64564-0.64473-0.63637-0.63222$ Alpha occ. eigenvalues -- $-0.62478-0.61440-0.60966-0.60915-0.60892$ Alpha occ. eigenvalues -- $-0.60811-0.60280-0.59641-0.59281-0.58622$ Alpha occ. eigenvalues -- $-0.58389-0.58265-0.57652-0.55894-0.55611$ Alpha occ. eigenvalues -- -0.55599 -0.55518 - $0.55512-0.55286-0.54927$ Alpha occ. eigenvalues -- $-0.54775-0.54243-0.51769-0.51323-0.51088$ Alpha occ. eigenvalues -- $-0.50369-0.50336-0.49925-0.49864-0.49807$ Alpha occ. eigenvalues -- $-0.49748-0.49063-0.48510-0.48412-0.47577$ Alpha occ. eigenvalues -- $-0.47439-0.47357-0.47245-0.47234-0.46629$ Alpha occ. eigenvalues -- -0.46314 -0.45940 -0.45799-0.45681 -0.45607 Alpha occ. eigenvalues -- -0.45579 -0.45219 -0.45159-0.44872 -0.44847 Alpha occ. eigenvalues -- $-0.44705-0.44702-0.44656-0.44649-0.44444$ Alpha occ. eigenvalues -- $-0.44292-0.44268-0.44217-0.44153-0.43887$ Alpha occ. eigenvalues -- $-0.43283-0.43217-0.43132-0.43066-0.42634$ Alpha occ. eigenvalues -- $-0.42618-0.42530-0.42501-0.42427-0.42324$ Alpha occ. eigenvalues -- $-0.42180-0.41869-0.41088-0.40809-0.40429$ Alpha occ. eigenvalues -- $-0.40260-0.40103-0.40088-0.40059-0.40015$ Alpha occ. eigenvalues -- -0.39913 -0.39906 -0.39758 -0.39662 -0.39578 Alpha occ. eigenvalues -- $-0.39438-0.38932-0.38365-0.38304-0.38289$ Alpha occ. eigenvalues -- $-0.38215-0.38033-0.37993-0.37925-0.37720$ Alpha occ. eigenvalues -- $-0.37679-0.37581-0.37215-0.37179-0.37008$ Alpha occ. eigenvalues -- $-0.37003-0.36911-0.36906-0.36781-0.36602$ Alpha occ. eigenvalues -- -0.36592 -0.36523 -0.36468 -0.36289 -0.36202 Alpha occ. eigenvalues -- -0.35536 -0.35489 -0.35397-0.35382 -0.35374 Alpha occ. eigenvalues -- $-0.35161-0.34940-0.34929-0.34133-0.33950$ Alpha occ. eigenvalues -- $-0.33944-0.33785-0.33525-0.33351-0.33222$ Alpha occ. eigenvalues -- $-0.33191-0.33144-0.33076-0.33048-0.32815$ Alpha occ. eigenvalues -- $-0.32711-0.32417-0.32412-0.32344-0.32327$ Alpha occ. eigenvalues -- -0.31824 -0.31767 -0.30175 -0.29357 -0.29193 Alpha occ. eigenvalues -- $-0.29185-0.28820-0.28041-0.27778-0.26294$ Alpha occ. eigenvalues -- $-0.26292-0.26219-0.26213-0.25364-0.25307$ Alpha occ. eigenvalues -- $-0.24899-0.24813-0.23892-0.23174-0.23169$ Alpha occ. eigenvalues -- $-0.22957-0.20950$

Alpha virt. eigenvalues -- $-0.08573-0.05599-0.04656-0.04635-0.04412$ Alpha virt. eigenvalues -- $-0.02050-0.01731-0.01407-0.01379-0.01291$ Alpha virt. eigenvalues -- $-0.01255-0.00503-0.00347-0.000620 .01308$ Alpha virt. eigenvalues -- 0.024900 .025360 .036800 .049740 .05325 Alpha virt. eigenvalues -- 0.053750 .054460 .054670 .057460 .05840 Alpha virt. eigenvalues -- 0.062960 .073260 .078140 .082170 .08328 Alpha virt. eigenvalues -- 0.084470 .085090 .087530 .089890 .09206 Alpha virt. eigenvalues -- 0.092900 .093480 .096070 .100430 .10118 Alpha virt. eigenvalues -- 0.101450 .101500 .104030 .105540 .10640 Alpha virt. eigenvalues -- 0.108540 .110240 .112130 .114280 .11446 Alpha virt. eigenvalues -- 0.115300 .115350 .115550 .117540 .11835 Alpha virt. eigenvalues -- 0.118710 .119100 .119560 .121130 .12177 Alpha virt. eigenvalues -- 0.123770 .124060 .124270 .124950 .12573 Alpha virt. eigenvalues -- 0.127440 .128010 .129000 .132040 .13370 
Alpha virt. eigenvalues -- 0.134430 .136890 .138210 .138500 .14202 Alpha virt. eigenvalues -- 0.146460 .149220 .150220 .155190 .15646 Alpha virt. eigenvalues -- 0.159310 .161130 .162380 .164700 .16635 Alpha virt. eigenvalues -- 0.169910 .170820 .171700 .171730 .17283 Alpha virt. eigenvalues -- 0.173340 .174520 .175560 .176010 .17622 Alpha virt. eigenvalues -- 0.176650 .177050 .178470 .182030 .18285 Alpha virt. eigenvalues -- 0.184390 .185820 .187950 .188090 .18892 Alpha virt. eigenvalues -- 0.189320 .190300 .190400 .191040 .19210 Alpha virt. eigenvalues -- 0.192610 .193570 .194140 .197370 .19838 Alpha virt. eigenvalues -- 0.198860 .200680 .203730 .204890 .20671 Alpha virt. eigenvalues -- 0.208470 .208810 .210010 .211410 .21810 Alpha virt. eigenvalues -- 0.220110 .223510 .224710 .226790 .23165 Alpha virt. eigenvalues -- 0.234260 .239950 .240050 .240530 .24071 Alpha virt. eigenvalues -- 0.241540 .245150 .246770 .246830 .24727 Alpha virt. eigenvalues -- 0.247820 .250610 .250740 .253160 .25336 Alpha virt. eigenvalues -- 0.260510 .260900 .262940 .268500 .26967 Alpha virt. eigenvalues -- 0.272000 .272420 .279400 .282880 .28524 Alpha virt. eigenvalues -- 0.286060 .288250 .288960 .290390 .29473 Alpha virt. eigenvalues -- 0.297580 .300440 .303120 .306300 .30732 Alpha virt. eigenvalues -- 0.308990 .309260 .313150 .314870 .31960 Alpha virt. eigenvalues -- 0.320380 .324930 .326110 .328020 .33018 Alpha virt. eigenvalues -- 0.331690 .332790 .333350 .336000 .33692 Alpha virt. eigenvalues -- 0.337610 .342140 .346850 .348640 .35079 Alpha virt. eigenvalues -- 0.351000 .355710 .357540 .360980 .36462 Alpha virt. eigenvalues -- 0.366450 .371690 .373210 .374240 .37749 Alpha virt. eigenvalues -- 0.383070 .385380 .389400 .390720 .39420 Alpha virt. eigenvalues -- 0.397410 .399710 .402130 .405490 .40954 Alpha virt. eigenvalues -- 0.418750 .418770 .419200 .419260 .42233 Alpha virt. eigenvalues -- 0.422970 .423370 .425020 .432670 .43421 Alpha virt. eigenvalues -- 0.435900 .436970 .438220 .438340 .43884 Alpha virt. eigenvalues -- 0.440110 .440770 .442280 .442740 .44321 Alpha virt. eigenvalues -- 0.447250 .448120 .450930 .451390 .45441 Alpha virt. eigenvalues -- 0.455330 .456200 .457240 .459450 .46132 Alpha virt. eigenvalues -- 0.464390 .465860 .466570 .468040 .46893 Alpha virt. eigenvalues -- 0.469260 .470410 .473880 .475480 .47830 Alpha virt. eigenvalues -- 0.478680 .479660 .479940 .481500 .48238 Alpha virt. eigenvalues -- 0.485730 .488800 .489960 .490720 .49149 Alpha virt. eigenvalues -- 0.492510 .493830 .495400 .497250 .50031 Alpha virt. eigenvalues -- 0.501710 .502480 .505330 .506570 .50762 Alpha virt. eigenvalues -- 0.512930 .515110 .516500 .519780 .52006 Alpha virt. eigenvalues -- 0.524330 .526170 .530200 .532790 .53981 Alpha virt. eigenvalues -- 0.549820 .550970 .552610 .554350 .55723 Alpha virt. eigenvalues -- 0.558730 .559390 .561240 .563350 .56484 Alpha virt. eigenvalues -- 0.566790 .567440 .570420 .572760 .57562 Alpha virt. eigenvalues -- 0.576460 .577270 .578660 .583090 .58500 Alpha virt. eigenvalues -- 0.587130 .587370 .588380 .594150 .59582 Alpha virt. eigenvalues -- 0.596590 .598970 .600950 .602810 .60544 Alpha virt. eigenvalues -- 0.606350 .608710 .611670 .614850 .61578 Alpha virt. eigenvalues -- 0.618240 .618930 .620020 .620730 .62363 Alpha virt. eigenvalues -- 0.624150 .626040 .626890 .628910 .62972 Alpha virt. eigenvalues -- 0.633470 .636310 .638820 .640380 .64324 Alpha virt. eigenvalues -- 0.643690 .645630 .647280 .649320 .64993 Alpha virt. eigenvalues -- 0.652480 .652770 .654940 .661070 .66139 Alpha virt. eigenvalues -- 0.664870 .666430 .668150 .670150 .67033 Alpha virt. eigenvalues -- 0.670490 .671150 .671810 .673050 .67347 Alpha virt. eigenvalues -- 0.674280 .675220 .675550 .676460 .67724 
Alpha virt. eigenvalues -- 0.677660 .678000 .678460 .680350 .68138 Alpha virt. eigenvalues -- 0.682210 .683480 .684080 .685470 .68603 Alpha virt. eigenvalues -- 0.687550 .688040 .689010 .689880 .69050 Alpha virt. eigenvalues -- 0.691240 .691730 .693900 .694810 .69585 Alpha virt. eigenvalues -- 0.696200 .696820 .698480 .698650 .69906 Alpha virt. eigenvalues -- 0.700350 .701280 .702730 .704380 .70480 Alpha virt. eigenvalues -- 0.704920 .706030 .706200 .707510 .70980 Alpha virt. eigenvalues -- 0.710730 .711110 .712440 .714600 .71713 Alpha virt. eigenvalues -- 0.718230 .719710 .720460 .720750 .72157 Alpha virt. eigenvalues -- 0.722880 .723350 .723510 .725080 .72562 Alpha virt. eigenvalues -- 0.726410 .727220 .728430 .730840 .73115 Alpha virt. eigenvalues -- 0.731830 .732630 .734140 .734270 .73538 Alpha virt. eigenvalues -- 0.736010 .738240 .738930 .739090 .74083 Alpha virt. eigenvalues -- 0.742080 .742320 .744950 .745570 .74700 Alpha virt. eigenvalues -- 0.747750 .749360 .749650 .750670 .75161 Alpha virt. eigenvalues -- 0.752940 .753290 .754220 .756300 .75674 Alpha virt. eigenvalues -- 0.758140 .758990 .760200 .761860 .76246 Alpha virt. eigenvalues -- 0.763070 .763650 .767450 .769040 .77142 Alpha virt. eigenvalues -- 0.772070 .775310 .776480 .776860 .77771 Alpha virt. eigenvalues -- 0.780570 .781640 .784740 .785710 .78965 Alpha virt. eigenvalues -- 0.790810 .793660 .796550 .797430 .80015 Alpha virt. eigenvalues -- 0.800370 .801440 .802090 .803370 .80506 Alpha virt. eigenvalues -- 0.807580 .810000 .810620 .814360 .81470 Alpha virt. eigenvalues -- 0.815990 .817310 .819460 .823370 .82550 Alpha virt. eigenvalues -- 0.827170 .829050 .829370 .831450 .83553 Alpha virt. eigenvalues -- 0.838060 .840320 .842030 .842450 .84352 Alpha virt. eigenvalues -- 0.843570 .845000 .845520 .846160 .84667 Alpha virt. eigenvalues -- 0.848030 .849050 .849840 .850580 .85160 Alpha virt. eigenvalues -- 0.855120 .857440 .863720 .868540 .87053 Alpha virt. eigenvalues -- 0.871780 .872490 .875100 .876910 .88146 Alpha virt. eigenvalues -- 0.888590 .889990 .891500 .892200 .90057 Alpha virt. eigenvalues -- 0.909640 .910870 .912010 .914260 .93090 Alpha virt. eigenvalues -- 0.938730 .945960 .953520 .955430 .95891 Alpha virt. eigenvalues -- 0.960620 .974400 .977550 .994651 .00015 Alpha virt. eigenvalues -- 1.004811 .009481 .010111 .012621 .01724 Alpha virt. eigenvalues -- 1.018131 .026941 .030151 .031001 .03360 Alpha virt. eigenvalues -- 1.037251 .039171 .044851 .047591 .05011 Alpha virt. eigenvalues -- 1.058431 .063721 .071491 .079101 .08302 Alpha virt. eigenvalues -- 1.088701 .090511 .092211 .093051 .09764 Alpha virt. eigenvalues -- 1.100341 .100801 .102771 .103791 .10667 Alpha virt. eigenvalues -- 1.113191 .118831 .120471 .126671 .12753 Alpha virt. eigenvalues -- 1.128531 .131291 .134071 .135601 .13671 Alpha virt. eigenvalues -- 1.137051 .138101 .140051 .140741 .14205 Alpha virt. eigenvalues -- 1.144961 .148871 .155161 .165171 .17167 Alpha virt. eigenvalues -- 1.177491 .187141 .188101 .191761 .19227 Alpha virt. eigenvalues -- 1.197521 .216871 .224501 .241141 .24303 Alpha virt. eigenvalues -- 1.246871 .251161 .256771 .261441 .26340 Alpha virt. eigenvalues -- 1.273561 .290721 .302821 .303451 .30701 Alpha virt. eigenvalues -- 1.307261 .314341 .315191 .322921 .32662 Alpha virt. eigenvalues -- 1.332201 .334541 .341341 .350801 .35300 Alpha virt. eigenvalues -- 1.357721 .370601 .373191 .383681 .38842 Alpha virt. eigenvalues -- 1.392201 .415891 .424651 .435101 .43871 Alpha virt. eigenvalues -- 1.461211 .464141 .472781 .522031 .55975 Alpha virt. eigenvalues -- 1.566661 .576381 .584671 .584781 .59058 Alpha virt. eigenvalues -- 1.591271 .614191 .619401 .742961 .80759 Alpha virt. eigenvalues -- 1.826631 .89387 


\section{Chloroform}

Alpha occ. eigenvalues -- -19.21745 -19.21745 -14.34171 -14.34170 -10.29412 Alpha occ. eigenvalues -- -10.29412 -10.26072 -10.26061 -10.23582 -10.23573 Alpha occ. eigenvalues -- -10.22015 -10.22005 - 10.21282 -10.21282 -10.20400 Alpha occ. eigenvalues -- -10.20400 -10.20388 -10.20388 -10.19863 -10.19850 Alpha occ. eigenvalues -- -10.19704 -10.19704 -10.19685 -10.19685 -10.19601 Alpha occ. eigenvalues -- -10.19601 -10.19463 -10.19463 -10.19436 -10.19436 Alpha occ. eigenvalues -- -10.19382 -10.19382 -10.19288 -10.19288 - 10.19147 Alpha occ. eigenvalues -- -10.19147 -10.19006 -10.19006 -10.18981 -10.18981 Alpha occ. eigenvalues -- -10.18894 -10.18894 -10.18264 -10.18264 -10.18242 Alpha occ. eigenvalues -- -10.18242 -10.18217 - 10.18217 -10.18173 -10.18173 Alpha occ. eigenvalues -- -10.17978 -10.17977 -10.17940 -10.17940 - 10.17915 Alpha occ. eigenvalues -- -10.17915 -10.17864 -10.17864-10.17828 -10.17828 Alpha occ. eigenvalues -- -10.17796 -10.17795 -10.17607 -10.17607 -10.17600 Alpha occ. eigenvalues -- -10.17600 -10.15915 - 10.15915 -10.15913 -10.15913 Alpha occ. eigenvalues -- -10.15901 -10.15901 -10.15896-10.15896-10.15825 Alpha occ. eigenvalues -- -10.15825 -10.15824 -10.15824 -10.15801 -10.15801 Alpha occ. eigenvalues -- -10.15778 -10.15778 -10.15716 -10.15716 -1.14939 Alpha occ. eigenvalues -- -1.14834 -0.95656 -0.95649 -0.90122 -0.89365 Alpha occ. eigenvalues -- -0.88806 -0.87384 -0.87383-0.87246 -0.86754 Alpha occ. eigenvalues -- -0.86684 -0.86263-0.83052 -0.82847 -0.82842 Alpha occ. eigenvalues -- $-0.82742-0.82381-0.81910-0.80807-0.79470$ Alpha occ. eigenvalues -- $-0.79380-0.78649-0.78623-0.75874-0.75334$ Alpha occ. eigenvalues -- $-0.75270-0.75264-0.75231-0.75191-0.75014$ Alpha occ. eigenvalues -- $-0.74887-0.73722-0.73116-0.72645-0.72550$ Alpha occ. eigenvalues -- -0.72255 -0.69535-0.68516-0.68483-0.68478 Alpha occ. eigenvalues -- -0.68458 -0.68423-0.68394-0.68298 -0.68298 Alpha occ. eigenvalues -- -0.68280 -0.68280 -0.68206-0.68206 -0.66680 Alpha occ. eigenvalues -- -0.65389 -0.65261 -0.65058 -0.64159-0.63655 Alpha occ. eigenvalues -- -0.62856 -0.62143 -0.61618 -0.61292 -0.61203 Alpha occ. eigenvalues -- -0.61185 -0.60636 -0.60426 -0.59793-0.59296 Alpha occ. eigenvalues -- -0.59019 -0.58672 -0.58293-0.56653-0.55990 Alpha occ. eigenvalues -- -0.55726 -0.55624 -0.55621 -0.55592 -0.55435 Alpha occ. eigenvalues -- $-0.55384-0.54683-0.52436-0.51941-0.51830$ Alpha occ. eigenvalues -- $-0.50850-0.50511-0.50453-0.50323-0.50251$ Alpha occ. eigenvalues -- -0.50197 -0.49593 -0.49369-0.48873-0.48215 Alpha occ. eigenvalues -- -0.47833 -0.47829 -0.47439 -0.47439-0.47057 Alpha occ. eigenvalues -- -0.46833 -0.46383 -0.46047-0.45932 -0.45916 Alpha occ. eigenvalues -- $-0.45913-0.45427-0.45421-0.45186-0.45043$ Alpha occ. eigenvalues -- -0.45021 -0.44931 -0.44868 -0.44721 -0.44534 Alpha occ. eigenvalues -- $-0.44533-0.44515-0.44513-0.44438-0.44435$ Alpha occ. eigenvalues -- -0.43340 -0.43335 -0.43317-0.43308 -0.43022 Alpha occ. eigenvalues -- $-0.43010-0.42900-0.42662-0.42645-0.42622$ Alpha occ. eigenvalues -- -0.42621 -0.42456 -0.41764-0.41603-0.40994 Alpha occ. eigenvalues -- -0.40553 -0.40421 -0.40353-0.40254-0.40148 Alpha occ. eigenvalues -- -0.40088 -0.40074-0.40065 -0.40050 -0.39807 Alpha occ. eigenvalues -- $-0.39724-0.39547-0.38782-0.38596-0.38243$ Alpha occ. eigenvalues -- -0.38176 -0.38157 -0.38060 -0.38020 -0.37871 Alpha occ. eigenvalues -- $-0.37867-0.37834-0.37517-0.37475-0.37420$ Alpha occ. eigenvalues -- $-0.37025-0.36997-0.36812-0.36777-0.36774$ Alpha occ. eigenvalues -- -0.36764-0.36747 -0.36743-0.36654-0.36648 Alpha occ. eigenvalues -- $-0.36195-0.35967-0.35744-0.35669-0.35621$ Alpha occ. eigenvalues -- $-0.35609-0.35600-0.35550-0.34643-0.33875$ Alpha occ. eigenvalues -- -0.33874 -0.33764 -0.33680 -0.33646-0.33456 
Alpha occ. eigenvalues -- -0.33264 -0.33259 -0.33236 -0.33168 -0.33079 Alpha occ. eigenvalues -- $-0.33075-0.32387-0.32369-0.32334-0.32333$ Alpha occ. eigenvalues -- $-0.32241-0.32191-0.30895-0.30486-0.29980$ Alpha occ. eigenvalues -- $-0.29754-0.29733-0.28860-0.28494-0.26710$ Alpha occ. eigenvalues -- $-0.26703-0.26676-0.26672-0.26405-0.26336$ Alpha occ. eigenvalues -- $-0.25743-0.25401-0.24437-0.23720-0.23691$ Alpha occ. eigenvalues -- $-0.23688-0.21980$

Alpha virt. eigenvalues -- -0.09408 -0.06376 -0.05302 -0.05267 -0.05233 Alpha virt. eigenvalues -- -0.02611 -0.02269-0.01798 -0.01790 -0.01752 Alpha virt. eigenvalues -- $-0.01736-0.01467-0.01342-0.010730 .00428$ Alpha virt. eigenvalues -- 0.019840 .020090 .026990 .043300 .05271 Alpha virt. eigenvalues -- 0.054580 .054860 .057360 .057790 .05807 Alpha virt. eigenvalues -- 0.058890 .066490 .069370 .078740 .07938 Alpha virt. eigenvalues -- 0.083340 .084390 .089470 .089620 .09216 Alpha virt. eigenvalues -- 0.095370 .098070 .098590 .102390 .10239 Alpha virt. eigenvalues -- 0.104040 .104210 .105590 .106570 .10790 Alpha virt. eigenvalues -- 0.108070 .109310 .110940 .113690 .11409 Alpha virt. eigenvalues -- 0.115440 .115950 .116740 .116750 .11712 Alpha virt. eigenvalues -- 0.117820 .118580 .118920 .119460 .12039 Alpha virt. eigenvalues -- 0.122230 .126030 .126210 .128250 .12842 Alpha virt. eigenvalues -- 0.128920 .129660 .129900 .130970 .13279 Alpha virt. eigenvalues -- 0.135270 .136160 .136450 .139370 .14006 Alpha virt. eigenvalues -- 0.142090 .144790 .149090 .149660 .15564 Alpha virt. eigenvalues -- 0.157830 .161240 .162660 .163550 .16492 Alpha virt. eigenvalues -- 0.167850 .172070 .172820 .173350 .17409 Alpha virt. eigenvalues -- 0.174510 .175470 .175780 .176040 .17610 Alpha virt. eigenvalues -- 0.176860 .177530 .178630 .179080 .18300 Alpha virt. eigenvalues -- 0.183450 .183850 .186220 .188160 .18881 Alpha virt. eigenvalues -- 0.190490 .190510 .191440 .191600 .19202 Alpha virt. eigenvalues -- 0.192080 .193110 .193370 .193990 .19444 Alpha virt. eigenvalues -- 0.195910 .195980 .198820 .201900 .20240 Alpha virt. eigenvalues -- 0.204730 .205640 .207000 .207580 .21653 Alpha virt. eigenvalues -- 0.220520 .221000 .221470 .221760 .22567 Alpha virt. eigenvalues -- 0.226120 .233400 .238230 .238320 .23905 Alpha virt. eigenvalues -- 0.239190 .240080 .244490 .244770 .24711 Alpha virt. eigenvalues -- 0.247210 .247240 .247260 .249260 .24964 Alpha virt. eigenvalues -- 0.254610 .258420 .259690 .265150 .26654 Alpha virt. eigenvalues -- 0.266600 .267410 .269830 .274120 .27710 Alpha virt. eigenvalues -- 0.278750 .279950 .281500 .284060 .28962 Alpha virt. eigenvalues -- 0.291390 .294550 .296640 .298210 .30144 Alpha virt. eigenvalues -- 0.303340 .306200 .306530 .307010 .31061 Alpha virt. eigenvalues -- 0.311080 .316270 .317990 .320140 .32100 Alpha virt. eigenvalues -- 0.323910 .325950 .327080 .329790 .33273 Alpha virt. eigenvalues -- 0.333380 .337920 .340210 .343620 .34435 Alpha virt. eigenvalues -- 0.344710 .349880 .350080 .353430 .35547 Alpha virt. eigenvalues -- 0.358000 .365010 .366150 .367120 .37185 Alpha virt. eigenvalues -- 0.377080 .378960 .383210 .384290 .38923 Alpha virt. eigenvalues -- 0.390110 .394600 .395170 .400420 .40273 Alpha virt. eigenvalues -- 0.414330 .414370 .420510 .420520 .42073 Alpha virt. eigenvalues -- 0.420760 .421490 .421500 .426080 .42972 Alpha virt. eigenvalues -- 0.431060 .431080 .433750 .434690 .43485 Alpha virt. eigenvalues -- 0.436570 .436650 .437660 .439800 .44001 Alpha virt. eigenvalues -- 0.440450 .441620 .444560 .446450 .44788 Alpha virt. eigenvalues -- 0.450490 .452590 .455240 .455730 .45658 Alpha virt. eigenvalues -- 0.459990 .461140 .462340 .462470 .46329 Alpha virt. eigenvalues -- 0.463440 .464020 .469200 .470380 .47071 
Alpha virt. eigenvalues -- 0.473210 .474090 .474740 .478240 .47885 Alpha virt. eigenvalues -- 0.480170 .481100 .483310 .484540 .48839 Alpha virt. eigenvalues -- 0.489650 .490080 .491020 .492090 .49622 Alpha virt. eigenvalues -- 0.496870 .497220 .501000 .501260 .50265 Alpha virt. eigenvalues -- 0.507860 .509200 .511950 .514230 .51517 Alpha virt. eigenvalues -- 0.516600 .519430 .523370 .523760 .53468 Alpha virt. eigenvalues -- 0.539090 .544210 .544970 .549220 .55075 Alpha virt. eigenvalues -- 0.553500 .554200 .557970 .557980 .56233 Alpha virt. eigenvalues -- 0.562580 .564030 .566290 .571710 .57220 Alpha virt. eigenvalues -- 0.573140 .573930 .576880 .577230 .57951 Alpha virt. eigenvalues -- 0.580490 .582180 .585370 .586710 .58777 Alpha virt. eigenvalues -- 0.593270 .593630 .594780 .599280 .60152 Alpha virt. eigenvalues -- 0.601690 .603110 .609350 .610020 .61463 Alpha virt. eigenvalues -- 0.615820 .616460 .618980 .619230 .62056 Alpha virt. eigenvalues -- 0.621120 .621270 .622420 .624570 .62580 Alpha virt. eigenvalues -- 0.629860 .629990 .632130 .632270 .63708 Alpha virt. eigenvalues -- 0.637910 .642590 .643910 .646570 .64857 Alpha virt. eigenvalues -- 0.649940 .650370 .650550 .652970 .65675 Alpha virt. eigenvalues -- 0.659900 .661650 .665250 .667450 .66829 Alpha virt. eigenvalues -- 0.668350 .670150 .670680 .670860 .67135 Alpha virt. eigenvalues -- 0.672640 .673090 .673590 .674270 .67479 Alpha virt. eigenvalues -- 0.675640 .676150 .677760 .678120 .67854 Alpha virt. eigenvalues -- 0.679170 .680690 .683400 .684210 .68493 Alpha virt. eigenvalues -- 0.685450 .686220 .686480 .686650 .68880 Alpha virt. eigenvalues -- 0.689190 .690770 .690950 .691650 .69220 Alpha virt. eigenvalues -- 0.692990 .693800 .695400 .697750 .69787 Alpha virt. eigenvalues -- 0.699080 .699820 .700880 .701360 .70206 Alpha virt. eigenvalues -- 0.702390 .704240 .704960 .705160 .70614 Alpha virt. eigenvalues -- 0.708750 .709360 .711630 .711980 .71223 Alpha virt. eigenvalues -- 0.715020 .715880 .719160 .719310 .72091 Alpha virt. eigenvalues -- 0.721780 .722560 .722670 .725160 .72586 Alpha virt. eigenvalues -- 0.726000 .726140 .726450 .727580 .72759 Alpha virt. eigenvalues -- 0.729690 .731340 .731690 .732780 .73298 Alpha virt. eigenvalues -- 0.734880 .734920 .735270 .735730 .73815 Alpha virt. eigenvalues -- 0.739130 .741950 .742670 .743340 .74380 Alpha virt. eigenvalues -- 0.745220 .745700 .747130 .748800 .74905 Alpha virt. eigenvalues -- 0.751600 .752540 .753090 .753160 .75455 Alpha virt. eigenvalues -- 0.755510 .756860 .757360 .759980 .76094 Alpha virt. eigenvalues -- 0.761510 .763800 .764680 .765210 .76602 Alpha virt. eigenvalues -- 0.766470 .769470 .771460 .772540 .77456 Alpha virt. eigenvalues -- 0.775680 .777110 .778370 .778840 .78024 Alpha virt. eigenvalues -- 0.783050 .786560 .791070 .793740 .79510 Alpha virt. eigenvalues -- 0.796400 .796660 .799460 .799650 .80219 Alpha virt. eigenvalues -- 0.803970 .805020 .805080 .811200 .81297 Alpha virt. eigenvalues -- 0.813640 .814420 .815070 .816300 .81971 Alpha virt. eigenvalues -- 0.823730 .827000 .827910 .830210 .83024 Alpha virt. eigenvalues -- 0.832080 .834920 .835350 .836950 .83892 Alpha virt. eigenvalues -- 0.840740 .843740 .843840 .844520 .84572 Alpha virt. eigenvalues -- 0.846350 .846970 .848150 .848210 .84827 Alpha virt. eigenvalues -- 0.848820 .852300 .853100 .861570 .86367 Alpha virt. eigenvalues -- 0.865240 .866530 .870630 .871570 .87622 Alpha virt. eigenvalues -- 0.879110 .880590 .884950 .888020 .89737 Alpha virt. eigenvalues -- 0.902160 .907460 .908680 .908730 .92203 Alpha virt. eigenvalues -- 0.933960 .938390 .940760 .941890 .94949 Alpha virt. eigenvalues -- 0.954500 .962600 .970430 .982470 .98762 Alpha virt. eigenvalues -- 0.990031 .001861 .007691 .010711 .01677 
Alpha virt. eigenvalues -- 1.016861 .023581 .024221 .030041 .03015 Alpha virt. eigenvalues -- 1.037601 .039571 .041411 .046281 .04828 Alpha virt. eigenvalues -- 1.053591 .058901 .060211 .073701 .07513 Alpha virt. eigenvalues -- 1.077681 .086061 .089221 .089611 .09382 Alpha virt. eigenvalues -- 1.093901 .097411 .098861 .101961 .10234 Alpha virt. eigenvalues -- 1.105631 .114661 .116191 .123221 .12332 Alpha virt. eigenvalues -- 1.124551 .126521 .127431 .130201 .13323 Alpha virt. eigenvalues -- 1.134371 .134551 .138151 .139041 .14099 Alpha virt. eigenvalues -- 1.144081 .150831 .151551 .154851 .15575 Alpha virt. eigenvalues -- 1.168401 .175781 .176441 .182901 .18459 Alpha virt. eigenvalues -- 1.184811 .187861 .210421 .218091 .23156 Alpha virt. eigenvalues -- 1.234341 .247001 .250171 .253141 .25853 Alpha virt. eigenvalues -- 1.270511 .284221 .288321 .291731 .29941 Alpha virt. eigenvalues -- 1.301941 .307771 .315361 .319761 .32357 Alpha virt. eigenvalues -- 1.330741 .332981 .333601 .345931 .35039 Alpha virt. eigenvalues -- 1.351491 .357481 .364221 .374691 .38122 Alpha virt. eigenvalues -- 1.383031 .407891 .418541 .427331 .43137 Alpha virt. eigenvalues -- 1.452491 .453981 .463301 .514421 .55009 Alpha virt. eigenvalues -- 1.557991 .569591 .582931 .583591 .58662 Alpha virt. eigenvalues -- 1.587791 .602741 .610931 .730021 .79994 Alpha virt. eigenvalues -- 1.817591 .88240

\section{Comparison of Experimental and DFT UV-Vis}

\section{Gas Phase}

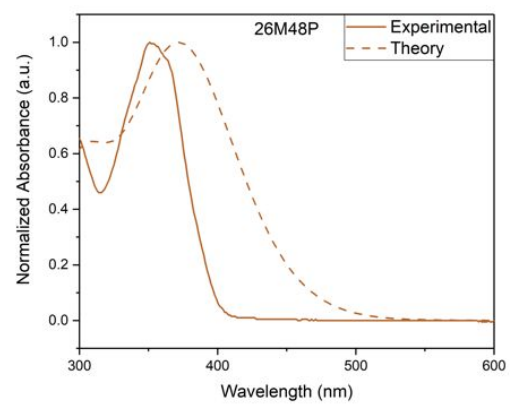

Chloroform

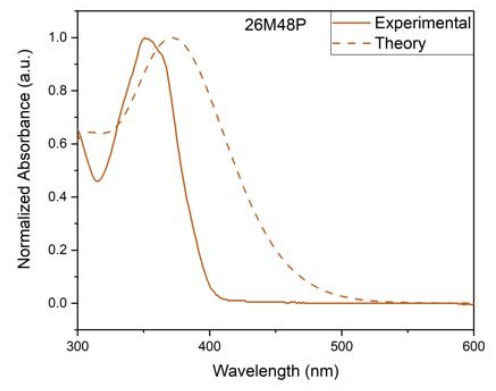

Figure S30. Gas phase and chloroform overlays of computation and experimental data for 26M48P.

\section{Lowest 15 Excited States}

\begin{tabular}{|c|c|c|c|c|c|c|}
\hline \multicolumn{4}{|c|}{ Gas Phase } & \multicolumn{3}{|c|}{ Chloroform } \\
\hline$\frac{\text { Excited }}{\text { state }}$ & $\frac{\text { Energy }}{(\mathrm{eV})}$ & $\frac{\text { Wavelength }}{(\mathrm{nm})}$ & $f$ & $\frac{\text { Energy }}{(\mathrm{eV})}$ & $\frac{\text { Wavelength }}{(\mathrm{nm})}$ & $f$ \\
\hline 1 & 3.05 & $\overline{407.05}$ & 0.54 & 3.02 & $\overline{407.54}$ & 0.75 \\
\hline 2 & 3.49 & 355.31 & 1.13 & 3.43 & 361.40 & 1.14 \\
\hline 3 & 3.56 & 348.55 & 0.00 & 3.47 & 357.17 & 0.00 \\
\hline 4 & 3.57 & 348.30 & 0.06 & 3.47 & 356.94 & 0.09 \\
\hline 5 & 3.70 & 335.16 & 0.00 & 3.72 & 333.72 & 0.00 \\
\hline 6 & 3.80 & 326.41 & 0.00 & 3.77 & 329.28 & 0.00 \\
\hline 7 & 3.98 & 311.26 & 0.00 & 3.92 & 316.47 & 0.22 \\
\hline 8 & 3.99 & 310.42 & 0.06 & 3.95 & 313.58 & 0.00 \\
\hline 9 & 4.04 & 307.09 & 0.01 & 4.07 & 304.88 & 0.90 \\
\hline
\end{tabular}




\begin{tabular}{|l|lll|l|lll|}
10 & 4.04 & 306.52 & 0.18 & 4.08 & 303.95 & 0.02 \\
11 & 4.05 & 306.21 & 0.00 & 4.10 & 302.35 & 0.00 \\
12 & 4.05 & 305.95 & 0.00 & 4.13 & 300.23 & 0.00 \\
13 & 4.10 & 302.34 & 0.84 & 4.13 & 300.23 & 0.12 \\
14 & 4.30 & 288.62 & 0.01 & 4.26 & 290.73 & 0.10 \\
15 & 4.30 & 288.47 & 0.00 & 4.27 & 290.58 & 0.00 \\
\hline
\end{tabular}

Table S7. Gas phase and chloroform excitation energies, wavelengths, and oscillator strengths for first 15 excited state of 26M48P.

\section{Geometric Images}

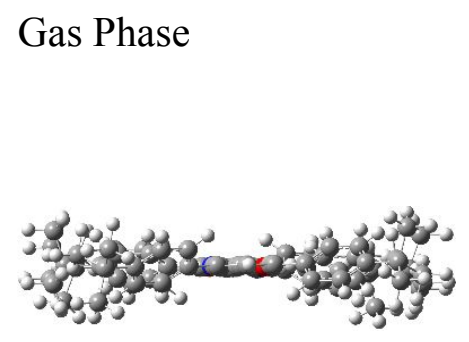

\section{Chloroform}
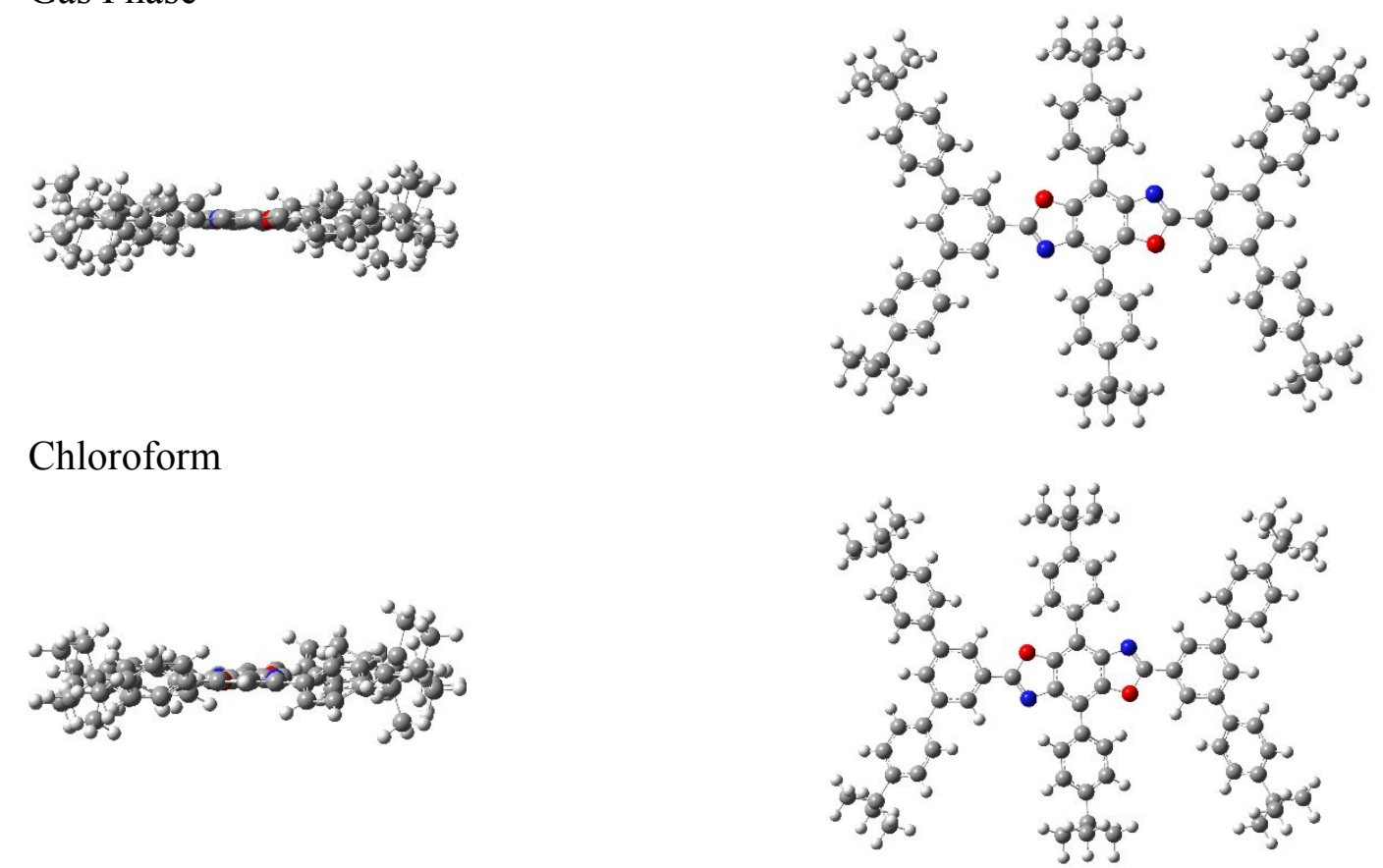

Figure S31. Geometric optimization images in gas phase and chloroform for 26M48P.

\section{P48P}

\section{Cartesian Coordinates}

Gas Phase

C -0.1924 -1.5014 -0.03401

C - $1.222-0.54972-0.0364$

C $0.192411 .50139-0.0341$

C $1.222010 .54971-0.03645$

C - $1.074740 .86499-0.02753$

C -3.21244 0.45258 -0.01098

O $-2.59489-0.80714-0.02561$

$\mathrm{N}-2.351841 .44069-0.01349$

C $1.07475-0.86499-0.02748$

C $3.21244-0.45259-0.01098$

$\mathrm{N} 2.35184-1.4407-0.01343$

O $2.59490 .80713-0.02568$

C $0.400872 .96264-0.03411$ 
C $1.590793 .53468-0.53212$

C -0.583073 .845690 .46741$

C $1.788364 .92129-0.52709$

H $2.372292 .89052-0.9389$

C -0.371945 .226020 .4755$

H - 1.52363 .439590 .8428

C $0.816525 .8039-0.02004$

H $2.725915 .30716-0.93327$

H - 1.161235 .86650 .88172

C - $0.40087-2.96264-0.03392$

C - $1.59079-3.53472-0.53188$

C $0.58307-3.845660 .46765$

C $-1.78836-4.92133-0.52677$

H -2.37229 - $2.89057-0.93871$

C $0.37195-5.225990 .47583$

H $1.52361-3.439530 .84301$

C $-0.81652-5.80391-0.01966$

H - $2.72592-5.30722-0.93292$

H $1.16124-5.866450 .88209$

C 1.002717 .330670 .01071

C - $0.125948 .00374-0.81039$

C 0.936057 .824741 .47794

C $2.35777 .76305-0.58459$

H - $0.102917 .66317-1.86259$

H - $1.125557 .77-0.40038$

H - $0.0049 .10431-0.79922$

H 1.73537 .362 .08529

H 1.063588 .924011 .51946

H -0.033587 .577621 .94775$

H $2.448448 .86494-0.5433$

H $3.207377 .33664-0.01939$

H $2.455377 .45862-1.64336$

C $-1.00272-7.330670 .01119$

C $0.12592-8.0038-0.80987$

C $-0.93604-7.824641 .47845$

C $-2.35771-7.76308-0.58407$

H $0.10288-7.6633-1.8621$

H $1.12554-7.77003-0.39989$

H $0.00399-9.10437-0.79862$

$\mathrm{H}-1.73528-7.359862 .08578$

$\mathrm{H}-1.06357-8.92391 .52005$

H $0.03359-7.577481 .94824$

H $-2.44845-8.86498-0.5427$

H $-3.20738-7.33664-0.01889$

H -2.45539 -7.45873 - 1.64286

C $-4.661440 .5057-0.00105$

C $-5.43974-0.666820 .08119$

C $-5.315341 .74991-0.0734$

C -6.83335 -0.58516 0.08933

H -4.94392 - 1.639040 .14428

C $-6.712121 .81785-0.06573$

H $-4.713042 .66008-0.14041$

C -7.505770 .655150 .01524$

$\mathrm{H}-7.40825-1.513730 .15662$

H -7.18168 2.80156 -0.12647

C $4.66144-0.5057-0.00107$ 
C 5.439740 .666830 .0811

C $5.31535-1.74992-0.07336$

C 6.833350 .585160 .08922

H 4.943921 .639040 .14414

C $6.71213-1.81786-0.0657$

H $4.71304-2.66009-0.14031$

C $7.50577-0.655140 .0152$

H 7.40826 1.51374 0.15646

H $7.18168-2.80156-0.12639$

C -9.043620 .693630 .02503$

C -9.58411 - $0.10857-1.18578$

C -9.563640 .057031 .33893$

C $-9.592 .13248-0.06459$

H $-9.226260 .32637-2.13778$

H -9.26358 -1.16597-1.1535

H - 10.69107 -0.09093 -1.19194

H -9.19062 0.61228 2.21989

H - 10.670340 .077161 .36049

H -9.24277 -0.99582 1.4402

H -10.69576 2.11083 -0.05471

H -9.260742 .749960 .79175$

H $-9.274262 .63299-0.9988$

C $9.04362-0.693630 .02498$

C $9.58410 .10852-1.18587$

C $9.56366-0.056971 .33885$

C $9.59-2.13248-0.06458$

H $9.22624-0.32646-2.13785$

H $9.263581 .16593-1.15363$

H $10.691070 .09087-1.19204$

H $9.19064-0.612182 .21983$

H $10.67036-0.07711 .3604$

H 9.242780 .995881 .44007

H $10.69576-2.11083-0.05471$

H $9.26075-2.749920 .79179$

H $9.27425-2.63303-0.99877$

\section{Chloroform}

C $1.22707-0.53892-0.04104$

C $-0.204191 .49688-0.03532$

C - $1.227070 .53892-0.04102$

C - $1.06883-0.8737-0.03388$

C $0.20419-1.49688-0.03532$

$\mathrm{N}-2.34153-1.4599-0.02807$

C -3.21091 $-0.47735-0.02545$

O -2.60103 $0.78663-0.03196$

C $3.210910 .47735-0.0255$

C $1.068830 .8737-0.0339$

O $2.60103-0.78663-0.032$

N $2.341531 .4599-0.02811$

C $4.659480 .5382-0.02251$

C $5.44333-0.633710 .02451$

C $5.309211 .78693-0.06667$

C $6.83697-0.547210 .02632$

H $4.95494-1.61090 .06262$

C $6.706081 .86008-0.06559$ 
H $4.708572 .69978-0.10486$ C $7.504640 .69796-0.01942$ H $7.41417-1.475720 .06514$ H $7.170672 .84717-0.1027$ C $-4.65948-0.5382-0.02243$ C -5.443330 .633710 .02459$ C $-5.30921-1.78693-0.06657$ C -6.836970 .547210 .02643$ H -4.954941 .61090 .06269$ C -6.70608 - $1.86008-0.06548$ $\mathrm{H}-4.70857-2.69978-0.10478$ C $-7.50464-0.69796-0.0193$ H -7.41417 1.475730 .06525 H -7.17067 -2.84717 -0.10257 C $-9.04184-0.74284-0.01659$ C -9.57012 -0.07718 1.27975 H -9.25440 .979311 .35698$ H - $10.67665-0.101861 .29445$ H -9.20124 -0.61199 2.17519 C $-9.58152-2.18571-0.07583$ H $-9.25512-2.782050 .79644$ H - $10.68722-2.16643-0.07275$ H $-9.25853-2.70672-0.99628$ C $-9.578670 .03009-1.24818$ H $-9.265311 .09011-1.23647$ H $-9.21442-0.4252-2.18836$ H $-10.685250 .00478-1.25862$ C $9.041840 .74284-0.01674$ C 9.570140 .077191 .27959 H $9.25442-0.97931 .35683$ H 10.676670 .101861 .29428 H 9.20128 0.6122 .17504 C $9.57864-0.0301-1.24833$ H $9.214380 .42519-2.18851$ H $10.68523-0.00479-1.25879$ H $9.26529-1.09012-1.23662$ C $9.581522 .18571-0.076$ H $9.258512 .70671-0.99645$ H 9.255132 .782050 .79627 H $10.687222 .16643-0.07294$ C - $0.425992 .95634-0.02424$ C 0.51783 .837960 .55191 C $-1.590993 .52592-0.58095$ C 0.293975 .217030 .57207 H 1.43593 .434180 .98227 C $-1.803594 .91103-0.56049$ H - $-2.340832 .88284-1.04625$ C -0.87095 .79290 .01887$ H 1.051935 .85681 .03484 H -2.72053 5.29575 -1.01216 C -1.073397 .317050 .06414$ C $0.078678 .00967-0.70725$

C -1.062567 .793431 .53882$

C $-2.410797 .7442-0.57291$ H - $0.051879 .10876-0.68074$ H $1.065067 .77495-0.26653$ 
H $0.091657 .68874-1.76602$

H - 1.204838 .890371 .58565

H - 1.878557 .314912 .11274

H -0.106417 .555062 .04008$

H - $2.466327 .45955-1.64016$

H -3.2751 7.29708 - 0.04728

H - $2.515268 .84391-0.51419$

C $0.42599-2.95635-0.02424$

C $-0.5178-3.837960 .55192$

C $1.59098-3.52592-0.58097$

C $-0.29396-5.217030 .57208$

H - $1.43589-3.434180 .98229$

C $1.80359-4.91103-0.56051$

H $2.34082-2.88285-1.04627$

C $0.8709-5.79290 .01886$

H - $1.05192-5.856791 .03486$

H 2.72052 -5.29575 -1.01219

C $1.07339-7.317050 .06413$

C $-0.07867-8.00967-0.70724$

C $1.06258-7.793431 .53881$

C $2.41078-7.7442-0.57292$

H $0.05187-9.10876-0.68074$

H - $1.06506-7.77495-0.26652$

$\mathrm{H}-0.09166-7.68874-1.76601$

H $1.20484-8.890371 .58564$

H $1.87856-7.314912 .11273$

H $0.10643-7.555072 .04009$

H $2.4663-7.45955-1.64017$

H $3.2751-7.29708-0.0473$

H $2.51526-8.84391-0.5142$

\section{Energy Levels}

Gas Phase

Alpha occ. eigenvalues -- -19.20659 -19.20658 -14.32986 -14.32985 -10.28275

Alpha occ. eigenvalues -- -10.28274 -10.24997 -10.24986 -10.22484 -10.22474

Alpha occ. eigenvalues -- -10.20932 -10.20922 -10.20251 -10.20251 -10.20187

Alpha occ. eigenvalues -- -10.20187 -10.19633 -10.19633-10.19275 -10.19275

Alpha occ. eigenvalues -- -10.18845 -10.18845 -10.18756 -10.18744 -10.18663

Alpha occ. eigenvalues -- -10.18663 -10.18325 -10.18325 -10.18200 -10.18200

Alpha occ. eigenvalues -- -10.18017 -10.18017 -10.16934 -10.16933 -10.16728

Alpha occ. eigenvalues -- $-10.16728-10.16687-10.16687-10.16600-10.16600$

Alpha occ. eigenvalues -- $-10.16470-10.16470-10.16463-10.16463-10.16410$

Alpha occ. eigenvalues -- -10.16410 - 10.15649 -10.15649 -10.15639 -10.15639

Alpha occ. eigenvalues -- - $10.15628-10.15628-1.13821-1.13716-0.94557$

Alpha occ. eigenvalues -- $-0.94548-0.88928-0.87869-0.87323-0.86295$

Alpha occ. eigenvalues -- -0.85616 -0.83340 -0.82902 -0.82375 -0.81390

Alpha occ. eigenvalues -- $-0.79075-0.78512-0.76860-0.75757-0.75722$

Alpha occ. eigenvalues -- $-0.74252-0.74115-0.74047-0.73151-0.71524$

Alpha occ. eigenvalues -- $-0.69096-0.69072-0.68837-0.68837-0.68412$

Alpha occ. eigenvalues -- -0.68222 -0.68202 -0.67994-0.67994 -0.65452

Alpha occ. eigenvalues -- -0.64297 -0.64221 -0.62474-0.61090 -0.61004

Alpha occ. eigenvalues -- $-0.60825-0.60725-0.59184-0.58412-0.58385$

Alpha occ. eigenvalues -- $-0.56172-0.56019-0.55991-0.55168-0.54861$

Alpha occ. eigenvalues -- $-0.54723-0.52958-0.51666-0.50854-0.49764$

Alpha occ. eigenvalues -- $-0.49713-0.48916-0.48348-0.47863-0.47625$ 
Alpha occ. eigenvalues -- -0.47386 -0.47008 -0.46907 -0.46568 -0.45917 Alpha occ. eigenvalues -- $-0.45545-0.45101-0.45098-0.45061-0.44390$ Alpha occ. eigenvalues -- $-0.44367-0.44231-0.44224-0.43904-0.43822$ Alpha occ. eigenvalues -- $-0.43801-0.43041-0.43013-0.42452-0.42434$ Alpha occ. eigenvalues -- $-0.42071-0.41802-0.40983-0.40662-0.40559$ Alpha occ. eigenvalues -- $-0.40434-0.40343-0.39925-0.39713-0.39646$ Alpha occ. eigenvalues -- $-0.39119-0.38882-0.38562-0.38544-0.38361$ Alpha occ. eigenvalues -- $-0.37911-0.37771-0.37456-0.37438-0.37292$ Alpha occ. eigenvalues -- -0.37242 -0.36820 -0.36549-0.36545 -0.36540 Alpha occ. eigenvalues -- $-0.36256-0.35354-0.35180-0.34888-0.34884$ Alpha occ. eigenvalues -- $-0.34569-0.34305-0.33826-0.33812-0.33458$ Alpha occ. eigenvalues -- $-0.33294-0.32952-0.32939-0.32882-0.32768$ Alpha occ. eigenvalues -- $-0.32608-0.31781-0.31724-0.29172-0.28944$ Alpha occ. eigenvalues -- $-0.28640-0.27981-0.27031-0.26966-0.26318$ Alpha occ. eigenvalues -- $-0.25304-0.25245-0.24438-0.22891-0.20813$ Alpha virt. eigenvalues -- -0.08372 -0.05113 -0.04108 -0.01878 -0.01811 Alpha virt. eigenvalues -- $-0.00463-0.00359-0.000500 .011040 .03488$ Alpha virt. eigenvalues -- 0.043590 .050500 .050930 .057740 .05856 Alpha virt. eigenvalues -- 0.061070 .072690 .078000 .085740 .08634 Alpha virt. eigenvalues -- 0.092010 .093930 .096130 .096440 .10088 Alpha virt. eigenvalues -- 0.101320 .104770 .105680 .112410 .11246 Alpha virt. eigenvalues -- 0.113650 .113800 .118700 .119440 .12079 Alpha virt. eigenvalues -- 0.121400 .121500 .121990 .125270 .12652 Alpha virt. eigenvalues -- 0.128220 .128730 .132530 .143410 .14507 Alpha virt. eigenvalues -- 0.146780 .146970 .148900 .149320 .15290 Alpha virt. eigenvalues -- 0.160530 .164870 .165900 .169010 .16979 Alpha virt. eigenvalues -- 0.169850 .173220 .174110 .176490 .18063 Alpha virt. eigenvalues -- 0.183220 .183710 .185090 .185500 .18586 Alpha virt. eigenvalues -- 0.186630 .189350 .191800 .191930 .19278 Alpha virt. eigenvalues -- 0.193750 .197750 .199780 .201800 .20280 Alpha virt. eigenvalues -- 0.208770 .209660 .209790 .219180 .22137 Alpha virt. eigenvalues -- 0.229200 .233010 .233350 .239460 .24111 Alpha virt. eigenvalues -- 0.242820 .243020 .244280 .246290 .25121 Alpha virt. eigenvalues -- 0.251690 .253730 .254300 .269640 .27453 Alpha virt. eigenvalues -- 0.278680 .286420 .286420 .287440 .29067 Alpha virt. eigenvalues -- 0.296530 .297310 .304210 .307410 .31333 Alpha virt. eigenvalues -- 0.320520 .321230 .326600 .330170 .33354 Alpha virt. eigenvalues -- 0.336580 .339300 .346390 .348290 .35030 Alpha virt. eigenvalues -- 0.360500 .361160 .364590 .368860 .38542 Alpha virt. eigenvalues -- 0.387550 .392710 .397340 .407150 .41361 Alpha virt. eigenvalues -- 0.415260 .415270 .421810 .422400 .42320 Alpha virt. eigenvalues -- 0.423200 .425470 .426730 .433770 .43633 Alpha virt. eigenvalues -- 0.437850 .441100 .443040 .446690 .44915 Alpha virt. eigenvalues -- 0.453870 .454490 .455030 .458410 .46058 Alpha virt. eigenvalues -- 0.462340 .467020 .471060 .471590 .47554 Alpha virt. eigenvalues -- 0.477290 .478930 .481720 .482610 .48757 Alpha virt. eigenvalues -- 0.489170 .490360 .497140 .497900 .50220 Alpha virt. eigenvalues -- 0.503180 .504360 .510390 .517650 .51843 Alpha virt. eigenvalues -- 0.520420 .525990 .527170 .543800 .54403 Alpha virt. eigenvalues -- 0.546710 .549370 .554860 .558050 .55910 Alpha virt. eigenvalues -- 0.566080 .571980 .573370 .574810 .57804 Alpha virt. eigenvalues -- 0.582260 .582320 .585290 .589940 .59242 Alpha virt. eigenvalues -- 0.594390 .599400 .600910 .611820 .61485 Alpha virt. eigenvalues -- 0.617190 .617540 .620390 .628310 .62894 Alpha virt. eigenvalues -- 0.632780 .633300 .637690 .642110 .64509 Alpha virt. eigenvalues -- 0.645690 .649780 .652550 .652670 .65532 
Alpha virt. eigenvalues -- 0.660840 .667830 .668160 .669070 .67058 Alpha virt. eigenvalues -- 0.671450 .673680 .676300 .676510 .67797 Alpha virt. eigenvalues -- 0.679000 .681700 .681770 .683010 .68354 Alpha virt. eigenvalues -- 0.685240 .686300 .689800 .691440 .69328 Alpha virt. eigenvalues -- 0.693760 .694380 .696680 .697830 .69857 Alpha virt. eigenvalues -- 0.700620 .702990 .703370 .706210 .70647 Alpha virt. eigenvalues -- 0.707060 .707920 .709910 .713090 .71433 Alpha virt. eigenvalues -- 0.715480 .716280 .720390 .725000 .72548 Alpha virt. eigenvalues -- 0.728000 .728530 .728760 .730030 .73094 Alpha virt. eigenvalues -- 0.731060 .733420 .733520 .736090 .73846 Alpha virt. eigenvalues -- 0.738710 .740490 .741830 .745220 .75121 Alpha virt. eigenvalues -- 0.752230 .754110 .756630 .757650 .75797 Alpha virt. eigenvalues -- 0.760000 .760160 .764600 .765240 .76900 Alpha virt. eigenvalues -- 0.769870 .777220 .778840 .782180 .78273 Alpha virt. eigenvalues -- 0.784120 .791830 .793430 .796460 .79886 Alpha virt. eigenvalues -- 0.801450 .801980 .804420 .806580 .81211 Alpha virt. eigenvalues -- 0.813260 .814940 .819050 .821310 .82303 Alpha virt. eigenvalues -- 0.827360 .828780 .831300 .840150 .84074 Alpha virt. eigenvalues -- 0.841520 .844690 .846030 .846830 .84912 Alpha virt. eigenvalues -- 0.852750 .856130 .858010 .864520 .87640 Alpha virt. eigenvalues -- 0.878370 .880000 .887090 .900090 .90687 Alpha virt. eigenvalues -- 0.909150 .929650 .949000 .949110 .95864 Alpha virt. eigenvalues -- 0.959890 .962430 .967110 .991450 .99257 Alpha virt. eigenvalues -- 1.007831 .012461 .016521 .024571 .02835 Alpha virt. eigenvalues -- 1.031991 .032771 .041351 .054441 .05666 Alpha virt. eigenvalues -- 1.061481 .065051 .086731 .095111 .10170 Alpha virt. eigenvalues -- 1.102891 .106431 .119551 .120941 .12198 Alpha virt. eigenvalues -- 1.126501 .127301 .132451 .137371 .14325 Alpha virt. eigenvalues -- 1.146341 .154481 .160201 .167961 .17927 Alpha virt. eigenvalues -- 1.183031 .189671 .219451 .224801 .24155 Alpha virt. eigenvalues -- 1.243411 .251791 .259911 .262211 .28871 Alpha virt. eigenvalues -- 1.294881 .300441 .306131 .306471 .31410 Alpha virt. eigenvalues -- 1.328991 .329701 .351651 .356041 .35919 Alpha virt. eigenvalues -- 1.374881 .384821 .394851 .412691 .45279 Alpha virt. eigenvalues -- 1.457271 .481081 .508761 .538751 .59069 Alpha virt. eigenvalues -- 1.597471 .613271 .616701 .739741 .80011 Alpha virt. eigenvalues -- 1.826951 .89339

\section{Chloroform}

Alpha occ. eigenvalues -- -19.21501 -19.21501 -14.33888 -14.33887 -10.29169 Alpha occ. eigenvalues -- -10.29169 -10.25880 -10.25869-10.23389-10.23380 Alpha occ. eigenvalues -- -10.21847 -10.21836 -10.20720 -10.20720 -10.20043 Alpha occ. eigenvalues -- -10.20043 -10.19903 -10.19903-10.19784-10.19772 Alpha occ. eigenvalues -- -10.19576 -10.19576 -10.19228 -10.19228 -10.19130 Alpha occ. eigenvalues -- -10.19130 -10.18839-10.18839-10.18535-10.18535 Alpha occ. eigenvalues -- -10.18445 -10.18445 -10.17901 -10.17901 -10.17712 Alpha occ. eigenvalues -- -10.17711 -10.17550 -10.17550 -10.17549-10.17549 Alpha occ. eigenvalues -- -10.16142 -10.16142 -10.16140 -10.16140 -10.15987 Alpha occ. eigenvalues -- -10.15987 -10.15811 -10.15811 -10.15809-10.15809 Alpha occ. eigenvalues -- -10.15696 -10.15696 -1.14664 -1.14558 -0.95387 Alpha occ. eigenvalues -- $-0.95382-0.89726-0.88212-0.87789-0.87172$ Alpha occ. eigenvalues -- $-0.86381-0.83431-0.82961-0.82947-0.81871$ Alpha occ. eigenvalues -- $-0.79617-0.79127-0.77205-0.76073-0.76038$ Alpha occ. eigenvalues -- $-0.74987-0.74975-0.74944-0.73673-0.72316$ Alpha occ. eigenvalues -- -0.68969-0.68784 -0.68757 -0.68528 -0.68528 
Alpha occ. eigenvalues -- -0.68400 -0.68373 -0.68184-0.68184-0.66183 Alpha occ. eigenvalues -- $-0.65055-0.64627-0.62934-0.61627-0.61500$ Alpha occ. eigenvalues -- -0.61480 -0.61186 -0.59952 -0.59144-0.59058 Alpha occ. eigenvalues -- $-0.56739-0.56183-0.56063-0.55739-0.55385$ Alpha occ. eigenvalues -- $-0.55332-0.53315-0.52314-0.51539-0.50622$ Alpha occ. eigenvalues -- $-0.49960-0.49416-0.49169-0.47982-0.47854$ Alpha occ. eigenvalues -- $-0.47691-0.47455-0.47435-0.46995-0.46281$ Alpha occ. eigenvalues -- $-0.45763-0.45555-0.45084-0.45034-0.44794$ Alpha occ. eigenvalues -- $-0.44788-0.44657-0.44419-0.44414-0.44050$ Alpha occ. eigenvalues -- $-0.44009-0.43042-0.43037-0.42953-0.42879$ Alpha occ. eigenvalues -- $-0.42756-0.42405-0.41620-0.41201-0.40824$ Alpha occ. eigenvalues -- $-0.40473-0.40473-0.40193-0.40026-0.40008$ Alpha occ. eigenvalues -- $-0.39490-0.39372-0.38776-0.38225-0.38208$ Alpha occ. eigenvalues -- -0.38131 -0.38025 -0.37839-0.37791 -0.37318 Alpha occ. eigenvalues -- $-0.36995-0.36995-0.36711-0.36702-0.36675$ Alpha occ. eigenvalues -- $-0.36524-0.36119-0.35893-0.35574-0.35546$ Alpha occ. eigenvalues -- $-0.34680-0.34128-0.33745-0.33713-0.33710$ Alpha occ. eigenvalues -- $-0.33621-0.33294-0.33205-0.33092-0.32755$ Alpha occ. eigenvalues -- -0.32740 -0.32207 -0.32157 -0.30263 -0.29733 Alpha occ. eigenvalues -- $-0.29485-0.28770-0.27439-0.27380-0.26876$ Alpha occ. eigenvalues -- $-0.26341-0.26272-0.25228-0.23611-0.21797$ Alpha virt. eigenvalues -- $-0.09102-0.05756-0.04907-0.02270-0.02196$ Alpha virt. eigenvalues -- $-0.01448-0.01344-0.010610 .002890 .02535$ Alpha virt. eigenvalues -- 0.037670 .052700 .054140 .055730 .05811 Alpha virt. eigenvalues -- 0.058530 .069850 .077390 .086140 .08802 Alpha virt. eigenvalues -- 0.092280 .095070 .097820 .098280 .10354 Alpha virt. eigenvalues -- 0.103750 .104630 .105290 .110180 .11149 Alpha virt. eigenvalues -- 0.115430 .115970 .116160 .118060 .12033 Alpha virt. eigenvalues -- 0.120550 .121610 .125260 .125470 .12593 Alpha virt. eigenvalues -- 0.126490 .128120 .130460 .139380 .14271 Alpha virt. eigenvalues -- 0.142740 .144470 .144520 .147830 .15138 Alpha virt. eigenvalues -- 0.153430 .166270 .167330 .168070 .17070 Alpha virt. eigenvalues -- 0.173660 .173740 .174680 .175710 .17600 Alpha virt. eigenvalues -- 0.180780 .181430 .183200 .184730 .18535 Alpha virt. eigenvalues -- 0.189120 .189160 .190210 .191280 .19225 Alpha virt. eigenvalues -- 0.193310 .194620 .197400 .199640 .20058 Alpha virt. eigenvalues -- 0.203600 .205260 .205550 .218680 .22076 Alpha virt. eigenvalues -- 0.222490 .227950 .229260 .235280 .23884 Alpha virt. eigenvalues -- 0.240760 .241910 .244720 .244800 .24567 Alpha virt. eigenvalues -- 0.245890 .250080 .250110 .262210 .27056 Alpha virt. eigenvalues -- 0.275040 .278730 .279900 .281700 .28308 Alpha virt. eigenvalues -- 0.288100 .291440 .298370 .299390 .30545 Alpha virt. eigenvalues -- 0.312540 .315550 .319060 .320120 .32293 Alpha virt. eigenvalues -- 0.330440 .331260 .341670 .342270 .34252 Alpha virt. eigenvalues -- 0.351750 .352570 .360640 .364530 .37740 Alpha virt. eigenvalues -- 0.380720 .384950 .388360 .402010 .40809 Alpha virt. eigenvalues -- 0.414850 .417630 .418480 .418500 .42085 Alpha virt. eigenvalues -- 0.421540 .422100 .422350 .427530 .43048 Alpha virt. eigenvalues -- 0.431590 .436150 .436360 .442790 .44399 Alpha virt. eigenvalues -- 0.448000 .451230 .451320 .453920 .45516 Alpha virt. eigenvalues -- 0.457140 .462400 .464020 .465320 .46976 Alpha virt. eigenvalues -- 0.472540 .474960 .477200 .478010 .48196 Alpha virt. eigenvalues -- 0.483910 .484690 .491450 .491580 .49717 Alpha virt. eigenvalues -- 0.498900 .500380 .504800 .510970 .51099 Alpha virt. eigenvalues -- 0.514600 .518130 .521850 .537030 .53876 Alpha virt. eigenvalues -- 0.542070 .544630 .545630 .551280 .55457 
Alpha virt. eigenvalues -- 0.559410 .564840 .572190 .573950 .57399 Alpha virt. eigenvalues -- 0.577420 .578920 .581460 .585420 .58684 Alpha virt. eigenvalues -- 0.589890 .594400 .596270 .606810 .60732 Alpha virt. eigenvalues -- 0.611210 .618270 .621160 .624120 .62637 Alpha virt. eigenvalues -- 0.626580 .629180 .635360 .636150 .63899 Alpha virt. eigenvalues -- 0.646430 .646570 .647280 .649040 .65173 Alpha virt. eigenvalues -- 0.654090 .665170 .667320 .668870 .67105 Alpha virt. eigenvalues -- 0.671250 .671670 .673610 .674050 .67593 Alpha virt. eigenvalues -- 0.676120 .679320 .680580 .681280 .68232 Alpha virt. eigenvalues -- 0.683090 .683670 .686990 .690070 .69122 Alpha virt. eigenvalues -- 0.691540 .693210 .694040 .695740 .69581 Alpha virt. eigenvalues -- 0.697920 .699620 .700620 .701850 .70255 Alpha virt. eigenvalues -- 0.705130 .706760 .707730 .711060 .71132 Alpha virt. eigenvalues -- 0.713140 .713980 .718430 .721390 .72251 Alpha virt. eigenvalues -- 0.724760 .725350 .727180 .728050 .72932 Alpha virt. eigenvalues -- 0.730080 .731130 .734050 .735430 .73558 Alpha virt. eigenvalues -- 0.736680 .738890 .740610 .742690 .74762 Alpha virt. eigenvalues -- 0.748660 .749910 .755090 .755550 .75588 Alpha virt. eigenvalues -- 0.758510 .759600 .759760 .761870 .76224 Alpha virt. eigenvalues -- 0.765500 .770320 .772860 .776200 .77831 Alpha virt. eigenvalues -- 0.779380 .786910 .790700 .791430 .79353 Alpha virt. eigenvalues -- 0.796680 .796810 .800420 .801960 .80712 Alpha virt. eigenvalues -- 0.810050 .810100 .815030 .815260 .81578 Alpha virt. eigenvalues -- 0.821440 .822150 .825660 .835630 .83862 Alpha virt. eigenvalues -- 0.838780 .844670 .845210 .845740 .84724 Alpha virt. eigenvalues -- 0.847660 .848070 .852850 .858480 .86764 Alpha virt. eigenvalues -- 0.871850 .872960 .879490 .894940 .90154 Alpha virt. eigenvalues -- 0.902900 .919590 .937950 .938720 .94932 Alpha virt. eigenvalues -- 0.951770 .954720 .962380 .984330 .98512 Alpha virt. eigenvalues -- 1.000051 .001811 .011021 .019061 .02040 Alpha virt. eigenvalues -- 1.026081 .028121 .034341 .049691 .05110 Alpha virt. eigenvalues -- 1.053251 .063161 .081701 .085491 .09661 Alpha virt. eigenvalues -- 1.100401 .101031 .112581 .116491 .11935 Alpha virt. eigenvalues -- 1.122271 .125541 .125971 .127241 .12954 Alpha virt. eigenvalues -- 1.136411 .149701 .150431 .153881 .16727 Alpha virt. eigenvalues -- 1.175871 .181581 .186671 .208171 .21871 Alpha virt. eigenvalues -- 1.231941 .234301 .253471 .256401 .28550 Alpha virt. eigenvalues -- 1.288351 .292081 .293871 .300551 .30752 Alpha virt. eigenvalues -- 1.320301 .320591 .344051 .349311 .35171 Alpha virt. eigenvalues -- 1.361571 .377391 .384881 .409991 .44520 Alpha virt. eigenvalues -- 1.446741 .473321 .499711 .532691 .58587 Alpha virt. eigenvalues -- 1.593251 .602111 .611561 .727041 .79380 Alpha virt. eigenvalues -- 1.817651 .88152

\section{Comparison of Experimental and DFT UV-Vis}

Gas Phase Chloroform



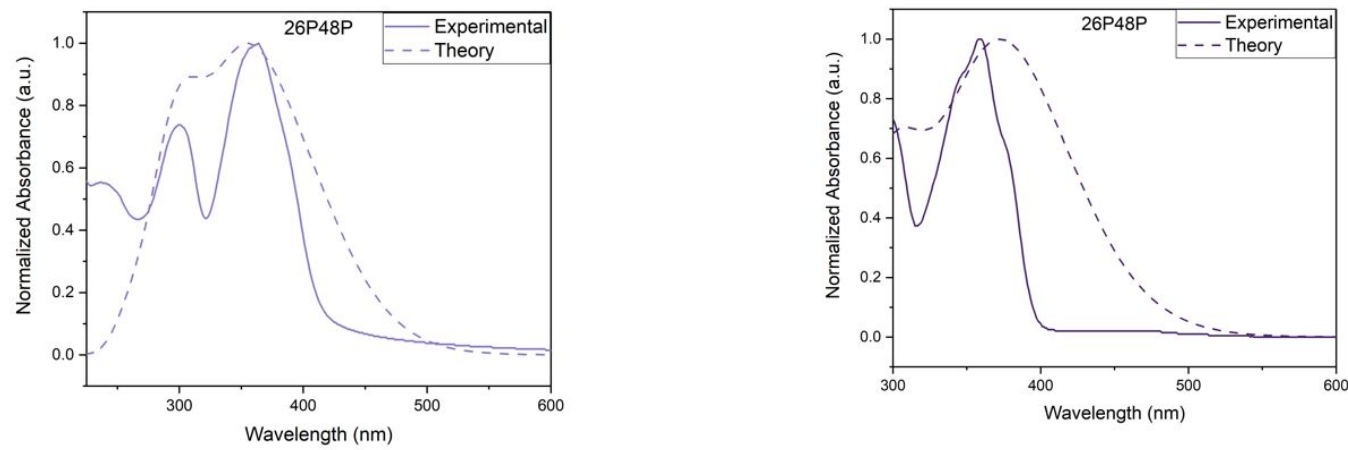

Figure S32. Gas phase and chloroform overlays of computation and experimental data for $26 \mathrm{P} 48 \mathrm{P}$.

\section{Lowest 15 Excited States}

\begin{tabular}{|c|c|c|c|c|c|c|}
\hline \multicolumn{4}{|c|}{ Gas Phase } & \multicolumn{3}{|c|}{ Chloroform } \\
\hline $\begin{array}{l}\text { Excited } \\
\text { state }\end{array}$ & $\begin{array}{l}\text { Energy } \\
(\mathrm{eV})\end{array}$ & $\frac{\text { Wavelength }}{(\mathrm{nm})}$ & $f$ & $\frac{\text { Energy }}{(\mathrm{eV})}$ & $\frac{\text { Wavelength }}{(\mathrm{nm})}$ & $f$ \\
\hline 1 & 3.08 & $\longdiv { 4 0 2 . 4 2 }$ & 0.59 & 3.09 & 401.22 & 0.89 \\
\hline 2 & 3.52 & 352.46 & 1.09 & 3.45 & 359.67 & 1.17 \\
\hline 3 & 3.78 & 328.11 & 0.00 & 3.87 & 320.76 & 0.00 \\
\hline 4 & 3.90 & 317.57 & 0.00 & 3.89 & 318.37 & 0.00 \\
\hline 5 & 4.07 & 304.78 & 0.04 & 4.09 & 303.50 & 1.03 \\
\hline 6 & 4.09 & 303.38 & 0.00 & 4.14 & 299.61 & 0.01 \\
\hline 7 & 4.12 & 301.07 & 1.00 & 4.16 & 297.97 & 0.00 \\
\hline 8 & 4.38 & 282.75 & 0.00 & 4.37 & 283.79 & 0.00 \\
\hline 9 & 4.50 & 275.58 & 0.01 & 4.44 & 279.15 & 0.02 \\
\hline 10 & 4.51 & 274.65 & 0.00 & 4.46 & 278.18 & 0.00 \\
\hline 11 & 4.58 & 270.45 & 0.00 & 4.51 & 274.98 & 0.00 \\
\hline 12 & 4.64 & 267.49 & 0.22 & 4.62 & 268.49 & 0.00 \\
\hline 13 & 4.67 & 265.74 & 0.00 & 4.76 & 260.63 & 0.00 \\
\hline 14 & 4.75 & 261.05 & 0.00 & 4.79 & 258.64 & 0.00 \\
\hline 15 & 4.76 & 260.62 & 0.03 & 4.84 & 256.30 & 0.18 \\
\hline
\end{tabular}

Table S8. Gas phase and chloroform excitation energies, wavelengths, and oscillator strengths for first 15 excited state of $26 \mathrm{P} 48 \mathrm{P}$.

\section{Geometric Images}

Gas Phase
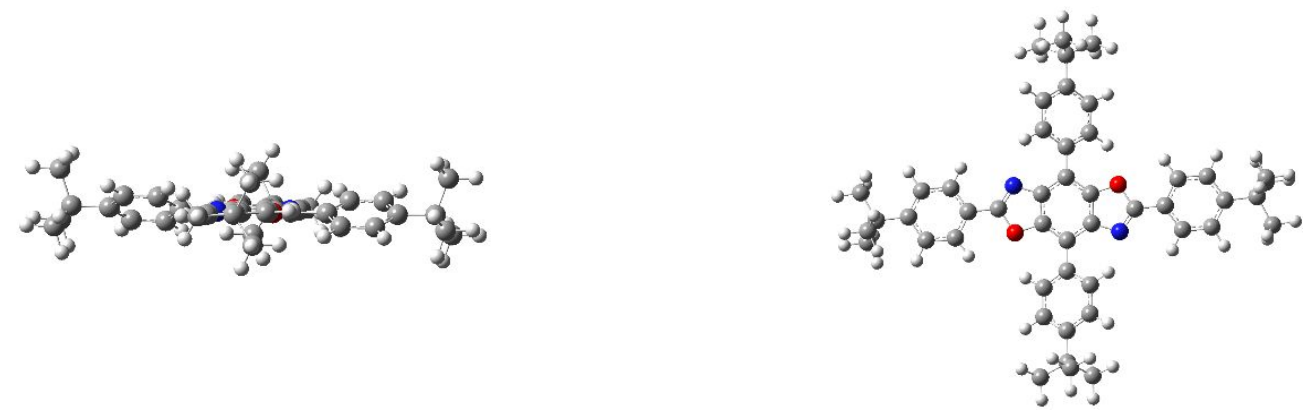


\section{Chloroform}
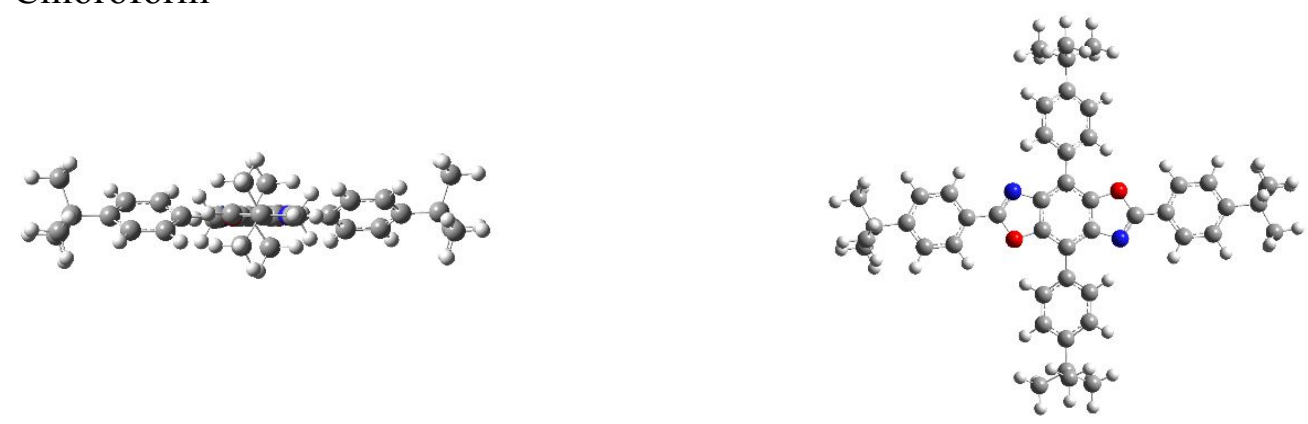

Figure S33. Geometric optimization images in gas phase and chloroform for 26P48P.

\section{P48M}

\section{Cartesian Coordinates}

\section{Gas Phase}

C $-1.50064-0.13431-0.0829$

C $-0.79971 .0773-0.083$

C $1.500610 .1343-0.08321$

C $0.79968-1.07731-0.08322$

C $0.612871 .23719-0.09385$

C $-0.247983 .23502-0.10747$

O $-1.348022 .36114-0.09118$

N $0.90312 .60743-0.10713$

C $-0.6129-1.2372-0.09378$

C $0.24795-3.23503-0.10769$

$\mathrm{N}-0.90313-2.60744-0.10707$

O $1.348-2.36115-0.09157$

C $2.97340 .25022-0.0592$

C $3.61741 .32357-0.70544$

C $3.7559-0.704170 .62188$

C $5.018411 .45392-0.67985$

H $3.014652 .05439-1.24607$

C $5.15816-0.594270 .67235$

H $3.26209-1.520071 .1532$

C $5.684362 .5881-1.37173$

C 5.772790 .48830 .01418

C $5.96951-1.589811 .42056$

C $5.079383 .85764-1.43973$

C $6.946642 .43889-1.9845$

H 6.862050 .582460 .04615

C $7.12902-1.209332 .12925$

C $5.60918-2.950081 .45722$

C $5.707744 .93066-2.08687$

H $4.108634 .01798-0.95921$

C $7.569693 .51203-2.62814$

H $7.438671 .46114-1.9807$

C $7.88503-2.148462 .83677$

H $7.42901-0.156892 .15109$

C $6.37033-3.888922 .16747$

H $4.72743-3.288940 .90387$

C $6.968524 .78715-2.69638$

H $5.195225 .89503-2.1022$ 
H $8.54553 .34307-3.09478$

C $7.52731-3.513322 .8757$

H $8.77005-1.8013 .3795$

H $6.04575-4.931852 .15474$

C - $2.97342-0.25022-0.05859$

C $-3.61755-1.32355-0.70474$

C -3.755780 .704140 .62267$

C $-5.01856-1.4539-0.67886$

H -3.01492 -2.05436 -1.24551

C -5.158030 .594240 .67343$

$\mathrm{H}-3.261851 .520011 .15393$

C -5.68465 -2.58806 - 1.37064

C $-5.7728-0.48830 .01534$

C -5.969221 .589771 .42184$

C $-5.07969-3.8576-1.43877$

C $-6.94704-2.43882-1.98319$

H -6.86205 -0.58246 0.04753

C -7.12861 1.20929 2.13071

C -5.608842 .950031 .4585$

C $-5.70818-4.93061-2.08582$

H $-4.10887-4.01797-0.95842$

C -7.5702 -3.51194 -2.62674

H -7.43905 -1.46107 -1.97929

C -7.884472 .148412 .83841$

$\mathrm{H}-7.428630 .156862 .15255$

C -6.36984 3.88885 2.16894

H -4.727183 .288890 .90501$

C -6.96907 -4.78707 -2.69511

H -5.19567 -5.89498 -2.10126

H -8.54609 -3.34297 -3.09322

C -7.52673 .513252 .87735$

$\mathrm{H}-8.769411 .800943 .38127$

H -6.04523 4.931782 .1562

C $8.38486-4.511313 .67386$

C $8.38941-4.100875 .16817$

C $7.84634-5.952133 .56957$

C $9.83592-4.495893 .13147$

H 8.80371 -3.08605 5.31192

H 7.36297 -4.109 5.58054

H $9.00606-4.806095 .75869$

H $7.8401-6.31092 .52329$

H $8.49223-6.634534 .15347$

H $6.82037-6.036473 .97373$

H $10.46435-5.211323 .69667$

H $9.85936-4.783472 .06353$

H $10.29764-3.495663 .22326$

C $7.690775 .94208-3.41283$

C $7.935785 .55729-4.89358$

C $6.870217 .24709-3.38236$

C $9.050386 .20704-2.71843$

H $8.55964 .64898-4.98265$

H $6.978355 .36303-5.41242$

H $8.456596 .37883-5.42285$

H $6.686077 .59037-2.34725$

H $7.424618 .04814-3.90643$

H 5.894427 .1287 -3.88945 
H $9.584797 .03527-3.22322$

H $8.90196 .48603-1.65832$

H 9.70444 5.31616-2.74572

C -8.384094 .511233 .67571$

C -8.38844 4.100695.17

C -7.8455 5.95202 3.57145

C -9.835224 .495923 .13353$

$\mathrm{H}-8.802783 .085885 .31373$

H -7.36194 4.10872 5.58221

H -9.004964 .80595 .76065$

$\mathrm{H}-7.839396 .310872 .52519$

H -8.49127 6.63442 4.15549

H -6.81947 6.03629 3.97547

H - 10.463545 .211343 .69887

H -9.85884 .783582 .06562$

$\mathrm{H}-10.296983 .495713 .22531$

C - -7.69144 -5.94198 -3.41147

C $-7.93666-5.55716-4.89218$

C $-6.8709-7.247-3.38114$

C $-9.05096-6.20692-2.71688$

$\mathrm{H}-8.56047-4.64881-4.98114$

H -6.97931 -5.36292 -5.41117

$\mathrm{H}-8.45758-6.37866-5.42139$

H -6.68659 -7.59029 -2.34607

H -7.4254 -8.04804 -3.90513

H $-5.89519-7.12863-3.88839$

H $-9.58544-7.03514-3.2216$

H -8.90233 -6.48593 -1.65679

H $-9.70501-5.31604-2.74407$

C $0.50792-4.66148-0.11858$

C $-0.56653-5.57081-0.02723$

C $1.81598-5.16784-0.22151$

C $-0.32674-6.9444-0.03603$

$\mathrm{H}-1.58448-5.179790 .05581$

C $2.04372-6.54921-0.23011$

H $2.65616-4.47267-0.30128$

C $0.98213-7.47034-0.13672$

H - $1.18137-7.623260 .04036$

H $3.07343-6.90217-0.313$

C $-0.507954 .66147-0.11823$

C - $1.816035 .16783-0.22092$

C $0.566515 .5708-0.02701$

C -2.04378 $6.5492-0.2294$

H -2.65622 4.47266 -0.30059

C $0.326716 .9444-0.03569$

H 1.584485 .179780 .05584

C $-0.982187 .47034-0.13614$

$\mathrm{H}-3.073516 .90216-0.3121$

H 1.181357 .623250 .04061

C - $1.196318 .99357-0.1369$

C -2.6861 9.37166 -0.25675

C -0.648169 .587051 .18584$

C $-0.437839 .61824-1.33536$

H -3.12974 8.99736-1.19815

H -3.27829 8.97785 0.5902

H -2.79116 10.47268 -0.25345 
H 0.431379 .384651 .31049

H -0.7904 10.684781 .19906

H -1.17703 9.15953 2.05824

H - $0.5831310 .71568-1.34775$

H $0.648689 .42232-1.28112$

H -0.80987 9.20948 -2.2935

C $1.19625-8.99358-0.13762$

C $2.68601-9.37167-0.25776$

C $0.64834-9.587141 .18519$

C $0.43754-9.61817-1.33598$

H $3.12948-8.99733-1.19923$

H $3.27837-8.97790 .58909$

H $2.79107-10.47269-0.25454$

H - $0.43116-9.384751 .31005$

H $0.79059-10.684881 .19831$

H $1.17737-9.159682 .05751$

H $0.58283-10.71561-1.34846$

H - $0.64895-9.42224-1.28153$

H $0.80942-9.20935-2.29416$

\section{Chloroform}

C $0.79798-1.07906-0.077$

C $-0.797971 .07907-0.077$

C $0.614121 .23697-0.08542$

N $0.9062 .60685-0.09145$

C $-0.244963 .23748-0.09325$

O - $1.34492 .36286-0.08479$

C $0.24496-3.23748-0.09329$

C $-0.61412-1.23697-0.08545$

O $1.3449-2.36285-0.0848$

$\mathrm{N}-0.906-2.60685-0.09151$

C - $1.49903-0.13209-0.07786$

C $1.499030 .13209-0.07782$

C $-2.9727-0.2466-0.05741$

C $-3.61721-1.30032-0.73578$

C -3.75430 .689360 .6505$

C -5.01905-1.43084-0.71425

H -3.01445 -2.01337-1.30023

C -5.157580 .579010 .69705$

H -3.2591 .489941 .20394$

C $-5.7729-0.48470 .00796$

C -5.68759-2.54292 -1.44002

C -5.970441 .55341 .4722$

H $-6.86209-0.579130 .03687$

C $-5.08349-3.81063-1.55074$

C $-6.95313-2.37332-2.04268$

C -5.606842 .911681 .55238$

C -7.135821 .153072 .16163$

C $-5.71558-4.86306-2.22891$

H $-4.11154-3.99123-1.08018$

C - $7.57925-3.42553-2.71864$

H -7.44822 -1.39785 -2.00431

C -6.371623 .830582 .28592$

H -4.721853 .268391 .01592$

C -7.894342 .072142 .89378$ 
H -7.44196 0.10229 2.14792

C $-6.9789-4.69906-2.82957$

H -5.20369 $-5.82678-2.27481$

H -8.55691 -3.24029 -3.17491

C -7.534133 .435352 .97575$

H -6.04543 4.87284 2.30541

H -8.7835 1.709633 .41934

C $-7.7031-5.83013-3.58071$

C -8.394054 .410793 .7986$

C $-9.06788-6.10692-2.90093$

C $-6.88845-7.13912-3.58259$

C -7.93946 -5.40111 -5.05093

C -8.394113 .963195 .28247$

C -7.860945 .855583 .72752$

C -9.846534 .401763 .25948$

H $-9.71818-5.21282-2.90756$

H $-9.60183-6.91774-3.43317$

H -8.92672 -6.41929-1.84892

H -6.70905 -7.51116 -2.55662

H -7.44592 -7.92201 -4.12999

H -5.91049 -7.01232 -4.08337

H $-8.55723-4.48654-5.11627$

H -6.97835 -5.20177 -5.56153

H -8.46402 -6.20441 -5.60351

H -9.015584 .650225 .88878$

H -8.8021 2.94249 5.40128

$\mathrm{H}-7.367513 .971165 .69512$

H -7.85548 6.23857 2.68983

H - 8.510376 .52054 .32718

H -6.835515 .93514 .13391$

H - 10.473585 .103513 .84262

H -9.874324 .714462 .19852$

H - 10.305953 .398863 .33259

C $2.97270 .24661-0.05733$

C $3.617231 .30033-0.73566$

C $3.75428-0.689370 .6506$

C $5.019081 .43085-0.71408$

H $3.014492 .01338-1.30013$

C $5.15756-0.579020 .6972$

H $3.25895-1.489951 .20403$

C 5.77290 .48470 .00815

C $5.687642 .54294-1.4398$

C $5.97039-1.553421 .47237$

H 6.862090 .579120 .03709

C $5.083543 .81065-1.55053$

C $6.95322 .37334-2.04243$

C $5.60676-2.91171 .55254$

C $7.13576-1.153112 .16182$

C $5.715664 .86309-2.22866$

H $4.111573 .99124-1.08$

C $7.579343 .42557-2.71834$

H $7.448291 .39788-2.00405$

C $6.37151-3.830612 .28609$

H 4.72178 -3.26839 1.01606

C $7.89424-2.07222 .89398$

H $7.44191-0.102342 .14812$ 
C $6.9794 .6991-2.82928$

H $5.203775 .82681-2.27456$

H $8.557023 .24033-3.17458$

C $7.53401-3.43542 .97595$

H $6.0453-4.872872 .30558$

H $8.78341-1.70973 .41956$

C $7.703235 .83018-3.58037$

C 8.3939 -4.41086 3.79881

C $9.067976 .10699-2.90051$

C $6.888557 .13916-3.58229$

C $7.939675 .40117-5.05058$

C 8.39403 -3.96319 5.28266

C $7.8607-5.855623 .72781$

C $9.84637-4.401933 .25965$

H $9.718285 .2129-2.90711$

H $9.601936 .91783-3.43272$

H $8.926746 .41935-1.84851$

H $6.709087 .51118-2.55633$

H $7.446067 .92207-4.12964$

H $5.910637 .01235-4.08314$

H $8.557464 .4866-5.11589$

H $6.97865 .20182-5.56123$

H $8.464266 .20447-5.60312$

H $9.01548-4.650235 .88898$

H $8.80208-2.942515 .40141$

H $7.36744-3.971095 .69535$

H $7.85516-6.238642 .69013$

H $8.51012-6.520564 .32746$

H $6.83529-5.935074 .13426$

H $10.47339-5.10373 .84279$

H 9.8741 -4.71468 2.1987

H $10.30585-3.399063 .3327$

C $-0.504254 .66381-0.09853$

C $0.571745 .57456-0.02617$

C - $1.81515 .16996-0.17635$

C $0.331066 .94841-0.02896$

H 1.593615 .19010 .03616

C -2.04394 $6.5514-0.17897$

H -2.65871 4.47757 -0.23917

C $-0.980667 .47342-0.10454$

H 1.18717 .626790 .03147

H -3.07548 $6.90284-0.24087$

C $0.50426-4.66381-0.09859$

C $1.81511-5.16995-0.17635$

C $-0.57173-5.57456-0.0263$

C $2.04396-6.55138-0.17899$

H $2.65872-4.47755-0.23913$

C -0.33105 -6.94841-0.0291

$\mathrm{H}-1.59361-5.190110 .03599$

C $0.98068-7.47341-0.10462$

H $3.07551-6.90282-0.24085$

H - 1.18709 -7.6268 0.03129

C - $1.196048 .99593-0.09908$

C $1.19606-8.99592-0.09918$

C $-0.453519 .6233-1.30638$

H $0.633989 .42912-1.2665$ 
H $-0.839899 .21804-2.26053$

H -0.60099 $10.72029-1.31239$

C -0.628299 .585611 .2174$

$\mathrm{H}-0.7709210 .683071 .23314$

H - 1.146759 .157722 .09607

H 0.453159 .383381 .32522

C -2.68758 9.37301 -0.19698

H -3.14445 $8.99988-1.13252$

H -3.267698.97771 0.65758

H -2.7914 $10.47401-0.19039$

C $0.62831-9.58561 .2173$

H - $0.45312-9.383341 .32513$

H $0.77091-10.683061 .23303$

H $1.14678-9.157732 .09598$

C $2.68761-9.37299-0.19706$

H $3.14449-8.99984-1.13259$

H $3.26771-8.977710 .65751$

H $2.79143-10.47399-0.19049$

C $0.45355-9.6233-1.30648$

H - $0.63395-9.42916-1.26659$

H $0.83991-9.21801-2.26063$

H $0.60107-10.72028-1.3125$

\section{Energy Levels}

\section{Gas Phase}

Alpha occ. eigenvalues -- -19.20882 -19.20881 -14.33261 -14.33260 -10.28523

Alpha occ. eigenvalues -- -10.28523 -10.25274 -10.25263 -10.22757-10.22748

Alpha occ. eigenvalues -- $-10.21202-10.21192-10.20374-10.20374-10.20368$

Alpha occ. eigenvalues -- -10.20368 -10.19796 -10.19796 -10.19492 -10.19492

Alpha occ. eigenvalues -- -10.19389 -10.19389-10.19332 -10.19319-10.19039

Alpha occ. eigenvalues -- -10.19038 -10.18910 -10.18910 -10.18577 -10.18577

Alpha occ. eigenvalues -- -10.18483 -10.18483 -10.18455 -10.18455 -10.18410

Alpha occ. eigenvalues -- -10.18410 -10.18382 -10.18381 -10.18233 -10.18233

Alpha occ. eigenvalues -- -10.18205 -10.18205 -10.18090 -10.18090 -10.17351

Alpha occ. eigenvalues -- -10.17350 -10.17345 -10.17345 -10.17341 -10.17341

Alpha occ. eigenvalues -- -10.17306 -10.17306 -10.17218 -10.17218 -10.17211

Alpha occ. eigenvalues -- -10.17211 -10.17161 -10.17161 -10.17084-10.17084

Alpha occ. eigenvalues -- -10.17072 -10.17072 -10.17027 -10.17026 -10.16971

Alpha occ. eigenvalues -- -10.16971 -10.16564 -10.16564-10.16556-10.16556

Alpha occ. eigenvalues -- -10.16527 -10.16527 -10.15863 -10.15863 -10.15844

Alpha occ. eigenvalues -- -10.15844 -10.15807 -10.15807-10.15760 -10.15760

Alpha occ. eigenvalues -- -10.15754 -10.15754 -10.15721 -10.15721 -1.14013

Alpha occ. eigenvalues -- $-1.13907-0.94815-0.94810-0.89294-0.88047$

Alpha occ. eigenvalues -- $-0.87786-0.87711-0.87034-0.86479-0.86474$

Alpha occ. eigenvalues -- $-0.85606-0.85185-0.83489-0.83023-0.82235$

Alpha occ. eigenvalues -- $-0.82227-0.82032-0.81477-0.80128-0.79106$

Alpha occ. eigenvalues -- $-0.77515-0.77440-0.77434-0.76382-0.75929$

Alpha occ. eigenvalues -- $-0.75925-0.74621-0.74483-0.74390-0.74319$

Alpha occ. eigenvalues -- $-0.74197-0.73417-0.72507-0.71564-0.71222$

Alpha occ. eigenvalues -- $-0.71145-0.69256-0.69188-0.68930-0.68930$

Alpha occ. eigenvalues -- -0.68585 -0.68424 -0.68417-0.68320 -0.68317

Alpha occ. eigenvalues -- $-0.68192-0.68192-0.68101-0.68101-0.65833$

Alpha occ. eigenvalues -- $-0.64873-0.64452-0.63593-0.63229-0.63229$

Alpha occ. eigenvalues -- -0.62416 -0.62405 -0.61081 -0.60824-0.60780

Alpha occ. eigenvalues -- $-0.60373-0.60352-0.59964-0.59229-0.58564$ 
Alpha occ. eigenvalues -- -0.57938 -0.57677 -0.56988 -0.56188 -0.56160 Alpha occ. eigenvalues -- $-0.56147-0.55446-0.55188-0.55183-0.55033$ Alpha occ. eigenvalues -- $-0.55030-0.53579-0.53220-0.52735-0.50186$ Alpha occ. eigenvalues -- $-0.50042-0.49717-0.49704-0.49369-0.49238$ Alpha occ. eigenvalues -- $-0.48962-0.48490-0.48344-0.48316-0.48067$ Alpha occ. eigenvalues -- $-0.47827-0.47796-0.47207-0.46752-0.46747$ Alpha occ. eigenvalues -- $-0.46570-0.46039-0.45832-0.45829-0.45210$ Alpha occ. eigenvalues -- $-0.45203-0.45178-0.45150-0.45056-0.44762$ Alpha occ. eigenvalues -- $-0.44701-0.44628-0.44489-0.44450-0.44428$ Alpha occ. eigenvalues -- $-0.44355-0.44326-0.44228-0.44219-0.44073$ Alpha occ. eigenvalues -- $-0.43961-0.43937-0.43219-0.43120-0.42804$ Alpha occ. eigenvalues -- -0.42764 -0.42624 -0.42591 -0.42168 -0.42157 Alpha occ. eigenvalues -- $-0.42003-0.42002-0.40944-0.40828-0.40669$ Alpha occ. eigenvalues -- -0.40560 -0.40181 -0.39948 -0.39860 -0.39794 Alpha occ. eigenvalues -- $-0.39745-0.39653-0.39395-0.39351-0.39322$ Alpha occ. eigenvalues -- $-0.39109-0.38931-0.38851-0.38677-0.38676$ Alpha occ. eigenvalues -- $-0.38421-0.38242-0.37982-0.37970-0.37857$ Alpha occ. eigenvalues -- $-0.37826-0.37449-0.37436-0.37010-0.36986$ Alpha occ. eigenvalues -- $-0.36753-0.36752-0.36745-0.36711-0.36643$ Alpha occ. eigenvalues -- $-0.36615-0.36592-0.36472-0.36357-0.36051$ Alpha occ. eigenvalues -- $-0.35778-0.35765-0.35229-0.35195-0.34749$ Alpha occ. eigenvalues -- $-0.34691-0.34651-0.34583-0.34389-0.34336$ Alpha occ. eigenvalues -- $-0.33959-0.33948-0.33621-0.33607-0.33414$ Alpha occ. eigenvalues -- $-0.33366-0.33117-0.33056-0.33040-0.32867$ Alpha occ. eigenvalues -- $-0.32864-0.32543-0.32523-0.32071-0.32061$ Alpha occ. eigenvalues -- -0.31917 -0.31911 -0.29679 -0.29329-0.29176 Alpha occ. eigenvalues -- $-0.28801-0.28339-0.28258-0.27422-0.27157$ Alpha occ. eigenvalues -- $-0.27157-0.25797-0.25793-0.25694-0.25625$ Alpha occ. eigenvalues -- $-0.25604-0.24452-0.23310-0.23045-0.22218$ Alpha occ. eigenvalues -- $-0.22189-0.21401$

Alpha virt. eigenvalues -- $-0.08551-0.05311-0.04558-0.03770-0.03743$ Alpha virt. eigenvalues -- $-0.02708-0.02024-0.01985-0.01352-0.00915$ Alpha virt. eigenvalues -- $-0.00887-0.00740-0.007130 .006310 .01248$ Alpha virt. eigenvalues -- 0.032910 .033220 .036810 .041510 .04654 Alpha virt. eigenvalues -- 0.046870 .055190 .055250 .056690 .05686 Alpha virt. eigenvalues -- 0.059590 .063520 .064900 .070180 .07039 Alpha virt. eigenvalues -- 0.085400 .085510 .089150 .089370 .09043 Alpha virt. eigenvalues -- 0.093080 .094420 .095760 .100080 .10235 Alpha virt. eigenvalues -- 0.102500 .103810 .103950 .105040 .10810 Alpha virt. eigenvalues -- 0.109110 .110330 .111140 .111510 .11222 Alpha virt. eigenvalues -- 0.114530 .115520 .116840 .117110 .11789 Alpha virt. eigenvalues -- 0.118110 .118420 .118750 .119950 .12113 Alpha virt. eigenvalues -- 0.121880 .123930 .126420 .126540 .12823 Alpha virt. eigenvalues -- 0.129530 .133350 .133700 .139090 .13979 Alpha virt. eigenvalues -- 0.142000 .142930 .143370 .144980 .14799 Alpha virt. eigenvalues -- 0.150930 .151420 .152630 .154080 .15527 Alpha virt. eigenvalues -- 0.158170 .160150 .162100 .162790 .16725 Alpha virt. eigenvalues -- 0.167310 .168620 .169050 .169170 .17097 Alpha virt. eigenvalues -- 0.172450 .173610 .174760 .175170 .17526 Alpha virt. eigenvalues -- 0.175780 .178360 .178880 .179370 .18111 Alpha virt. eigenvalues -- 0.182960 .184550 .184610 .185200 .19069 Alpha virt. eigenvalues -- 0.190800 .191590 .191930 .191990 .19281 Alpha virt. eigenvalues -- 0.193120 .194420 .196850 .197850 .19938 Alpha virt. eigenvalues -- 0.200550 .204220 .205660 .205990 .20818 Alpha virt. eigenvalues -- 0.209960 .210400 .214150 .217370 .21910 Alpha virt. eigenvalues -- 0.219920 .224810 .236600 .237720 .23886 
Alpha virt. eigenvalues -- 0.241210 .241320 .241660 .241670 .24250 Alpha virt. eigenvalues -- 0.244430 .244570 .249410 .249730 .25027 Alpha virt. eigenvalues -- 0.250290 .250820 .258250 .260740 .26621 Alpha virt. eigenvalues -- 0.267010 .270950 .272940 .273970 .27502 Alpha virt. eigenvalues -- 0.277100 .278720 .280880 .281130 .28621 Alpha virt. eigenvalues -- 0.287240 .288970 .289990 .293060 .29606 Alpha virt. eigenvalues -- 0.300540 .300800 .303300 .303490 .30678 Alpha virt. eigenvalues -- 0.310470 .311830 .316640 .316720 .31999 Alpha virt. eigenvalues -- 0.320730 .320870 .323760 .329010 .33022 Alpha virt. eigenvalues -- 0.332180 .335580 .336170 .340610 .34124 Alpha virt. eigenvalues -- 0.342440 .342630 .344870 .344880 .34903 Alpha virt. eigenvalues -- 0.362780 .363760 .364430 .365010 .36542 Alpha virt. eigenvalues -- 0.367810 .374700 .380280 .383460 .38511 Alpha virt. eigenvalues -- 0.387510 .393700 .394710 .397000 .39744 Alpha virt. eigenvalues -- 0.400660 .404070 .410650 .410910 .41261 Alpha virt. eigenvalues -- 0.412630 .414330 .414330 .418520 .42127 Alpha virt. eigenvalues -- 0.421280 .422150 .422150 .423190 .42835 Alpha virt. eigenvalues -- 0.430100 .433100 .437360 .437430 .44066 Alpha virt. eigenvalues -- 0.441100 .442020 .442110 .443320 .44501 Alpha virt. eigenvalues -- 0.446550 .447480 .447580 .449910 .45317 Alpha virt. eigenvalues -- 0.454160 .454170 .456810 .462610 .46280 Alpha virt. eigenvalues -- 0.464420 .466270 .466400 .468250 .46887 Alpha virt. eigenvalues -- 0.469960 .471390 .473490 .474080 .48065 Alpha virt. eigenvalues -- 0.480800 .482420 .482520 .483140 .48454 Alpha virt. eigenvalues -- 0.485400 .487740 .487830 .487980 .49300 Alpha virt. eigenvalues -- 0.495170 .496680 .497830 .501050 .50451 Alpha virt. eigenvalues -- 0.504960 .507880 .511110 .511350 .51235 Alpha virt. eigenvalues -- 0.515300 .516750 .519450 .519970 .52267 Alpha virt. eigenvalues -- 0.525620 .526270 .527930 .528310 .54093 Alpha virt. eigenvalues -- 0.541530 .546710 .548300 .552690 .55289 Alpha virt. eigenvalues -- 0.554510 .560890 .562700 .563350 .56498 Alpha virt. eigenvalues -- 0.569440 .571540 .572710 .573100 .57643 Alpha virt. eigenvalues -- 0.576890 .577330 .578810 .580540 .58332 Alpha virt. eigenvalues -- 0.585840 .586830 .589660 .592700 .59292 Alpha virt. eigenvalues -- 0.599460 .601080 .601770 .606230 .61074 Alpha virt. eigenvalues -- 0.611980 .612890 .615990 .616960 .61849 Alpha virt. eigenvalues -- 0.621470 .622090 .622480 .623910 .62392 Alpha virt. eigenvalues -- 0.626510 .628020 .628990 .630060 .63201 Alpha virt. eigenvalues -- 0.634570 .635260 .638110 .638790 .64008 Alpha virt. eigenvalues -- 0.641370 .645410 .645430 .647900 .65112 Alpha virt. eigenvalues -- 0.652420 .652480 .659530 .661220 .66244 Alpha virt. eigenvalues -- 0.663550 .664810 .665810 .666400 .66887 Alpha virt. eigenvalues -- 0.671570 .672290 .672550 .672760 .67364 Alpha virt. eigenvalues -- 0.674600 .674780 .675430 .676890 .67705 Alpha virt. eigenvalues -- 0.677800 .678050 .679360 .679890 .68171 Alpha virt. eigenvalues -- 0.682030 .684340 .685740 .687620 .68853 Alpha virt. eigenvalues -- 0.688980 .689160 .690270 .690960 .69311 Alpha virt. eigenvalues -- 0.693710 .694180 .695450 .695980 .69656 Alpha virt. eigenvalues -- 0.697620 .698020 .698110 .699530 .70060 Alpha virt. eigenvalues -- 0.701600 .703380 .703520 .704580 .70573 Alpha virt. eigenvalues -- 0.706230 .707290 .707820 .708580 .70970 Alpha virt. eigenvalues -- 0.710630 .712390 .712720 .713670 .71553 Alpha virt. eigenvalues -- 0.717200 .719710 .721840 .723110 .72380 Alpha virt. eigenvalues -- 0.725030 .725970 .726600 .726620 .72748 Alpha virt. eigenvalues -- 0.727760 .729170 .729250 .731300 .73174 Alpha virt. eigenvalues -- 0.732290 .732830 .733990 .734030 .73517 
Alpha virt. eigenvalues -- 0.737830 .737850 .739850 .740880 .74126 Alpha virt. eigenvalues -- 0.744910 .746010 .746380 .747790 .74795 Alpha virt. eigenvalues -- 0.751630 .752300 .752770 .754150 .75437 Alpha virt. eigenvalues -- 0.754940 .755020 .755930 .756850 .75852 Alpha virt. eigenvalues -- 0.759480 .760900 .762390 .763380 .76670 Alpha virt. eigenvalues -- 0.767080 .768070 .768270 .772260 .77233 Alpha virt. eigenvalues -- 0.777460 .778340 .778800 .782430 .78462 Alpha virt. eigenvalues -- 0.785340 .787070 .788570 .788620 .79269 Alpha virt. eigenvalues -- 0.793980 .794230 .796630 .799150 .80004 Alpha virt. eigenvalues -- 0.800940 .801670 .806300 .807530 .80763 Alpha virt. eigenvalues -- 0.810330 .811400 .816490 .818890 .82056 Alpha virt. eigenvalues -- 0.822030 .822310 .823400 .825440 .83101 Alpha virt. eigenvalues -- 0.832660 .836070 .836450 .836840 .83930 Alpha virt. eigenvalues -- 0.839410 .840410 .842340 .843680 .84551 Alpha virt. eigenvalues -- 0.846440 .846500 .847780 .848560 .85077 Alpha virt. eigenvalues -- 0.853560 .856220 .859540 .860360 .86200 Alpha virt. eigenvalues -- 0.868840 .872940 .874120 .877040 .87963 Alpha virt. eigenvalues -- 0.883520 .884760 .888360 .890690 .89461 Alpha virt. eigenvalues -- 0.901250 .904900 .910810 .912580 .91435 Alpha virt. eigenvalues -- 0.918480 .935430 .938060 .946150 .94695 Alpha virt. eigenvalues -- 0.948230 .953290 .958940 .960840 .96822 Alpha virt. eigenvalues -- 0.971890 .978550 .990420 .992270 .99371 Alpha virt. eigenvalues -- 0.997710 .998251 .006421 .018521 .01974 Alpha virt. eigenvalues -- 1.022481 .029321 .030931 .032681 .03332 Alpha virt. eigenvalues -- 1.037191 .038231 .047291 .049511 .05950 Alpha virt. eigenvalues -- 1.061841 .069901 .073181 .082551 .08331 Alpha virt. eigenvalues -- 1.084301 .084851 .091111 .093091 .09781 Alpha virt. eigenvalues -- 1.098281 .099421 .101481 .106981 .10919 Alpha virt. eigenvalues -- 1.113691 .116091 .116201 .122091 .12578 Alpha virt. eigenvalues -- 1.127721 .131371 .131621 .135501 .13796 Alpha virt. eigenvalues -- 1.138561 .139621 .150131 .152171 .15395 Alpha virt. eigenvalues -- 1.154521 .160111 .162921 .163961 .17191 Alpha virt. eigenvalues -- 1.182821 .184351 .190711 .193471 .19855 Alpha virt. eigenvalues -- 1.200721 .211501 .219341 .229841 .23590 Alpha virt. eigenvalues -- 1.241911 .254491 .257971 .266191 .27916 Alpha virt. eigenvalues -- 1.282871 .287661 .288791 .294931 .29977 Alpha virt. eigenvalues -- 1.310891 .314191 .316281 .329801 .33774 Alpha virt. eigenvalues -- 1.339321 .343861 .351311 .351461 .36837 Alpha virt. eigenvalues -- 1.371051 .371911 .375841 .385491 .39558 Alpha virt. eigenvalues -- 1.396071 .419981 .423601 .439521 .44352 Alpha virt. eigenvalues -- 1.451531 .469111 .478101 .501531 .53425 Alpha virt. eigenvalues -- 1.578891 .593791 .601041 .601211 .60339 Alpha virt. eigenvalues -- 1.606691 .610301 .620001 .741211 .80846 Alpha virt. eigenvalues -- 1.831311 .89007

\section{Chloroform}

Alpha occ. eigenvalues -- -19.21674 -19.21674 -14.34081 -14.34080 -10.29353 Alpha occ. eigenvalues -- -10.29352 -10.26108 -10.26098 -10.23605 -10.23597 Alpha occ. eigenvalues -- -10.22085 -10.22075 -10.20813 -10.20813 -10.20427 Alpha occ. eigenvalues -- -10.20415 -10.20084 -10.20084 -10.19973 -10.19973 Alpha occ. eigenvalues -- -10.19624 -10.19624 -10.19599 -10.19599 -10.19569 Alpha occ. eigenvalues -- -10.19569 -10.19566 -10.19566 -10.19340 -10.19340 Alpha occ. eigenvalues -- -10.19253 -10.19253 -10.19152 -10.19152 -10.19082 Alpha occ. eigenvalues -- -10.19082 -10.18793 -10.18793 -10.18740 -10.18740 Alpha occ. eigenvalues -- -10.18592 -10.18592-10.18531-10.18531-10.18335 
Alpha occ. eigenvalues -- -10.18335 -10.18286 -10.18286 -10.18137 -10.18137 Alpha occ. eigenvalues -- -10.17994 -10.17994 -10.17987 -10.17987 -10.17944 Alpha occ. eigenvalues -- -10.17944 -10.17875 -10.17875 -10.17736 -10.17736 Alpha occ. eigenvalues -- -10.17687 -10.17687 -10.17676 -10.17676 -10.17599 Alpha occ. eigenvalues -- -10.17599 -10.16170 -10.16170 -10.16168 -10.16168 Alpha occ. eigenvalues -- -10.16026 -10.16026 -10.15836 -10.15836 -10.15834 Alpha occ. eigenvalues -- -10.15834 -10.15811 -10.15811 -10.15804-10.15804 Alpha occ. eigenvalues -- -10.15733 -10.15733 -10.15700 -10.15700 -1.14798 Alpha occ. eigenvalues -- -1.14691 -0.95585 -0.95583 -0.90079 -0.88628 Alpha occ. eigenvalues -- -0.88383 -0.88296 -0.87578 -0.87108 -0.87107 Alpha occ. eigenvalues -- -0.86295 -0.85876 -0.83505 -0.82984 -0.82606 Alpha occ. eigenvalues -- -0.82597 -0.82581 -0.81849 -0.80780 -0.79485 Alpha occ. eigenvalues -- $-0.78140-0.78128-0.78124-0.76837-0.76156$ Alpha occ. eigenvalues -- $-0.76148-0.75288-0.75047-0.75012-0.74984$ Alpha occ. eigenvalues -- -0.74850 -0.73836 -0.73163-0.72351 -0.71973 Alpha occ. eigenvalues -- -0.71891 -0.69205-0.68798 -0.68731 -0.68553 Alpha occ. eigenvalues -- -0.68553-0.68419-0.68412 -0.68377 -0.68375 Alpha occ. eigenvalues -- -0.68216 -0.68216 -0.68186 -0.68186 -0.66555 Alpha occ. eigenvalues -- -0.65690 -0.64794 -0.64327 -0.63926 -0.63918 Alpha occ. eigenvalues -- -0.63007 -0.62747 -0.61394-0.61320 -0.61138 Alpha occ. eigenvalues -- -0.60929 -0.60878 -0.60554-0.59822 -0.59244 Alpha occ. eigenvalues -- $-0.58523-0.58336-0.57736-0.56712-0.56314$ Alpha occ. eigenvalues -- -0.56117 -0.55899-0.55520 -0.55505 -0.55434 Alpha occ. eigenvalues -- -0.55433 -0.54232 -0.53678 -0.53203-0.50935 Alpha occ. eigenvalues -- -0.50342 -0.50128 -0.50116 -0.49976 -0.49903 Alpha occ. eigenvalues -- $-0.49691-0.49110-0.48900-0.48850-0.48478$ Alpha occ. eigenvalues -- -0.48019 -0.47823 -0.47749 -0.47208 -0.47206 Alpha occ. eigenvalues -- -0.46833 -0.46544-0.46130 -0.46057 -0.45594 Alpha occ. eigenvalues -- $-0.45575-0.45521-0.45327-0.45320-0.45194$ Alpha occ. eigenvalues -- -0.45020 -0.44850-0.44824-0.44772 -0.44741 Alpha occ. eigenvalues -- -0.44733 -0.44448 -0.44446 -0.44414-0.44409 Alpha occ. eigenvalues -- -0.44094 -0.44068 -0.43179-0.43118 -0.43080 Alpha occ. eigenvalues -- $-0.43059-0.43003-0.42930-0.42475-0.42471$ Alpha occ. eigenvalues -- -0.42417 -0.42407 -0.41632 -0.41053-0.40593 Alpha occ. eigenvalues -- $-0.40550-0.40421-0.40363-0.40260-0.40141$ Alpha occ. eigenvalues -- -0.39952 -0.39940 -0.39893-0.39868 -0.39655 Alpha occ. eigenvalues -- -0.39653 -0.39546-0.39516-0.38831 -0.38457 Alpha occ. eigenvalues -- -0.38261 -0.38260 -0.37996 -0.37983 -0.37955 Alpha occ. eigenvalues -- $-0.37937-0.37581-0.37532-0.37252-0.37163$ Alpha occ. eigenvalues -- $-0.37072-0.37006-0.36983-0.36939-0.36745$ Alpha occ. eigenvalues -- $-0.36729-0.36719-0.36678-0.36672-0.36507$ Alpha occ. eigenvalues -- -0.36344 -0.36322 -0.35898 -0.35856-0.35233 Alpha occ. eigenvalues -- -0.35219 -0.35155 -0.35044-0.34678 -0.34159 Alpha occ. eigenvalues -- -0.33773 -0.33764 -0.33753-0.33750 -0.33628 Alpha occ. eigenvalues -- $-0.33563-0.33425-0.33108-0.33106-0.32898$ Alpha occ. eigenvalues -- $-0.32883-0.32802-0.32791-0.32250-0.32241$ Alpha occ. eigenvalues -- $-0.32178-0.32176-0.30662-0.30164-0.30027$ Alpha occ. eigenvalues -- -0.29443 -0.29227 -0.29140 -0.28259-0.27467 Alpha occ. eigenvalues -- -0.27467 -0.26487 -0.26484 -0.26423 -0.26422 Alpha occ. eigenvalues -- -0.26160 -0.25238 -0.24127 -0.23709 -0.23132 Alpha occ. eigenvalues -- $-0.23105-0.22311$

Alpha virt. eigenvalues -- -0.09246 -0.05872 -0.05390 -0.04739 -0.04714 Alpha virt. eigenvalues -- -0.03631 -0.02333-0.02268 -0.02125 -0.01599 Alpha virt. eigenvalues -- -0.01563 -0.01514-0.01492 -0.00048 0.00359 Alpha virt. eigenvalues -- 0.024370 .024520 .028280 .036570 .04950 Alpha virt. eigenvalues -- 0.050850 .051910 .056950 .057540 .05857 
Alpha virt. eigenvalues -- 0.058760 .063570 .065920 .070360 .07093 Alpha virt. eigenvalues -- 0.085150 .085580 .087620 .089840 .09043 Alpha virt. eigenvalues -- 0.093790 .097550 .098470 .103430 .10363 Alpha virt. eigenvalues -- 0.103660 .104940 .105390 .105690 .10937 Alpha virt. eigenvalues -- 0.109380 .109650 .112250 .112570 .11423 Alpha virt. eigenvalues -- 0.114980 .115660 .116270 .116610 .11764 Alpha virt. eigenvalues -- 0.117690 .118450 .118590 .119320 .12008 Alpha virt. eigenvalues -- 0.125800 .125850 .127000 .127030 .12886 Alpha virt. eigenvalues -- 0.129280 .131520 .131970 .132710 .13588 Alpha virt. eigenvalues -- 0.138340 .140570 .141360 .142560 .14597 Alpha virt. eigenvalues -- 0.148540 .150560 .151140 .153600 .15378 Alpha virt. eigenvalues -- 0.156300 .157110 .159310 .162040 .16287 Alpha virt. eigenvalues -- 0.166030 .168240 .168760 .170080 .17262 Alpha virt. eigenvalues -- 0.173620 .173630 .175180 .175590 .17581 Alpha virt. eigenvalues -- 0.175850 .176900 .177350 .179050 .18089 Alpha virt. eigenvalues -- 0.184680 .187350 .188890 .188910 .19050 Alpha virt. eigenvalues -- 0.191010 .191080 .191940 .192160 .19249 Alpha virt. eigenvalues -- 0.192920 .194040 .194730 .195750 .19681 Alpha virt. eigenvalues -- 0.199390 .204190 .204400 .205610 .20610 Alpha virt. eigenvalues -- 0.208140 .208280 .212000 .215830 .21592 Alpha virt. eigenvalues -- 0.218770 .221530 .231390 .236060 .23718 Alpha virt. eigenvalues -- 0.237740 .238810 .239140 .240300 .24086 Alpha virt. eigenvalues -- 0.244230 .244410 .244800 .247800 .24819 Alpha virt. eigenvalues -- 0.248200 .248450 .254070 .256280 .25933 Alpha virt. eigenvalues -- 0.260770 .264930 .267550 .267610 .26836 Alpha virt. eigenvalues -- 0.271040 .271750 .274950 .276710 .27894 Alpha virt. eigenvalues -- 0.279450 .281280 .284330 .285040 .28940 Alpha virt. eigenvalues -- 0.290710 .295290 .295310 .296620 .30196 Alpha virt. eigenvalues -- 0.303790 .304410 .309550 .310900 .31137 Alpha virt. eigenvalues -- 0.312500 .313360 .316910 .322790 .32388 Alpha virt. eigenvalues -- 0.326200 .328050 .330650 .332600 .33401 Alpha virt. eigenvalues -- 0.336420 .336900 .337890 .338090 .34353 Alpha virt. eigenvalues -- 0.355920 .357070 .357320 .358040 .35982 Alpha virt. eigenvalues -- 0.363660 .365630 .371040 .375840 .37672 Alpha virt. eigenvalues -- 0.379270 .386610 .387450 .389020 .39033 Alpha virt. eigenvalues -- 0.391910 .396190 .404940 .405170 .40628 Alpha virt. eigenvalues -- 0.407670 .413930 .417180 .418270 .41829 Alpha virt. eigenvalues -- 0.421240 .421240 .421550 .421550 .42572 Alpha virt. eigenvalues -- 0.425790 .429430 .432250 .433240 .43537 Alpha virt. eigenvalues -- 0.435730 .436980 .437100 .438220 .44035 Alpha virt. eigenvalues -- 0.442040 .442300 .442870 .443120 .44581 Alpha virt. eigenvalues -- 0.448100 .453600 .454850 .457780 .45827 Alpha virt. eigenvalues -- 0.459920 .461060 .461650 .462700 .46318 Alpha virt. eigenvalues -- 0.463650 .465360 .466670 .466680 .47472 Alpha virt. eigenvalues -- 0.475550 .477970 .479040 .479160 .48118 Alpha virt. eigenvalues -- 0.481230 .481940 .482590 .483170 .48773 Alpha virt. eigenvalues -- 0.490640 .492600 .493760 .496500 .49849 Alpha virt. eigenvalues -- 0.501610 .502090 .503190 .504080 .50563 Alpha virt. eigenvalues -- 0.510990 .511250 .513170 .513670 .51590 Alpha virt. eigenvalues -- 0.519120 .520390 .520540 .522910 .53509 Alpha virt. eigenvalues -- 0.535810 .542460 .543950 .545830 .54713 Alpha virt. eigenvalues -- 0.548140 .554090 .556290 .556670 .55971 Alpha virt. eigenvalues -- 0.564530 .566030 .567430 .570210 .57117 Alpha virt. eigenvalues -- 0.575660 .575690 .576730 .578240 .58138 Alpha virt. eigenvalues -- 0.582650 .582890 .585000 .588280 .58869 Alpha virt. eigenvalues -- 0.591600 .594490 .595470 .600030 .60321 
Alpha virt. eigenvalues -- 0.604890 .605300 .612670 .613880 .61586 Alpha virt. eigenvalues -- 0.617220 .618910 .619700 .620660 .62149 Alpha virt. eigenvalues -- 0.622770 .623150 .624420 .624590 .62707 Alpha virt. eigenvalues -- 0.629950 .632180 .632700 .632880 .63447 Alpha virt. eigenvalues -- 0.635240 .644590 .646840 .647150 .64865 Alpha virt. eigenvalues -- 0.650240 .650250 .653570 .657360 .65951 Alpha virt. eigenvalues -- 0.660440 .663040 .663970 .666360 .66761 Alpha virt. eigenvalues -- 0.670160 .670400 .670880 .671170 .67179 Alpha virt. eigenvalues -- 0.671890 .673270 .673280 .674420 .67446 Alpha virt. eigenvalues -- 0.675780 .676590 .677110 .678590 .67955 Alpha virt. eigenvalues -- 0.679590 .681500 .683950 .684300 .68589 Alpha virt. eigenvalues -- 0.686390 .686890 .688520 .689220 .69006 Alpha virt. eigenvalues -- 0.691840 .692260 .692990 .694290 .69551 Alpha virt. eigenvalues -- 0.695900 .696900 .697000 .697860 .69810 Alpha virt. eigenvalues -- 0.699580 .700630 .700770 .702880 .70392 Alpha virt. eigenvalues -- 0.704520 .705370 .706460 .706670 .70776 Alpha virt. eigenvalues -- 0.708190 .708880 .709960 .712380 .71298 Alpha virt. eigenvalues -- 0.713970 .716810 .717520 .722190 .72329 Alpha virt. eigenvalues -- 0.723350 .723750 .724070 .724780 .72569 Alpha virt. eigenvalues -- 0.727100 .727550 .727870 .728890 .73024 Alpha virt. eigenvalues -- 0.731250 .731920 .732680 .733740 .73424 Alpha virt. eigenvalues -- 0.735220 .735910 .736860 .738020 .73861 Alpha virt. eigenvalues -- 0.741240 .743100 .744130 .745460 .74645 Alpha virt. eigenvalues -- 0.747510 .748680 .749130 .750140 .75045 Alpha virt. eigenvalues -- 0.752840 .753110 .755500 .756270 .75640 Alpha virt. eigenvalues -- 0.756750 .757690 .757800 .758860 .76286 Alpha virt. eigenvalues -- 0.764140 .765440 .766690 .768300 .76910 Alpha virt. eigenvalues -- 0.771790 .774040 .774470 .778260 .77893 Alpha virt. eigenvalues -- 0.779740 .780280 .782820 .783780 .78902 Alpha virt. eigenvalues -- 0.789420 .792800 .793490 .795150 .79632 Alpha virt. eigenvalues -- 0.798980 .799750 .800800 .803180 .80322 Alpha virt. eigenvalues -- 0.806340 .808530 .813480 .814500 .81598 Alpha virt. eigenvalues -- 0.817780 .818100 .819780 .820430 .82439 Alpha virt. eigenvalues -- 0.824870 .830190 .830650 .831070 .83351 Alpha virt. eigenvalues -- 0.835860 .837530 .841430 .843720 .84498 Alpha virt. eigenvalues -- 0.845670 .845790 .846760 .847570 .84809 Alpha virt. eigenvalues -- 0.848710 .850530 .853610 .855240 .85570 Alpha virt. eigenvalues -- 0.861040 .864390 .865470 .868160 .87266 Alpha virt. eigenvalues -- 0.876700 .877670 .879860 .882950 .89212 Alpha virt. eigenvalues -- 0.893210 .900850 .903240 .906820 .90919 Alpha virt. eigenvalues -- 0.911240 .924420 .927850 .937530 .93920 Alpha virt. eigenvalues -- 0.940340 .946090 .949600 .954870 .96084 Alpha virt. eigenvalues -- 0.967690 .970520 .985500 .986860 .98803 Alpha virt. eigenvalues -- 0.988870 .993651 .000151 .013421 .01369 Alpha virt. eigenvalues -- 1.017371 .023511 .024561 .028501 .02986 Alpha virt. eigenvalues -- 1.031251 .031401 .043361 .045241 .05278 Alpha virt. eigenvalues -- 1.054101 .063421 .066181 .078381 .07898 Alpha virt. eigenvalues -- 1.079981 .081151 .083841 .087491 .09201 Alpha virt. eigenvalues -- 1.093861 .094041 .096301 .103921 .10452 Alpha virt. eigenvalues -- 1.108311 .111411 .112201 .122631 .12417 Alpha virt. eigenvalues -- 1.126331 .128641 .129971 .130201 .13392 Alpha virt. eigenvalues -- 1.137271 .137441 .142051 .145501 .14696 Alpha virt. eigenvalues -- 1.148141 .151661 .154611 .156621 .16253 Alpha virt. eigenvalues -- 1.173001 .177911 .178191 .183301 .18879 Alpha virt. eigenvalues -- 1.190481 .204451 .208301 .218591 .23132 Alpha virt. eigenvalues -- 1.236111 .248361 .252051 .261971 .27496 
Alpha virt. eigenvalues -- 1.280661 .282511 .282521 .290461 .29422

Alpha virt. eigenvalues -- 1.303631 .306751 .311771 .323691 .33191

Alpha virt. eigenvalues -- 1.331971 .337541 .343751 .343961 .36007

Alpha virt. eigenvalues -- 1.363281 .367081 .369351 .381431 .38938

Alpha virt. eigenvalues -- 1.390131 .412851 .415691 .430851 .43537

Alpha virt. eigenvalues -- 1.441221 .461391 .467621 .494551 .52812

Alpha virt. eigenvalues -- 1.572571 .585241 .595301 .596401 .59830

Alpha virt. eigenvalues -- 1.601101 .603391 .614381 .731301 .80102

Alpha virt. eigenvalues -- 1.823321 .88117

\section{Comparison of Experimental and DFT UV-Vis}

\section{Gas Phase}

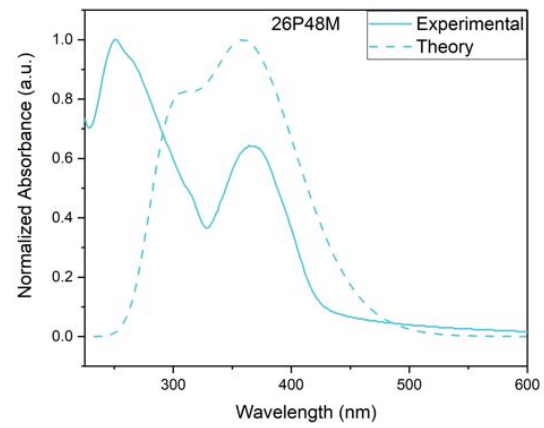

\section{Chloroform}

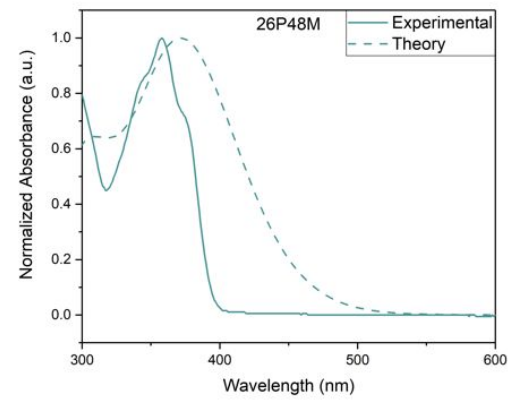

Figure S34. Gas phase and chloroform overlays of computation and experimental data for 26P48M.

\section{Lowest 15 Excited States}

\begin{tabular}{|c|c|c|c|c|c|c|}
\hline \multicolumn{4}{|c|}{ Gas Phase } & \multicolumn{3}{|c|}{ Chloroform } \\
\hline$\frac{\text { Excited }}{\text { state }}$ & $\frac{\text { Energy }}{(\mathrm{eV})}$ & $\frac{\text { Wavelength }}{(\mathrm{nm})}$ & $f$ & $\frac{\text { Energy }}{(\mathrm{eV})}$ & $\frac{\text { Wavelength }}{(\mathrm{nm})}$ & $f$ \\
\hline 1 & 3.17 & 391.62 & 0.60 & 3.18 & 389.71 & 0.96 \\
\hline 2 & 3.27 & 379.48 & 0.00 & 3.33 & 372.80 & 0.01 \\
\hline 3 & 3.28 & 378.39 & 0.00 & 3.34 & 371.76 & 0.01 \\
\hline 4 & 3.53 & 351.58 & 0.95 & 3.45 & 358.95 & 0.98 \\
\hline 5 & 3.62 & 342.52 & 0.00 & 3.65 & 339.82 & 0.00 \\
\hline 6 & 3.90 & 317.88 & 0.02 & 3.92 & 316.29 & 0.04 \\
\hline 7 & 3.90 & 317.86 & 0.00 & 3.99 & 310.66 & 0.00 \\
\hline 8 & 4.12 & 300.54 & 0.68 & 4.10 & 302.59 & 0.90 \\
\hline 9 & 4.15 & 298.56 & 0.00 & 4.16 & 298.06 & 0.00 \\
\hline 10 & 4.17 & 297.45 & 0.24 & 4.18 & 296.27 & 0.07 \\
\hline 11 & 4.21 & 294.60 & 0.00 & 4.19 & 296.23 & 0.00 \\
\hline 12 & 4.22 & 293.59 & 0.02 & 4.29 & 288.93 & 0.01 \\
\hline 13 & 4.23 & 292.82 & 0.00 & 4.30 & 288.47 & 0.01 \\
\hline 14 & 4.27 & 290.08 & 0.00 & 4.30 & 288.29 & 0.00 \\
\hline 15 & 4.28 & 290.01 & 0.00 & 4.30 & 288.08 & 0.00 \\
\hline
\end{tabular}

Table S9. Gas phase and chloroform excitation energies, wavelengths, and oscillator strengths for first 15 excited state of $26 \mathrm{P} 48 \mathrm{M}$. 


\section{Geometric Images}
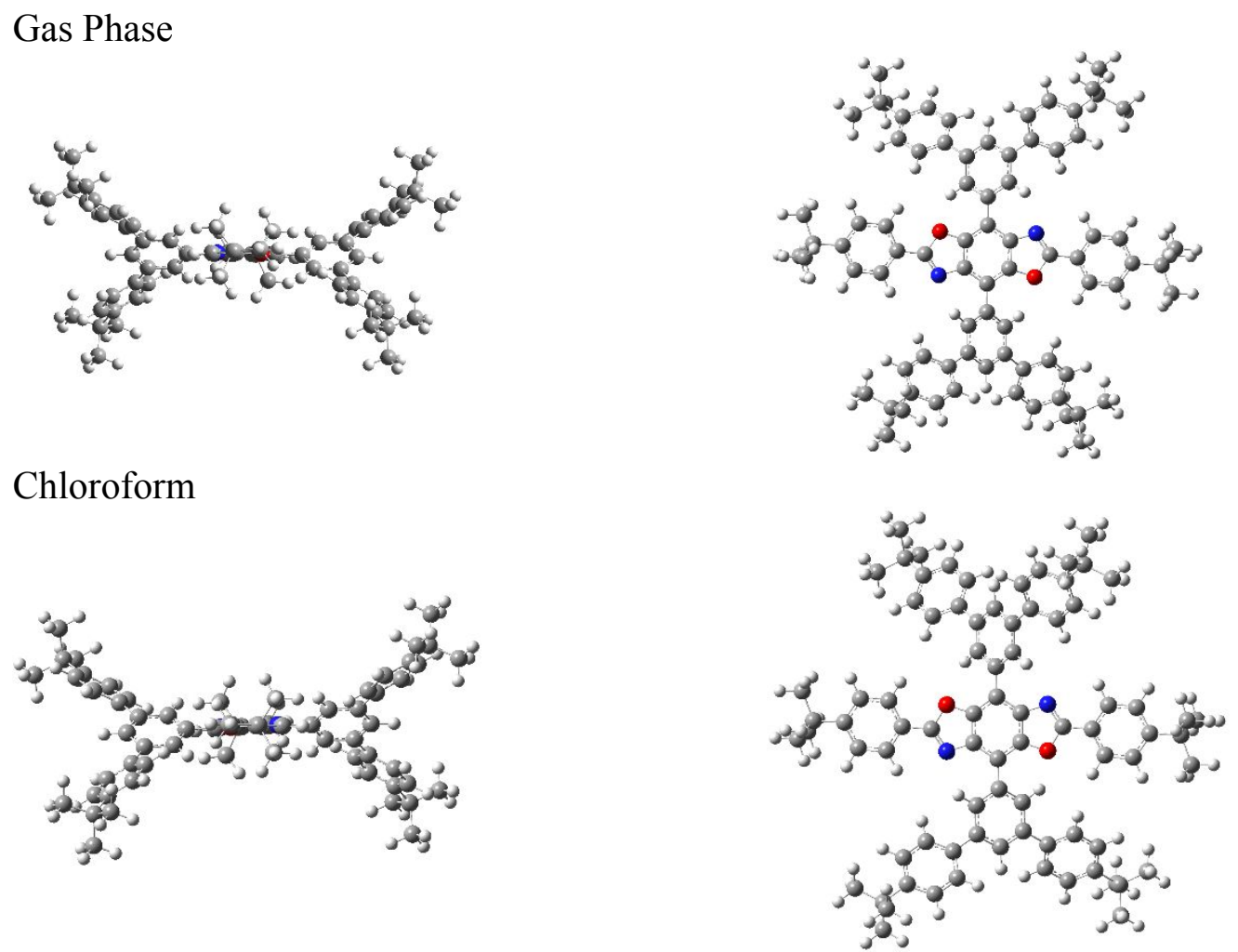

Figure S35. Geometric optimization images in gas phase and chloroform for 26P48M.

\section{M48M}

\section{Cartesian Coordinates}

Gas Phase

C $-0.11958-1.50246-0.22453$

C $-1.19687-0.60842-0.22499$

C $0.11961 .5023-0.22442$

C $1.196870 .60827-0.22493$

C $-1.115730 .81088-0.2089$

C $-3.229450 .29931-0.15135$

O $-2.5551-0.93196-0.19537$

$\mathrm{N}-2.416311 .32717-0.16552$

C $1.11574-0.81104-0.20895$

C $3.22946-0.29945-0.15134$

N $2.41634-1.32731-0.16561$

O $2.55510 .93182-0.19529$

C $0.254252 .97425-0.23003$

C -0.686913 .783590 .437$

C $1.326713 .59109-0.90586$

C -0.560845 .185390 .45137$

H -1.543053 .311120 .92125$

C $1.464334 .99146-0.92784$ 
H $2.069212 .9715-1.41296$

C - 1.548656 .02651 .17637

C $0.518085 .77259-0.23716$

C $2.580065 .63484-1.66955$

C -2.137195 .588242 .37792$

C -1.929937 .297080 .69526$

H $0.622146 .86147-0.24002$

C $3.215486 .79549-1.17943$

C $3.034225 .1195-2.89828$

C -3.061576 .382353 .07032$

$\mathrm{H}-1.852714 .618052 .79654$

C -2.851358 .086411 .38956$

H $-1.518517 .66388-0.25041$

C $4.249987 .40881-1.892$

H $2.910527 .21266-0.21441$

C $4.071435 .73831-3.60984$

H $2.551384 .2345-3.32443$

C -3.44133 7.65178 2.59601

H -3.48012 5.99268 4.00075

H -3.12065 9.06175 0.97147

C $4.703726 .89948-3.12771$

H $4.716348 .30308-1.46604$

H $4.376225 .30043-4.56275$

C $-0.25426-2.97441-0.23015$

C $0.68697-3.783770 .43676$

C $-1.32681-3.59122-0.90585$

C $0.56086-5.185570 .45116$

H $1.54319-3.311310 .92087$

C - $1.46448-4.9916-0.92779$

H - $2.06935-2.97162-1.41289$

C $1.54876-6.026721 .176$

C $-0.51818-5.77274-0.23722$

C - $-2.58032-5.63494-1.66937$

C $2.13762-5.588432 .37737$

C $1.92979-7.297380 .6949$

H $-0.6223-6.86161-0.24003$

C $-3.2157-6.79559-1.17918$

C $-3.03462-5.1196-2.89805$

C $3.06209-6.382583 .06961$

H $1.85333-4.618182 .796$

C $2.85129-8.086751 .38903$

H $1.51809-7.66421-0.25063$

C $-4.25028-7.4089-1.89165$

H -2.91064 -7.21278 - 0.2142

C $-4.07191-5.73839-3.60949$

H - $2.55182-4.23461-3.32426$

C $3.44161-7.652082 .5953$

H $3.4809-5.99293 .99993$

H $3.12038-9.062150 .97097$

C $-4.70415-6.89956-3.1273$

H -4.7166 -8.30317 - 1.46564

H $-4.37679-5.30051-4.56237$

C $-4.679980 .29348-0.06954$

C $-5.359121 .52596-0.02055$

C $-5.397-0.91566-0.01267$

C -6.759661 .562510 .08904$ 
H -4.77118 2.4472 -0.04009

C -6.79998 -0.90626 0.10532

H - $4.85236-1.86095-0.07284$

C -7.489572 .855660 .15136$

C -7.45810 .339040 .1524$

C -7.57354 -2.17262 0.19493

C - $7.043683 .98423-0.56208$

C -8.659742 .999820 .9269$

H -8.547160 .358760 .24648$

C $-8.88575-2.27056-0.31564$

C $-7.03634-3.317050 .8145$

C -7.73767 5.20128 -0.50836

H -6.15079 $3.91065-1.19057$

C -9.348674 .214710 .97684$

H -9.023982 .157131 .52288$

C $-9.6257-3.44948-0.18999$

H -9.33075 - $1.41793-0.83786$

C $-7.7798-4.499410 .93287$

H -6.02891 -3.28303 1.24024

C -8.908585 .347610 .25906$

H -7.35018 6.0412 -1.08886

H - 10.247134 .278191 .5991

C $-9.09606-4.59450 .44302$

H $-10.63991-3.473-0.60105$

H - $7.31342-5.352251 .43039$

C $4.67999-0.29357-0.06949$

C $5.35921-1.526-0.02043$

C $5.396930 .91562-0.01267$

C $6.75975-1.562450 .08919$

H $4.77134-2.44728-0.0399$

C 6.799920 .906320 .10534

H $4.852261 .86089-0.07291$

C $7.48977-2.855550 .15159$

C $7.45811-0.338930 .15249$

C 7.573392 .172730 .1949

C $7.04398-3.9842-0.56179$

C $8.65993-2.999550 .92715$

H $8.54718-0.358580 .24656$

C $8.885552 .27078-0.31578$

C 7.036143 .317110 .81453

C $7.73809-5.20118-0.50799$

H $6.15109-3.91073-1.1903$

C $9.34898-4.214380 .97717$

H 9.02409-2.1568 1.5231

C $9.625413 .44976-0.19017$

H $9.330561 .41818-0.83804$

C 7.779534 .499520 .93286

H 6.028753 .2831 .24035

C $8.909-5.347350 .25944$

H 7.35068 -6.04117 -1.08845

H $10.24743-4.277751 .59944$

C 9.095734 .594730 .4429

H $10.63963 .47336-0.60131$

H 7.31312 5.35232 1.43043

C $-5.84545-7.60542-3.88043$

C -7.12387 -7.57965 -3.00581 
C -6.16589 -6.9194 -5.22366

C $-5.44621-9.07406-4.16891$

H -6.96778 -8.09377 -2.03937

H -7.4273 -6.53832 -2.78996

H -7.95925 -8.08469 -3.52865

H -5.29195 -6.91907 -5.90162

H -6.98472 -7.46156 -5.73309

H -6.49664 -5.87391 -5.08055

H -6.25976 -9.59286 -4.71217

H $-4.53218-9.11827-4.79051$

H -5.24998 -9.63664 -3.23773

C $4.44922-8.555623 .32796$

C $5.64391-8.860192 .3899$

C $4.99811-7.893974 .60779$

C $3.75462-9.882523 .72481$

H $5.32112-9.385141 .47209$

H $6.15058-7.924972 .08582$

H $6.38289-9.503982 .90543$

H $4.1938-7.679615 .33603$

H $5.71987-8.573865 .09836$

H $5.52681-6.948624 .38431$

H $4.46749-10.548084 .24941$

H $2.89916-9.690984 .39953$

H $3.37294-10.424312 .83992$

C -4.448778 .555323 .32889$

C -5.643548 .86022 .39104$

C -4.997597 .893554 .60869$

C -3.753919 .882073 .72584$

H -5.32083 9.38521 .47323

H -6.150427 .92512 .08693$

H -6.38233 9.50405 2.90676

H -4.19325 7.67912 5.33686

H -5.71931 8.57341 5.09938

H -5.52631 6.94824 4.38514

H -4.46659 10.54767 4.25064

H -2.898389.69029 4.40039

H -3.37228 10.42392 .84095

C $5.844937 .60536-3.88095$

C $7.123427 .57964-3.00644$

C $6.165276 .91935-5.22421$

C $5.445629 .07399-4.16938$

H $6.967428 .09378-2.04001$

H $7.426896 .53831-2.79059$

H $7.958758 .08466-3.52937$

H $5.291316 .91907-5.90212$

H $6.984117 .46148-5.73366$

H $6.495995 .87384-5.08112$

H $6.25919 .59283-4.71271$

H $4.531539 .11818-4.7909$

H $5.249469 .63655-3.23818$

C $9.7048-6.662460 .33627$

C $9.78063-7.134841 .80985$

C $9.05492-7.78426-0.4983$

C $11.13878-6.42491-0.20127$

H $10.27971-6.388452 .45456$

H $8.76815-7.313632 .2176$ 
H $10.35407-8.079431 .87979$

H $9.0042-7.51992-1.571$

H $9.65589-8.70888-0.41069$

H $8.03201-8.01558-0.14697$

H $11.72666-7.3619-0.15158$

H $11.11276-6.08797-1.25459$

H 11.67408 -5.6575 0.38748

C 9.958415 .860280 .59527

C $10.472756 .31857-0.79185$

C 9.17043 7.02619 1.2255

C 11.168615 .536981 .50836

H $11.092265 .54344-1.27853$

H $9.628746 .55409-1.46655$

H $11.095537 .2278-0.68549$

H 8.819586 .781432 .24523

H 9.821897 .916911 .30324

H 8.292737 .303690 .61195

H 11.808496 .432581 .62952

H 10.829945 .214742 .51091

H 11.791714 .727851 .08439

C $-9.95883-5.859990 .59546$

C $-10.4733-6.31826-0.79161$

C $-9.17089-7.025951 .22565$

C $-11.16893-5.536591 .50863$

H -11.09279 -5.5431 -1.27826

H $-9.62935-6.55385-1.46637$

H - $11.09612-7.22745-0.6852$

H -8.81993 -6.78119 2.24534

H -9.82241 -7.91661 1.30347

H -8.29326 -7.30353 0.61203

H - $11.80887-6.432131 .62985$

H - $10.83017-5.214342 .51115$

H -11.79201 -4.72741 1.08469

C -9.704246 .662810 .33581$

C -9.779987 .13531 .80935$

C $-9.054267 .78448-0.49887$

C - $11.138266 .42536-0.20169$

H - 10.279136 .3892 .45413

H -8.76748 7.31401 2.21707

H - 10.353328 .079951 .87925

H -9.00353 7.52 -1.57153

H -9.65519 8.70914 -0.41139

H -8.03135 8.01579 -0.14754

H - $11.726047 .36241-0.15201$

H - $11.11236 .08838-1.25498$

H -11.67361 5.658030 .38712

Chloroform

C -1.139410 .761680 .01927$

C $-1.17734-0.65840 .03656$

C $-0.07108-1.514240 .04756$

C $1.14274-0.787970 .02979$

C 1.180270 .632340 .0282

C 0.07421 .487870 .02622

C $-3.237030 .18626-0.01434$ 
O - $2.52479-1.024130 .01405$

$\mathrm{N}-2.455161 .23948-0.00531$

O 2.527070 .998670 .00118

N $2.45908-1.265050 .01113$

C $3.24022-0.21155-0.01178$

C 0.153842 .964170 .04187

C $-0.74423 .73467-0.72184$

C 1.118693 .618310 .83315

C $-0.68965 .14175-0.70624$

H - $1.476443 .22588-1.35119$

C 1.190565 .024730 .87831

H 1.792793 .021581 .45193

C 0.281815 .768570 .09934

H 0.329696 .860830 .12214

C - $1.636025 .94488-1.52477$

C $-1.254867 .17254-2.10043$

C $-2.957335 .5033-1.7552$

C - $2.152797 .92812-2.86895$

H $-0.232187 .54006-1.97054$

C $-3.848886 .25974-2.52238$

H $-3.300754 .5647-1.30845$

C $-3.472267 .49293-3.09891$

H - 1.799428 .86866 -3.29695

H -4.8644 $5.87745-2.66712$

C - $4.486448 .29252-3.93573$

C $-4.941187 .4375-5.14557$

C -5.71781 8.63818 -3.06031

C $-3.888559 .60947-4.46973$

H $-4.08027 .18011-5.79103$

H -5.42058 6.49485 -4.8233

H -5.67456 7.99935 -5.75568

H -5.4217 9.25424 -2.19031

H -6.45753 9.21017 -3.65297

$\mathrm{H}-6.220067 .72884-2.681$

H -4.65172 $10.15084-5.05962$

H -3.56563 10.2757 -3.64796

H -3.02138 9.42757 -5.13169

C 2.192545 .708241 .7388

C 3.457455 .138721 .98275

C 1.91726 .952952 .34635

C 4.408055 .783972 .78718

H 3.7184 .181791 .52014

C 2.867647 .593253 .14808

H 0.936977 .420542 .21169

C 4.140147 .029313 .38763

H 5.374835 .29722 .93438

H 2.601638 .553453 .60133

C 5.157437 .770624 .27266

C 6.490237 .004994 .39149

C 5.450169 .164813 .66287

C 4.566697 .946115 .69463

H 6.351496 .007664 .84861

H 6.976256 .872543 .40636

H 7.18786 7.57302 5.03497

H 4.53559 .781683 .59136

H 6.178359 .711114 .29295 
H 5.877429 .067722 .64681

H 5.286058 .482536 .343

H 3.626488 .527555 .67804

H 4.352846 .963246 .15542

C - $0.15216-2.989810 .08953$

C $0.75845-3.77702-0.64183$

C - $1.13212-3.627090 .87652$

C $0.69919-5.18332-0.5999$

H $1.5047-3.28327-1.26669$

C - $1.20733-5.032040 .94895$

H - $1.81581-3.017371 .47152$

C $-0.28798-5.792910 .19947$

H $-0.33921-6.884540 .24127$

C $1.65637-6.00426-1.38739$

C $1.27057-7.22698-1.97009$

C $2.99096-5.58535-1.58046$

C $2.17614-7.99804-2.71372$

H $0.23867-7.57686-1.86644$

C $3.89072-6.35816-2.32143$

H $3.33797-4.65182-1.1257$

C $3.50815-7.58454-2.90879$

H $1.81894-8.93314-3.15041$

H 4.91677 -5.99422 -2.43476

C $-2.22132-5.695831 .81063$

C $-3.49208-5.1192 .02442$

C $-1.95443-6.921462 .4519$

C $-4.44595-5.744212 .8338$

H - $3.74736-4.174691 .53361$

C $-2.91342-7.545153 .26363$

H - $0.97287-7.392552 .34064$

C -4.18379-6.97527 3.47474

H -5.4189 -5.25822 2.95945

H - $2.64656-8.490673 .74092$

C $4.52849-8.39986-3.72241$

C $5.01515-7.55429-4.92658$

C $5.73914-8.75594-2.82331$

C $3.9255-9.71196-4.26267$

H 4.16924 -7.29043 -5.58921

H $5.49768-6.61541-4.59801$

H $5.75416-8.12586-5.5208$

H $5.41975-9.36353-1.95557$

H $6.48216-9.34041-3.39951$

H $6.24574-7.8514-2.43944$

H $4.69421-10.26556-4.83377$

H $3.57752-10.36987-3.44438$

H $3.07466-9.52325-4.94361$

C $-5.26193-7.632394 .35423$

C -5.64494 -6.66917 5.50592

C -6.51612 -7.92803 3.49307

C $-4.776-8.95854 .97255$

H -4.76594 -6.44451 6.13918

H -6.04486 -5.71219 5.12361

H -6.42251 -7.12972 6.14531

H -6.27052 -8.62042 2.66569

H -7.30303 -8.39806 4.11403

H -6.93818 -7.0055 3.05321 
H -5.57951 -9.39342 5.59604

H -4.51574 -9.70168 4.19595

H -3.89257 -8.80842 5.62081

C $-4.688760 .13836-0.0588$

C -5.36936-1.08063-0.23135

C -5.409791 .341970 .05732$

C $-6.77632-1.10849-0.29211$

H $-4.79279-2.00078-0.35147$

C -6.814581 .341440 .00345$

H -4.85552 .270420 .21514$

C -7.47767 0.10851 -0.17125

H $-8.57020 .09694-0.21555$

C $4.69184-0.16034-0.05368$

C $5.367981 .06545-0.19204$

C $5.41672-1.365140 .01971$

C $6.77461 .10068-0.25072$

H $4.787811 .98667-0.28249$

C $6.82153-1.35807-0.0332$

H $4.86308-2.3040 .10035$

C $7.48019-0.11673-0.16337$

H $8.57334-0.09458-0.1538$

C -7.579182 .609160 .13611$

C $-7.068823 .82389-0.37052$

C -8.834192 .649920 .77393$

C $-7.783255 .01853-0.24105$

H $-6.107613 .83343-0.89372$

C -9.547683 .8510 .90126$

H -9.257451 .736981 .20456$

C -9.041715 .06530 .39828$

H -7.3452 $5.9321-0.65557$

$\mathrm{H}-10.51193 .82521 .41353$

C $-7.50465-2.38904-0.4911$

C $-8.70804-2.44568-1.22094$

C $-7.01501-3.599180 .0455$

C $-9.39299-3.65594-1.40408$

H $-9.11151-1.53727-1.67898$

C $-7.70046-4.8035-0.13959$

H $-6.09478-3.598430 .63724$

C $-8.90868-4.86496-0.86832$

$\mathrm{H}-10.31715-3.64139-1.98579$

H -7.28058 -5.71205 0.30344

C -9.790376 .403480 .52461$

C -8.930467 .393181 .35102$

C - $10.035586 .9921-0.88782$

C -11.154016 .24171 .22538$

$\mathrm{H}-8.742366 .997072 .36678$

H -7.95262 7.583690 .87162

H -9.45447 8.36341 .44995

H - $10.650476 .30485-1.49916$

H - $10.571047 .95787-0.81023$

H -9.08761 7.1749 -1.42691

H - 11.657287 .224561 .28845

H - 11.821845 .558290 .66831

H - 11.042755 .857052 .25637

C $-9.63204-6.21125-1.04564$

C -8.69286 -7.20737-1.77194 
C - $10.00505-6.77910 .34735$

C $-10.92441-6.0708-1.87429$

H $-8.41471-6.82689-2.77293$

H -7.76192 -7.38358 -1.20218

H $-9.19985-8.18288-1.902$

H $-10.68328-6.089080 .88412$

H - $10.51987-7.752890 .23682$

H -9.11049 -6.93818 0.97727

H - $11.4089-7.05995-1.97554$

H -11.64982 -5.39079 -1.38993

H - $10.71837-5.69299-2.89315$

C $7.498812 .3906-0.3952$

C $8.710732 .47729-1.10783$

C 6.996623 .57990 .17524

C $9.391373 .69628-1.24288$

H 9.12447 1.58626 -1.59026

C 7.677164 .793190 .03727

H 6.069913 .554670 .75613

C $8.893634 .88466-0.67417$

H $10.322833 .70549-1.81297$

H 7.24719 5.68505 0.5039

C $7.5951-2.624240 .05559$

C $7.13486-3.713130 .82776$

C $8.8172-2.78502-0.62616$

C $7.86903-4.900420 .91193$

H $6.2016-3.625511 .39255$

C $9.55034-3.9772-0.5378$

H $9.19873-1.97756-1.25884$

C $9.09744-5.064320 .2343$

H $7.47408-5.712221 .53072$

H 10.48795 -4.04722 -1.09329

C $9.609816 .24054-0.79966$

C 9.952986 .771430 .61541

C $8.675557 .2496-1.51437$

C $10.918256 .1335-1.60777$

H 10.628376 .072491 .14416

H 10.460247 .752650 .54203

H 9.046516 .904851 .23453

H $8.418266 .89538-2.53046$

H $7.733287 .40174-0.95637$

H 9.17651 8.23241 -1.60768

H $11.398047 .12813-1.66945$

H $11.638795 .44311-1.13092$

H $10.734035 .78642-2.64165$

C $9.87634-6.385630 .35922$

C $10.22256-6.638381 .84845$

C 8.99999 -7.54925 -0.16894

C $11.19056-6.36266-0.44628$

H $10.84878-5.819882 .251$

H $9.31391-6.713792 .47375$

H $10.78459-7.586481 .95279$

H $8.73833-7.39328-1.23265$

H $9.54716-8.508-0.08488$

H $8.05898-7.645110 .40329$

H $11.71633-7.32816-0.32477$

H $11.00652-6.21573$-1.52694 
H $11.87136-5.56425-0.0966$

\section{Energy Levels}

\section{Gas Phase}

Alpha occ. eigenvalues -- -19.20874 -19.20874 -14.33194 -14.33193-10.28479

Alpha occ. eigenvalues -- -10.28478 -10.25291 -10.25280 -10.22754 -10.22745

Alpha occ. eigenvalues -- -10.21243 -10.21232 -10.20389 -10.20389 -10.19621

Alpha occ. eigenvalues -- -10.19621 -10.19606 -10.19606 -10.19602 -10.19602

Alpha occ. eigenvalues -- -10.19534 -10.19534 -10.19406 -10.19406 -10.19373

Alpha occ. eigenvalues -- -10.19361 -10.19361 -10.19360 -10.18799 -10.18799

Alpha occ. eigenvalues -- -10.18753 -10.18753 - 10.18578 -10.18578 -10.18562

Alpha occ. eigenvalues -- -10.18562 -10.18496 -10.18496 -10.18496 -10.18496

Alpha occ. eigenvalues -- -10.18478 -10.18478 -10.18380 -10.18380 - 10.18373

Alpha occ. eigenvalues -- -10.18373 -10.18325 -10.18325 -10.18207 -10.18207

Alpha occ. eigenvalues -- -10.18144 -10.18144 -10.18058 -10.18058 -10.17714

Alpha occ. eigenvalues -- -10.17714 -10.17702 -10.17702 -10.17567 -10.17567

Alpha occ. eigenvalues -- -10.17538 -10.17538 -10.17485 -10.17485 -10.17468

Alpha occ. eigenvalues -- -10.17468 -10.17348 -10.17348 -10.17324 -10.17324

Alpha occ. eigenvalues -- -10.17321 -10.17320 -10.17318 -10.17318 -10.17316

Alpha occ. eigenvalues -- -10.17316 -10.17214 -10.17214 -10.17202 -10.17202

Alpha occ. eigenvalues -- -10.17175 -10.17175 -10.17071 -10.17071 -10.17062

Alpha occ. eigenvalues -- -10.17062 -10.17017 -10.17017 -10.17017 -10.17017

Alpha occ. eigenvalues -- -10.16913 -10.16913 -10.15957 -10.15957 -10.15956

Alpha occ. eigenvalues -- -10.15956 -10.15952 -10.15952 -10.15943 -10.15943

Alpha occ. eigenvalues -- -10.15921 -10.15921 -10.15873 -10.15873 -10.15799

Alpha occ. eigenvalues -- -10.15799 -10.15793 -10.15793 -10.15777 - 10.15777

Alpha occ. eigenvalues -- -10.15733 -10.15733 -10.15698 -10.15698 -10.15646

Alpha occ. eigenvalues -- -10.15646 -1.14006 -1.13899-0.94819 -0.94817

Alpha occ. eigenvalues -- -0.89377 -0.88570 -0.88189-0.87702 -0.87196

Alpha occ. eigenvalues -- $-0.86840-0.86840-0.86437-0.86435-0.86121$

Alpha occ. eigenvalues -- -0.85943 -0.85579 -0.85117-0.82542 -0.82540

Alpha occ. eigenvalues -- -0.82311 -0.82183 -0.82182 -0.82010-0.81996

Alpha occ. eigenvalues -- -0.81438 -0.80278 -0.79993-0.78813-0.78084

Alpha occ. eigenvalues -- -0.78074 -0.77416 -0.77413-0.76822 -0.75185

Alpha occ. eigenvalues -- $-0.74778-0.74746-0.74737-0.74700-0.74524$

Alpha occ. eigenvalues -- -0.74411 -0.74340 -0.74306 -0.74137 -0.73244

Alpha occ. eigenvalues -- -0.72490 -0.72455 -0.71958 -0.71933-0.71500

Alpha occ. eigenvalues -- -0.71192 -0.71120 -0.68843-0.68529 -0.68494

Alpha occ. eigenvalues -- $-0.68488-0.68387-0.68356-0.68348-0.68323$

Alpha occ. eigenvalues -- $-0.68320-0.68285-0.68283-0.68252-0.68246$

Alpha occ. eigenvalues -- $-0.68131-0.68131-0.68115-0.68115-0.66023$

Alpha occ. eigenvalues -- -0.64900 -0.64687 -0.64448 -0.63846 -0.63572

Alpha occ. eigenvalues -- -0.63236 -0.63078 -0.62671 -0.62336-0.62315

Alpha occ. eigenvalues -- -0.60953 -0.60753 -0.60725 -0.60701 -0.60339

Alpha occ. eigenvalues -- -0.60306 -0.60153 -0.59951 -0.59513-0.58573

Alpha occ. eigenvalues -- $-0.58420-0.58349-0.58271-0.57560-0.57332$

Alpha occ. eigenvalues -- $-0.56983-0.55729-0.55443-0.55430-0.55355$

Alpha occ. eigenvalues -- $-0.55350-0.55261-0.55105-0.55097-0.55019$

Alpha occ. eigenvalues -- $-0.55009-0.54152-0.53553-0.52827-0.51448$

Alpha occ. eigenvalues -- $-0.50248-0.50134-0.50133-0.49814-0.49724$

Alpha occ. eigenvalues -- -0.49701 -0.49652 -0.49615 -0.49226 -0.48962

Alpha occ. eigenvalues -- -0.48935 -0.48603-0.48481 -0.48368 -0.48278

Alpha occ. eigenvalues -- $-0.47886-0.47232-0.47081-0.47048-0.46863$

Alpha occ. eigenvalues -- -0.46715 -0.46712 -0.46521 -0.45603-0.45577

Alpha occ. eigenvalues -- -0.45530 -0.45405 -0.45287 -0.45238 -0.45125 
Alpha occ. eigenvalues -- -0.45113 -0.45046 -0.45017 -0.44936 -0.44673 Alpha occ. eigenvalues -- $-0.44634-0.44628-0.44619-0.44588-0.44515$ Alpha occ. eigenvalues -- $-0.44506-0.44503-0.44482-0.44352-0.44336$ Alpha occ. eigenvalues -- $-0.44309-0.44309-0.44207-0.44188-0.44055$ Alpha occ. eigenvalues -- $-0.43981-0.43058-0.43004-0.42988-0.42986$ Alpha occ. eigenvalues -- $-0.42778-0.42721-0.42626-0.42625-0.42384$ Alpha occ. eigenvalues -- $-0.42369-0.42360-0.42351-0.42093-0.42089$ Alpha occ. eigenvalues -- $-0.42011-0.42001-0.41123-0.40292-0.40181$ Alpha occ. eigenvalues -- -0.40115 -0.40052 -0.40016 -0.39809-0.39791 Alpha occ. eigenvalues -- $-0.39766-0.39760-0.39732-0.39725-0.39673$ Alpha occ. eigenvalues -- $-0.39655-0.39462-0.39407-0.39323-0.39309$ Alpha occ. eigenvalues -- $-0.38984-0.38813-0.38713-0.38627-0.38126$ Alpha occ. eigenvalues -- $-0.38118-0.38078-0.38044-0.37867-0.37835$ Alpha occ. eigenvalues -- -0.37724 -0.37722 -0.37489-0.37448 -0.37406 Alpha occ. eigenvalues -- $-0.37103-0.37080-0.37008-0.36942-0.36918$ Alpha occ. eigenvalues -- $-0.36875-0.36850-0.36786-0.36780-0.36619$ Alpha occ. eigenvalues -- $-0.36607-0.36570-0.36556-0.36411-0.36399$ Alpha occ. eigenvalues -- $-0.36361-0.36121-0.36062-0.35968-0.35695$ Alpha occ. eigenvalues -- -0.35629 -0.35250 -0.35239 -0.35199 -0.35183 Alpha occ. eigenvalues -- $-0.35162-0.34983-0.34769-0.34709-0.34687$ Alpha occ. eigenvalues -- $-0.34328-0.34077-0.33911-0.33888-0.33837$ Alpha occ. eigenvalues -- $-0.33402-0.33356-0.33285-0.33229-0.33157$ Alpha occ. eigenvalues -- $-0.33071-0.33065-0.32840-0.32835-0.32806$ Alpha occ. eigenvalues -- $-0.32803-0.32502-0.32480-0.32257-0.32236$ Alpha occ. eigenvalues -- $-0.32185-0.32182-0.31986-0.31978-0.31901$ Alpha occ. eigenvalues -- $-0.31900-0.30198-0.29608-0.29122-0.29090$ Alpha occ. eigenvalues -- $-0.29084-0.28955-0.28231-0.28167-0.28008$ Alpha occ. eigenvalues -- $-0.26576-0.26048-0.26036-0.26029-0.26020$ Alpha occ. eigenvalues -- $-0.25719-0.25711-0.25608-0.25600-0.24788$ Alpha occ. eigenvalues -- $-0.24143-0.23784-0.23254-0.22924-0.22902$ Alpha occ. eigenvalues -- $-0.22864-0.22257-0.22228-0.21433$ Alpha virt. eigenvalues -- $-0.08551-0.05522-0.04688-0.04579-0.04563$ Alpha virt. eigenvalues -- $-0.03725-0.03700-0.02652-0.02038-0.01508$ Alpha virt. eigenvalues -- $-0.01180-0.01143-0.01140-0.01094-0.01035$ Alpha virt. eigenvalues -- $-0.00848-0.00804-0.00729-0.006780 .01198$ Alpha virt. eigenvalues -- 0.012240 .028230 .028430 .032630 .03265 Alpha virt. eigenvalues -- 0.037630 .046850 .046860 .047530 .04764 Alpha virt. eigenvalues -- 0.051630 .060790 .060960 .061300 .06155 Alpha virt. eigenvalues -- 0.062950 .075080 .078760 .080550 .08159 Alpha virt. eigenvalues -- 0.082440 .083810 .085560 .086580 .08703 Alpha virt. eigenvalues -- 0.088490 .091140 .091390 .092960 .09304 Alpha virt. eigenvalues -- 0.093950 .094650 .095390 .096330 .09717 Alpha virt. eigenvalues -- 0.097400 .099660 .100300 .101460 .10166 Alpha virt. eigenvalues -- 0.104660 .104950 .106300 .106440 .10809 Alpha virt. eigenvalues -- 0.108100 .109450 .110070 .111350 .11179 Alpha virt. eigenvalues -- 0.112480 .113210 .114990 .115930 .11639 Alpha virt. eigenvalues -- 0.119050 .119400 .120960 .121050 .12147 Alpha virt. eigenvalues -- 0.122460 .122680 .123520 .123710 .12618 Alpha virt. eigenvalues -- 0.126210 .127690 .128740 .130120 .13081 Alpha virt. eigenvalues -- 0.132060 .134020 .134310 .136610 .13825 Alpha virt. eigenvalues -- 0.138360 .139640 .140070 .140890 .14352 Alpha virt. eigenvalues -- 0.144330 .146870 .150060 .153350 .15430 Alpha virt. eigenvalues -- 0.155200 .157010 .157070 .157820 .15888 Alpha virt. eigenvalues -- 0.161430 .161600 .161930 .162050 .16236 Alpha virt. eigenvalues -- 0.162590 .164920 .167680 .169830 .16994 Alpha virt. eigenvalues -- 0.171130 .171410 .172730 .173530 .17434 
Alpha virt. eigenvalues -- 0.174960 .176100 .176250 .176600 .17750 Alpha virt. eigenvalues -- 0.178210 .179180 .180720 .181820 .18231 Alpha virt. eigenvalues -- 0.183340 .183430 .183840 .184100 .18572 Alpha virt. eigenvalues -- 0.186050 .186160 .187200 .188350 .18974 Alpha virt. eigenvalues -- 0.190530 .192050 .192600 .195150 .19550 Alpha virt. eigenvalues -- 0.195810 .199460 .200250 .201890 .20258 Alpha virt. eigenvalues -- 0.204290 .204430 .208120 .209480 .20971 Alpha virt. eigenvalues -- 0.210080 .210460 .212550 .212950 .21769 Alpha virt. eigenvalues -- 0.217750 .221240 .222130 .222480 .22510 Alpha virt. eigenvalues -- 0.225480 .226520 .227740 .228180 .22889 Alpha virt. eigenvalues -- 0.233550 .234370 .235710 .236390 .24123 Alpha virt. eigenvalues -- 0.241380 .241510 .242490 .243210 .24494 Alpha virt. eigenvalues -- 0.246180 .246700 .247470 .248540 .24889 Alpha virt. eigenvalues -- 0.249450 .249850 .250560 .259030 .26193 Alpha virt. eigenvalues -- 0.263100 .263200 .264070 .268840 .27050 Alpha virt. eigenvalues -- 0.271350 .271610 .272520 .274110 .27529 Alpha virt. eigenvalues -- 0.275490 .277620 .279470 .279840 .28039 Alpha virt. eigenvalues -- 0.282570 .284110 .285070 .286740 .29053 Alpha virt. eigenvalues -- 0.290940 .293010 .297290 .299230 .29957 Alpha virt. eigenvalues -- 0.300790 .301310 .301980 .303260 .30464 Alpha virt. eigenvalues -- 0.309210 .310770 .312280 .313330 .31528 Alpha virt. eigenvalues -- 0.315900 .316610 .316920 .319450 .32045 Alpha virt. eigenvalues -- 0.324630 .324640 .325000 .327470 .32863 Alpha virt. eigenvalues -- 0.330560 .332680 .333990 .334990 .33693 Alpha virt. eigenvalues -- 0.337540 .338920 .340310 .341460 .34218 Alpha virt. eigenvalues -- 0.345530 .346000 .346910 .349280 .35202 Alpha virt. eigenvalues -- 0.354000 .356890 .359830 .363280 .36380 Alpha virt. eigenvalues -- 0.364140 .366630 .368890 .371130 .37394 Alpha virt. eigenvalues -- 0.375060 .376820 .377240 .378050 .38458 Alpha virt. eigenvalues -- 0.384690 .386580 .390240 .392630 .39549 Alpha virt. eigenvalues -- 0.395520 .395910 .399170 .401140 .40199 Alpha virt. eigenvalues -- 0.403760 .406380 .408650 .410910 .41153 Alpha virt. eigenvalues -- 0.414200 .414490 .416460 .416760 .41748 Alpha virt. eigenvalues -- 0.421740 .424920 .425000 .431010 .43113 Alpha virt. eigenvalues -- 0.431240 .432220 .438120 .438540 .43892 Alpha virt. eigenvalues -- 0.439250 .440580 .440880 .441230 .44126 Alpha virt. eigenvalues -- 0.442820 .444260 .445140 .446530 .44773 Alpha virt. eigenvalues -- 0.448240 .448250 .448710 .449240 .45134 Alpha virt. eigenvalues -- 0.451600 .452630 .454440 .454620 .45782 Alpha virt. eigenvalues -- 0.459080 .460800 .461230 .462740 .46503 Alpha virt. eigenvalues -- 0.465650 .467430 .467940 .470230 .47034 Alpha virt. eigenvalues -- 0.470470 .470700 .471960 .472440 .47497 Alpha virt. eigenvalues -- 0.475650 .476600 .481030 .481080 .48189 Alpha virt. eigenvalues -- 0.482440 .484710 .485170 .485840 .48775 Alpha virt. eigenvalues -- 0.488290 .488790 .489590 .491350 .49292 Alpha virt. eigenvalues -- 0.494000 .494390 .495470 .496210 .49777 Alpha virt. eigenvalues -- 0.498220 .498490 .499860 .500420 .50355 Alpha virt. eigenvalues -- 0.505960 .507850 .508700 .509550 .51077 Alpha virt. eigenvalues -- 0.511290 .512360 .512380 .513800 .51785 Alpha virt. eigenvalues -- 0.518510 .519900 .520070 .523250 .52406 Alpha virt. eigenvalues -- 0.525790 .530130 .532410 .534930 .53493 Alpha virt. eigenvalues -- 0.541970 .548280 .549420 .550130 .55410 Alpha virt. eigenvalues -- 0.554870 .557670 .558440 .560050 .56075 Alpha virt. eigenvalues -- 0.563260 .563340 .565180 .566910 .56731 Alpha virt. eigenvalues -- 0.570150 .571980 .571990 .573530 .57556 Alpha virt. eigenvalues -- 0.577130 .578800 .579510 .579930 .58211 
Alpha virt. eigenvalues -- 0.582320 .584610 .587390 .589030 .59027 Alpha virt. eigenvalues -- 0.591040 .593410 .593770 .595870 .59593 Alpha virt. eigenvalues -- 0.599900 .600320 .600670 .602040 .60428 Alpha virt. eigenvalues -- 0.608060 .608470 .609090 .610100 .61417 Alpha virt. eigenvalues -- 0.614390 .618610 .618650 .619490 .61970 Alpha virt. eigenvalues -- 0.620720 .621050 .621410 .621870 .62235 Alpha virt. eigenvalues -- 0.622960 .623690 .624220 .624490 .62545 Alpha virt. eigenvalues -- 0.625620 .628390 .630980 .633380 .63347 Alpha virt. eigenvalues -- 0.635600 .636260 .640780 .641920 .64391 Alpha virt. eigenvalues -- 0.645450 .646030 .647840 .648410 .64908 Alpha virt. eigenvalues -- 0.649940 .650200 .650700 .652030 .65468 Alpha virt. eigenvalues -- 0.656230 .659160 .665760 .666450 .66839 Alpha virt. eigenvalues -- 0.668730 .670780 .671330 .672390 .67286 Alpha virt. eigenvalues -- 0.672960 .673420 .674660 .674690 .67494 Alpha virt. eigenvalues -- 0.675300 .675390 .676270 .677510 .67801 Alpha virt. eigenvalues -- 0.678010 .679190 .679560 .680370 .68048 Alpha virt. eigenvalues -- 0.680780 .681740 .682110 .682780 .68321 Alpha virt. eigenvalues -- 0.683790 .684000 .685110 .686400 .68714 Alpha virt. eigenvalues -- 0.687370 .688170 .688870 .689620 .68996 Alpha virt. eigenvalues -- 0.690430 .690530 .691130 .691220 .69199 Alpha virt. eigenvalues -- 0.692240 .692760 .693950 .695060 .69543 Alpha virt. eigenvalues -- 0.695720 .695970 .697450 .698790 .70028 Alpha virt. eigenvalues -- 0.700450 .701980 .703150 .704770 .70568 Alpha virt. eigenvalues -- 0.706240 .707050 .707160 .708130 .70959 Alpha virt. eigenvalues -- 0.710230 .711480 .711920 .712220 .71248 Alpha virt. eigenvalues -- 0.713300 .713650 .713960 .714780 .71620 Alpha virt. eigenvalues -- 0.716360 .716810 .718540 .719820 .71987 Alpha virt. eigenvalues -- 0.720000 .720210 .721990 .723210 .72414 Alpha virt. eigenvalues -- 0.724790 .726060 .726450 .727300 .72754 Alpha virt. eigenvalues -- 0.727740 .728280 .729390 .729700 .73164 Alpha virt. eigenvalues -- 0.732180 .732900 .733630 .734300 .73448 Alpha virt. eigenvalues -- 0.734530 .735740 .735960 .737310 .73878 Alpha virt. eigenvalues -- 0.738820 .739430 .740100 .741080 .74328 Alpha virt. eigenvalues -- 0.743730 .743960 .744700 .745740 .74597 Alpha virt. eigenvalues -- 0.747240 .747760 .748640 .748930 .75070 Alpha virt. eigenvalues -- 0.750970 .751660 .753280 .753400 .75361 Alpha virt. eigenvalues -- 0.754460 .756400 .757410 .758250 .75992 Alpha virt. eigenvalues -- 0.760180 .760890 .761310 .761360 .76277 Alpha virt. eigenvalues -- 0.763090 .763340 .765030 .765930 .76665 Alpha virt. eigenvalues -- 0.767680 .768340 .768560 .770640 .77282 Alpha virt. eigenvalues -- 0.774260 .775730 .777460 .778280 .77883 Alpha virt. eigenvalues -- 0.780310 .783850 .784180 .785160 .78614 Alpha virt. eigenvalues -- 0.787590 .788340 .790190 .791230 .79239 Alpha virt. eigenvalues -- 0.794890 .796700 .796970 .798690 .79970 Alpha virt. eigenvalues -- 0.800990 .801230 .801820 .802670 .80383 Alpha virt. eigenvalues -- 0.804190 .804320 .808570 .812030 .81337 Alpha virt. eigenvalues -- 0.816510 .816970 .817600 .819870 .81989 Alpha virt. eigenvalues -- 0.820810 .823430 .824250 .825810 .82733 Alpha virt. eigenvalues -- 0.828210 .828660 .830610 .833150 .83375 Alpha virt. eigenvalues -- 0.834160 .836950 .839580 .841190 .84268 Alpha virt. eigenvalues -- 0.843160 .844650 .845170 .845190 .84617 Alpha virt. eigenvalues -- 0.848020 .848470 .849990 .850420 .85138 Alpha virt. eigenvalues -- 0.851870 .852360 .852430 .853000 .85482 Alpha virt. eigenvalues -- 0.858330 .859080 .860450 .863880 .86617 Alpha virt. eigenvalues -- 0.866260 .869350 .870420 .871280 .87313 Alpha virt. eigenvalues -- 0.873740 .874330 .878820 .880270 .88187 
Alpha virt. eigenvalues -- 0.882220 .882990 .884610 .888850 .88926 Alpha virt. eigenvalues -- 0.891010 .894650 .897670 .898430 .90056 Alpha virt. eigenvalues -- 0.902050 .904190 .908300 .909560 .91229 Alpha virt. eigenvalues -- 0.915970 .916130 .918350 .925590 .93442 Alpha virt. eigenvalues -- 0.936190 .941360 .943870 .953550 .95511 Alpha virt. eigenvalues -- 0.958480 .961860 .964780 .968470 .97046 Alpha virt. eigenvalues -- 0.981770 .985300 .990970 .996640 .99999 Alpha virt. eigenvalues -- 1.000261 .005581 .011141 .014521 .02199 Alpha virt. eigenvalues -- 1.022191 .023281 .025021 .028551 .03332 Alpha virt. eigenvalues -- 1.034451 .038711 .039211 .041461 .04359 Alpha virt. eigenvalues -- 1.047081 .047391 .047891 .053181 .05583 Alpha virt. eigenvalues -- 1.057011 .061731 .070681 .071911 .07521 Alpha virt. eigenvalues -- 1.080961 .083141 .084121 .088711 .09103 Alpha virt. eigenvalues -- 1.094731 .095781 .098081 .098291 .09893 Alpha virt. eigenvalues -- 1.100041 .100901 .102251 .105851 .10600 Alpha virt. eigenvalues -- 1.109641 .110091 .110911 .113361 .11560 Alpha virt. eigenvalues -- 1.120481 .120961 .128011 .128681 .13230 Alpha virt. eigenvalues -- 1.132331 .132781 .133091 .137251 .13860 Alpha virt. eigenvalues -- 1.139291 .139901 .140711 .141531 .14468 Alpha virt. eigenvalues -- 1.146351 .146411 .147361 .149071 .14916 Alpha virt. eigenvalues -- 1.155341 .157501 .163201 .171061 .17136 Alpha virt. eigenvalues -- 1.175001 .176581 .188541 .191071 .19278 Alpha virt. eigenvalues -- 1.194371 .197141 .201431 .202851 .20320 Alpha virt. eigenvalues -- 1.206411 .214211 .221501 .244381 .24789 Alpha virt. eigenvalues -- 1.254241 .255971 .260181 .262191 .26428 Alpha virt. eigenvalues -- 1.266701 .268041 .283891 .291221 .29405 Alpha virt. eigenvalues -- 1.297991 .303661 .305531 .313971 .31459 Alpha virt. eigenvalues -- 1.321171 .323321 .324091 .324191 .32452 Alpha virt. eigenvalues -- 1.334651 .335161 .344571 .349201 .35737 Alpha virt. eigenvalues -- 1.357401 .359611 .360441 .364151 .36890 Alpha virt. eigenvalues -- 1.370981 .383401 .385351 .409551 .40966 Alpha virt. eigenvalues -- 1.419981 .422881 .432991 .437011 .44812 Alpha virt. eigenvalues -- 1.455831 .460821 .471721 .475571 .51097 Alpha virt. eigenvalues -- 1.560181 .564491 .576331 .583681 .58789 Alpha virt. eigenvalues -- 1.588411 .588491 .591391 .591601 .59374 Alpha virt. eigenvalues -- 1.594441 .601491 .612661 .737621 .81173 Alpha virt. eigenvalues -- 1.830511 .89367

\section{Chloroform}

Alpha occ. eigenvalues -- -19.21933 -19.21924 -14.34333 -14.34332 -10.29585 Alpha occ. eigenvalues -- -10.29582 -10.26299-10.26289-10.23773 -10.23762 Alpha occ. eigenvalues -- -10.22237 -10.22220 -10.21361 -10.21354-10.20515 Alpha occ. eigenvalues -- -10.20497 -10.20473 -10.20462 -10.20434-10.20428 Alpha occ. eigenvalues -- -10.19762 -10.19734 -10.19703 -10.19702 -10.19690 Alpha occ. eigenvalues -- -10.19689 -10.19681 -10.19648 -10.19646 -10.19637 Alpha occ. eigenvalues -- -10.19622 -10.19538 -10.19495 -10.19494 -10.19479 Alpha occ. eigenvalues -- -10.19479 -10.19467 -10.19467-10.19351-10.19329 Alpha occ. eigenvalues -- -10.19265 -10.19246 -10.19181 -10.19169-10.19155 Alpha occ. eigenvalues -- -10.19079 -10.19022 -10.19014 -10.19011 -10.19001 Alpha occ. eigenvalues -- -10.18878 -10.18864 -10.18803-10.18724 -10.18493 Alpha occ. eigenvalues -- -10.18468 -10.18380 -10.18352 -10.18316 -10.18306 Alpha occ. eigenvalues -- -10.18305 -10.18267 -10.18265 -10.18258 -10.18252 Alpha occ. eigenvalues -- -10.18240 -10.18235 -10.18231 -10.18117-10.18091 Alpha occ. eigenvalues -- -10.18080 -10.18015 -10.17998 -10.17960 -10.17948 Alpha occ. eigenvalues -- -10.17942 -10.17942 -10.17929-10.17911 -10.17892 
Alpha occ. eigenvalues -- -10.17889 -10.17875 -10.17867 -10.17864-10.17843 Alpha occ. eigenvalues -- -10.17813 -10.17785 -10.17759 -10.17722 -10.17672 Alpha occ. eigenvalues -- -10.17643 -10.17600 - 10.15955 -10.15939 -10.15934 Alpha occ. eigenvalues -- -10.15931 -10.15926 -10.15920 -10.15903-10.15889 Alpha occ. eigenvalues -- -10.15885 -10.15878 -10.15875 -10.15859 -10.15841 Alpha occ. eigenvalues -- -10.15828 -10.15806 -10.15802 -10.15801 -10.15796 Alpha occ. eigenvalues -- -10.15787 -10.15785 -10.15764-10.15746-10.15730 Alpha occ. eigenvalues -- -10.15678 -1.15014 - 1.14907 -0.95855 -0.95849 Alpha occ. eigenvalues -- -0.90392 -0.89410 -0.89085 -0.88720 -0.88094 Alpha occ. eigenvalues -- -0.87410 -0.87404 -0.87186 -0.87141 -0.86714 Alpha occ. eigenvalues -- -0.86559 -0.86367 -0.85914 -0.82872 -0.82859 Alpha occ. eigenvalues -- $-0.82803-0.82681-0.82653-0.82605-0.82355$ Alpha occ. eigenvalues -- $-0.81890-0.80960-0.80732-0.79570-0.78678$ Alpha occ. eigenvalues -- -0.78668 -0.78228 -0.78192 -0.77757 -0.75981 Alpha occ. eigenvalues -- $-0.75405-0.75337-0.75290-0.75278-0.75212$ Alpha occ. eigenvalues -- $-0.75130-0.75076-0.75003-0.74884-0.73815$ Alpha occ. eigenvalues -- -0.73213 -0.73166 -0.72614 -0.72593 -0.72336 Alpha occ. eigenvalues -- -0.72043 -0.71970 -0.69661 -0.68511 -0.68504 Alpha occ. eigenvalues -- -0.68497 -0.68460 -0.68442 -0.68433-0.68418 Alpha occ. eigenvalues -- $-0.68363-0.68288-0.68285-0.68281-0.68274$ Alpha occ. eigenvalues -- -0.68250 -0.68248 -0.68232 -0.68141 -0.66911 Alpha occ. eigenvalues -- -0.65915 -0.65516 -0.65125 -0.64587 -0.64414 Alpha occ. eigenvalues -- -0.63918 -0.63898 -0.63490 -0.63027 -0.62912 Alpha occ. eigenvalues -- -0.61686 -0.61339-0.61214-0.61163-0.60997 Alpha occ. eigenvalues -- -0.60934 -0.60805 -0.60659 -0.60162 -0.59316 Alpha occ. eigenvalues -- $-0.59110-0.59011-0.58889-0.58365-0.58165$ Alpha occ. eigenvalues -- $-0.57760-0.56703-0.56146-0.55804-0.55715$ Alpha occ. eigenvalues -- -0.55671 -0.55631 -0.55544-0.55528 -0.55472 Alpha occ. eigenvalues -- $-0.55409-0.54752-0.54309-0.53662-0.52196$ Alpha occ. eigenvalues -- $-0.51113-0.50680-0.50530-0.50519-0.50332$ Alpha occ. eigenvalues -- -0.50280 -0.50234 -0.50203-0.50000 -0.49801 Alpha occ. eigenvalues -- -0.49634 -0.49392 -0.49279 -0.49101 -0.49018 Alpha occ. eigenvalues -- $-0.48439-0.47946-0.47548-0.47456-0.47425$ Alpha occ. eigenvalues -- -0.47333 -0.47266 -0.47219-0.46223 -0.46078 Alpha occ. eigenvalues -- -0.46004 -0.45958 -0.45870 -0.45815 -0.45744 Alpha occ. eigenvalues -- -0.45594 -0.45523 -0.45464 -0.45413 -0.45400 Alpha occ. eigenvalues -- -0.45232 -0.45164 -0.45097 -0.45070 -0.44975 Alpha occ. eigenvalues -- -0.44881 -0.44754 -0.44704-0.44545 -0.44535 Alpha occ. eigenvalues -- $-0.44529-0.44513-0.44473-0.44461-0.44445$ Alpha occ. eigenvalues -- -0.44377 -0.43590 -0.43565 -0.43393-0.43305 Alpha occ. eigenvalues -- $-0.43135-0.43080-0.43042-0.42977-0.42689$ Alpha occ. eigenvalues -- -0.42673 -0.42549-0.42529 -0.42493-0.42459 Alpha occ. eigenvalues -- -0.42407 -0.42328 -0.41998 -0.41282 -0.40890 Alpha occ. eigenvalues -- -0.40757 -0.40503 -0.40407 -0.40311 -0.40252 Alpha occ. eigenvalues -- -0.40120 -0.40076 -0.40073-0.40033-0.40002 Alpha occ. eigenvalues -- -0.39965 -0.39924 -0.39898 -0.39861 -0.39768 Alpha occ. eigenvalues -- -0.39718 -0.39647 -0.39523-0.39438 -0.38331 Alpha occ. eigenvalues -- -0.38291 -0.38185 -0.38138 -0.38054-0.38019 Alpha occ. eigenvalues -- -0.37998 -0.37976 -0.37939-0.37852 -0.37739 Alpha occ. eigenvalues -- $-0.37692-0.37611-0.37574-0.37550-0.37475$ Alpha occ. eigenvalues -- $-0.37183-0.36986-0.36934-0.36885-0.36818$ Alpha occ. eigenvalues -- $-0.36810-0.36800-0.36753-0.36733-0.36708$ Alpha occ. eigenvalues -- -0.36689 -0.36635 -0.36615 -0.36541 -0.36422 Alpha occ. eigenvalues -- -0.36342 -0.35982 -0.35943-0.35729 -0.35716 Alpha occ. eigenvalues -- -0.35567 -0.35549 -0.35315 -0.35268 -0.35207 Alpha occ. eigenvalues -- -0.35063 -0.34659-0.33874 -0.33873-0.33805 
Alpha occ. eigenvalues -- -0.33774 -0.33758 -0.33614 -0.33556 -0.33534 Alpha occ. eigenvalues -- $-0.33232-0.33221-0.33165-0.33153-0.33129$ Alpha occ. eigenvalues -- $-0.33081-0.32993-0.32951-0.32384-0.32380$ Alpha occ. eigenvalues -- $-0.32349-0.32338-0.32265-0.32245-0.32226$ Alpha occ. eigenvalues -- $-0.32143-0.31042-0.30905-0.30428-0.29910$ Alpha occ. eigenvalues -- $-0.29795-0.29721-0.29354-0.29252-0.28941$ Alpha occ. eigenvalues -- $-0.27554-0.26730-0.26724-0.26708-0.26693$ Alpha occ. eigenvalues -- $-0.26572-0.26561-0.26483-0.26402-0.25718$ Alpha occ. eigenvalues -- $-0.25146-0.24560-0.24139-0.23785-0.23689$ Alpha occ. eigenvalues -- $-0.23632-0.23176-0.23148-0.22565$

Alpha virt. eigenvalues -- $-0.09475-0.06481-0.05607-0.05367-0.05351$

Alpha virt. eigenvalues -- $-0.04895-0.04809-0.03843-0.03027-0.02352$

Alpha virt. eigenvalues -- $-0.02169-0.01829-0.01812-0.01760-0.01716$ Alpha virt. eigenvalues -- -0.01670 -0.01623 -0.01547 -0.01449 0.00219 Alpha virt. eigenvalues -- 0.002690 .020390 .022330 .024300 .02450 Alpha virt. eigenvalues -- 0.027680 .039610 .040170 .042100 .04456 Alpha virt. eigenvalues -- 0.049840 .052850 .059350 .061960 .06201 Alpha virt. eigenvalues -- 0.062350 .064020 .065360 .068270 .07167 Alpha virt. eigenvalues -- 0.074600 .078870 .080680 .082340 .08361 Alpha virt. eigenvalues -- 0.086140 .087860 .088940 .091460 .09271 Alpha virt. eigenvalues -- 0.095130 .095500 .096010 .098470 .10119 Alpha virt. eigenvalues -- 0.104190 .104810 .105040 .105240 .10574 Alpha virt. eigenvalues -- 0.105860 .106280 .106960 .107130 .10787 Alpha virt. eigenvalues -- 0.108490 .110320 .111100 .112850 .11322 Alpha virt. eigenvalues -- 0.113250 .113570 .115050 .115500 .11596 Alpha virt. eigenvalues -- 0.116920 .118580 .119380 .120540 .12076 Alpha virt. eigenvalues -- 0.122010 .123370 .124230 .124790 .12604 Alpha virt. eigenvalues -- 0.126490 .126920 .127790 .128970 .12988 Alpha virt. eigenvalues -- 0.131250 .132800 .133200 .133710 .13457 Alpha virt. eigenvalues -- 0.135920 .138130 .138530 .141080 .14277 Alpha virt. eigenvalues -- 0.145420 .147200 .150030 .151380 .15196 Alpha virt. eigenvalues -- 0.152760 .153940 .156480 .157130 .15875 Alpha virt. eigenvalues -- 0.160330 .161140 .161540 .162340 .16318 Alpha virt. eigenvalues -- 0.163440 .164560 .167550 .168310 .16883 Alpha virt. eigenvalues -- 0.170390 .171180 .173160 .173690 .17381 Alpha virt. eigenvalues -- 0.174380 .174790 .175770 .175870 .17647 Alpha virt. eigenvalues -- 0.176940 .177160 .177730 .178000 .17917 Alpha virt. eigenvalues -- 0.180110 .182430 .182720 .183220 .18515 Alpha virt. eigenvalues -- 0.186010 .186820 .187410 .188510 .18887 Alpha virt. eigenvalues -- 0.189640 .190620 .192160 .193180 .19567 Alpha virt. eigenvalues -- 0.196150 .198140 .198480 .198780 .19940 Alpha virt. eigenvalues -- 0.200960 .202150 .203600 .204670 .20518 Alpha virt. eigenvalues -- 0.206400 .207390 .209280 .209750 .21241 Alpha virt. eigenvalues -- 0.214190 .216400 .217270 .218000 .22158 Alpha virt. eigenvalues -- 0.222880 .225030 .228410 .229970 .23075 Alpha virt. eigenvalues -- 0.232930 .233860 .235160 .237360 .23744 Alpha virt. eigenvalues -- 0.238790 .239980 .240640 .241200 .24261 Alpha virt. eigenvalues -- 0.243370 .245780 .246410 .248540 .25014 Alpha virt. eigenvalues -- 0.250680 .250920 .252410 .254050 .25453 Alpha virt. eigenvalues -- 0.255780 .256660 .258230 .261080 .26175 Alpha virt. eigenvalues -- 0.264420 .265290 .266570 .268070 .26863 Alpha virt. eigenvalues -- 0.270050 .271230 .272830 .272970 .27420 Alpha virt. eigenvalues -- 0.276730 .278610 .280580 .281070 .28269 Alpha virt. eigenvalues -- 0.287090 .287490 .290930 .293150 .29415 Alpha virt. eigenvalues -- 0.294710 .296970 .298220 .299680 .30033 Alpha virt. eigenvalues -- 0.302190 .303380 .305770 .306360 .30726 
Alpha virt. eigenvalues -- 0.310000 .312030 .312250 .314120 .31465 Alpha virt. eigenvalues -- 0.317330 .320790 .321490 .322320 .32421 Alpha virt. eigenvalues -- 0.325870 .326860 .327020 .327740 .32876 Alpha virt. eigenvalues -- 0.330150 .332120 .332800 .334380 .33514 Alpha virt. eigenvalues -- 0.336050 .337070 .338950 .342560 .34371 Alpha virt. eigenvalues -- 0.344510 .348440 .353000 .354220 .35756 Alpha virt. eigenvalues -- 0.357780 .360170 .360700 .364580 .36513 Alpha virt. eigenvalues -- 0.367640 .370370 .370610 .373460 .37474 Alpha virt. eigenvalues -- 0.376210 .380970 .381710 .382270 .38514 Alpha virt. eigenvalues -- 0.386220 .388400 .393370 .393650 .39372 Alpha virt. eigenvalues -- 0.396830 .400250 .400470 .405470 .40667 Alpha virt. eigenvalues -- 0.411300 .411860 .415210 .416920 .41775 Alpha virt. eigenvalues -- 0.419750 .421410 .424250 .426130 .42686 Alpha virt. eigenvalues -- 0.427990 .429110 .429660 .430300 .43223 Alpha virt. eigenvalues -- 0.433420 .434110 .435360 .436790 .43785 Alpha virt. eigenvalues -- 0.438740 .438910 .439910 .439970 .44076 Alpha virt. eigenvalues -- 0.442520 .443410 .444070 .445030 .44633 Alpha virt. eigenvalues -- 0.447070 .448510 .449790 .450270 .45083 Alpha virt. eigenvalues -- 0.453060 .453970 .454850 .456550 .45856 Alpha virt. eigenvalues -- 0.459850 .461090 .461600 .462660 .46327 Alpha virt. eigenvalues -- 0.464270 .464970 .465560 .465960 .46718 Alpha virt. eigenvalues -- 0.468670 .469760 .470370 .471430 .47198 Alpha virt. eigenvalues -- 0.474170 .474920 .476770 .478660 .47933 Alpha virt. eigenvalues -- 0.479830 .482190 .482730 .482980 .48401 Alpha virt. eigenvalues -- 0.487310 .487660 .490370 .492670 .49304 Alpha virt. eigenvalues -- 0.493570 .494090 .495640 .496760 .49809 Alpha virt. eigenvalues -- 0.499340 .500400 .501160 .502030 .50350 Alpha virt. eigenvalues -- 0.504450 .505000 .506440 .509160 .51116 Alpha virt. eigenvalues -- 0.511910 .514770 .517900 .521750 .52444 Alpha virt. eigenvalues -- 0.524720 .526190 .529960 .532170 .53325 Alpha virt. eigenvalues -- 0.533670 .539260 .545180 .547360 .54931 Alpha virt. eigenvalues -- 0.550620 .551150 .552370 .553900 .55539 Alpha virt. eigenvalues -- 0.556880 .559280 .559680 .561010 .56183 Alpha virt. eigenvalues -- 0.563220 .564610 .566470 .567700 .56988 Alpha virt. eigenvalues -- 0.571100 .571850 .573330 .574210 .57717 Alpha virt. eigenvalues -- 0.577970 .580630 .581840 .582760 .58409 Alpha virt. eigenvalues -- 0.585310 .586810 .587380 .590240 .59106 Alpha virt. eigenvalues -- 0.591260 .593680 .595160 .597240 .59909 Alpha virt. eigenvalues -- 0.599910 .600560 .603360 .604950 .60707 Alpha virt. eigenvalues -- 0.607360 .608420 .609900 .614010 .61620 Alpha virt. eigenvalues -- 0.617120 .618740 .619410 .620510 .62104 Alpha virt. eigenvalues -- 0.621250 .621580 .622700 .623030 .62399 Alpha virt. eigenvalues -- 0.625280 .626520 .627700 .629740 .63014 Alpha virt. eigenvalues -- 0.630900 .632290 .634020 .635450 .63643 Alpha virt. eigenvalues -- 0.638890 .639740 .641180 .642360 .64336 Alpha virt. eigenvalues -- 0.644200 .647080 .648540 .649480 .65187 Alpha virt. eigenvalues -- 0.652570 .656330 .657980 .659850 .66117 Alpha virt. eigenvalues -- 0.662290 .663480 .664750 .665110 .66677 Alpha virt. eigenvalues -- 0.668150 .668520 .669450 .670050 .67060 Alpha virt. eigenvalues -- 0.671200 .672100 .672340 .673070 .67356 Alpha virt. eigenvalues -- 0.674140 .674660 .674880 .675770 .67634 Alpha virt. eigenvalues -- 0.676670 .676990 .678250 .679420 .68035 Alpha virt. eigenvalues -- 0.680750 .682260 .683450 .683600 .68486 Alpha virt. eigenvalues -- 0.685810 .686090 .686740 .687320 .68808 Alpha virt. eigenvalues -- 0.689050 .689080 .690360 .690710 .69128 Alpha virt. eigenvalues -- 0.691760 .692770 .692920 .693640 .69494 
Alpha virt. eigenvalues -- 0.695200 .695880 .696230 .697750 .69807 Alpha virt. eigenvalues -- 0.698260 .699260 .700120 .700480 .70088 Alpha virt. eigenvalues -- 0.701760 .702190 .702840 .703120 .70333 Alpha virt. eigenvalues -- 0.704670 .705180 .706660 .706920 .70815 Alpha virt. eigenvalues -- 0.708240 .708260 .709970 .711440 .71239 Alpha virt. eigenvalues -- 0.713120 .714340 .715190 .716490 .71713 Alpha virt. eigenvalues -- 0.718640 .719360 .719980 .721300 .72188 Alpha virt. eigenvalues -- 0.722300 .723690 .724450 .724660 .72512 Alpha virt. eigenvalues -- 0.726300 .726820 .727790 .728120 .72830 Alpha virt. eigenvalues -- 0.728720 .728820 .730470 .730930 .73255 Alpha virt. eigenvalues -- 0.732980 .733530 .734080 .734490 .73557 Alpha virt. eigenvalues -- 0.736830 .737270 .738250 .739260 .74032 Alpha virt. eigenvalues -- 0.741340 .742360 .743500 .743890 .74495 Alpha virt. eigenvalues -- 0.745640 .746150 .747090 .747720 .74960 Alpha virt. eigenvalues -- 0.749710 .750180 .750670 .752080 .75237 Alpha virt. eigenvalues -- 0.753380 .754950 .755330 .757310 .75819 Alpha virt. eigenvalues -- 0.759690 .760200 .761070 .761380 .76255 Alpha virt. eigenvalues -- 0.763530 .764100 .764480 .766120 .76722 Alpha virt. eigenvalues -- 0.767380 .768270 .769710 .770360 .77178 Alpha virt. eigenvalues -- 0.771970 .774780 .775760 .776290 .77734 Alpha virt. eigenvalues -- 0.778710 .779300 .781520 .783240 .78520 Alpha virt. eigenvalues -- 0.786950 .788330 .788480 .789750 .79201 Alpha virt. eigenvalues -- 0.793410 .794530 .795180 .796810 .79778 Alpha virt. eigenvalues -- 0.798450 .800150 .800740 .801650 .80232 Alpha virt. eigenvalues -- 0.803750 .805290 .806560 .808630 .80937 Alpha virt. eigenvalues -- 0.810570 .811480 .813830 .815840 .81607 Alpha virt. eigenvalues -- 0.816630 .818580 .819860 .820420 .82226 Alpha virt. eigenvalues -- 0.823190 .824930 .826430 .827380 .82957 Alpha virt. eigenvalues -- 0.830210 .832150 .835320 .836410 .83820 Alpha virt. eigenvalues -- 0.839280 .841090 .841870 .842200 .84310 Alpha virt. eigenvalues -- 0.843590 .844320 .845630 .848140 .84884 Alpha virt. eigenvalues -- 0.850600 .851180 .851680 .852820 .85433 Alpha virt. eigenvalues -- 0.856040 .858370 .858660 .860030 .86070 Alpha virt. eigenvalues -- 0.862360 .863460 .865700 .866560 .86829 Alpha virt. eigenvalues -- 0.869900 .873270 .875560 .879350 .88260 Alpha virt. eigenvalues -- 0.884440 .885680 .887310 .888090 .88894 Alpha virt. eigenvalues -- 0.891530 .896080 .897040 .900500 .90514 Alpha virt. eigenvalues -- 0.905600 .908170 .910170 .916620 .91873 Alpha virt. eigenvalues -- 0.920810 .926250 .927200 .929380 .93922 Alpha virt. eigenvalues -- 0.941120 .946910 .949120 .952080 .95887 Alpha virt. eigenvalues -- 0.959290 .961470 .967980 .976250 .97721 Alpha virt. eigenvalues -- 0.979510 .983560 .989050 .994630 .99848 Alpha virt. eigenvalues -- 1.000551 .004801 .007451 .010071 .01121 Alpha virt. eigenvalues -- 1.012581 .014841 .017201 .017521 .02249 Alpha virt. eigenvalues -- 1.026191 .027051 .029961 .033241 .03471 Alpha virt. eigenvalues -- 1.037551 .038651 .042541 .046341 .04874 Alpha virt. eigenvalues -- 1.051331 .052971 .054401 .059181 .06282 Alpha virt. eigenvalues -- 1.073111 .076161 .082871 .087961 .09087 Alpha virt. eigenvalues -- 1.092061 .094061 .097031 .097491 .09856 Alpha virt. eigenvalues -- 1.099841 .102241 .103571 .104721 .10581 Alpha virt. eigenvalues -- 1.106741 .108451 .109141 .111991 .11403 Alpha virt. eigenvalues -- 1.115991 .118611 .122581 .126011 .13035 Alpha virt. eigenvalues -- 1.131371 .132971 .135241 .137261 .13737 Alpha virt. eigenvalues -- 1.138351 .141151 .142041 .143731 .14459 Alpha virt. eigenvalues -- 1.146471 .147851 .148141 .151051 .15297 Alpha virt. eigenvalues -- 1.154241 .155641 .157771 .158811 .16446 
Alpha virt. eigenvalues -- 1.167551 .170461 .171371 .174601 .17981 Alpha virt. eigenvalues -- 1.183491 .186121 .191911 .196951 .20213 Alpha virt. eigenvalues -- 1.206661 .210711 .217651 .235721 .23790 Alpha virt. eigenvalues -- 1.249511 .253801 .256281 .262671 .26892 Alpha virt. eigenvalues -- 1.271981 .273951 .277911 .284231 .29220 Alpha virt. eigenvalues -- 1.300241 .304551 .306621 .310641 .31135 Alpha virt. eigenvalues -- 1.317871 .320211 .323681 .325171 .32772 Alpha virt. eigenvalues -- 1.329721 .334131 .336771 .340521 .34953 Alpha virt. eigenvalues -- 1.357261 .360051 .361341 .365561 .36802 Alpha virt. eigenvalues -- 1.372981 .386631 .389991 .407811 .41280 Alpha virt. eigenvalues -- 1.415871 .419611 .431941 .435361 .43751 Alpha virt. eigenvalues -- 1.445081 .449461 .460891 .462891 .50230 Alpha virt. eigenvalues -- 1.539131 .556951 .570081 .579751 .58597 Alpha virt. eigenvalues -- 1.590451 .596591 .597271 .599161 .60055 Alpha virt. eigenvalues -- 1.602781 .605741 .606661 .728181 .80214 Alpha virt. eigenvalues -- 1.822211 .87834

\section{Comparison of Experimental and DFT UV-Vis}

\section{Gas Phase}

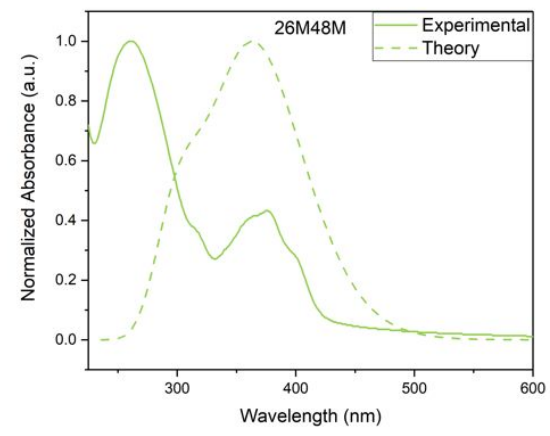

\section{Chloroform}

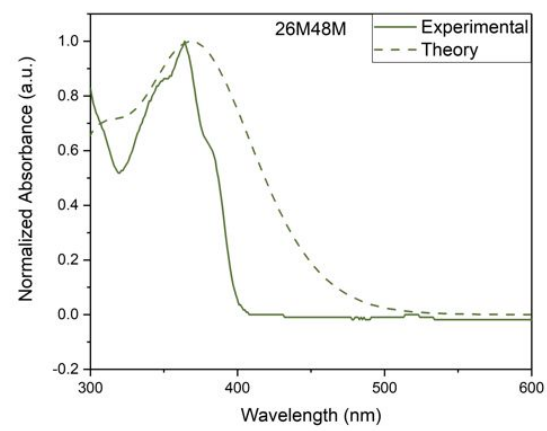

Figure S36. Gas phase and chloroform overlays of computation and experimental data for 26M48M.

\section{Lowest 15 Excited States}

\begin{tabular}{|c|c|c|c|c|c|c|}
\hline \multicolumn{4}{|c|}{ Gas Phase } & \multicolumn{3}{|c|}{ Chloroform } \\
\hline $\begin{array}{l}\text { Excited } \\
\text { state }\end{array}$ & $\frac{\text { Energy }}{(\mathrm{eV})}$ & $\frac{\text { Wavelength }}{(\mathrm{nm})}$ & $f$ & $\frac{\overline{\text { Energy }}}{(\mathrm{eV})}$ & $\frac{\text { Wavelength }}{(\mathrm{nm})}$ & $f$ \\
\hline 1 & 3.16 & 391.56 & 0.60 & 3.17 & 390.72 & 0.80 \\
\hline 2 & 3.28 & 377.85 & 0.01 & 3.28 & 377.91 & 0.01 \\
\hline 3 & 3.29 & 376.70 & 0.00 & 3.29 & 376.93 & 0.01 \\
\hline 4 & 3.49 & 356.29 & 0.75 & 3.43 & 360.84 & 0.06 \\
\hline 5 & 3.49 & 354.89 & 0.00 & 3.45 & 359.67 & 0.82 \\
\hline 6 & 3.50 & 354.06 & 0.21 & 3.46 & 358.82 & 0.14 \\
\hline 7 & 3.61 & 343.73 & 0.00 & 3.59 & 344.97 & 0.00 \\
\hline 8 & 3.79 & 326.99 & 0.00 & 3.75 & 330.52 & 0.00 \\
\hline 9 & 3.85 & 322.14 & 0.04 & 3.88 & 319.77 & 0.11 \\
\hline 10 & 3.88 & 319.40 & 0.00 & 3.92 & 315.98 & 0.00 \\
\hline 11 & 3.97 & 312.02 & 0.16 & 3.94 & 314.46 & 0.19 \\
\hline 12 & 4.11 & 302.00 & 0.59 & 4.12 & 300.66 & 0.61 \\
\hline 13 & 4.14 & 299.69 & 0.00 & 4.13 & 300.12 & 0.03 \\
\hline 14 & 4.15 & 298.66 & 0.00 & 4.14 & 299.26 & 0.16 \\
\hline 15 & 4.15 & 298.57 & 0.00 & 4.16 & 297.86 & 0.03 \\
\hline
\end{tabular}


Table S10. Gas phase and chloroform excitation energies, wavelengths, and oscillator strengths for first 15 excited state of $26 \mathrm{M} 48 \mathrm{M}$.

\section{Geometric Images}

Gas Phase
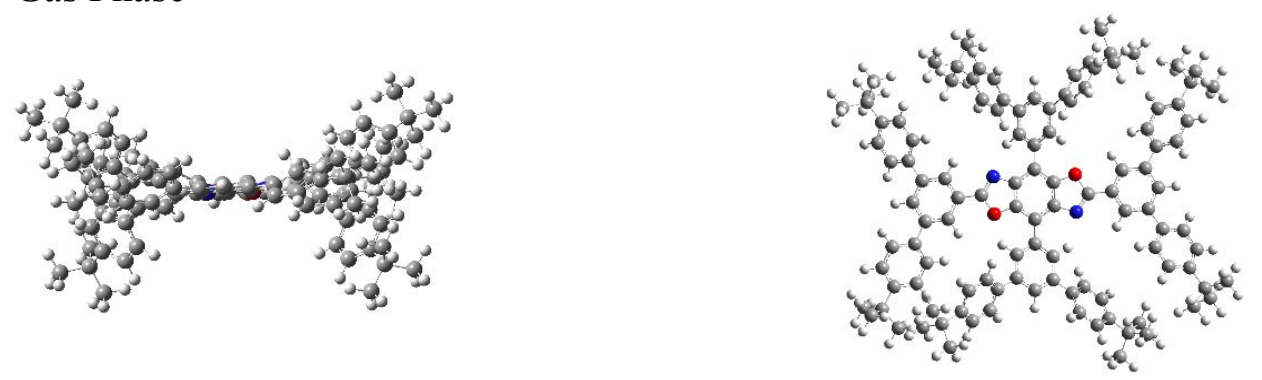

Chloroform
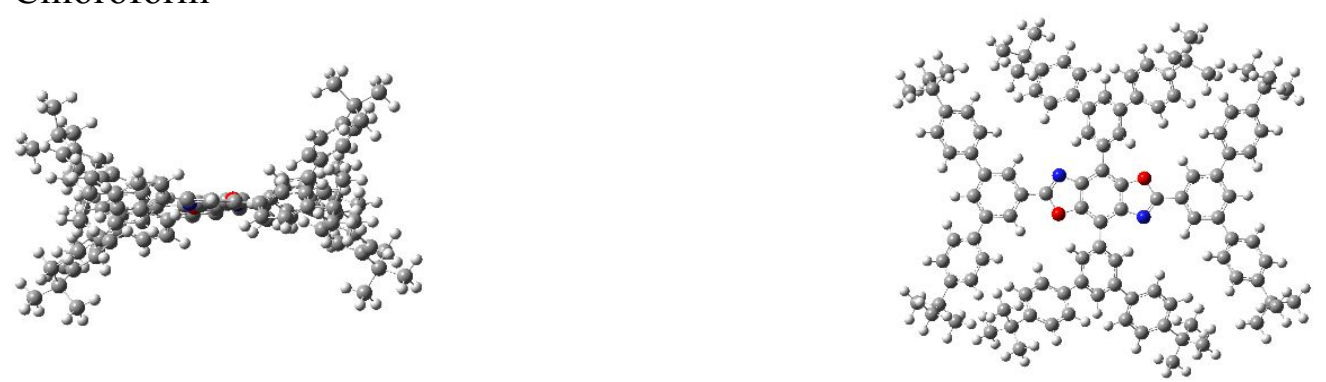

Figure S37. Geometric optimization images in gas phase and chloroform for 26M48M.

\section{DFT UV-Vis Comparisons}

\section{M, 48P, 26M48P}

Gas Phase

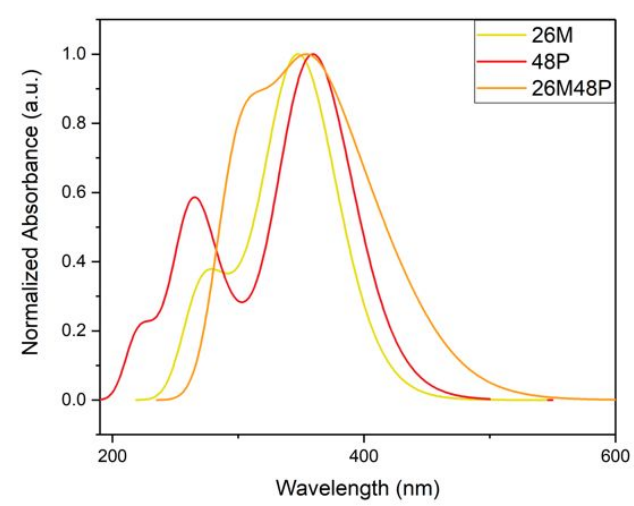

Chloroform

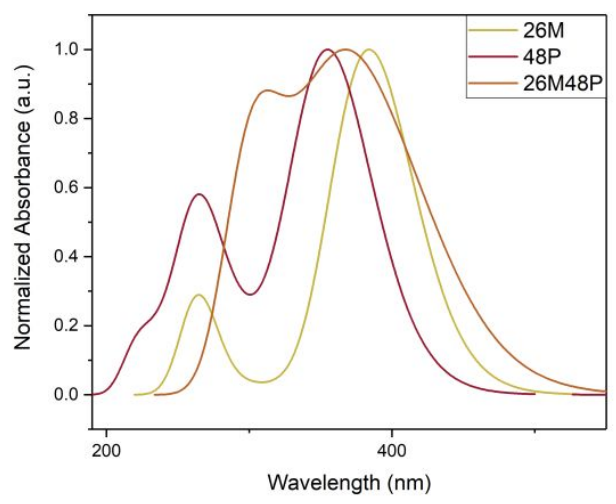

Figure S38. Theoretical UV-Vis overlays of parents 26M and 48P forming 26M48P. 
26P, 48M, 26P48M

Gas Phase

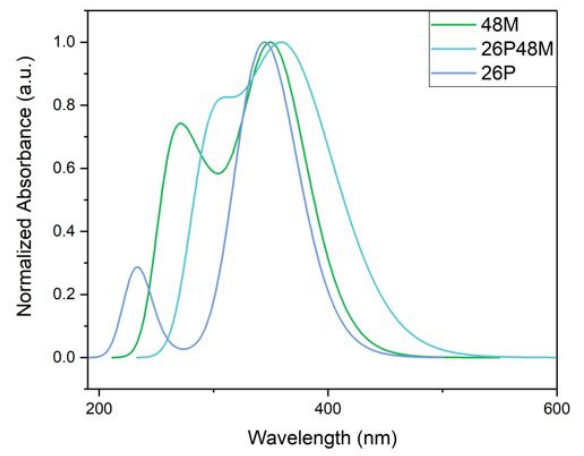

Figure S39. Theoretical UV-Vis overlays of parents 48M and 26P forming 26P48M.

26P, 48P, 26P48P

Gas Phase

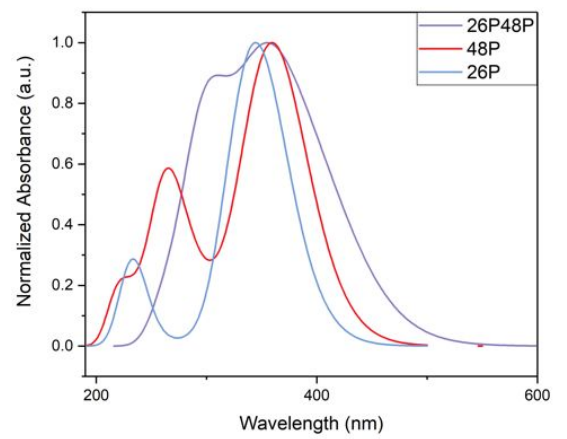

\section{Chloroform}

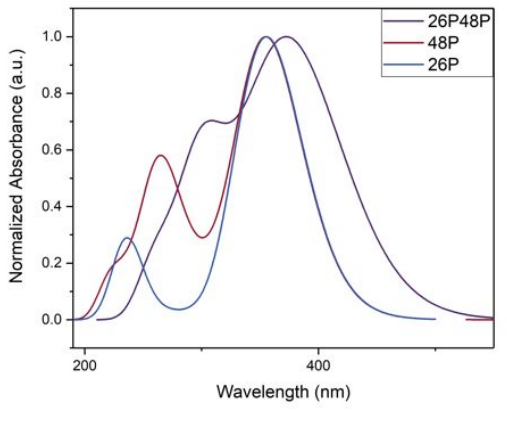

Figure S40. Theoretical UV-Vis overlays of parents 26P and 48P forming 26P48P.

\section{$26 \mathrm{M}, 48 \mathrm{M}, 26 \mathrm{M} 48 \mathrm{M}$}

Gas Phase

\section{Chloroform}
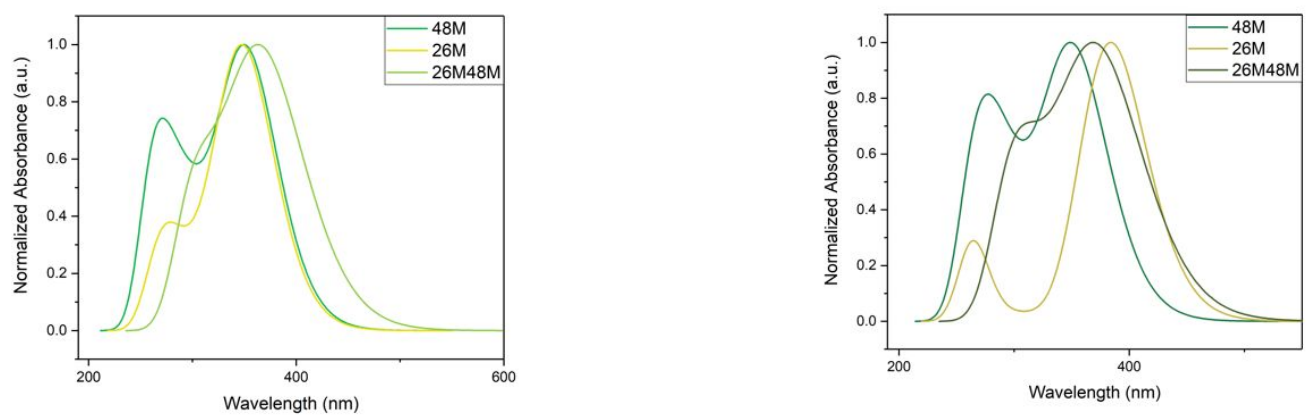

Figure S41. Theoretical UV-Vis overlays of parents 26M and 48M forming 26M48M. 
26M, 26P, 48M, 48P

Gas Phase

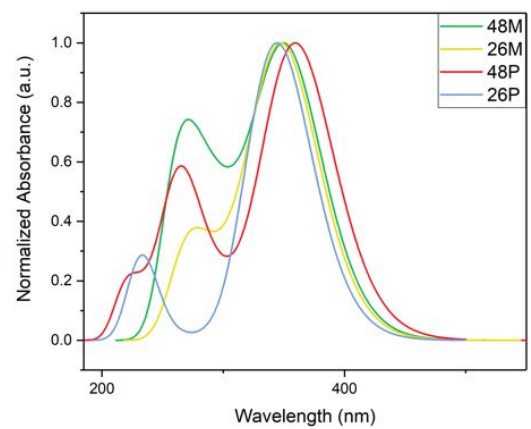

\section{Chloroform}

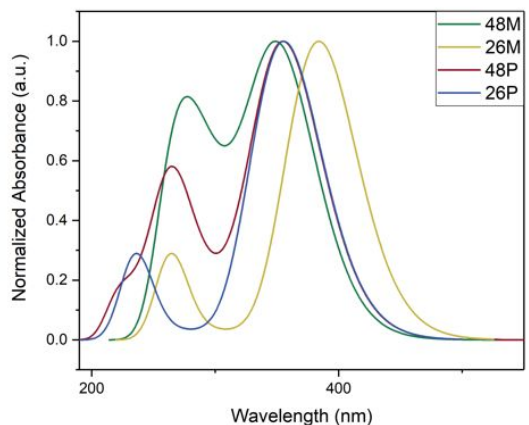

Figure S42. Theoretical UV-Vis overlays of all parent molecules in gas phase and cholorform CPCM.

26M48P, 26P48M, 26P48P, 26M48M

Gas Phase

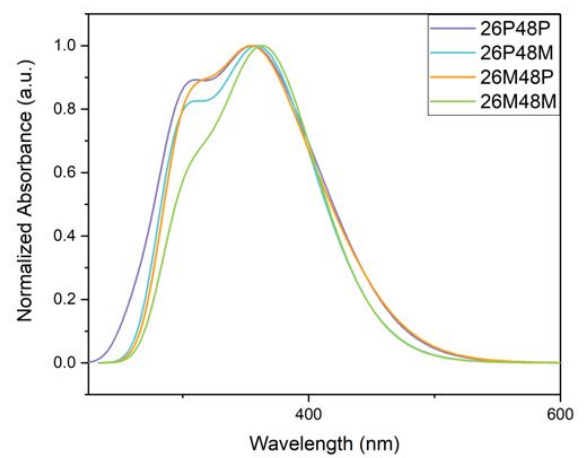

\section{Chloroform}

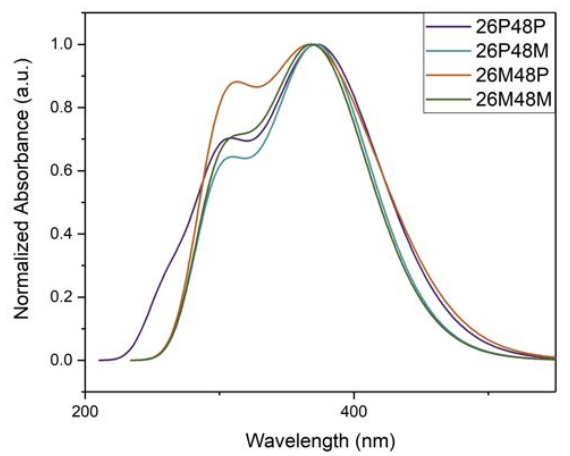

Figure S43. Theoretical UV-Vis overlays of all children molecules in gas phase and cholorform CPCM 
Frontier Molecular Orbitals
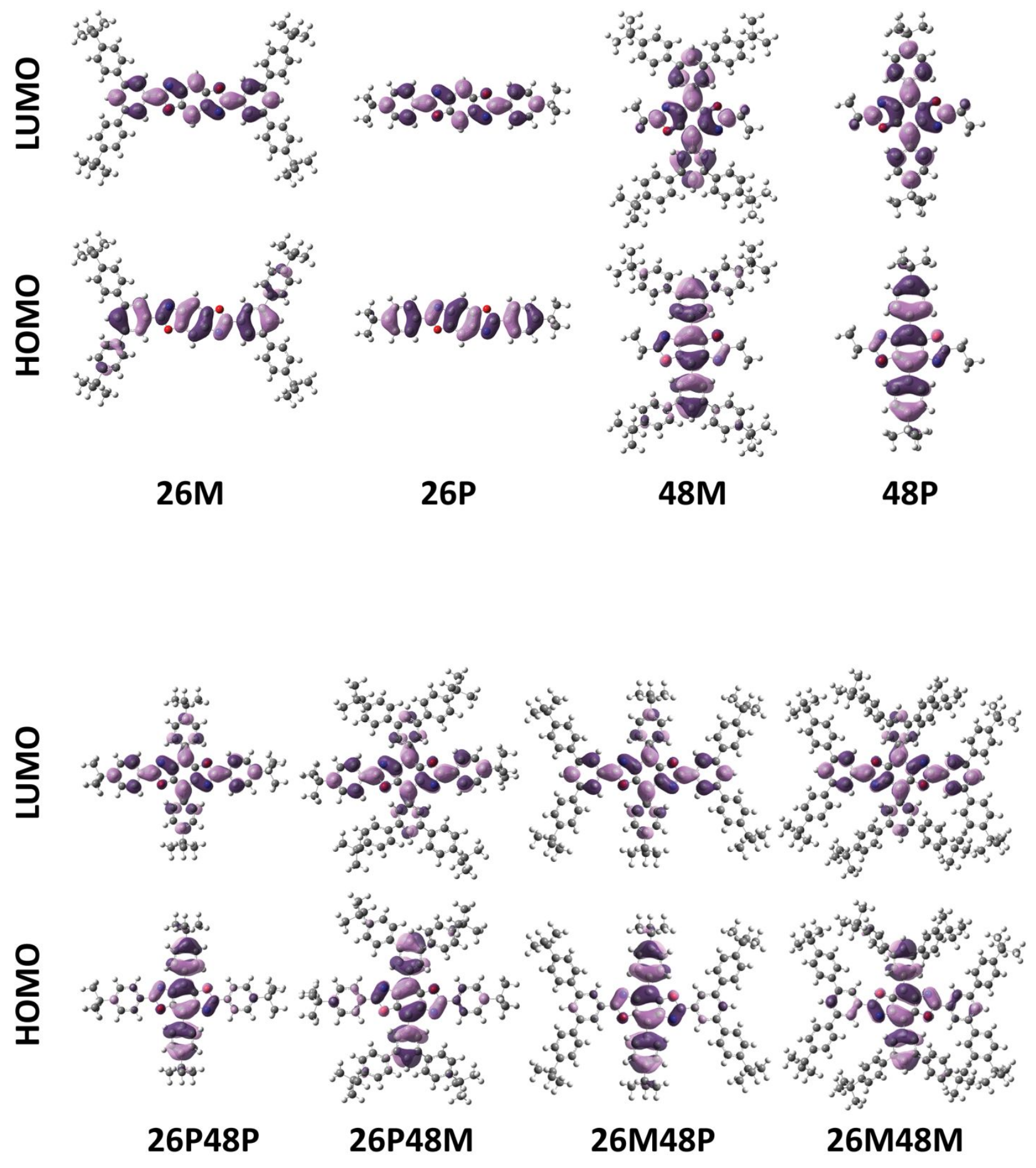

Figure S44. Frontier molecular orbitals of all investigated compounds. 